This document is downloaded from DR-NTU (https://dr.ntu.edu.sg) Nanyang Technological University, Singapore.

\title{
Micro magnetofluidic phenomena and applications
}

Zhu, Guiping

2014

https://hdl.handle.net/10356/59220

https://doi.org/10.32657/10356/59220 


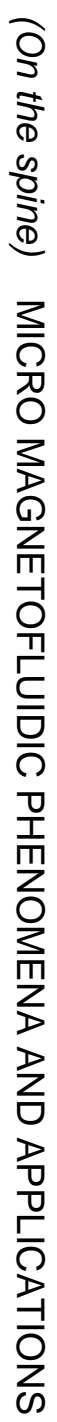
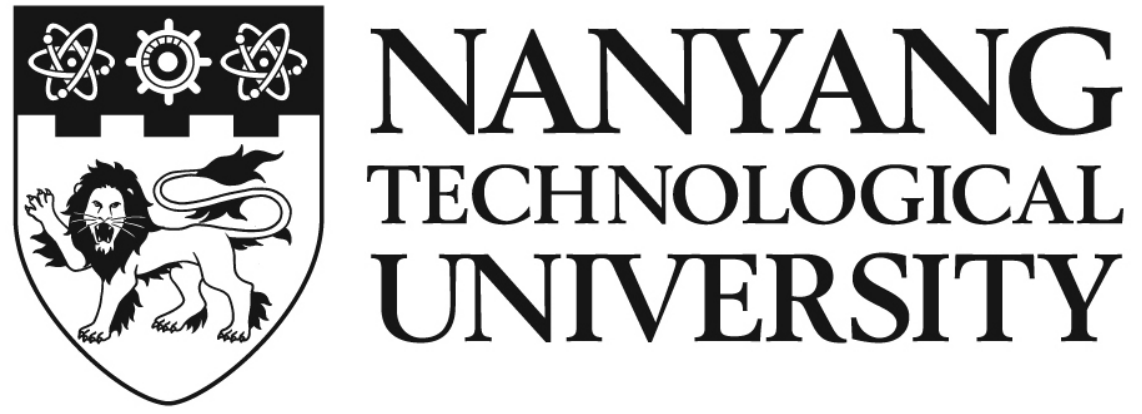

UNIVERSITY

MICRO MAGNETOFLUIDIC PHENOMENA AND APPLICATIONS

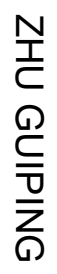

ZHU GUIPING

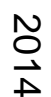

SCHOOL OF MECHANICAL \& AEROSPACE ENGINEERING 


\section{MICRO MAGNETOFLUIDIC PHENOMENA AND APPLICATIONS}

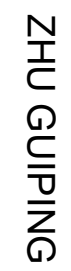

\section{ZHU GUIPING}

School of Mechanical \& Aerospace Engineering

A thesis submitted to the Nanyang Technological University

in partial fulfillment of the requirement for the degree of

Doctor of Philosophy 


\section{Acknowledgements}

First, I wish to express my deepest sense of appreciation and gratitude to my supervisor A/P Prof Huang Xiaoyang who has been abundantly helpful and offered invaluable guidance on the theoretical model development of my research work and constructive comments on my thesis writing. His diligence and devotion set us a good example to follow in our research work.

I would like to thank my previous supervisor Prof Nguyen Nam-Trung (Griffith University, Australia) for his invaluable assistance, guidance, constructive suggestions throughout the work in every step of my research. His patient encouragement and continuous support have enlightened me to a more profound and extensive academic future.

I also would like to thank my seniors Liu Jing, Che Zhizhao and Yap Yit fatt for their help on the numerical skills and critical suggestions for my research work.

I would like to take this opportunity to express my appreciation to Patrick Tan, Huang Yuli etc. for their help on my experimental setup. I would like to thank Mr. Yuan Kee Hock who gave me a lot of experimental supports. In addition, I would like to thank all the students in the researcher room 2 for their help and suggestion.

Last but not least, I would like to thank my beloved family for their encouragement and support. 


\section{Abstract}

This thesis presents studies on different applications of magnetic force in microfluidic devices, including the utilization of magnetism in digital and continuous microfluidics.

In the study on digital microfluidics, magnetism of paramagnetic solutions were used for adjusting wetting property and dynamics of liquid on a solid surface. Experiments were carried out to investigate droplet deformation under the effect of an external uniform magnetic field. The magnetic field was applied parallel to the holding surface. Droplets with different sizes were used. Droplet geometry variation was analyzed as functions of flux density and magnetization. A numerical model was developed for predicting the deformation of ferrofluid droplet undergoing a linear magnetization in a uniform magnetic field. The discrepancy between experimental and numerical results offered a qualitative estimation of droplet behaviour.

In addition, experiments were carried out with ferrofluid droplets in a permanent magnetic field. The wettability properties were investigated through contact angle variations under different magnetic field strength. The experiments were conducted using droplets with five different base diameters, and ten different magnet moving velocities ranging from $0.2 \mathrm{~mm} / \mathrm{s}$ to $2 \mathrm{~mm} / \mathrm{s}$. Two basic operating regimes, sliding and disengagement, were observed. A scaling analysis was established to describe the operation regions of the sliding droplets. The critical magnet moving velocities for droplet sliding were obtained from the scaling analysis. The investigation of magnetowetting effect and magnetic manipulation of ferrofluid droplets in particular 
or magnetic droplets in general may have potential impacts on droplet-based microfluidics interface control of liquid lenses, and possibly e-paper technology.

In the continuous microfluidics, the study was focused on investigating the interaction between a uniform magnetic field and a magnetic fluid in a microfluidic configuration. Firstly, the magnetism of paramagnetic component in microchannels was employed for spreading and transport of magnetic nanopartilcles. The experiments were carried out with two miscible fluids: mineral oil and an oil-based ferrofluid. The ferrofluid consisted of superparamagnetic nanoparticles suspended in an oil-based carrier. Under a uniform magnetic field, the superparamagnetic particles were polarized and represented magnetic dipoles. The magnetization of the magnetic nanoparticles led to a force resulting in the change of diffusion behaviour inside the microchannel. Mixing due to secondary flow close to the interface also contributed to the spreading of the ferrofluid. The magnetic force acting on the liquid/liquid interface was induced by the mismatch of magnetization between the nanoparticles and surrounding liquid in a multiphase flow system. In the investigation of magneticallyinduced spreading of magnetic nanoparticles, the stream width of ferrofluid was analyzed in terms of the applied magnetic flux intensity. The flow rate ratio was varied to test the spreading performance. In addition, a numerical simulation was carried out to verify the experimental data, taking into account the effect of particles on the flow field. The mass, momentum and diffusion equations were solved by the Finite Volume Method (FVM). These phenomena would allow simple wireless controls of a 
microfluidic system without changing the flow rates. These phenomenon can potentially be used for focusing and sorting in cytometry.

For better understanding of the magnetic nanoparticles motion under an external magnetic field, investigation was extended to ferrofluids in circular chamber with higher magnetic flux density. The flow system consisted of a water-based ferrofluid and a mixture of DI water and glycerol. The circular chamber offered a better visualization than a conventional rectangular channel due to the opaque of ferrofluid itself. The magnetic field generated a magnetic force on individual particle and thus an additional velocity. Furthermore, the mismatch in magnetization of the fluids led to instability at the interface and subsequent rapid mixing under a uniform magnetic field. The mismatch of magnetization was determined by concentration of magnetic nanoparticles. The performance of the device was analyzed based on the distribution of the magnetic nanoparticles in an interrogation window. The numerical model showed a similar trend of increasing mixing efficiency in terms of the increasing magnetic flux intensity. Almost instant mixing with an efficiency of $90 \%$ was achieved at a relatively low magnetic flux density up to $10 \mathrm{mT}$. The mixing concept demonstrated here potentially provides a wireless solution for a lab-on-a-chip system that is low-cost, robust, free of induced heat, and independent of $\mathrm{pH}$ level or ion concentration. 


\section{Table of Contents}

Acknowledgements ............................................................................................................... I

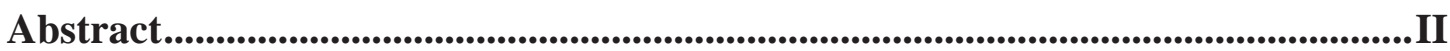

Table of Contents .....................................................................................................................V

List of Figure ...................................................................................................................... VIII

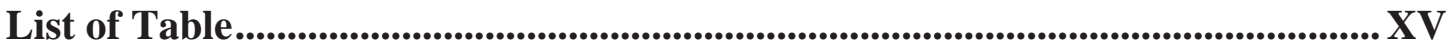

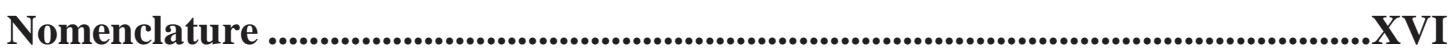

Chapter 1 Introduction.............................................................................................................1

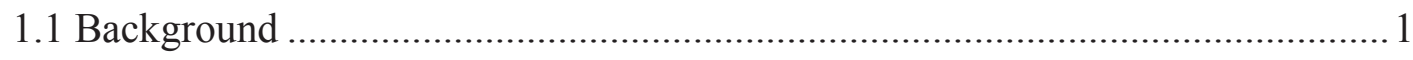

1.2 Objectives and Scopes...............................................................................

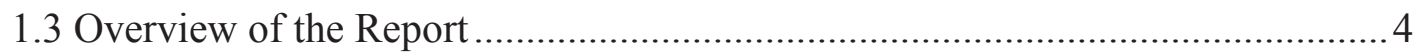

Chapter 2 Literature Review ............................................................................6

2.1 Magnetohydrodynamics (MHD) ………………………..............................

2.1.1 Governing equations of electric and magnetic fields .................................10

2.1.2 MHD-based Micropumps ............................................................................ 11

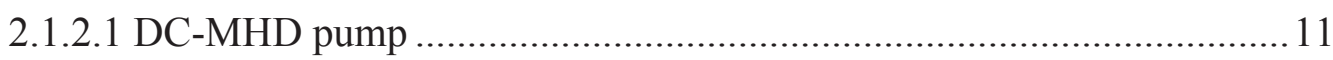

2.1.2.2 AC-MHD pump …………………………………………………..... 16

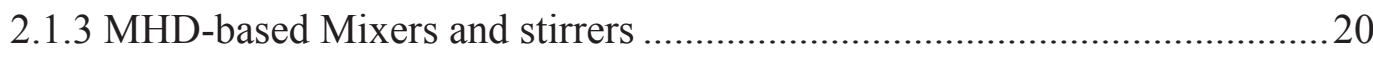

2.1.4 MHD-based pump in microfluidic network .............................................24

2.2 Magnetic particles-based applications ……………………………………......2

2.2.1 Magnetically-induced manipulation of liquid in digital microfluidics........27

2.2.1.1 Magnetic Bead Cluster Based Actuation .................................................29

2.2.1.2 Actuation of Ferrofluid Droplets .......................................................... 32

2.2.1.3 Configuration of Magnetic Field ............................................................ 34

2.2.2 Magnetically-induced manipulation of liquid in continuous microfluidics 37

2.2.2.1 Magnetophoresis (MP).......................................................................... 37

2.2.2.2 Ferrohydrodynamics (FHD).............................................................. 46 
Chapter 3 Deformation of a Ferrofluid Droplet in a Uniform Magnetic Field....56

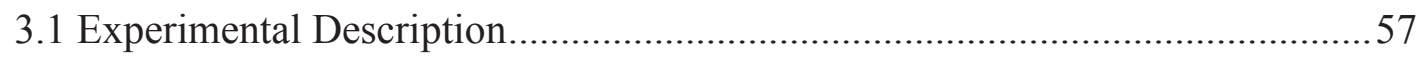

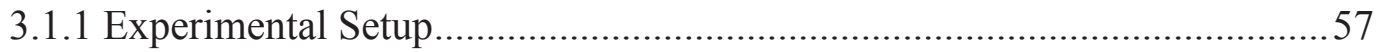

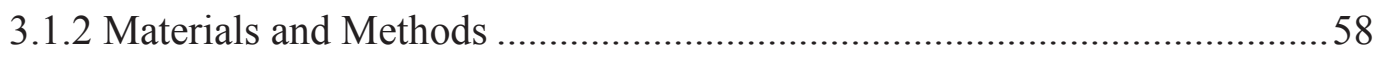

3.1.3 Stress Balance at Interface and Magnetization........................................... 61

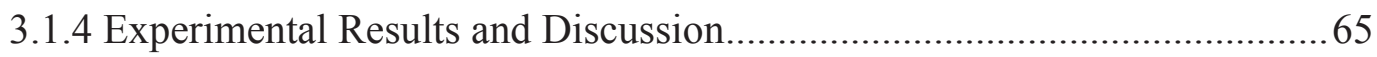

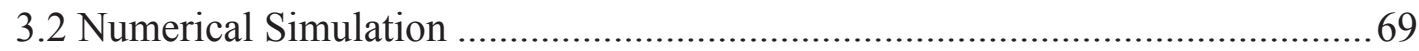

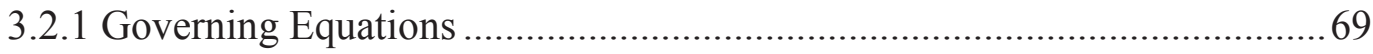

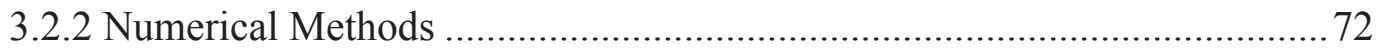

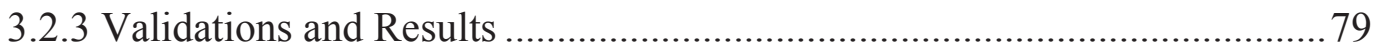

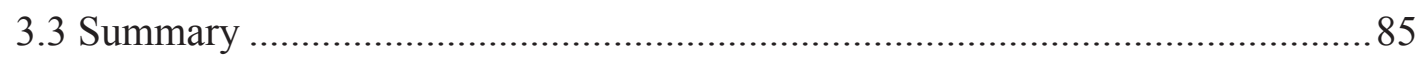

Chapter 4 Magnetowetting and Sliding Motion of a Sessile Ferrofluid Droplet .87

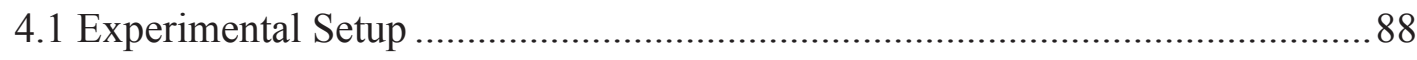

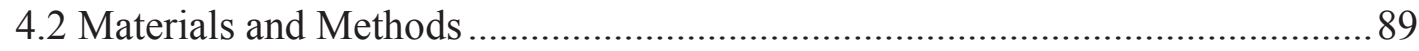

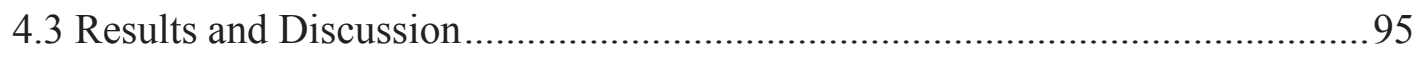

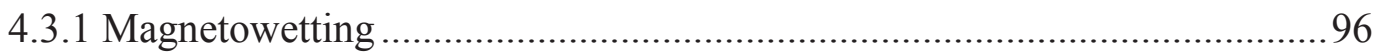

4.3.2 Sliding of a Sessile Ferrofluid Droplet (Droplet Kinematics) .................... 99

4.3.3 Scaling Analysis and Operating Map …................................................ 106

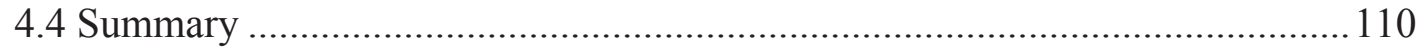

Chapter 5 Magnetofluidic Spreading in Micro-channels.....................................112

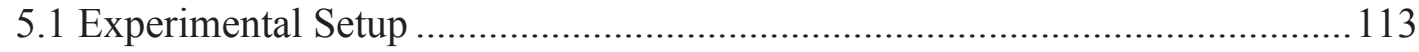

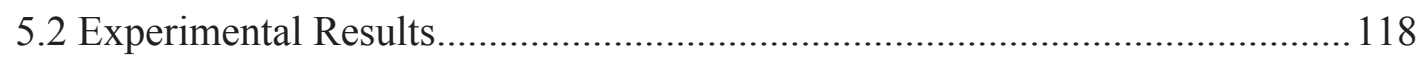

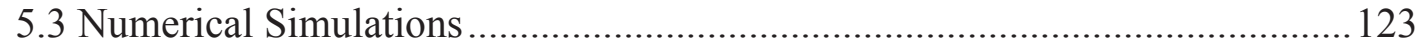

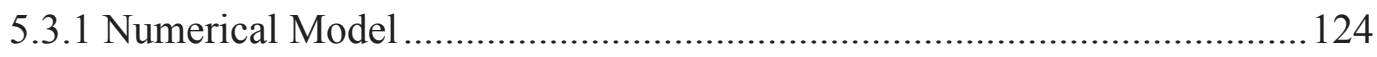

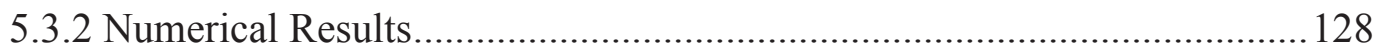

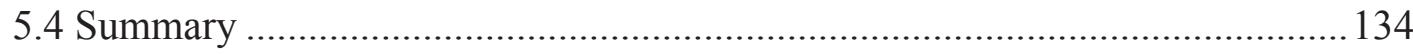

Chapter 6 Rapid magnetofluidic mixing in a uniform magnetic field ................136

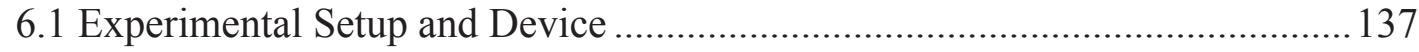

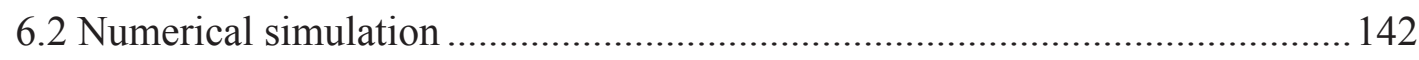

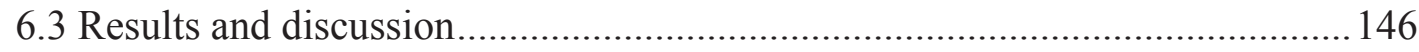




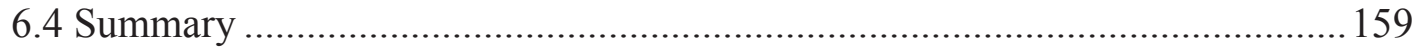

Chapter 7 Conclusions and Future Work .................................................................160

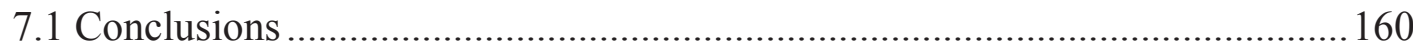

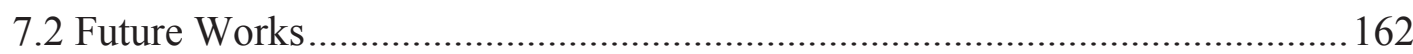

List of Publication .............................................................................................164

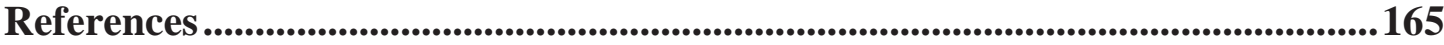




\section{List of Figure}

Figure 2-1 Inclined view of the first MHD pump [8] ................................................ 13

Figure 2-2 The channel configuration and electrodes arrangement [12] .................... 13

Figure 2-3 The illustration of operation mode of an AC type MHD micropump with diffuser/nozzle structure: (a) diffuser mode; (b) nozzle mode. [41] ............ 19

Figure 2-4 The growth of interface in an annular channel [36] ...............................20

Figure 2-5 Depiction of the pattern of the electrodes and the generated electrical

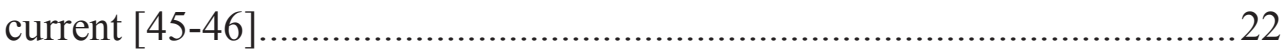

Figure 2-6 Non-intrusive MHD-stirrer (a) the arrangement of electrodes with displacement shift S; (b) flow pattern. [48]..............................................23

Figure 2-7 The branch of the microfluidic device: (a) top view; (b) side view. [49]...24

Figure 2-8 Schematic set-up and fundamental operations in a magnet-actuated droplet system [71]. 31

Figure 2-9 Device used for driving the a ferrofluid droplet [100] 35

Figure 2-10 Illustration of batchwise filter: (a) Injection of magnetic particles, (b) Trapping of magnetic particles, (c) Samples flowing, (d) Immobilization of target antigen, (e) Release of antigen. [119]. 40

Figure 2-11 Diagram of continuous magnetic separating with ferromagnetic strips fabricated inside the channel: (a) Top view of a magnetically labeled cell over a magnetic stripe; (b) experimental images of red blood cells separation from white blood cells.[124]

Figure 2-12 Schematic illustration of a magnetic SPLITT separation system. [129]..43

Figure 2-13 Schematic illustration of free-flow MP. [132]. 44

Figure 2-14 The on-chip bead separator (a) the configuration of the device; (b) the operating of the separating principle[135] 45

Figure 2-15 The typical experimental device for separation with negative magnetophoresis [140-141]. 
Figure 3-1: Experimental setup and imaging system. .57

Figure 3-2: Magnetic flux density versus applied current calibration curves of the electromagnet used in the experiments. The data points were measured using a gaussmeter.

Figure 3-3: Force balance in a multiphase system with the magnetizable liquid and the nonmagnetizable liquid (a) General condition; (b) Horizontal uniform magnetic field.

Figure 3-4 Orientational configuration for an independent particle [72] 63

Figure 3-5: Magnetization curves of the ferrofluid used in our experiments (EMG707, Ferrotec, at 285K). .64

Figure 3-6: Deformation of a sessile ferrofluid droplet under different magnetic flux densities B $(V=1.3 \mu \mathrm{L})$. 65

Figure 3-7: The volume of the droplets varies from $1.3 \mu \mathrm{L}$ to $4.8 \mu \mathrm{L}$. Experimental results of droplet parameters as a function of flux density B: (a) height; (b) width.

Figure 3-8: The volume of the droplets varies from $1.3 \mu \mathrm{L}$ to $4.8 \mu \mathrm{L}$. Experimental results of droplet parameters as a function of magnetization (with 2nd-order polynomial fit): (a) height; (b) width. 68

Figure 3-9 Grids and control volumes for two dimensional discretization. .73

Figure 3-10 The whole computational domain. 74

Figure 3-11 The interface and the seeded particles (positive particles in blue and negative particles in red): (a) Random seeding; (b) Particles after successful attraction. .77

Figure 3-12 The computational domain: (a) Uniform meshes; (b) Grid-independent study with droplet in the computational domain (solid line: $42 \times 34 \times 26 \mathrm{CVs}$ at a time step of $\Delta t=1 \times 10^{-3} \mathrm{~s}$, dashed line: $62 \times 50 \times 38 \mathrm{CVs}$ at a time step of $\left.\Delta t=5 \times 10^{-4} \mathrm{~s}\right)$. .79

Figure 3-13: Comparison between experimental and numerical results (gravity flattened effect without magnetic force): (a) experiment; (b) simulation. ...81

Figure 3-14: The magnetic potential for a sessile ferrofluid droplet in a uniform 
magnetic field. 82

Figure 3-15: The deformation of a ferrofluid droplet under different magnetic Bond numbers $(V=4.8 \mu \mathrm{L})$ : (a) experiment; (b) simulation. 83

Figure 3-16: Geometrical parameters as functions of magnetic Bond number of a 4.8$\mu \mathrm{L}$ ferrofluid droplet: (a) height; (b) width. .84

Figure 4-1 Experimental setup: (a) Stationary and sliding ferrofluid droplet; (b) Imaging system for both front view and side view of the droplet (not to scale). .88

Figure 4-2 Image projection through a single lens. .95

Figure 4-3 Sessile ferrofluid droplet (droplet III, V=2.3 $\mu \mathrm{L}$ ) under different magnetic field strengths: (a) no magnet (b) small magnet, $B=38 \pm 0.1 \mathrm{mT}$ (c) medium magnet, $B=64 \pm 0.1 \mathrm{mT}(\mathrm{d})$ large magnet, $\mathrm{B}=199 \pm 0.1 \mathrm{mT}$ 96

Figure 4-4 Magnetowetting effect of stationary ferrofluid droplets of different sizes (the lines are second-order fitting curves): (a) Base diameter (b) Droplet height (c) Apparent contact angle.

Figure 4-5 Dimensionless height varies with the magnetic Bond number. .98

Figure 4-6 Experimental procedure of a sessile ferrofluid droplet motion driven by a permanent magnet: (a) Droplet remains stationary and the magnet starts to move; (b) Pinch off moment; (c) Droplet keeps deform upon been trapped by the magnet; (d) Droplet deforms o the shape with force balance on it; (e) Sliding; (f) Disengagement. .99

Figure 4-7 Sliding of a sessile ferrofluid droplet in the presence of a moving permanent magnet $(\mathrm{h}=2 \mathrm{~mm}, \mathrm{~d}=3 \mathrm{~mm}, \mathrm{~B}=64 \mathrm{mT}, \mathrm{u}=0.2 \mathrm{~mm} / \mathrm{s}$ and $\mathrm{a} \approx 2.22$ $\mathrm{mm}$ ): (a) Evolution of the tracked droplet (every 10 frames); (b) Displacement of the droplet centroid versus time; (c) Velocity of the droplet versus time. The constant velocity of $0.2 \mathrm{~mm} / \mathrm{s}$ is plotted as the red line with a confidence band of $\pm 30 \%$; (d) Contact angles versus time. The confidence interval is $\pm 4 \%$; (e) Capillary force versus time. The confidence interval is $\pm 25 \%$. 100

Figure 4-8 Disengagement of a sessile ferrofluid droplet in the presence of a fast 
moving permanent magnet $(\mathrm{h}=2 \mathrm{~mm}, \mathrm{~d}=3 \mathrm{~mm}, \mathrm{~B}=64 \mathrm{mT}, \mathrm{u}=1.8 \mathrm{~mm} / \mathrm{s}$ and a $\approx 2.22 \mathrm{~mm}$ ): (a) Evolution of the tracked droplet (every 10 image frames); (b) Displacement of the droplet centroid versus time; (c) Velocity of the droplet versus time; (d) Contact angles versus time. The confidence interval is $\pm 4 \%$; (e) Capillary force versus time. The confidence interval is $\pm 25 \% .103$

Figure 4-9 The dependence of droplet kinematics on different parameters: (a) Magnetic strength (droplet I, magnetic velocity of $0.2 \mathrm{~mm} / \mathrm{s}$ ); (b) Base diameter of droplet (medium magnet, magnet velocity of $0.2 \mathrm{~mm} / \mathrm{s}$ ); (c) Velocity of the magnet (medium magnet, droplet II). 104

Figure 4-10 Operating map of a sliding ferrofluid droplet: (a) Scaling law for the critical velocity. (b) Operating map of the ferrofluid droplets in our experiments $\left(\beta \approx 2 \mu N s / \mathrm{mm}^{3}, \gamma \approx 8 \mu N / \mathrm{mm}\right)$. The red-shaded area is the sliding regime with the small magnet $\left(\alpha \approx 1.5 \mu \mathrm{N} / \mathrm{mm}^{3}\right)$. The green-shaded area is the sliding region of the medium magnet $\left(\alpha \approx 3 \mu \mathrm{N} / \mathrm{mm}^{3}\right)$. The sliding region of the large magnet is shaded in blue $\left(\alpha \approx 8 \mu \mathrm{N} / \mathrm{mm}^{3}\right)$. 108

Figure 5-1 Experimental setup for magnetofluidic spreading in microchannels 113

Figure 5-2 The field generation, channel and flow configuration for magnetofluidic spreading: (a) Electromagnet for generation of an uniform magnetic field; (b) Magnetofluidic spreading with ferrofluid-core configuration; Magnetofluidic spreading with ferrofluid-cladding configuration 114

Figure 5-3 The calibration of the custom-made electromagnet with flux density up to $50 \mathrm{mT}$ 115

Figure 5-4 The variation of magnetic field strength in the air gap. 116

Figure 5-5 Mass magnetization of ferrofluid (APG 2134, FerroTec) at the corresponding magnetic flux density in the air. 116

Figure 5-6 Hydrodynamic spreading in a microchannel. The flow rate of ferrofluid is fixed at $0.05 \mathrm{ml} / \mathrm{h}$ : (a) Ferrofluid-core configuration; (b) Ferrofluid-cladding configuration; (c) Overlapped interfaces of hydrodynamic spreading for ferrofluid-core configuration; (d) Overlapped interfaces of hydrodynamic spreading for ferrofluid-cladding configuration. 118 
Figure 5-7 Magnetofluidic spreading in a microchannel. The flow rates of ferrofluid and mineral oil are fixed at $0.05 \mathrm{ml} / \mathrm{h}$ and $0.2 \mathrm{ml} / \mathrm{h}$, respectively: (a) Ferrofluid-core configuration; (b) Ferrofluid-cladding configuration; (c) Overlapped interfaces of magnetofluidic spreading with ferrofluid-core configuration; (d) Overlapped interfaces of magnetofluidic spreading with ferrofluid-cladding configuration.

Figure 5-8 Magnetofludic spreading with ferrofluid-core configuration: (a) Normalized width of the core stream as functions of flux density; (b) Contour map of normalized width.

Figure 5-9 Magnetofludic spreading with ferrofluid-cladding configuration: (a) Normalized width of the core stream as functions of flux density; (b) Contour map of normalized width.

Figure 5-10 Spreading with ferrofluid-core configuration at B=2.87 $\mathrm{mT}, Q_{o} / Q_{F F}=4$ : (a) $\mathrm{x}-\mathrm{y}$ plane; (b) y-z plane. 128

Figure 5-11 Spreading with ferrofluid-cladding configuration at $\mathrm{B}=2.87 \mathrm{mT}, Q_{\delta} / Q_{F F}=4$ : (a) x-y plane; (b) y-z plane.

Figure 5-12 Depth averaged concentration across the channel width at different field strengths $\left(Q_{o} / Q_{F F}=4\right):$ (a) Magnetofluidic spreading with ferrofluid-core configuration; (b) Magnetofluidic spreading with ferrofluid-cladding configuration.

Figure 5-13 Comparison between experimental and numerical results with $Q_{o} / Q_{F F}=4$ :

(a) Magnetofluidic spreading with ferrofluid-core configuration; (b) Magnetofluidic spreading with ferrofluid-cladding configuration

Figure 6-1 Magnetofluidic mixing in a uniform magnetic field: (a) Fabricated microfluidic device; (b) Custom-made electromagnet; (c) Ferrofluid-core configuration; (d) Ferrofluid-cladding configuration. 137

Figure 6-2 Fabrication of microchannels with soft lithography: (a) SU-8 spin coated on a silicon wafer; (b) UV exposure with a mask; (c) development of SU-8 mold; (d) pouring PDMS on the mold; (e) peeling off the PDMS; (f) surface treatment and bond; $(\mathrm{g})$ the fabricated microchannel. 139 
Figure 6-3 Mixing efficiency as a function of magnetic flux density for ferrofluid-core configuration, the viscosity of ferrofluid and water/glycerol mixture are both fixed at $5 \mathrm{mPa} \cdot \mathrm{s}$ : (a) The flow rate of ferrofluid is fixed at $0.5 \mathrm{ml} / \mathrm{h}$ while the flow rate of mixture varies from $0.25 \mathrm{ml} / \mathrm{h}$ to $0.75 \mathrm{ml} / \mathrm{h}$; (b) The flow rates of ferrofluid and mixture vary from $0.25 \mathrm{ml} / \mathrm{h}$ to $0.75 \mathrm{ml} / \mathrm{h}$, but maintain a fixed ratio of 1 . 148

Figure 6-4 Mixing efficiency as a function of magnetic flux density for ferrofluidcladding configuration, the viscosity of ferrofluid and mixture are fixed at 5 $\mathrm{mPa} \cdot \mathrm{s}$ and $5 \mathrm{mPa} \cdot \mathrm{s}$ respectively. (a) The flow rate of ferrofluid is fixed at 0.5 $\mathrm{ml} / \mathrm{h}$ while the flow rate of mixture varies from $0.25 \mathrm{ml} / \mathrm{h}$ to $0.75 \mathrm{ml} / \mathrm{h}$; (b) The flow rates of ferrofluid and mixture vary from $0.25 \mathrm{ml} / \mathrm{h}$ to $0.75 \mathrm{ml} / \mathrm{h}$, but maintain at a fixed ratio of 1 . 149

Figure 6-5 Mixing efficiency as a function of magnetic flux density for the same flow rate ratio of 1: (a) Ferrofluid-cladding configuration with viscosity of ferrofluid fixed at $5 \mathrm{mPa} \cdot \mathrm{s}$ while the viscosity of mixture varies from 2.5 $\mathrm{mPa} \cdot \mathrm{s}$ to $7.5 \mathrm{mPa} \cdot \mathrm{s}$; (b) Ferrofluid-core configuration with viscosity of ferrofluid fixed at $5 \mathrm{mPa} \cdot \mathrm{s}$ while the viscosity of mixture varies from 2.5 $\mathrm{mPa} \cdot \mathrm{s}$ to $7.5 \mathrm{mPa} \cdot \mathrm{s}$. 150

Figure 6-6 Simulated velocity and concentration fields of ferrofluid-core configuration:

(a) no magnetic field; (b) with magnetic field 153

Figure 6-7 Simulated velocity and concentration fields of ferrofluid-cladding configuration: (a) no magnetic field; (b) with magnetic field. 153

Figure 6-8 Numerically simulated mixing efficiency at the different magnetic flux densities for both the ferrofluid-core and ferrofluid-cladding configurations.

Figure 6-9 Mixing of a three-fluids system consisting of fluorescent diamagnetic fluid, ferrofluid and non-fluorescent diamagnetic fluid: (a) Intensity profile across the mixing chamber in a bright field image with epi-fluorescent filter; (b) Intensity profile in a fluorescent image with epi-fluorescent filter only. ... 156

Figure 6-10 Intensity profile across the mixing chamber with a two-fluids system 
consisting of a ferrofluid and a fluorescent diamagnetic fluid. 158 


\section{List of Table}

Table 3-1 Volume and contact angle of droplets in calibration process......................59

Table 4-1 Droplets with different sizes under investigation. ..................................... 91

Table 4-2 Frame rates used for the different speeds of the magnets........................... 91

Table 6-1 Different water/glycerol mixture. ........................................................... 140 


\section{Nomenclature}

\begin{tabular}{|c|c|}
\hline$\vec{M}$ & Magnetization $[\mathrm{A} / \mathrm{m}]$ \\
\hline$\vec{m}$ & Magnetic moment $\left[\mathrm{A} \mathrm{m}^{2}\right]$ \\
\hline$n_{m}$ & Number density of magnetic moments \\
\hline$\vec{H}$ & Magnetic field strength $[\mathrm{A} / \mathrm{m}]$ \\
\hline$\vec{B}$ & Magnetic flux density [T] \\
\hline$\vec{P}$ & Polarization $\left[\mathrm{C} / \mathrm{m}^{2}\right]$ \\
\hline$\vec{D}$ & Electric displacement field $\left[\mathrm{C} / \mathrm{m}^{2}\right]$ \\
\hline$\vec{F}_{m p}$ & Magnetic force per unit volume exerted on the bead cluster $\left[\mathrm{N} / \mathrm{m}^{3}\right]$ \\
\hline$\vec{F}_{m p x}$ & Horizontal component of $\vec{F}_{m p}\left[\mathrm{~N} / \mathrm{m}^{3}\right]$ \\
\hline$\vec{M}_{x}$ & $\mathrm{X}$ component of magnetization $[\mathrm{A} / \mathrm{m}]$ \\
\hline$\vec{M}_{y}$ & Y component of magnetization $[\mathrm{A} / \mathrm{m}]$ \\
\hline$\vec{M}_{z}$ & $\mathrm{Z}$ component of magnetization $[\mathrm{A} / \mathrm{m}]$ \\
\hline$\vec{H}_{x}$ & $\mathrm{X}$ component of magnetic field strength $[\mathrm{A} / \mathrm{m}]$ \\
\hline$\vec{F}_{m}$ & Volumetric magnetic force $[\mathrm{N}]$ \\
\hline$\vec{F}_{g}$ & Gravitational force $[\mathrm{N}]$ \\
\hline$\vec{F}_{\sigma}$ & Interfacial force $[\mathrm{N}]$ \\
\hline$\vec{F}_{\text {mag }}$ & Magnetic force on a single particle $[\mathrm{N}]$ \\
\hline$m_{b}$ & Mass of the bead cluster $[\mathrm{kg}]$ \\
\hline$\vec{F}_{f}$ & Friction force $[\mathrm{N}]$ \\
\hline
\end{tabular}




\begin{tabular}{|c|c|}
\hline$K_{f}$ & Friction constant \\
\hline$r_{c}$ & Radius of contact area at the bottom of the droplet $\mathrm{V}$ velocity $[\mathrm{m}]$ \\
\hline$\vec{u}$ & Fluid velocity $[\mathrm{m} / \mathrm{s}]$ \\
\hline$L_{c}$ & Length of the outer circumference of the sphere $[\mathrm{m}]$ \\
\hline$\vec{F}_{c a p}$ & Capillary force $[\mathrm{N}]$ \\
\hline$\vec{M}_{s}$ & Saturation moment of the ferrofluid $\left[\mathrm{A} \mathrm{m}^{2}\right]$ \\
\hline$\vec{M}_{d}$ & Saturation moment of the magnetic solid in the bulk $\left[\mathrm{A} \mathrm{m}^{2}\right]$ \\
\hline$k_{B}$ & Boltzmann factor $[\mathrm{J} / \mathrm{K}]$ \\
\hline$T$ & Absolute temperature in applied field $[\mathrm{K}]$ \\
\hline$t$ & Time $[s]$ \\
\hline$\vec{J}$ & Electric current density $\left[\mathrm{A} / \mathrm{m}^{2}\right]$ \\
\hline$\vec{E}$ & Electric field strength $[\mathrm{N} / \mathrm{C}]$ \\
\hline $\operatorname{Re}_{m}$ & Magnetic Reynolds number \\
\hline$N$ & Stuart number \\
\hline$H a$ & Hartmann number \\
\hline$h$ & Channel height $[\mathrm{m}]$ \\
\hline$w$ & Channel width [m] \\
\hline$l$ & Channel length [m] \\
\hline$d$ & Diameter $[\mathrm{m}]$ \\
\hline$\vec{F}_{L}$ & Lorentz force $[\mathrm{N}]$ \\
\hline$Q$ & Volumetric flow rate $\left[\mathrm{m}^{3} / \mathrm{s}\right]$ \\
\hline
\end{tabular}




\begin{tabular}{|c|c|}
\hline$V_{p}$ & Volume of particle $\left[\mathrm{m}^{3}\right]$ \\
\hline$\vec{F}_{d}$ & Stokes' drag force $[\mathrm{N}]$ \\
\hline$d_{p}$ & Particle diameter $[\mathrm{m}]$ \\
\hline$r_{p}$ & Particles radius $[\mathrm{m}]$ \\
\hline$p^{*}$ & Composite pressure $[\mathrm{Pa}]$ \\
\hline$z$ & Elevation of the point above a reference plane [m] \\
\hline$p_{m}$ & Fluid-magnetic pressure $[\mathrm{Pa}]$ \\
\hline$p_{n}$ & Magnetic normal pressure $[\mathrm{Pa}]$ \\
\hline$p_{0}$ & Pressure in the nonmagnetic fluid $[\mathrm{Pa}]$ \\
\hline$p_{c}$ & Capillary pressure $[\mathrm{Pa}]$ \\
\hline$p$ & Thermodynamic pressure $[\mathrm{Pa}]$ \\
\hline$H$ & Magnitude of magnetic field strength $[\mathrm{A} / \mathrm{m}]$ \\
\hline$M$ & Magnitude of magnetization $[\mathrm{A} / \mathrm{m}]$ \\
\hline$\vec{M}_{n}$ & Magnitude of magnetization in normal direction $[\mathrm{A} / \mathrm{m}]$ \\
\hline$R$ & Radius of curvature \\
\hline$t_{n n}$ & Magnetic surface force density $\left[\mathrm{N} / \mathrm{m}^{3}\right]$ \\
\hline$M_{s}$ & Saturation magnetization $[\mathrm{A} / \mathrm{m}]$ \\
\hline$s$ & Mass magnetization $\left[\mathrm{Am}^{2} / \mathrm{kg}\right]$ \\
\hline$H(\phi)$ & Smoothed heaviside function \\
\hline$P$ & Pressure $[\mathrm{Pa}]$ \\
\hline$B_{m}$ & Magnetic Bond number \\
\hline
\end{tabular}




\begin{tabular}{|c|c|}
\hline$V$ & Volume of droplet $\left[\mathrm{m}^{3}\right]$ \\
\hline$A$ & Contact area \\
\hline$H_{0}$ & Initial magnetic field strength $[\mathrm{A} / \mathrm{m}]$ \\
\hline Bo & Gravitational Bond number \\
\hline$B o_{c r}$ & Critical gravitational Bond number \\
\hline$r_{c r}$ & Critical radius of droplet $[\mathrm{m}]$ \\
\hline$a$ & Base diameter of droplet $[\mathrm{m}]$ \\
\hline$b$ & Height of droplet $[\mathrm{m}]$ \\
\hline$u_{c r}$ & Critical velocity $[\mathrm{m} / \mathrm{s}]$ \\
\hline$D_{f}$ & Diffusion coefficient $\left[\mathrm{m}^{2} / \mathrm{s}\right]$ \\
\hline$D_{h}$ & Hydraulic channel diameter $[\mathrm{m}]$ \\
\hline$U$ & Average velocity in the channel $[\mathrm{m} / \mathrm{s}]$ \\
\hline$C$ & Non-dimensional volume concentration \\
\hline$\vec{u}_{p}$ & Velocity of the magnetic particles $[\mathrm{m} / \mathrm{s}]$ \\
\hline$C_{V}$ & Volume concentration \\
\hline$Q_{F F}$ & Flow rate of ferrofluid $\left[\mathrm{m}^{3} / \mathrm{s}\right]$ \\
\hline$Q_{o}$ & Flow rate of oil $\left[\mathrm{m}^{3} / \mathrm{s}\right]$ \\
\hline$\vec{J}_{f}$ & Free current density $[\mathrm{A} / \mathrm{m} 2]$ \\
\hline$S$ & Source term \\
\hline$\vec{x}_{p}$ & Initial location of the particle $[\mathrm{m}]$ \\
\hline$\vec{x}_{n e w}$ & New position of the particle $[\mathrm{m}]$ \\
\hline
\end{tabular}




$\begin{array}{ll}\vec{N}\left(\vec{x}_{p}\right) & \text { Unit normal vector at particle location } \\ \vec{u}\left(\vec{x}_{p}\right) & \text { Velocity of particle }[\mathrm{m} / \mathrm{s}] \\ S_{d} & \text { Blur spot diameter [m] } \\ f_{x} & \text { Optical focal length [m] } \\ c & \text { Lens f-number } \\ N_{f} & \text { Distance from the focal plane to the lens [m] } \\ x_{S} & \text { Distance between the subject and the focus plane [m] }\end{array}$

\section{Greek symbols}

\begin{tabular}{|c|c|}
\hline$\mu_{0}$ & Permeability of free space $\left[\mathrm{N} / \mathrm{A}^{2}\right]$ \\
\hline$\varepsilon_{0}$ & Electric constant indicates the permittivity of free space $[\mathrm{F} / \mathrm{m}]$ \\
\hline$\chi_{e}$ & Electric susceptibility \\
\hline$\rho_{f}$ & Free charge density $\left[\mathrm{C} / \mathrm{m}^{3}\right]$ \\
\hline$\sigma$ & Surface tension $[\mathrm{N} / \mathrm{m}]$ \\
\hline$\rho_{b}$ & Density of bead cluster $\left[\mathrm{kg} / \mathrm{m}^{3}\right]$ \\
\hline$\vec{\sigma}_{s w}$ & Interfacial tension between silicone oil and water $[\mathrm{N} / \mathrm{m}]$ \\
\hline$\phi_{\mathrm{vol}}$ & Volume fraction \\
\hline$\xi$ & Ratio between magnetic moment energy and the thermal energy \\
\hline$\kappa$ & Electrical conductivity $\left[\mathrm{A}^{2} \mathrm{~s}^{3} \mathrm{~m}^{-3} \mathrm{~kg}^{-1}\right]$ \\
\hline$\lambda$ & Magnetic diffusivity $\left[\mathrm{N}^{-1} \mathrm{~s}^{-3} \mathrm{~m}^{3} \mathrm{~kg}\right]$ \\
\hline$\rho$ & Density of the material $\left[\mathrm{kg} / \mathrm{m}^{3}\right]$ \\
\hline
\end{tabular}


$\Delta \rho \quad$ Density difference between two fluids $\left[\mathrm{kg} / \mathrm{m}^{3}\right]$

$\eta \quad$ Liquid viscosity $[\mathrm{Pa} \cdot \mathrm{s}]$

$\omega \quad$ Angular frequency $[\mathrm{rad} / \mathrm{s}]$

$\varphi \quad$ Phase angle [degree]

$\chi_{p} \quad$ Susceptibility of particle

$\chi_{f} \quad$ Susceptibility of fluid

$\zeta \quad$ Magnetophoretic mobility $\left[\mathrm{m}^{2} \mathrm{~Pa}^{-1} \cdot \mathrm{s}^{-1}\right]$

$\chi_{m} \quad$ Susceptibility of magnetic fluid

$\chi_{0} \quad$ Initial value of magnetic susceptibility

$\psi \quad$ Magnetic scalar potential $\left[\mathrm{V} \cdot \mathrm{s} \cdot \mathrm{m}^{-1}\right]$

$\theta_{m} \quad$ Angle between dipole moment and field direction [degree]

$\eta_{F F 0} \quad$ Zero-shear viscosity of ferrofluid $[\mathrm{Pa} \cdot \mathrm{s}]$

$\eta_{F F} \quad$ Viscosity of ferrofluid $[\mathrm{Pa} \cdot \mathrm{s}]$

$\rho_{F F} \quad$ Density of ferrofluid $\left[\mathrm{kg} / \mathrm{m}^{3}\right]$

$\eta_{o} \quad$ Viscosity of oil [Pa·s]

$\rho_{o} \quad$ Density of oil $\left[\mathrm{kg} / \mathrm{m}^{3}\right]$

$\theta \quad$ Static contact angle [degree]

$\ell \quad$ Capillary length $[\mathrm{m}]$

$\theta_{a} \quad$ Advancing contact angle [degree]

$\theta_{r} \quad$ Receding contact angle [degree]

$\alpha \quad$ Factor represents the strength of the magnetic field $\left[\mu \mathrm{N} / \mathrm{mm}^{3}\right]$ 


\begin{tabular}{|c|c|}
\hline$\beta$ & Factor represents the friction $\left[\mu \mathrm{Ns} / \mathrm{mm}^{3}\right]$ \\
\hline$\gamma$ & Factor represents the strength of capillary $[\mu \mathrm{N} / \mathrm{mm}]$ \\
\hline$\Delta v$ & Error of the evaluated velocity \\
\hline$\delta_{x}$ & Displacement of the centroid of the droplet between two frames [m] \\
\hline$\delta_{t}$ & Time delay between two frames $[\mathrm{s}]$ \\
\hline$\Delta \delta_{x}$ & Systematic error caused by camera resolution \\
\hline$\Delta \delta_{t}$ & Systematic error caused by the timer of the frame grabber \\
\hline$\varepsilon$ & Relative systematic error \\
\hline$\rho_{G}$ & Density of glycerol $\left[\mathrm{kg} / \mathrm{m}^{3}\right]$ \\
\hline$\eta_{G}$ & Viscosity of glycerol $[\mathrm{Pa} \cdot \mathrm{s}]$ \\
\hline$\rho_{\operatorname{mix}}$ & Density of water/glycerol mixture $\left[\mathrm{kg} / \mathrm{m}^{3}\right]$ \\
\hline$\eta_{m i x}$ & Viscosity of water/glycerol mixture $[\mathrm{Pa} \cdot \mathrm{s}]$ \\
\hline$\vartheta$ & Mixing efficiency \\
\hline$\Phi$ & Dependent variable \\
\hline$\phi(\vec{x}, t)$ & Signed normal distance function \\
\hline$\Gamma$ & Interface \\
\hline
\end{tabular}

\section{Acronyms}

2D

Two-dimensional

3D

Three-dimensional

BPB

Bromophenol blue

CFD

Computational fluid dynamics 
MM Magnetophoretic mobility

CTV Cell tracking velocimetry

FFF Free flow fractionation

SPLITT Split flow thin fractionation

MP Magnetophoresis

MHD Magnetohydrodynamics

FHD Ferrohydrodynamics

MR Magnetorheology

FFM Free-flow magnetophoresis

SWCNTs Single-walled carbon nanotubes

FVM Finite volume method

FDM Finite difference method

LIGA Lithographie Galvanoformung, Abformung

NdFeB Neodymium-iron-boron

NMR Nuclear magnetic resonance

LTCC Low temperature co-fired ceramic tapes

FEM Finite element method

PCR Polymerase chain reaction

PMMA Polymethyl methacrylate

MRI Magnetic resonance imaging

MACS Magnetic activated cell sorting 
PBLs Peripheral blood leukocytes

$\mathrm{Ni} / \mathrm{Fe} \quad$ Nickel-iron

Gd-DTPA Diethylenetriamine-pentaacetic acid

CF Continuous-flow

WENO Weighted essentially non-oscillatory

TVD Total variation diminishing

PDMS Polydimethylsiloxane

LLF Local Lax-Friedrichs

PLS Particle level-set

DOF Depth of filed 


\section{CHAPTER 1}

\section{INTRODUCTION}

\subsection{Background}

The development of microfluidics was triggered by the use of silicon-based micromachining technology for fluidic applications such as ink jet printing in the 1980s. Today, biotechnological applications inspired the development of portable microfluidic device with low cost and high sensitivity. Microfluidic systems, that involve flow in microscale have brought remarkable progress to biotechnology. Droplet-based microfluidics and digital microfluidics has attracted considerable attention because of advantages such as lower cost, low sample consumption, higher flexibility etc.

The manipulation in microfluidic devices can be achieved by both passive and active ways. The passive ways rely purely on the configuration and property of the liquids to be manipulated. The active realization requires external fields such as pressure, temperature, electric and acoustic fields. Most active concepts require a complex design for inducing the external field. In some of the active applications utilizing electric and optical concepts, the generation of unnecessary heat will be harmful for sensitive samples. The application of magnetism in microfluidics aims at overcoming the above-mentioned problem and gains new functions of the microfluidic devices. The interaction between magnetism and fluid flow provides a wireless approach for microfluidic device not affected by the heat generation, $\mathrm{pH}$ level or ion 
concentration. In most cases, the application of magnetic force does not suffer from the geometric restrictions.

In digital microfluidics, the manipulation of small amount magnetic liquid on a solid surface can be achieved by inducing magnetic force. The wetting property as well as the dynamic behaviour are tunable in terms of the external magnetic flux density. In continuous microfluidics, magnetic application can be employed for conducting liquid driven by Lorentz force. Furthermore, magnetic particles are commonly introduced for inducing magnetic component in a diamagnetic carrier. The types of magnetic fluids results in the research areas such as ferrohydrodynamics (FHD), magnetorheology (MR), and magnetophoresis (MP). For applications involving ferrofluid, the transport of magnetic nanoparticles is achieved by magnetic force on individual particles and volumetric magnetic volume force on fluid flow. In a diluted ferrofluid, the manipulation of larger particles is realized with negativemagnetophoresis principle. In magnetorheologyical fluids, the chain formation of large particles is utilized for adjusting liquid viscosity. In magnetophoresis, magnetic particles need to be considered as discrete items. Magnetic force on discrete magnetic particles enables the separation process in a microfluidic channel.

\subsection{Objectives and Scopes}

The main objective of this research is focused on development and investigation of micro magnetofluidic devices. In particular, the study looks into the details of ferrofluid droplets in microchannels driven by a magnetic field and the mixing of 
ferrofluids in microchannels.

Following are the scopes of this study:

(1) Carrying out experiments to investigate wetting properties of sessile ferrofluid droplets on a hydrophobic solid surface placed in mineral oil. Designing and fabricating a sample container for handling droplets. Applying a uniform magnetic field generated by an electromagnet parallel to the solid surface.

(2) Using a three-dimensional (3D) numerical model to calculate the droplet behaviour under the effect of the applied magnetic field. Discussing the effect and comparing the results with the experimental data.

(3) Using a permanent magnetic field to investigate both wetting properties and the motion of a sessile ferrofluid droplet. Using a camera setup to record droplets with volume ranging from 0.7 to $4.8 \mu \mathrm{L}$.

(4) Conducting a theoretical analysis to study the motion of ferrofluid droplets by considering the force balance.

(5) Making an electromagnet from a transformer and get the calibration data of the electromagnetic field by a commercial Gaussmeter. Carrying out the experimental testing for spreading of ferrofluid stream with various magnetic flux densities. Using different flow configuration for the testing: ferrofluid-core and ferrofluidcladding configuration. Proceeding with the results analysis by measuring the width of ferrofluid stream by a MATLAB program.

(6) Implementing a 3D model to obtain the numerical results for ferrofluid stream width varies with different magnetic flux density. Comparing the numerical and 
experimental results.

(7) Using the same electromagnet in the spreading experiment to carry out the experiment on mixing in a circular chamber. Applying different flow configuration for the testing: ferrofluid-core and ferrofluid-cladding configuration. Adjusting the viscosity and flow rate ratio between the magnetic and diamagnetic fluids to achieve different initial condition and mixing behaviour.

(8) Implementing a two-dimensional (2D) model to obtain the numerical results for mixing efficiency of magnetic nanoparticles varies with different magnetic flux density. Comparing the numerical and experimental results.

\subsection{Overview of the Report}

Chapter 1 gives a general introduction to the background, objectives and scopes of research on micro magnetofluidics.

Chapter 2 presents a comprehensive review on different techniques, phenomena and applications in micro magnetofluidics. The schemes reviewed include MHD and magnetic particles-based manipulation which can be categorized as ferrohydrodynamics (FHD), magnetorheology (MR), and magnetophoresis (MP) according to types of the magnetic fluids.

Chapter 3 reports experiments on droplet deformation under a uniformly induced magnetic field. Droplet geometry is analyzed as a function of flux density and thus the magnetization. Discussion on the results is carried out. The numerical model is established based on particle level-set method to solve the multiphase problem. A 
uniform staggered grid is used. In addition, comparison between experimental data and numerical results is accomplished by analyzing droplet dimension as a function of the magnetic Bond number. The effect of nonlinear magnetization during the experiment procedure is qualitatively observed.

Chapter 4 describes the experimental setup for manipulating a discrete sessile droplet dispensed on a hydrophobic solid surface. Discussion is carried out concerning the effect of a permanent magnetic field on droplet wetting and sliding. By considering the force balance, scaling analysis is achieved to study critical velocities for droplet motion.

Chapter 5 reports the experimental and numerical results for spreading of ferrofluid stream inside a conventional rectangular microfluidic channel. The width of the ferrofluid stream is analyzed in terms of different magnetic flux density.

Chapter 6 presents the experimental and numerical results for mixing efficiency of magnetic nanoparticles inside a circular chamber. Parameters such as magnetic flux density, viscosity ratio and flow rate ratio between magnetic and diamagnetic liquids are applied for the effect on the performance of the device.

Chapter 7 concludes of the current work and proposes future work. 


\section{CHAPTER 2}

\section{LITERATURE REVIEW}

Magnetic forces can be applied in microfluidics by using electrically conducting fluids or introducing magnetic particles into the diamagnetic working fluids. For electrically conducting fluids under an external magnetic field, the mutual interactions between the magnetic field and electric currents will generate the Lorentz body forces which can be used as the driving force. This leads to the domain of magneto-hydrodynamics (MHD). In a magnetic fluid under an external magnetic field, magnetic forces exert on individual magnetic particles. The volumetric magnetic force depends on the magnetic force on each particle as well as the particle concentration. According to the particle size, the magnetic fluid can be categorized as a fluid with discrete magnetic particles, the magnetorheological fluid and ferrofluid [1]. For a fluid with discrete magnetic particles, the particles need to be considered individually because the particle diameter is in the order of several microns or larger. A magnetorheological fluid (MR fluid) consists of smaller particles with diameter ranging from $10 \mathrm{~nm}$ to $10 \mu \mathrm{m}$. The MR fluid shows the magnetoviscous behaviour as the particles interact and react to a magnetic field. In the ferrofluid, the magnetic particles are less than $10 \mathrm{~nm}$ in diameter and can be well dispersed in the carrier fluid due to the dominant thermal energy.

The research on micro magnetofluidics concerning with the interactions between magnetism and fluid flow has been reviewed by Nguyen [1]. On the one hand, microfluidics allows the investigation of magnetically induced phenomena which is 
not possible in the large scale. On the other hand, magnetism provides a convenient and wireless way for control and manipulation of fluid flow on the microscale. Investigation of magnetofluidic phenomena in a microfluidic device has the advantage of a well-defined experimental condition. The small dimension of microchannels allows the generation of a large shear stress. The small device size also allows the simple implementation of a precisely controllable and uniform magnetic field. The low Reynolds number involved in microchannel allows experiments with negligible inertial force, simplifying the experimental conditions.

This review attempts to summarize the application of magnetism in microfluidic devices. The field of microfluidics is divided into continuous and digital microfluidics. The techniques are generally categorized according to their individual mechanism.

\subsection{Magnetohydrodynamics (MHD)}

In micro devices, there has been a growing interest in manipulating fluids to achieve various processes, such as chemical reaction and biological handling. Active approaches have been commonly adopted because of the effective working principle. For example, electrostatic forces have been used to transport liquids. However, the electrostatic-based method will result in unnecessary heating effect and require high electric potentials. The development of MHD is inspired by the discovery (Ritchie [2]) that conducting fluid can be pumped by applying an electric field together with a perpendicular magnetic field. MHD offers a flexible and effective way with lower cost. Comparing with conventional micropumps, MHD micropumps use active principles 
without moving parts, and the operating voltages are low. The flow rates generated by MHD pumps are comparable to most of the widely used pumping methods. The options of liquid are limited to liquid metals, hot ionized gases (plasmas) and strong electrolytes because of the requirements on electrically-conducting and non-magnetic properties [3]. In microfluidic domain, most of the working fluids in MHD-based devices are electrolytes (with low conductivity).

In general, MHD devices utilize orthogonal arrangement of the magnetic and electric field. The resulting Lorentz force causes the motion of the liquid. The technique of MHD is well-established and the MHD-based actuation mechanism has been applied in many microfluidic devices, such as stirrer, mixer and thermal reactor. The underlying physics as well as the various applications of MHD have been reviewed by Qian and Bau [4]. The governing equations for a conducting fluid under driving of the Lorentz force are,

$$
\begin{gathered}
\frac{\partial \rho}{\partial t}+\nabla \bullet(\rho \vec{u})=0 \\
\frac{\partial}{\partial t}(\rho \vec{u})+\nabla \cdot(\rho \vec{u} \vec{u})=-\nabla P+\nabla \cdot\left[\eta\left(\nabla \vec{u}+\nabla \vec{u}^{T}\right)\right]+\vec{J} \times \vec{B}
\end{gathered}
$$

where $\rho$ is liquid density, $t$ is time, $\vec{u}$ is velocity, $\eta$ is liquid viscosity, $\vec{B}$ is magnetic flux density and $\vec{J}$ is the electric current density for moving conductors which can be represented by a function of $\vec{B}$,

$$
\vec{J}=\kappa(\vec{E}+\vec{u} \times \vec{B})
$$

where $\kappa$ represents the electrical conductivity. 
For conventional MHD with working fluid having the electrical conductivity $\kappa$ and permeability $\mu_{0}$, the magnetic Reynolds number $\operatorname{Re}_{m}$ offers an estimation on the effects of magnetic advection to magnetic diffusion,

$$
\mathrm{Re}_{m}=\frac{u l}{\lambda}
$$

where $u, l$ and $\lambda$ are the flow velocity, the characteristic length scale and the magnetic diffusivity which can be defined as $\lambda=1 /\left(\mu_{0} \kappa\right)$. In the case of $\operatorname{Re}_{m} \gg 1$, magnetic flux line will be advected with the fluid flow. In the case of $\operatorname{Re}_{m} \ll 1$, the magnetic flux will not be affected significantly. In conventional magnetomicrofluidics, the typical magnitude of magnetic Reynolds number is in order of $10^{-8}$ which is much smaller than that of sun spot $\left(10^{8}\right)$, earth core flow $\left(10^{2}\right)$ and macroscale flow $\left(10^{-3}\right.$ to $\left.10^{-1}\right)[1]$

To characterize the importance of the Lorentz force relative to inertial force, the dimensionless interaction parameter (Stuart number) $N$ is introduced as,

$$
N=\frac{H a^{2}}{\operatorname{Re}}=\frac{\kappa B^{2} l}{\rho u}
$$

where Hartmann number $\mathrm{Ha}$ is another important dimensionless number for MHD which characterizes the importance of Lorentz force relative to viscous force,

$$
H a=B l \sqrt{\frac{\kappa}{\eta}}
$$

where $\eta$ is the dynamic viscosity of the working fluid. For a flow in a rectangular cross section $h \times w$, the velocity distribution $u\left(x_{2}, x_{3}\right)$ is derived as [5], 


$$
u\left(x_{2}, x_{3}\right)=-\frac{4}{\pi} \sum_{n=1}^{\infty} \frac{1}{1+2 n}\left[1-\frac{\cosh \left(\lambda_{n}\left(\frac{h}{2 w}-x_{2}\right)\right)}{\cosh \left(\lambda_{n} \frac{h}{2 w}\right)}\right] \sin \left((2 n+1) \pi x_{3}\right)
$$

where $\lambda_{n}^{2}=H a^{2}+(2 n+1)^{2} \pi^{2}$. When $H a=0$, the velocity distribution is the same as that of the pressure-driven Poiseuille flow in a rectangular channel.

\subsubsection{Governing equations of electric and magnetic fields}

The behaviour of electric fields and magnetic fields can be solved by the Maxwell's equation. The fluid is magnetized in a magnetic field. The straightforward definition of magnetization $\vec{M}$ is the quantity of magnetic moment $\vec{m}$ per unit volume in the magnetized medium,

$$
\vec{M}=\vec{m} n_{m}
$$

where $\vec{M}, \vec{m}$, and $n_{m}$ are the magnetization, the magnetic moment, and the number density of magnetic moments, respectively. The magnetic field strength $\vec{H}$ can be defined as,

$$
\vec{H}=\left(1 / \mu_{0}\right) \vec{B}-\vec{M}
$$

where $\mu_{0}=4 \pi \times 10^{-7} N / A^{2}, \vec{B}$, and $\vec{H}$ are the magnetic constant indicates the permeability of free space, the magnetic flux density and the magnetic field strength.

The magnetic field $\vec{B}$ is governed by Gauss's law for magnetism which is presented in a differential form,

$$
\nabla \cdot \vec{B}=0
$$

There will be polarization of conducting and non-magnetic fluids in an electric field. In a homogenous linear and isotropic dielectric medium, the polarization $\vec{P}$ can be 
defined as the difference between the imposed electric field strength $\vec{E}$ and the electric displacement field $\vec{D}$ which is a function of the electric susceptibility $\chi_{e}$,

$$
\vec{P}=\varepsilon_{0} \chi_{e} \vec{E}
$$

where $\varepsilon_{0}=8.854 \times 10^{-12} \mathrm{~F} / \mathrm{m}$ and $\chi_{e}$ are the electric constant indicates the permittivity of free space and electric susceptibility. The electric flux density $\vec{D}$ can be defined as,

$$
\vec{D}=\varepsilon_{0} \vec{E}+\vec{P}=\varepsilon_{0}\left(1+\chi_{e}\right) \vec{E}
$$

The governing equation of electric field is Gauss law which is given in a differential form as,

$$
\nabla \cdot \vec{D}=\rho_{f}
$$

where $\rho_{f}$ is the free charge density.

\subsubsection{MHD-based Micropumps}

The MHD-based pumps have been realized and demonstrated to be suitable and efficient for acting as power sources in the fluid circulation [6-7]. The non-intrusive property offers compatibility for biological utilization as many of the biological solutions are slightly conductive.

\subsubsection{DC-MHD pump}

The first DC-MHD micropump was reported by Jang and Lee [8] with both theoretical and experimental studies. The performance of the micropump was characterized with pressure head difference and flow rate of the conducting liquid at different current 
supply. In the presence of a magnetic flux density at $0.44 \mathrm{~T}$, a pressure head difference of $18 \mathrm{~mm}$ could be achieved at $38 \mathrm{~mA}$. Under the same magnetic flux density, the flow rate was $63 \mu \mathrm{L} / \mathrm{min}$ at $1.8 \mathrm{~A}$ inside the inlet/outlet tubes with $2 \mathrm{~mm}$ in diameter. Huang et al. [9] investigated the propulsion of several aqueous solutions in this type of MHD micropump shown in Figure 2-1. They studied on one-dimensional model consisted of the continuity equation and volumetric force balance established by Newton's law with gravitational force exempted for simplification. Numerical computations on the dynamic behaviour of the MHD micropump were implemented by 20SIM which was a commercial software based on the bond graph theory. In order to verify the numerical results, experiments were conducted with a DC type MHD micropump fabricated with Lithographie, Galvanoformung, Abformung (LIGA) technology to form a cross-sectional area and chamber length at $500 \mu m \times 800 \mu m$ and $20 \mathrm{~mm}$, respectively. Neodymium-iron-boron $(\mathrm{NdFeB})$ permanent magnets were used to provide the magnetic field with a flux density at $1.08 \mathrm{mT}$. The comparison between the experimental and numerical results was carried out on liquid flow rate which was proved to be proportional to the supplied voltage. The generation of bubble was observed at the electrodes in the experiment with a relatively high voltage supply $(15 \mathrm{~V})$. Bubble generation due to electrolysis effect of conducting liquid would result in disruption or even block of the flow. The MHD based propelling principle was later applied in a thermosyphonic system [10-11]. 


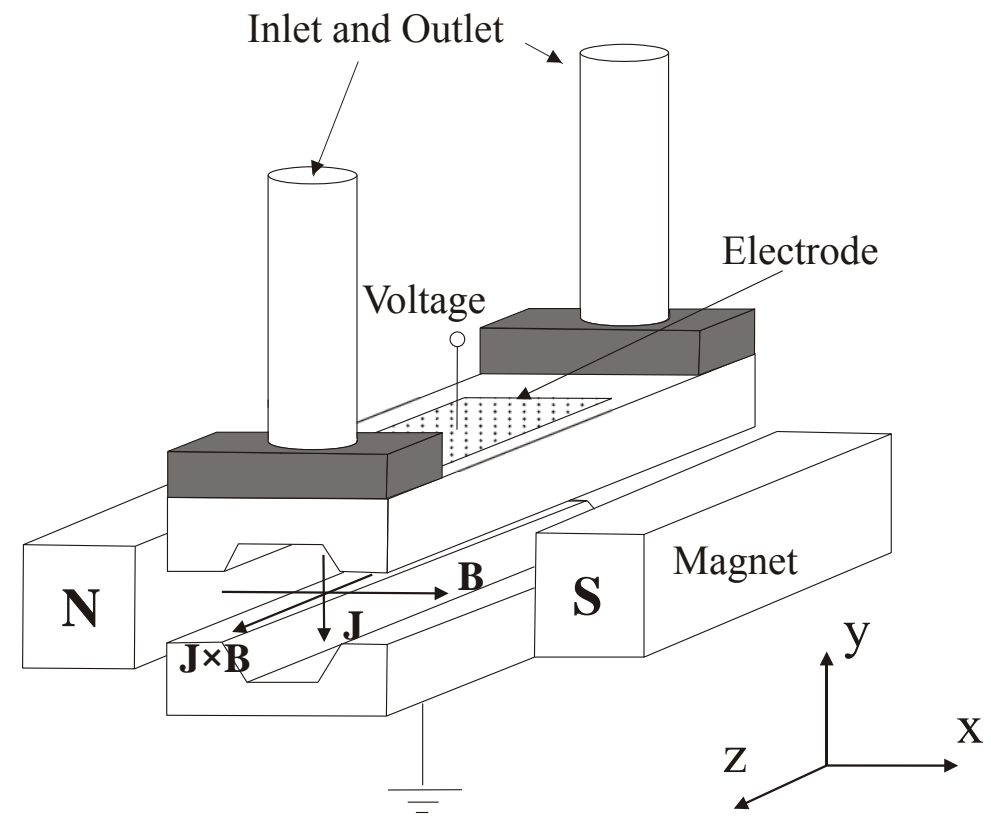

Figure 2-1 Inclined view of the first MHD pump [8]

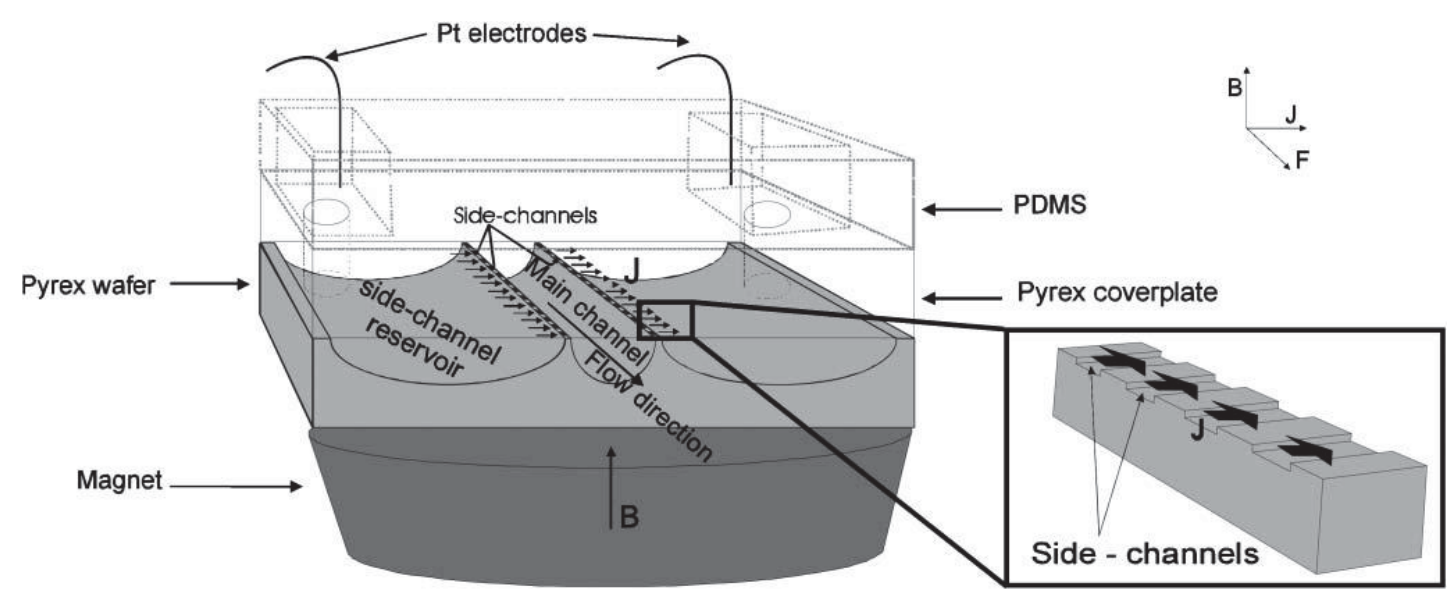

Figure 2-2 The channel configuration and electrodes arrangement [12]

Since the proposal of the MHD pumping principle by Jang and Lee [8], efforts were devoted to improve the performance of the DC-MHD micropumps. As the generation of bubbles was inevitable in the DC-MHD pumps, the main concerns were the control of the bubble size, location and aggregation rate. By judicious design for isolation and release, the flow impediment due to bubble generation at the electrodeelectrode interface could be controlled even at high DC current density. Homsy et al. [12] provided a new DC pump design with the electrodes and thus the generated 
bubbles outside the main channel. The bubbles would escape into the atmosphere rather than entering the working channel. The channel configuration and electrodes arrangements were presented in Figure 2-2. The integration of the pump with planar NMR coils was achieved in a microfluidic system for nuclear magnetic resonance (NMR) analysis [13]. The experiments on NMR showed that the device was capable to work well under a high DC magnetic flux density of $7 \mathrm{~T}$. However, the possible maximum flow rate was low $(0.5 \mu \mathrm{L} / \mathrm{min})$. To overcome this problem, Nguyen and Kassegne [14] proposed a new design to prevent the coalescence of the bubbles and thus minimize the retarding effects on fluid flow.

The above-mentioned design of channels increased the complexity and cost in microfabrication. The use of redox electrolytes were preferred to enable the obviation of issues such as the bubble formation, the electrode corrosion, and electrolyte depletion. In a solution, the redox species carried currents and made the liquid highly conducting because of oxidation and reduction. The redox electrolytes would undergo reversible electrochemical reactions at the surface of the electrodes in MHD pumps. The redox MHD-based pump was studied and reported by many research groups [1518]. The redox-MHD pumping scheme was a popular application of magnetic field into the electrochemical process. With an imposed magnetic field, the mass transport of electrolyte would be increased due to the augment of the MHD body force [19-21]. The steady-state mass-transport-limited current generated during the faradaic process depended on the impact factors such as the electrode area, the magnetic field strength, 
the diffusion coefficient, the bulk concentration of the redox-active species and the kinematic viscosity of the electrolyte [22].

Arumugam et al [23] proposed an experiment to verify the concept of redox-MHD propelling in microfluidic device. The micro channels were fabricated with low temperature co-fired ceramic tapes (LTCC) with electrodes positioned on the opposing walls along the length of the channels. Single and paired NdFeB permanent magnets were applied to provide the external magnetic field. The flow velocities up to 5.0 $\mathrm{mm} / \mathrm{s}$ were achieved for a $0.5 \mathrm{M} \mathrm{NB}$ solution with electric current density and magnetic flux density at $6 \mathrm{~mA}$ and $0.41 \mathrm{mT}$. Grant et al. [24] reported an experimental work of MHD-induced enhancement in the molecules mass transfer and the generation of different flow pattern which was potentially useful in chemical delivery.

Duwairi and Abdullah [25] developed 2D analytical and numerical models for MHD micropump with a rectangular geometric configuration. Starting from this analytical and numerical work, a 2D numerical model was developed for the redoxMHD pump by coupling the mass transport and momentum governing equations [26]. For the redox-MHD pump, the transport of ions was required to be considered as the intensity of the electric current in electrolyte solutions was determined by the concentration of the redox species. To reduce the assumptions in the numerical investigation, another numerical work was presented by Qian and Bau [27] for a redox-MHD pump with a planar conduit. For the purpose of a more comprehensive understanding of the redox-MHD flows, a 3D prediction was obtained with a theoretical model [28]. The numerical calculation was carried out with the commercial 
finite element package COMSOL. The steady state flow field and ionic concentration distribution was obtained with various potential differences, initial bulk concentrations of the redox and the magnetic flux densities. The induced electric current and flow rate was obtained from the $3 \mathrm{D}$ model and compared with the experimental results from the literature and the 2D model published previously [27]. For a more practical resolution useful for design and optimization, flow rate and average velocity were derived based on a closed form approximation. To validate the full 3D model and 3D approximation, average velocity was compared with the results of the $2 \mathrm{D}$ model as well as the experimental results from Aguilar et al [29]. The 3D approximation was further validated by comparing the predictions with the experimental results from Arumugam et al. [23] and Ho et al. [30]. With considering the effect of electric double layer and Joule heating, Patel and Kassegne [31] reported the analytical investigation on the multi-physics of the MHD micropumps and the numerical framework for a full 3D solution.

\subsubsection{AC-MHD pump}

In order to prevent the flow impediment and electrode degradation issue in high-DC MHD principle, the AC MHD micropump has been proposed and studied with bidirectional pumping capability. By applying an AC current with adequately high frequency, the frequent chemical counterreaction refrains from net reactant migration leading to electrode and reactant degradation and bubble generation. 
In the case of MHD using AC current synchronized with an AC magnetic field, the time-averaged Lorentz force is a function of amplitude of electric-flux density, magnetic-flux density and the phase angle between them. In word, the Lorentz force is controllable for both amplitude and direction. For the specific case with sinusoidal wave, the Lorentz force $\vec{F}_{L}$ and the electric current density $\vec{J}$ can be expressed as [32],

$$
\begin{gathered}
\vec{F}_{L}=\vec{J} \times \vec{B} \sin (\omega t) \\
\vec{J}=\kappa(\vec{E} \sin (\omega t+\varphi)+\vec{u} \times \vec{B} \sin (\omega t))
\end{gathered}
$$

where $\kappa$ is the conductivity, $\omega$ is the angular frequency of the waves, and $\varphi$ is the phase angle between the electric and magnetic fields. For the requirements of unidirectional propulsion, the direction of Lorentz force can remain unaltered by properly synchronizing the magnetic field and the AC current. There is a model introduced to describe the MHD flow based on Poiseuille flow theory.

The above-mentioned one-dimensional analysis based on Poiseuille flow theory [32] was not capable of illustrating the effects of channel sizes and shapes as the frictional effects of the walls were exempted. In addition, the induced electric current density was omitted in the analysis as well as the induced reverse Lorentz force. Consequently, the theoretical predictions would show discrepancy with the experimental measurements. The alternative was presented by Wang et al. [33] with simplified flow model based on steady state, incompressible and fully developed 2D laminar flow theory. The numerical computation was implemented with the central difference scheme based on explicit finite difference method (FDM) and agreed well with the other experimental results. According to the prediction with $\mathrm{NaCl}$ aqueous 
solution, flow rate at $1.3 \mathrm{ml} / \mathrm{s}$ could be achieved when magnitude of electric current and magnetic flux density were $0.6 \mathrm{~A}$ and $0.4 \mathrm{~T}$ respectively. For verification, the numerical computation was implemented with the same parameters as the experimental investigation of Lemoff and Lee [32].

In a conventional rectangular conduit, an AC MHD pump exhibited longer operation hour and allowed liquid transport at the velocity up to $20 \mathrm{~mm} / \mathrm{s}$ [34]. In order to resolve the issue with high current, lower current densities $\left(<8 \mathrm{kA} / \mathrm{m}^{2}\right)$ and higher magnetic fields (70 mT) were utilized in AC MHD devices. The theoretical and Finite Element Method (FEM) numerical investigations were reported as well as the experimental verification. For flow in a channel with cross-section area of $6.5 \mathrm{~mm}^{2}$, average velocity could be achieved up to $22 \mathrm{~mm} / \mathrm{s}$. Similarly, Sadler et al. [35] reported the numerical work by using CFDRC ACE+ TM finite element analysis software together with the experimental verification. With assuming zero phase shifts between the applied sinusoidal electric field and the generated electric current density, the volumetric flow rate $Q$ was derived as,

$$
Q=\frac{\vec{J} \vec{B} w^{3} h^{2} \cos \varphi}{8 \eta l(w+h)^{2}}
$$

where $l, w$, and $h$ were the length, width and height of the working channel. It was worth noting that bi-directional flow was achievable with opposing flow directions determined by the sign of the cosine function. To verify the theory with the experimental study, a ceramic AC type MHD micropump was fabricated and tested with the current wave at high frequency $(1 \mathrm{kHz})$. The resolve of bubble generation issue was demonstrated by a long term investigation on the flow impedance. 
Annular channel geometry gained much interest for pumping with MHD concept because of its property of free pressure head which could facilitate the characterization of the system function by flow measurements. The mathematical model for MHDinduced fluid circulating in an annular channel was developed by Gleeson and West [36]. With assumption, the momentum equation could be reduced to a 2D Poisson equation for the azimuthal velocity which could be solved by the Fourier-Bessel expansion or numerical computation. To validate the mathematical model, an MHDbased microreactor was fabricated and tested [37]. The microreactor was fabricated on a silicon substrate. The high frequency of the applied AC current $(1 \mathrm{kHz})$ ensured a continuous viscous flow and the synchronization between the electric and magnetic field. When operating with $1 \mathrm{M} \mathrm{KCl}$ electrolyte, the orders of velocities and volumetric flow rates were $0.6 \mathrm{~mm} / \mathrm{s}$ and $3.1 \mu \mathrm{L} / \mathrm{min}$, respectively. The AC-MHD propulsion concept together with the annular channel configuration was used for amplification of DNA by the polymerase chain reaction (PCR) by West et al. [38], and circular chromatography by Eijkel et al. [39-40].

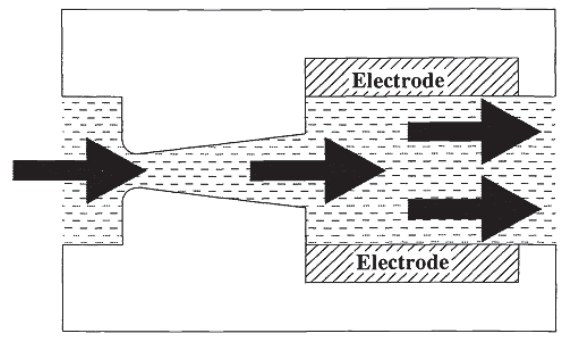

(a)

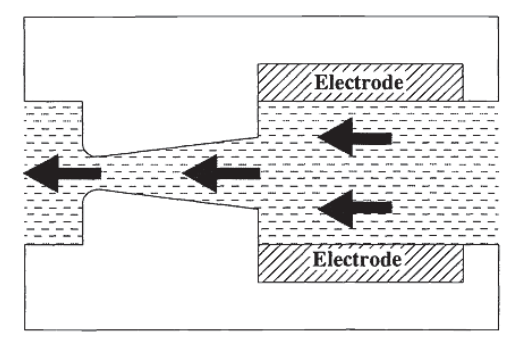

(b)

Figure 2-3 The illustration of operation mode of an AC type MHD micropump with diffuser/nozzle structure: (a) diffuser mode; (b) nozzle mode. [41] 
On the other hand, the diffuser/nozzle structures were adopted in the AC type MHD micropump [41-42]. This configuration was preferred occasionally due to the high cost of generating an electromagnetic field with high strength and density. The study started from the DC micropumps reported by Huang et al. [9]. The same permanent magnet was used to provide the external magnetic field. However, an alternative voltage was applied to the electrodes to resolve the issue of bubble generation. During one period of the wave, liquid could be driven back and forth in the channel. The diffuser and nozzle mode were shown in Figure 2-3(a) and (b). Due to the significant higher resistance in the nozzle direction, a net flow was produced in the diffuser direction as larger volume of liquid is pumped. Bubble generation was greatly reduced or prevented because of the back and forth movements of ions across the electrodes.

\subsubsection{MHD-based Mixers and stirrers}
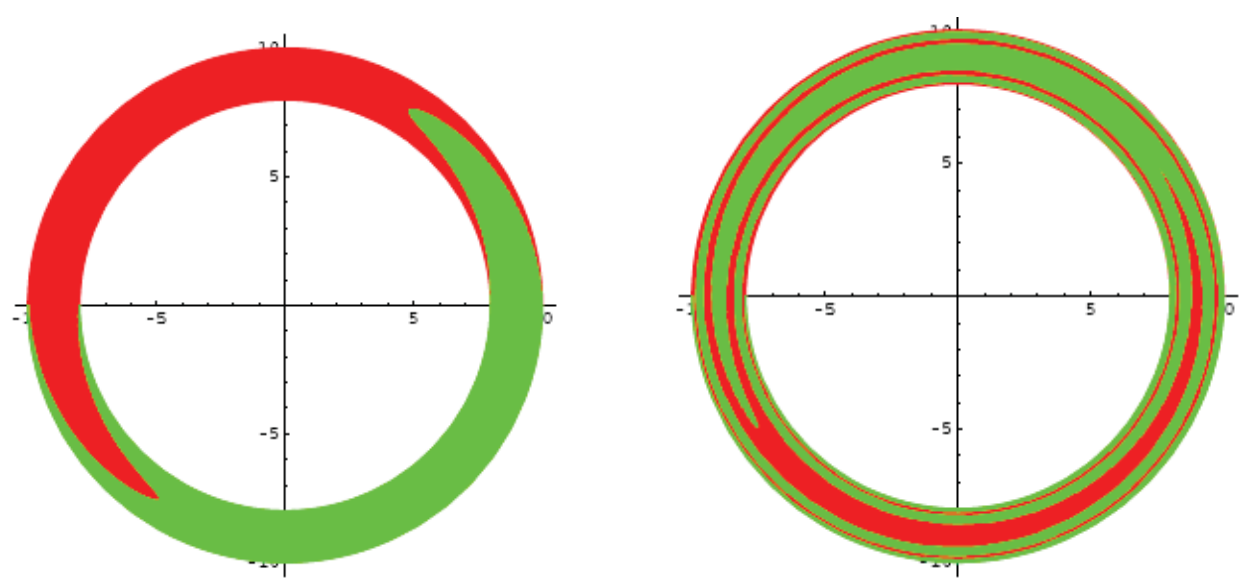

Figure 2-4 The growth of interface in an annular channel [36]

MHD has also been explored for its feasibility in mixers and stirrers. In typical microfluidic devices, fluid flows in a laminar way. The installation of moving parts for 
propelling will increase the cost, uncertainty and complexity. Consequently, the mixing efficiency and throughput are limited to hydrodynamic concepts. To meet the requirements of homogenous liquid for some chemical processes, a number of techniques have been explored and tested to achieve a thorough mixing in microchannels.

MHD was demonstrated to be suitable for mixing application in an annular channel with Lorentz force driving the liquid flow and consequently increasing the interfacial area for convection-diffusion mixing [36]. The velocity in the center of the annular channel was higher than that at the sides. As shown in Figure 2-4, two initially separated liquids were forced to pass through each other. The diffusion-mediated mixing in a laminar flow could be realized by the increase of interfacial area due to liquid circulation. In the presence of MHD driven force, a simplified 2D model was developed to estimate the interface length as a function of time. To utilize the MHD propulsion, a vertical magnetic field was generated by electromagnets attached to the electrodes on inner and outer walls of the channel. Meanwhile, a radial electric field can also be obtained by applying a potential difference across the inner and outer electrodes. The discussion on the $2 \mathrm{D}$ mathematical modeling and finite element method (FEM) numerical simulations of laminar convection-diffusion problem was reported by Gleeson et al. [43]. Gleeson [44] also reported a closed-form approximation and numerical calculation for prediction of the mixing time. This annular micromixing model was later proved by experimental investigation of mixing between Bromophenol blue (BPB) and fluorescein [37]. 
In addition, MHD-based principle was used for mixing by inducing a secondary flow which would result in the chaotic advection of the fluid in a conventional straight channel. The direction and magnitude of the electric currents was flexible with judicious patterning of electrodes. As a result, the complicated flow patterns can be realized by Lorentz force with various direction and magnitude. By varying the arrangement of the electrodes, cellular convection of the electrolyte solution was achieved for chaotic mixing in LTCC channels integrated with arrays of electrodes [45]. As shown in Figure 2-5, currents in opposing directions were generated in two adjacent pair of electrodes. Consequently, the resulting Lorentz forces had opposite directions in alternative pair of electrodes. The experimental investigation was carried out and the results agreed well with the theoretical prediction. Xiang and Bau [46] considered two groups of electrodes with every electrodes cladding by two electrodes from the other group. The two groups of electrodes were activated alternately at different frequency. Both the experimental and mathematical investigations were reported with comparison on streamlines with only one group of electrodes activated.

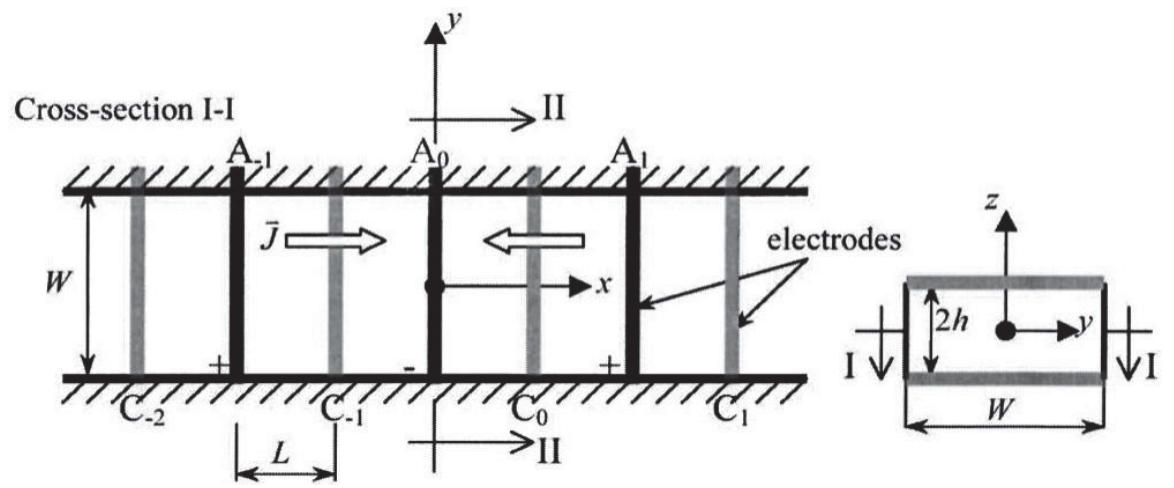

Figure 2-5 Depiction of the pattern of the electrodes and the generated electrical current [4546]. 
Yi et al. [47] proposed a DC MHD-based stirrer with conducting liquid filled cavity confined between two eccentric cylinders acting as electrodes. With a potential difference imposed between the inner and outer electrodes together with a magnetic field parallel to the cavity axis, flow circulation could be formed and centered next to the inner electrode. Mathematical model was established for solving the flow field in the cavity between two cylinders, outer cylinder with one "dot" inner electrode, and outer cylinder with two "dot" inner electrodes. In experiments, a metal washer worked as the outer electrode and copper wires were served as the inner electrodes. A crude qualitative agreement was achieved between the experimental investigation and mathematical prediction.
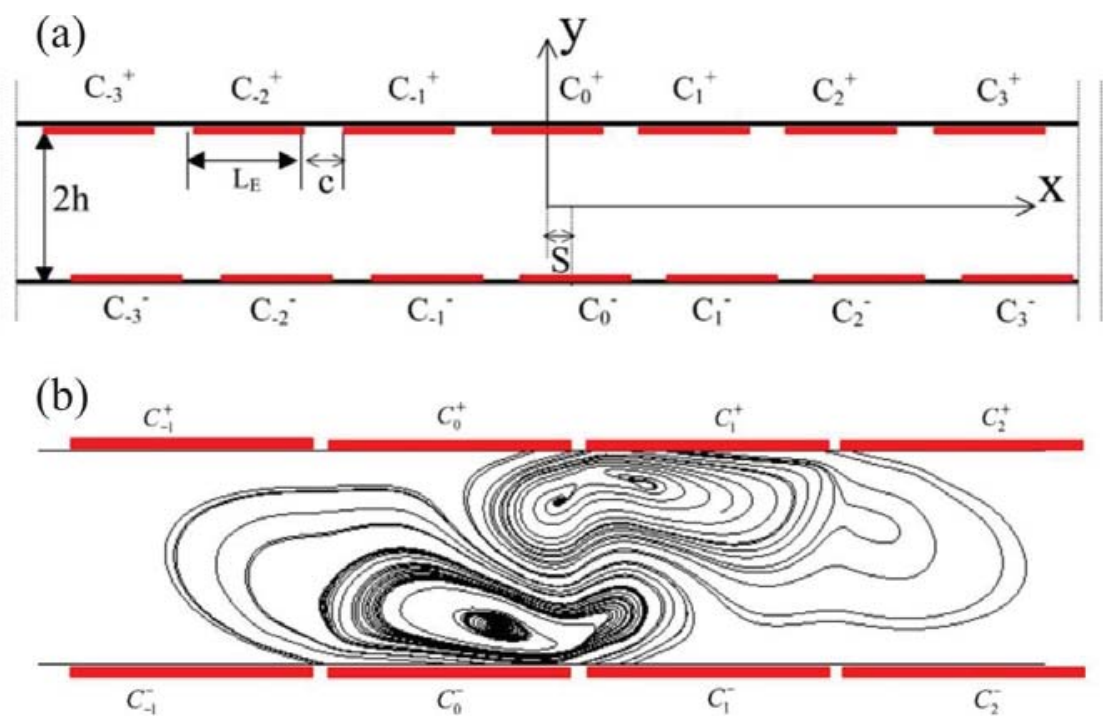

Figure 2-6 Non-intrusive MHD-stirrer (a) the arrangement of electrodes with displacement shift S; (b) flow pattern. [48].

Qian and Bau [48] proposed a new design of DC-MHD pump without inner electrodes. Numerous identical electrodes were deposited uniformly on the opposite surfaces of the conduit. Considering a rectangular conduit, two diagonally positioned electrodes were activated to enhance mixing with a secondary flow by generating 
Lorentz force with a component transverse to the conduit's axis. The alternative was the activation of staggered top and bottom electrodes (with a length at $L_{E}$ ) with a displacement $S\left(0 \leq S \leq L_{E} / 2\right)$ as shown in Figure 2-6(a). The potential distribution was solved by using the augmented system, while the flow field was numerically computed by a commercial software CFD-ACE. As shown in Figure 2-6(b), the numerical computation of concentration distribution focused on flow patterns without displacement for top and bottom electrodes. To achieve the perturbation and propagation of liquid simultaneously, the electrodes were wired together and activated diagonally. An average axial velocity of $5 \mathrm{~mm} / \mathrm{s}$ could be achieved with a potential difference at $2.5 \mathrm{~V}$ under a magnetic field with flux density at $0.4 \mathrm{~T}$. In order to obtain a more complicated flow, the electrode pairs could be activated alternately. Using the same parameter, experiments were carried out and proved to be in good qualitative agreement with the numerical prediction.

\subsubsection{MHD-based pump in microfluidic network}

In addition to the operation as a stand-alone device, multiple MHD pumps could be integrated on a single chip for complicated microfluidic flowing system.

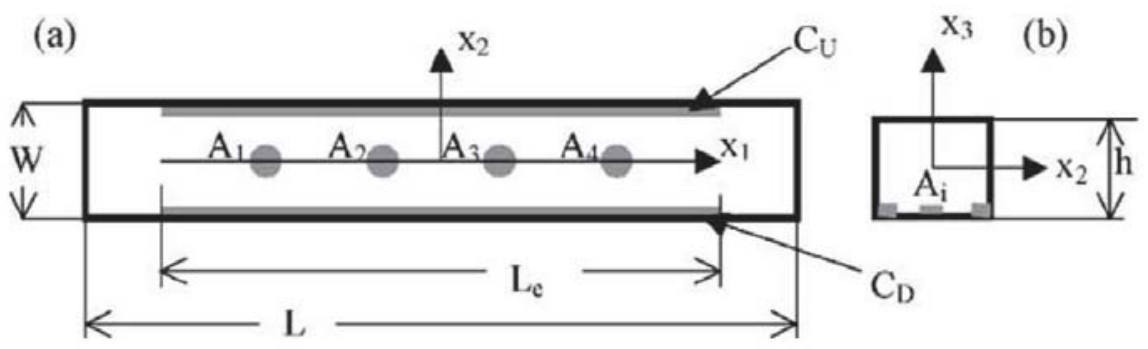

Figure 2-7 The branch of the microfluidic device: (a) top view; (b) side view. [49] 
MHD was explored with the application in a multi-task microfluidic network. The basic element of the microfluidic network consisted of a channel and two electrodes deposited at the opposite side of the channel walls. A microfluidic network was formed with many basic elements being connected together. With suitable patterns of electrodes, both the task of pumping and stirring liquids could be realized for control in the basic element (branch) of the fluidic network [49]. The typical arrangement of the electrode was shown in Figure 2-7. In the operation mode of stirring, circulatory motion of fluid could be achieved and centered at the "dot" electrode. To increase the complexity of the chaotic advection, a series of "dot" electrodes could be used and activated in turn. Both the theoretical prediction and experimental test were provided for validation. Qian et al. [50] reported an improvement of the the above-mentioned arrangment of electrodes to facilitate the integration of the branches into the MHD network. The mathematical model was established to obtain the singular solution of the 2D MHD Stokes problem for the case of one "dot" electrode confined between two parallel plane electodes. With multiple "dot" electodes activated alternately, the quasistatic approximation was adopted to compute the flow field. The experimental investigation was presented and qualitatively agreed well with the theoretical predictions.

The switch of flow was proved and experimentally investigated for a Y-shaped fluidic circuit. Outside of the channel, two AC MHD pumps were installed on different arms. The flow could be switched between the branches by activating the micropump 
on one arm, and giving the other one a counteracting pressure to prevent flow. The flow speed of switching in Lemoff and Lee's design was $0.3 \mathrm{~mm} / \mathrm{sec}$ [51].

\subsection{Magnetic particles-based applications}

For non-conducting liquid, magnetic force can be utilized by introducing magnetic particles. According to the particle size, a magnetic fluid is categorized as a fluid with discrete magnetic particles, a magnetorheological fluid and ferrofluid [1]. Correspondingly, micro magnetofluidics using magnetic fluids can be categorized as magnetophoresis (MP), magnetorheology (MR), and ferrohydrodynamics (FHD).

In a magnetic field, the paramagnetic particles became magnetic dipoles. Pankhurst et al. [52] reviewed the underlying physics of magnetic nanoparticles under an external magnetic field. Magnetic nanoparticles have been extensively used in medical and biomedical assays. Magnetic particles isolated from magnetotactic bacteria were used for enzyme immobilization [53]. The detection and enumeration of genetic variations for DNA molecules were realized by using fluorescent magnetic particles [54]. In molecular biology and biochemistry, magnetic particles were used for separation and extraction of cells and DNAs [55]. Microfluidic mixing was achieved by using magnetic beads under a local alternating magnetic field [56]. Effective mixing with an efficiency on the order of $95 \%$ was achieved by tuning the magnitude and the frequency of the field. Furthermore, the capability for a single-step immunoassay was proved by investigating the changes in Brownian rotation dynamics of magnetic particles [57]. Shinkai [58] reviewed the medical application of magnetic 
particles including separation, immunoassay, magnetic resonance imaging (MRI), drug delivery and hyperthermia enhancement. By filling nanotubes with paramagnetic nanoparticles, magnetic tubes could be realized with numerous potential applications [59].

\subsubsection{Magnetically-induced manipulation of liquid in digital microfluidics}

Digital microfluidics is an emerging research field in today's microfluidics. The digital microfluidic device is an open structure with discrete liquid droplets dispensed directly on a solid surface. The absorption of liquid on the solid wall would be reduced in this configuration [60]. Moreover, the open structures offer lower cost, less sample consumption, easier fabrication/assembling and reconfiguration, mainly because of eliminating the requirement of micro-pumps or valves.

Liquid will spread and wet the surface after been dispensed. The shape of a sessile droplet depends on properties of the surface, the liquid and the surrounding medium. In digital microfluidics, a major goal is the control of phenomena which affect the wetting condition. The tunable wetting condition of liquid enables the manipulation of a small amount of liquid on a surface. Controlled actuation of liquid droplets on a planar surface is of utmost importance for digital microfluidics. A droplet can work as a vehicle for transporting reagents. The confined space in a droplet represents an ideal reactor, and a high throughput platform for many chemical and biochemical processes. A planar surface allows the implementation of 2D droplet-based microfluidic systems which can handle droplets individually. Consequently, one of the most critical issues 
considered in digital microfluidics is the way to induce the droplet motion on a surface. This approach demands not only an appropriately treated surface, but also a force for droplet manipulation.

A number of forces can be applied for droplet actuation, such as gravitational [6162], capillary [63-66], electrical [67-68], acoustic [63, 69-70], and magnetic forces [71-73]. Undoubtedly, the achievable velocity of the droplet is a critical issue for the evaluation of the applied method. A number of experimental works were carried out and reported on droplet manipulation. Yang et al. [74] presented a review on manipulation of droplets in microfluidics, including digital-microfluidic devices.

To facilitate and accelerate the development of this field, current research also emphasizes on simulation of droplet actuation using computational fluid dynamics (CFD). Numerical methods were developed for studying the microfluidic behaviours dominated by viscous force and surface tension. Droplet-based microfluidic problems are multiphase flow problems in term of numerical modeling. To this extent, the challenge was handling and tracking the interface which distinguished different fluids. Zheng and Zhang [75] applied the level-set method to simulate droplet spreading on a solid surface involving a phase change process due to solidification. Under the effect of a shear flow, sessile droplet rolling behaviour was calculated by using the contact line velocity in the reinitialization function of the level-set method [76-77]. Numerical methods were also developed for investigating droplet behaviour actuated by external forces. For example, numerical models were implemented to solve thermal actuation problems, such as rising of multiple bubbles [78] and droplet breakup behaviours [79]. 
Behaviour of both freely suspended droplets and sessile droplets was investigated numerically [80-81].

Magnetic actuation has exhibited unique advantages in many applications for manipulation of droplets. The method is robust and wireless. The magnetic actuation method is independent from the dielectric properties of the liquid which is beneficial as the ferromagnetic body has the ability to retain stable equilibrium state under the influence of a magnetic field compared to unstable situation of the electrostatic force. Magnetic manipulation also has a significant advantage of diversity of magnetic particles and easy control in miniaturized devices.

In most of the applications of digital microfluidics, magnetic forces were induced using magnetic particles. So far, the investigation mainly focused on liquid with discrete magnetic particles (magnetic beads) and ferrofluid.

\subsubsection{Magnetic Bead Cluster Based Actuation}

Magnetic particles were introduced in droplet as the force mediators for magnetic actuation. The magnetic beads formed cluster after being immersed in a magnetic field. Transportation was realized of a small amount of liquid. At the early stage, a simple theoretical model was built by considering the balance among magnetic force, friction force and the deformation-induced capillary force. The water droplet with magnetic beads was put into a container filled with silicon oil [82] or encapsulated in a thin layer of silicon oil [71]. A permanent magnet was used to provide the magnetic field. Only the horizontal components of the force vectors were considered in the model. 
The magnetic force per unit volume exerted on the bead cluster was defined as $\vec{F}_{m p}$,

$$
\vec{F}_{m p}=(\vec{M} \cdot \nabla) \vec{H}
$$

where $\vec{M}$ is the magnetization of the bead cluster and $\vec{H}$ is the magnetic field strength. As the droplet was driven horizontally, the effective magnetic force was the horizontal component. The Eq. (2.17) was partially differentiated to achieve $\vec{F}_{m p x}$,

$$
\vec{F}_{m p x}=\vec{M}_{x} \frac{\partial \vec{H}_{x}}{\partial x}+\vec{M}_{y} \frac{\partial \vec{H}_{x}}{\partial y}+\vec{M}_{z} \frac{\partial \vec{H}_{x}}{\partial z}
$$

where $\vec{H}_{x}, \vec{M}_{x}, \vec{M}_{y}$, and $\vec{M}_{z}$ are $x$ component of the magnetic field strength $\vec{H}$ and $x, y, z$ component of the magnetization $\vec{M}$. The magnetic force $\vec{F}_{m}$ was consequently estimated using the mass $m_{b}$ and density $\rho_{b}$ of the bead cluster,

$$
\vec{F}_{m}=\left(\frac{m_{b}}{\rho_{b}}\right) \vec{F}_{m p x}
$$

The friction force between the oil layer and the substrate $\vec{F}_{f}$ could be obtained by,

$$
\vec{F}_{f}=K_{f} r_{c} \eta_{o} \vec{u}
$$

where $K_{f}$ is the friction constant, $r_{c}$ is the radius of the contact area between the droplet and substrate, $\eta_{o}$ indicates the value of viscosity for the oil layer and $\vec{u}$ is the droplet velocity.

Capillary force was induced by droplet deformation when bead clusters pressed against the droplet surface under the dragging effect of the magnetic field. Assuming a bead cluster to be a single equivalent sphere, the length of the outer circumference of the sphere $L_{c}$ during the extraction was estimated as, 


$$
L_{c}=6^{\frac{1}{3}} \cdot \pi^{\frac{2}{3}} \cdot\left(\frac{m_{b}}{\rho_{b}}\right)^{\frac{1}{3}}
$$

The capillary force $\vec{F}_{c a p}$ was consequently given as,

$$
\vec{F}_{c a p}=\vec{\sigma}_{s w} L_{c}
$$

where $\vec{\sigma}_{s w}$ is the interfacial tension between silicone oil and water.

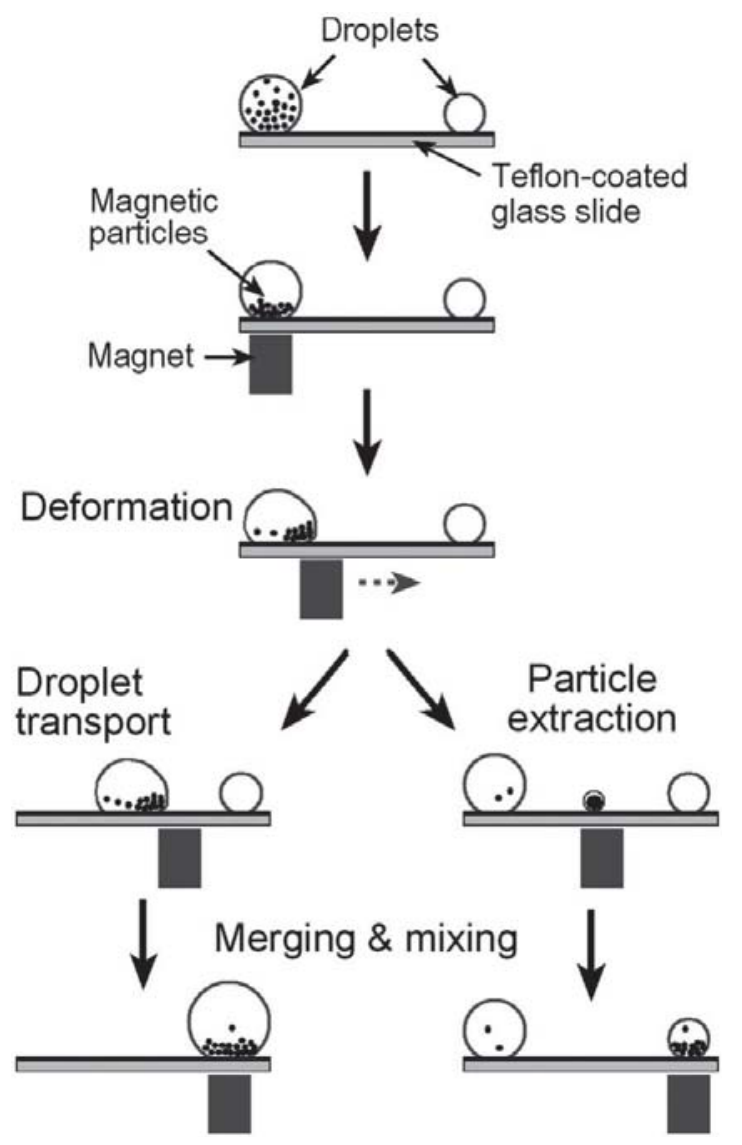

Figure 2-8 Schematic set-up and fundamental operations in a magnet-actuated droplet system [71].

Numerous experiments were conducted on droplet actuation based on the use of magnetic bead cluster. Many fundamental microfluidic procedures were achieved, such as deformation, transport, merging and mixing. In particular, the particles were possible to be extracted from the mother droplet. Corresponding with the abovementioned theoretical model, Long et al. [71] experimentally investigated the 
movement, coalescence, and splitting of droplets on a planar surface under the actuation of a permanent magnetic field. Magnetic beads were introduced into an oilcoated water droplet for magnetic actuation. The schematic setup and basic droplet operation was shown in Figure 2-8. García [83-84] presented the magnetic manipulation of hydrophobic droplets containing carbonyl iron magnetic particles. The displacement, coalescence and splitting of droplets were achieved in a sample container with controllable humidity. Two different surfaces with hydrophobic properties were applied to reduce resistance during the movement. Pipper et al. [85] applied the magnetic manipulation of an aqueous droplet in a clockwork PCR. The droplet was encapsulated in mineral oil to reduce droplet evaporation and the friction between droplet and the bottom surface. For further application of magnetically driven device, droplet-based platform was used for enzymatic reaction [82]. Magnetic separation and fusion were experimentally investigated on this platform [86]. Besides, requirements for particles extraction were studied based on the total weights of the magnetic beads and the resulting interfacial tension.

\subsubsection{Actuation of Ferrofluid Droplets}

The most commonly used magnetic fluid is the ferrofluid which is synthesized with magnetic particles and a carrier fluid. Magnetic particles are dispersed stably in the carrier fluid and thermal Brownian motion of these particles strongly affects the properties of the resulting ferrofluid. In addition to the magnetic particles and carrier fluid, surfactants are coated on the particle surface to prevent the agglomeration of 
particles to each other. Since the discovery of ferrofluid in early 1960s, this material has been applied extensively. Readers may refer to Rosensweig [72] for detailed studies on the field stability, the significant parameters, the preparation of magnetic particles and the chemical properties of magnetic particles in a solvent. Vékás [87] reviewed recent achievements on the synthesis technology of magnetic particles and the preparation of ferrofluid. Numerous research works have been conducted on properties of ferrofluid as functions of the particle concentration [88], the magnetization [89-90] and the viscosity [91-92].

A ferrofluid droplet can be dragged by a magnet moving towards a given direction to achieve transport, merging, mixing and splitting. Magnetic actuation is generated by the interaction between magnetic nanoparticles suspended inside the droplet and an external magnetic field. Langevin's theory is favorable to systematically describe the super-paramagnetic properties in ferrofluid neglecting the interaction with particles. The magnetization law of ferrofluid can be expressed as,

$$
\begin{gathered}
\vec{M}=\vec{M}_{s}\left(\operatorname{coth} \xi-\frac{1}{\xi}\right)=\phi_{\mathrm{vol}} \vec{M}_{d}\left(\operatorname{coth} \xi-\frac{1}{\xi}\right) \\
\xi=\frac{\mu_{0} \vec{m} \vec{H}}{k_{B} T}
\end{gathered}
$$

where $\vec{M}_{s}$ denotes the saturation moment of the ferrofluid, $\vec{M}_{d}$ is the saturation moment of the magnetic nanoparticles in the bulk and $\phi_{\mathrm{vol}}$ represents the corresponding volume fraction of magnetic nanoparticles, $\xi$ stands for the energy ratio between the magnetic moment energy in the applied field and the thermal energy, 
$\mu_{0}=4 \pi \times 10^{-7} N A^{-2}$ is the magnetic constant indicates the permeability of free space, $k_{B}$ is the Boltzmann constant, and $T$ represents the absolute temperature. Elmore [93] has applied the above-mentioned equations to investigate the magnetization properties of ferrofluid under an external magnetic field.

The external magnetic field was flexible and programmable. Droplets dragged by the magnet could have different velocities. Bacri et al. [94-95] investigated the behaviour of a magnetic droplet under a static magnetic field. Droplet deformation and instability were investigated to predict its dependence on the field strength, the temperature, the particle size or the ionic strength. Particles agglomeration was observed in the presence of an applied magnetic field [96-97]. Instability was shown for the ferrofluid with anionic surfactant. Experiments were carried out to study droplet elongation as functions of field strength and time-dependent instability. For applications in printer [98], equilibrium shape of the ferrofluid droplet under a magnetic field was derived by minimizing potential energy with respect to the droplet height and base radius at constant volume.

\subsubsection{Configuration of Magnetic Field}

Different configurations were studied to provide flexible and programmable magnetic field. Permanent magnets were commonly utilized as simple and reliable sources of actuating magnetic field [99]. Magnetowetting phenomena and the sliding motion of a ferrofluid droplet were experimentally investigated. 


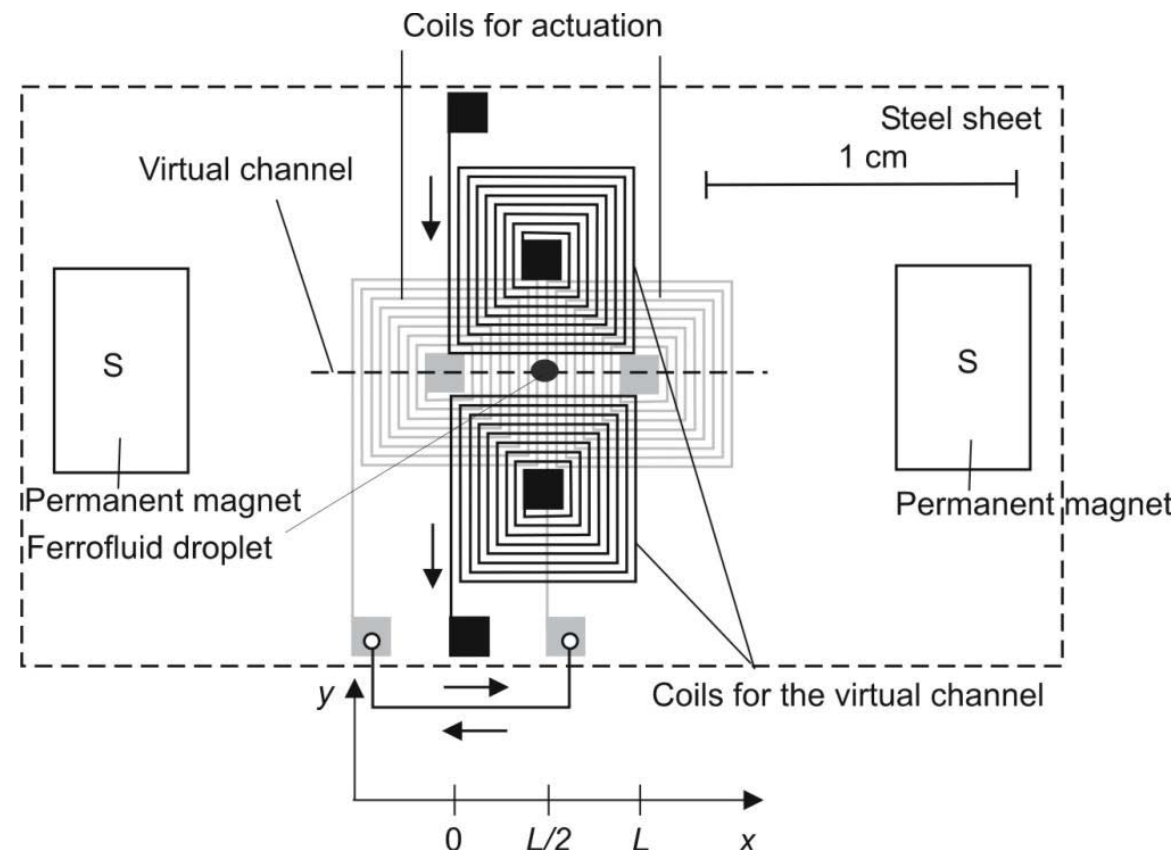

Figure 2-9 Device used for driving the a ferrofluid droplet [100].

As shown in Figure 2-9, complicated arrangement of the permanent magnets and coils were studied and used to achieve tunable droplet actuation. The external magnetic field in this work was generated by a double-sided printed circuit board on a soft magnetic sheet with permanent magnets on both sides. Two planar coils were integrated separately on each side of the printed circuit board. Two coils formed a "virtual channel" to confine the droplet in a single line. The other two coils served as actuating magnetic sources. The reciprocating motion of the ferrofluid droplet could be achieved through reversing the current in the coils. The two permanent magnets together with the magnetic sheet generated a uniform magnetic field for the polarization of suspended particles in the ferrofluid droplet. Nguyen et al. [100-101] carried out experiments to investigate both the deformation and kinematics of a ferrofluid droplet using this device. The effect of different parameters such as droplet size, current in the coils and viscosity of the liquid was discussed. Lehmann et al. [102] 
used a similar device to drive the droplets surrounded by oil in a reservoir. By using a bottom surface with tunable hydrophobic/hydrophilic properties, different forms of manipulation such as splitting, transport, merging and mixing could be achieved. Experimental results were analyzed to examine the relation between the droplet speed and magnetic field to estimate the forces involved.

The above-mentioned configuration avoided external moving parts in the microfluidic device. Fabrication and integration of the system would be flexible and simple. A similar system was built based on a switchable matrix of simple coils on a printed circuit board. The system was successfully used in DNA manipulation [103]. Varying the currents in the coils allowed controlling the magnetic force exerted on the magnetic particles suspended in the droplet. Based on this droplet manipulation system, the detection of enzyme-tagged antibody was proven to be possible, indicating potential applications in bioanalysis [104].

In addition, the magnetic field could be applied in a rotational way. A much more complex actuation scheme could be realized with both radial and tangential stress acting on the magnetic particles. The first experimental work was done by Bacri [105] to observe the droplet response to both the frequency and the magnitude of the applied field. In the experimental work by Shikida [106], both transportation and agitation were carried out through fixed magnets and flat coils on a rotary platform. 


\subsubsection{Magnetically-induced manipulation of liquid in continuous microfluidics}

Continuous microfluidics is widely studied in microfluidic field because of the easier implementation and less sensitive to protein fouling. Numbers of well-developed chemical and biochemical processes can be accomplished with continuous microfluidic devices. The review on MR is not presented as only a few works in microfluidics were reported in this area.

\subsubsection{Magnetophoresis (MP)}

In a magnetic field gradient, magnetic beads moving along the gradient is driven by a magnetic gradient force. This is referred to as magnetophoresis (MP) effect. Magnetic particles consist of an iron oxide core and a polymer shell. The magnetic force $\vec{F}_{\text {mag }}$ acting on magnetic particles carried by a diamagnetic fluid with susceptibility mismatch can be formulated as [107],

$$
\vec{F}_{\text {mag }}=\frac{V_{p}\left(\chi_{p}-\chi_{f}\right)}{\mu_{0}}(\vec{B} \cdot \nabla) \vec{B}
$$

where $V_{p}, \chi_{p}$, and $\chi_{f}$ are volume, susceptibility of the magnetic particles, and the susceptibility of the carrier. Due to the dependence of magnetic force on the mismatch of susceptibility, manipulation can be achieved for magnetic particles in diamagnetic liquid as well as diamagnetic particles in magnetic liquid. The term $(\vec{B} \cdot \nabla) \vec{B}$ is called the magnetic force field. Since both the magnetic field gradient and field strength contribute to the magnitude of the magnetic force, overlapping an external uniform magnetic field with a local nonuniform magnetic field would result in a strong 
magnetic force.

For on-chip magnetic biological manipulation, design of an integrated microfluidic device was proposed with particles magnetized by a uniform magnetic field [108]. By balancing the magnetic force $\vec{F}_{m a g}$ with the Stokes' drag force $\vec{F}_{d}$ in Eq. (2.26), the magnetophoretic velocity $\vec{u}$ can be estimated as [109],

$$
\begin{gathered}
\vec{F}_{d}=3 \pi \eta d_{p} \vec{u} \\
\vec{u}=\frac{d_{p}^{2}\left(\chi_{p}-\chi_{f}\right)(\vec{B} \cdot \nabla) \vec{B}}{18 \mu_{0} \eta}=\frac{1}{\mu_{0}} \zeta(\vec{B} \cdot \nabla) \vec{B}
\end{gathered}
$$

with,

$$
\zeta=\frac{d_{p}^{2}\left(\chi_{p}-\chi_{f}\right)}{18 \eta}
$$

being the "magnetophoretic mobility (MM)" of the particle. With the same magnetic force field, the particle velocity is proportional to square of the particle diameter $d_{p}$. The smaller the particle size, the smaller is the particle velocity.

MP is suitable for separation process as magnetic particles are commonly used in bioanalysis as solid support material for antigens, antibodies, DNA and even cells. With a functionalized coating, targeted biomolecules or cells can be attached and then trapped by applying an external magnetic field.

In conventional magnetic separation microfluidic devices, the tagged material is immobilized while the non-labeled cells are flushed away with the buffer. After switching off the magnetic field, the trapped material is flushed and collected in the subsequent step. This is termed Magnetic Activated Cell Sorting (MACS) [110-111]. Permanent magnets are commonly used in conventional magnetic separators to 
produce spatially non-uniform magnetic fields. The requirement of yoke frames, wires, and holders increases the cost of the device fabrication. In addition, this is a batchwise method which is time consuming since many bioanalytical procedures require a large number of sequential handling processes.

For strongly spatial varied magnetic field, the expression of magnetic force was much more complicated. To obtain a regular dependence of magnetic force on position, Zborowski et al. [112-114] utilized a quadrupole permanent magnetic field for continuous separation of material. Mathematical prediction was presented of the magnetic and viscous forces. The experimental testing of the sorting performance was carried out with a model cell system of human peripheral lymphocytes targeted with commercial monoclonal antibodies and iron-dextran colloid. The measurement of MM by cell tracking velocimetry (CTV) was first reported in the application of red blood cell magnetophoresis [115]. The same group fabricated a high-capacity quadrupole magnetic flow sorter for progenitor cell isolation [116], and separating a breast cancer cell line from human blood [117]. The purities and throughput of the progenitor cell separation were $64-95 \%$ and $10^{7}$ cells/s, respectively. Based on the MM distribution of cell obtained by CTV, theoretical model was developed with experimental verification using breast carcinoma cells (HCC 1954) overexpressing the HER-2/neu gene, and peripheral blood leukocytes (PBLs). High throughput $\left(3.29 \times 10^{5}\right.$ cells $\left./ \mathrm{s}\right)$ separation was performed with high recoveries (0.89) of HCC1954 cells. The similar device was tested for immunomagnetic labeling of CD34+ cell and the isolation from fresh leukocyte fraction of peripheral blood [118]. 


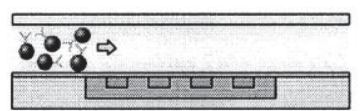

(a)

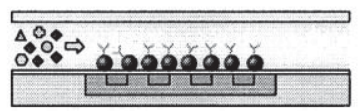

(c)

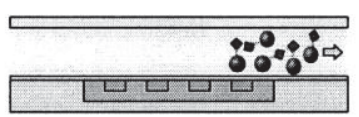

(e)

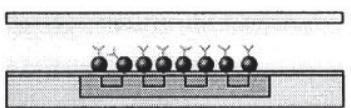

(b)

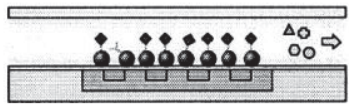

(d)

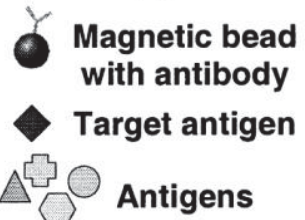

Figure 2-10 Illustration of batchwise filter: (a) Injection of magnetic particles, (b) Trapping of magnetic particles, (c) Samples flowing, (d) Immobilization of target antigen, (e) Release of antigen. [119]

Instead of using a permanent magnet, various design and fabrication of integrated electromagnet was proposed and tested. $\mathrm{NdFeB}$ magnets with small iron tips attached were used in a capillary magnetophoretic device to enhance the magnetic field gradient for trapping of red blood cells [120]. The integrated inductive components were beneficial for design flexibility, compactness, and device feasibility. Ahn et al. [121] reported a micromachined integrated magnetic separator by using meander-type inductors, quadrupole electromagnets. The batchwise technique was also mentioned as a filterless approach for magnetic separation [119]. The magnetic field was generated by an integrated planar electromagnet fabricated with serpentine conductors and semiencapsulated in nickel-iron $(\mathrm{Ni} / \mathrm{Fe})$ permalloy. The separation procedure was illustrated in Figure 2-10. In order to obtain relatively high inductances, spiral-type inductive components were employed for actuation [122]. In this configuration, spiral conductors were positioned on an insulated substrate, a magnetic substrate or between two magnetic films. The inductance could be four to five times higher when an 
electroplated high-permeability $\mathrm{Ni} / \mathrm{Fe}$ magnetic core was added. The central core exhibited the benefits of concentrating flux and completely encapsulating the windings. The similar design and fabrication of spiral electromagnets was employed for magnetic bead separation [123]. For confining flux to achieve low field losses, semiencapsulated Permalloy core was applied with a high permeability.
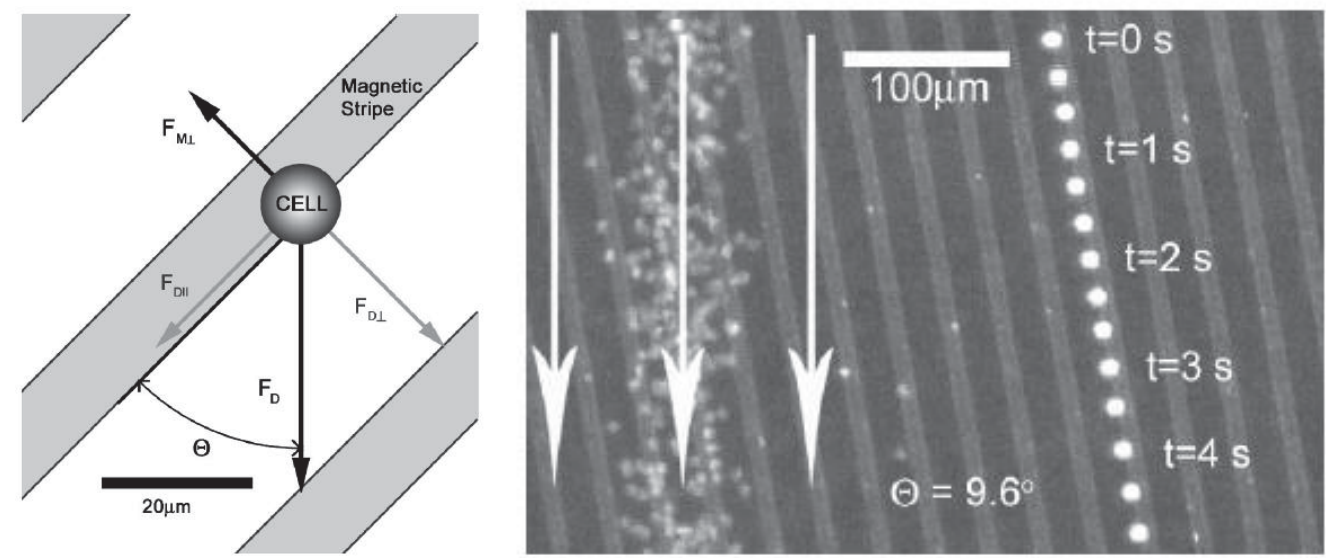

Figure 2-11 Diagram of continuous magnetic separating with ferromagnetic strips fabricated inside the channel: (a) Top view of a magnetically labeled cell over a magnetic stripe; (b) experimental images of red blood cells separation from white blood cells.[124]

In the above-mentioned magnetic separating and sorting devices, magnets were positioned or integrated outside of the microfluidic channels. Inglis et al. [124] demonstrated a continuous magnetic separator with some ferromagnetic strips fabricated inside the channel as shown in Figure 2-11. These ferromagnetic structures provided an array similar to the magnetic field pattern at a given angle to the flow field. Magnetically-tagged particles would deviate to follow the strips once activating the magnetic field. The device was used for separation of magnetic red blood cells from diamagnetic white blood cells. In another work, serpentine wires were used to trap magnetic particles and move them by superimposing an external magnetic field on the 
field from the electric wire [125]. On the inner wall of the microfluidic channels, arrays of Ni posts magnetized by a permanent magnetic field were demonstrated to be capable of trapping magnetic beads passing through [126]. As a result, the separation of magnetic beads was realized from a solution containing both magnetic and diamagnetic beads.

With combining magnetic separation with free-flow fractionation (FFF), the continuous immune magnetic sorter performed with high throughput rate, high purity and good cell viability [127-128]. The separation of magnetic particles from diamagnetic particles were experimentally achieved in a chamber under a fanned out, inhomogeneous magnetic field. As shown in Figure 2-12, Fuh et al. [129-130] utilized magnetic actuation in split flow thin fractionation (SPLITT) for particles separation. The principle was proposed to separate particles with different magnetic susceptibility into different fractions by adjusting the magnetic field strength and the orthogonal flow velocity. The flow system consisted of a buffer and the other stream containing magnetic particles. Under a magnetic force, the magnetic particles shifted away from their stream into the buffer stream. The collection of separated particles was realized by multiple outlets of the working channel. In this device, the complete separation could only be achieved for particles with minimum difference in magnetic susceptibility of $3 \times 10^{-6} \mathrm{cgs}$. The sample recovery rates were higher than $94 \%$. The throughput of the tested device was approximately $3 \mathrm{~g} / \mathrm{h}$. Chronis et al. [131] reported a theoretical investigation of magnetic separator based on continuous hydrodynamic parallel flow in an H-shaped channel. 


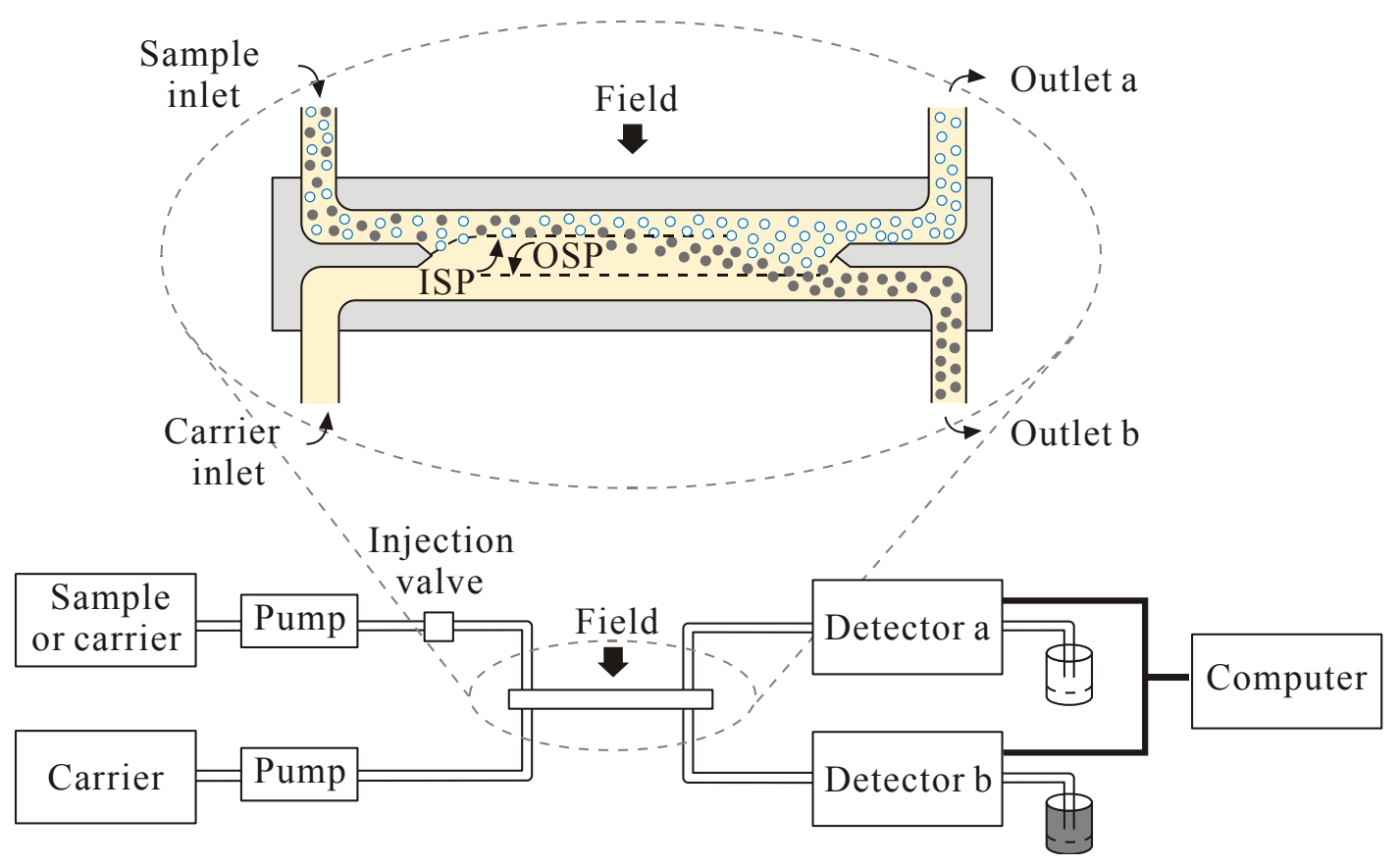

Figure 2-12 Schematic illustration of a magnetic SPLITT separation system. [129]

To overcome the limitation of batchwise method, MP was widely explored for continuous magnetic separation process which was capable of handling large cell volumes, on-line visualization of the separation process, staging and recirculating separated material. The so called free-flow magnetophoresis (FFM) was proposed based on free-flow electrophoresis principle. In a microchannel, the mixture of different magnetic and non-magnetic particles flowed along the channel wall. Nonuniform magnetic field was generated transverse to the laminar flow by placing a micro magnet upon the channel. Different magnetic particles were deflected more or less from their path. The non-magnetic particles remained in the original flow path. The additional space enabled the collection of the separated particles. Two different magnetic sorting purposes could be realized in FFM devices: concentration or depletion of magnetically labeled particles. Concentration meant that the target particles were magnetically labeled and focused under the magnetic force. The 
disadvantage was the requirement for subsequent detaching process. Depletion meant the labeling of all particles except the desired particles.

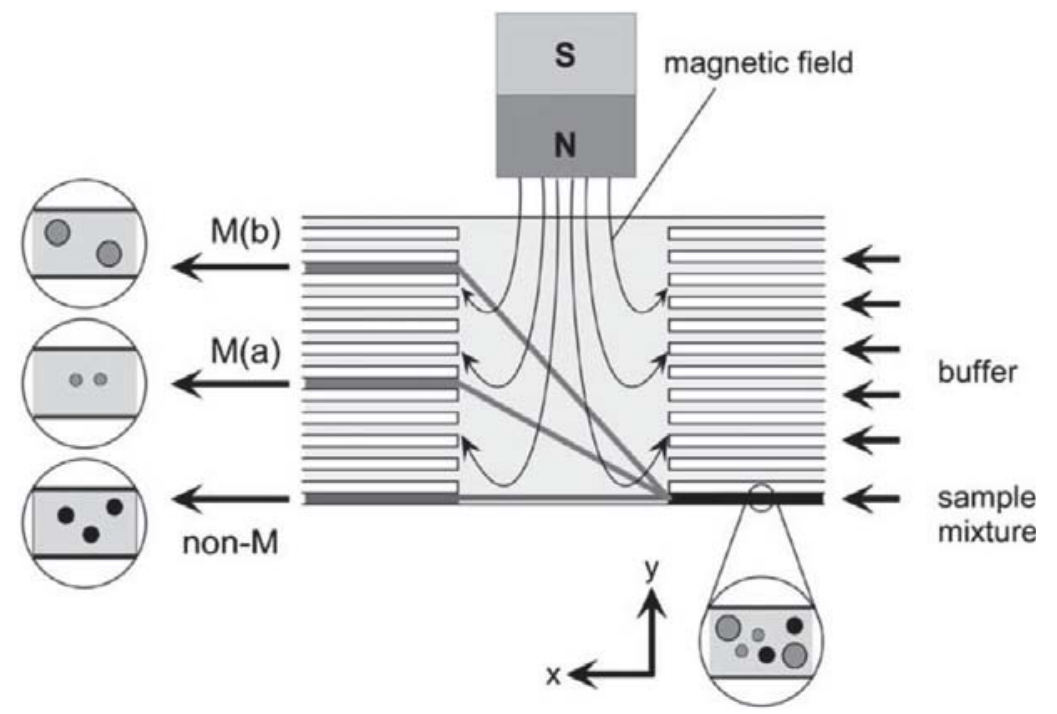

Figure 2-13 Schematic illustration of free-flow MP. [132]

Pamme et al. developed the technique called 'on-chip (FFM)' and demonstrated the feasibility in separating magnetic particles from diamagnetic particles [133], as well as magnetic particles of different sizes [132]. The 4.5- $\mu m$ magnetic particles with larger susceptibility were deflected further from the original flow path than the 2.0- $\mu m$ magnetic particles. Nonmagnetic 6- $\mu m$ beads were not deflected at all. Additionally, agglomerations of magnetic particles were found to be deflected to a larger extent than single magnetic particles. The proof-of-principle manipulation was investigated in terms of the flow rate, the magnetic field strength and the field gradient. The configuration of the working channel was illustrated in Figure 2-13 with multiple inlets and outlets channel for forming a laminar flow. To improve the design, the junctions were tapered for smooth flow through the chamber. The detection of separated particles was realized by the video observation and on-chip laser light 
scattering. The trajectories of particles were predicted by numerical calculation with assuming spherical cells without significant deformation during deflection [134].

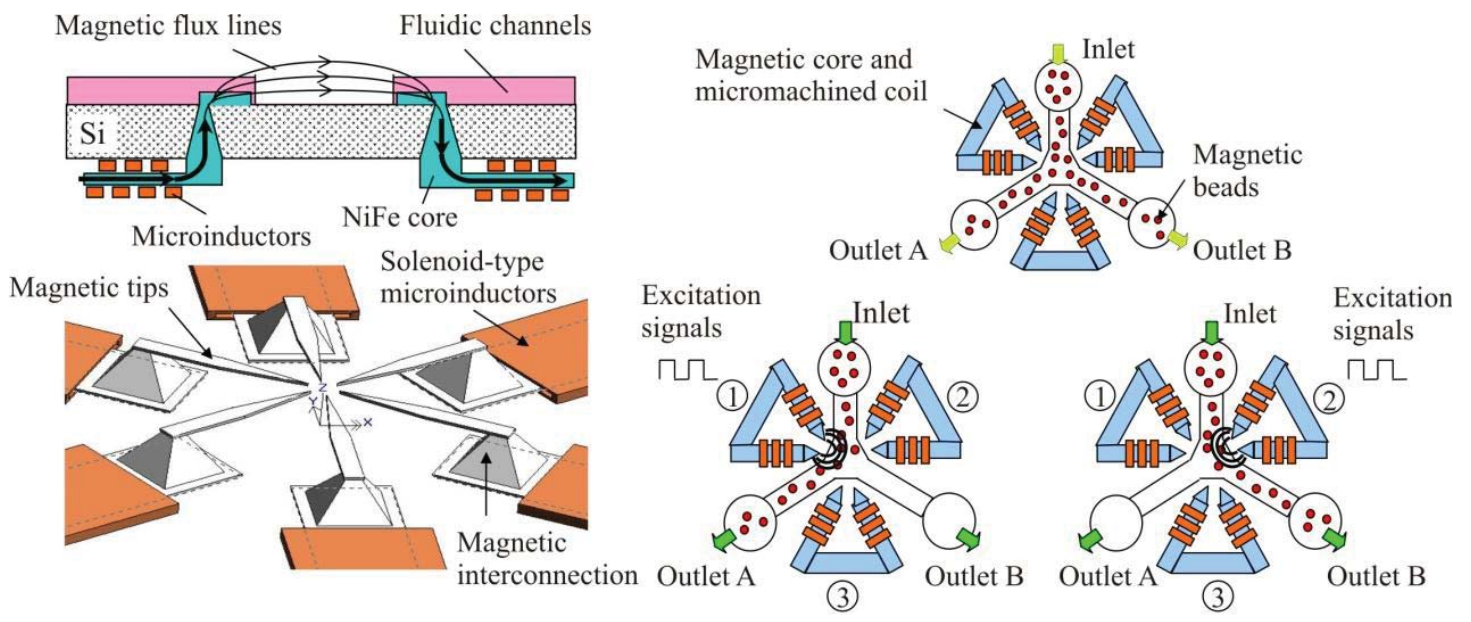

(a)

(b)

Figure 2-14 The on-chip bead separator (a) the configuration of the device; (b) the operating of the separating principle[135]

To increase magnitude of magnetic force, different configurations of magnets were used in continuous magnetic separators. Small, cylindrical, strong, permanent neodymium-iron-boron $(\mathrm{NdFeB})$ magnets were used to generate a magnetic field (mean gradient of $20 \mathrm{mT} / \mathrm{mm}$ ) over the separation chamber [133]. The design and fabrication was also presented for micromachined magnetic tips in a continuous microfluidic separator system as shown in Figure 2-14 [135]. The fully integrated electromagnetic inductors were placed underneath the device with the application of magnetic interconnection technology with through-holes filled with NiFe permalloy material. Since only the magnetic tips were exposed to the working microchannels, the flexibility of the device was greatly improved. Furthermore, the shape of magnetic tips offered a high magnetic field gradient which contributed to the force on magnetic components. In operation, inductors working in pairs drove magnetic particles to the 
corresponding channel with applying a signal.

The magnetic separation principle was applied in the purification of single-walled carbon nanotubes (SWCNTs) from the superparamagnetic iron-catalyst impurities in a microfluidic device without affecting the inherent SWCNT properties [136]. The magnetic field gradient was enhanced by using a saw-tooth nickel microstructure. The highly purified SWCNTs (around 98-99\%) were achieved from the initial SWCNTs with iron content at around $18.14 \%$. In order to increase the sensitivity of the magnetophoretic method, the magnetic mobility distinction was enhanced under a susceptibility gradient of the surroundings generated by diffusion of paramagnetic diethylenetriamine-pentaacetic acid (Gd-DTPA) solution into the core stream of diamagnetic D-glucose solution. The magnetic particles would stay focused at a certain location where the susceptibility of the particles was identical to that of the surrounding. This principle was termed as isomagnetophoresis due to its analogy with isoelectrophoresis.

\subsubsection{Ferrohydrodynamics (FHD)}

Magnetic manipulation and sorting of particles and cells could be realized by using ferrofluid [137-139]. Continuous separation of non-magnetic particles can be achieved in a magnetic surrounding. In the presence of an external magnetic field gradient, the immersed microparticles undergo a magnetic buoyancy force and thus moved away from the magnetic field source. This principle was termed as negative magnetophoresis. 


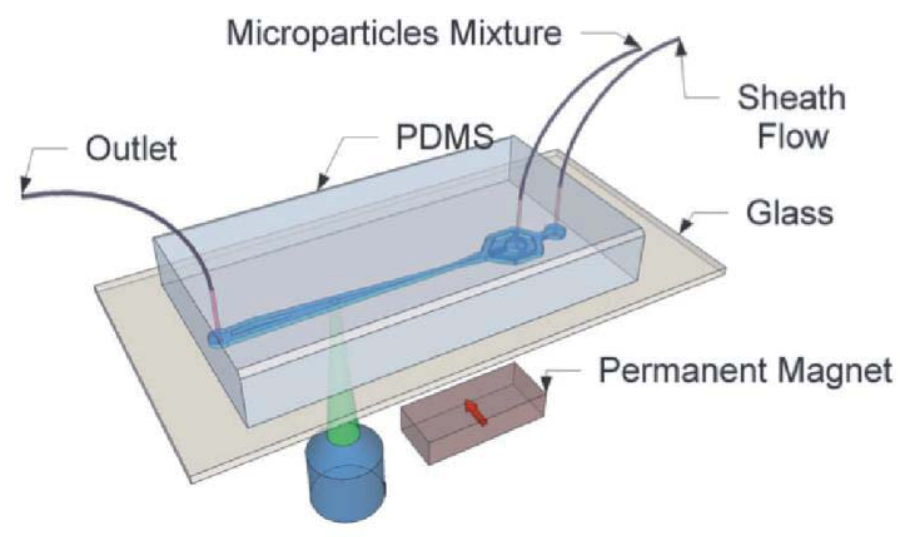

Figure 2-15 The typical experimental device for separation with negative magnetophoresis [140-141].

Gao et al. [142] termed the ferrofluids containing non-magnetic particles as inverse ferrofluids. In the presence of a nonuniform magnetic field, the magnetophoretic force exerting on nonmagnetic particles in inverse ferrofluid was theoretically investigated based on the Ewald-Kornfeld formulation and MaxwellGarnett theory. The typical device for microfluidic separation was shown in Figure 2-15 for negative magnetophoresis principle [140-141]. Non-magnetic particles carried by water-based ferrofluid were delivered and hydrodynamically focused in the microchannel. When passing through the region affected by the magnetic field, shift of non-magnetic particles occur due to the magnetic force under a non-uniform field, Eq. (2.25). The dynamics of the non-magnetic particles could be obtained by balancing the magnetic force with the Stokes drag force.

For focused particles stream, the magnetic force was proportional to the volume of the particles. The larger the particles, the longer were the shifting distance. The sizedependent separation was ensured for non-magnetic particles in a magnetic medium. A 2D numerical simulation demonstrated distinct trajectories for particles with different 
sizes. With ferrofluid flow (1 $\mu \mathrm{L} / \mathrm{min})$ sandwiched by sheath streams $(2.5 \mu \mathrm{L} / \mathrm{min})$, the fabricated device was capable of separating $\sim 10^{5} 9.9 \mu \mathrm{m}$ particles from $\sim 10^{7} 2.1 \mu \mathrm{m}$ particles with efficiency at $100 \%$. For two series of particles with smaller size gap, the separation efficiency of $100 \%$ was also achieved. The theoretical foundation of sizedependent particles separation in ferrofluid was provided with an analytical model on microfluidic transport [143]. Trajectories were obtained for non-magnetic particles in a permanent magnetic field. The model allows the optimization of separation device according to parameters such as the particle size, the ferrofluid type, the magnetic field distribution, the channel size, and the flow rate of liquid. The same group carried out the separation of Escherichia coli and saccharomyces cerevisiae from ferrofluid [144]. The sorting throughput and efficiency were $10^{7}$ cells/h and $100 \%$, respectively. Based on the previous theoretical investigation [143], Liang et al. [145] reported an experimental investigation which agreed well with the theory that takes the "wall effect" into consideration.

Focusing particles into a tight stream was a critical issue in many microfluidic applications, such as flow cytometry and particles sorting. Based on the manipulation of particles in a paramagnetic surrounding, magnetic focusing could also be realized by using ferrofluid. For microchannel with a T-junction structure at the entrance, nonmagnetic particles carried by a diluted ferrofluid were demonstrated to be focused to the center plane confined by a water stream [146]. The magnetic field was generated by a single permanent magnet. Zhu et al. [147] also incorporated a pair of NdFeB magnets to the microfluidic device for producing the required magnetic fields for 
focusing. Focusing of 4.8, 5.8, and $7.3 \mu \mathrm{m}$ microparticles was achieved with various flow rates in a microfluidic channel. In addition, a 3D analytical model was developed for predicting trajectories of microparticles with high efficiency and accuracy.

Furthermore, negative-magnetophoresis with ferrofluid was demonstrated to be suitable for assembling of particles [137]. The basic principle was introduced for particle transport and assembly. Nonmagnetic particles tend to assemble at location of minimum magnetic field strength, which indicates energy minima. The substrate was patterned with an array of traps for separating energy minima by potential energy barriers. Rotating magnetic field was required for particle assembly to adjust the orientation of the field with respect to trap magnetization. Solenoid coils were adopted and arranged to ensure control in three orthogonal directions. Large single-layer arrays of micrometer-sized particles were demonstrated to be able to assemble onto magnetic traps surrounded by ferrofluid with low concentration. Particle transport required the trap magnetization to be maintained in a constant direction. Thin ferromagnetic film patterned into isolated islands was used for particles assembly under a permanent magnetic field [148]. Yellen [149] demonstrated the self-assembly of multiple different types of colloidal objects in close proximity on the same substrate by using thin microfabricated magnets covered with microwells. The assembly and manipulation of nonmagnetic latex particles $(100-300 \mathrm{~nm})$ were demonstrated with submicron Co islands magnetized by a uniform, static, or rotating magnetic field [150]. The rotation or shift of the external magnetic field would give rise to the corresponding change in potential-energy minima. The movement of the particles 
could be determined when the depths of the potential-energy wells dominated over the thermal Brownian motion. Feinstein and Prentiss [151] demonstrated a 3D selfassembly of structures by using the differential pressure resulted by a ferrofluid interacting with a magnetic field gradient. This method maintained the particles aggregates without attaching to a plane solid surface. Friedman and Yellen [152] presented a review on the basic underlying principle and models for separation, manipulation and assembly of solid non-magnetic phase using an external magnetic field.

Magnetic manipulation of convective mass transport also offered more productive handling of many tasks of biological, chemical, medical and environmental research. The control of convective mass transport was critical in lab-on-a-chip devices involving sample separation, mixing and further analysis. The redistribution of reagents could be achieved by diffusion-mediated effect in laminar flow or stirring effect of fluid flow within micro channels. Normally, diffusion-mediated effect could be achieved by propelling liquids circulation to realize the increase of interfacial area. However, propelling and stirring of fluids was challenging in microchannels. For a rapid and efficient mass transport process, various applications of ferrofluid were reported for magnetically-induced driven of magnetic components in microfluidic devices.

Mixing was achieved by micro stirrers[153-154] and magnetohydrodynamic instability $[37,43]$. The capability of magnetic manipulation using an external field led to additional applications such as tagging target entities to the magnetic particles in a 
bio separator. The feasibility of controlling magnetic particles using an external magnetic field provided the option of wireless manipulation, which led to the possibility of drug delivery using magnetic particles.

Chaotic motion of magnetic particles could be induced to increase the interfacial area of fluids and consequently their mixing efficiency. For instance, Suzuki and Ho [155] numerically investigated mixing induced by magnetic beads in a serpentine channel. The chaotic motion of magnetic beads was realized by applying a locally time-varying magnetic force. The magnetic force was generated by embedding straight conductors driven by a periodic current. A similar numerical study was then carried out by Zolgharni et al. [156] using straight channel with embedded serpentine conductors. Taking dipole interactions into account, the chain formation of particles were considered in the simulation. The resulting mixing efficiency was characterized by the concentration distribution and the chaotic advection of magnetic particles. Rong et al. [157] proposed an active micromixer by combining rotational and vibrational force on magnetic beads. The sequential excitation of pairs of electromagnetic microtips led to a rotating magnetic field. To overcome the cost and complexity of the electromagnets, Grumann et al. [158] used the lab-on-a-disk concept with a permanent magnet to improve the mixing process.

Micromixers utilizing ferrofluid were realized and reported previously. Mao and Koser [159] reported mixing of ferrofluid with a fluorescein buffer by using ferrohydrodynamics. Berthier and Ricoul [160] investigated the ferrohydrodynamic instability in the vicinity of the magnet. Both experimental and numerical results 
showed that the instability of ferrofluid would result in mixing in a microchannel. The instabilities of ferrofluid were caused by sudden velocity variation of the flow passing by a permanent magnet. Recently, Wen et al. [161-162] reported the experimental and numerical investigation of an active micromixer based on fingering instability. In this work, ferrofluid and Rhodamine B solution were mixed. The fingering structures appeared at the interface under an alternate magnetic field which was generated by an electromagnet driven by an AC current. With a magnetic field flux density larger than 29.2 Oe and a frequency ranging from 45 to $300 \mathrm{~Hz}$, the mixing efficiency of $95 \%$ could be achieved within 2 seconds.

Extensive efforts were dedicated to study the transport phenomena of magnetic colloids in a magnetic field. Most of the studies focused on magnetic particles in a nonuniform field. The magnetic force was one of the parameters that were affected by the field gradient. In addition to the magnetic volume force in the Navier-Stokes equation, the motion of particles was also affected by the magnetic force acting on each particle. The volume concentration of the particles was governed by a driftdiffusion equation. Blums [163] reported a numerical study on the mass transport of ferrosuspensions and ferrofluids. In the case of micromixing driven by magnetic particles, a numerical simulation was carried out and agreed well with experimental results [164]. The 3D problem was solved by using Spray module of the commercial software CFD-ACE+. For the similar purpose of magnetically-induced mixing, Finite Volume Method (FVM) was used by Le et al. [165] for solving the flow and concentration field with an implicit scheme. A FVM based code was also developed 
and utilized for investigating the effect of a magnetic field on the concentration field of magnetic nanoparticles [166]. In a recent work, the drift-diffusion equation was numerically solved while the magnetic field and force were obtained from an analytical model [167]. A microseparator based on forces induced by magnetic particles was proposed and numerically investigated [168]. Chaotic mixing of magnetic particles inside the microfluidic separator was numerically studied using the commercial multiphysics finite element package Comsol [156]. In addition, there were reports on simulation of mass transport in ferrofluids including the effect of thermomagnetic convection [169-171].

In two-phase continuous microfuidic devices, ferrofluid droplets were formed inside the channel and used to serve as a piston for driven of the other immiscible phase. The principle was presented by Hatch et al. [172] for the magnetic pumping using magnetically-propelled plugs of ferrofluid immiscible with the surrounding fluid to be driven in a circular channel. The closed-loop channel configuration was preferred for continuous process. The ferrofluidic plugs also served as valves held in place by a stationary magnet, which maintained the pressure without extra power consumption. The flow rate was programmable by controlling channel dimensions or the velocity of the moving permanent magnet. Nguyen and Chai [173] demonstrated a stepper micropump by using a series of solenoids magnets to drive ferrofluid plugs inside a closed loop. The actuation force was controlled by adjusting the applied voltage or current. 
Magnetically-induced pumping was applied in rapid continuous-flow (CF) PCR devices. Planar magnetic coils were used to drive a small ferrofluidic plug in a closeloop circular ferrofluid driven microchip [174-176]. Droplet-based PCR was also developed and realized using a magnetic transportation system [177]. The high speed PCR was realized by the combination of magnetic reciprocative movement with thermal cycling. Multi-task PCR was also carried out without cross contamination between the droplets.

In conventional straight microchannels, Andò et al. [178] developed and tested the pump with injecting three ferrofluid droplets into the glass pipe filled with DI water. Two of the droplets worked as valves, while one served as a piston. External coils were used to generate force on each ferrofluid droplet. The miniaturized device was fabricated and tested for pumping of immiscible fluid with synchronizing the actuation of the three plugs [179]. The same group explored the pump with a single ferrofluid droplet injected into the pipe [180]. The ferrofluid droplet was fixed by a sheet of paper-magnet placed in the bottom of the pipe. The paper-magnet could also help to flatten the ferrofluid droplet at the bottom of the channel, which allowed the flow of DI water. The electromagnetic system propelled the peristaltic motion of the plugs, which induced the transport of the immiscible surrounding. The similar concept was reported by Hartshorne et al. [181] of controlling three separate ferrofluid droplets in a glass microchannel. The pump of water and air was achieved by synchronizing the actuation of all the pistons. The efficiency for air pumping was quite low (cycle time of about $30 \mathrm{~min}$ ). The preliminary achievements were ferrofluid valves on chip to 
alleviate the problems such as drying out of reagents, leakage at intersections, and interference with electrophoretic methods [182]. Yamahata et al. [183] used a ferrofluid plug in a plastic microchannel for pumping. The periodic motion of the ferrofluid plug was actuated by an external $\mathrm{NdFeB}$ permanent magnet and converted into a pulsed quasi-continuous flow by two serial check-valves formed by integrating two soft silicone membranes. The flow rates and back pressures of the pumped water could be achieved up to $30 \mu \mathrm{L} / \mathrm{min}$ and 25 mbar, respectively.

In a trapezoidal silicon microchannel, the thermally mediated viscosity property of ferrofluid was utilized for more precise controlling of the response time and the net unidirectional flow [184]. The pump mechanism of using ferrofluid was applied for liquid sampling as well which is critical in many biological procedures. Miller and Resler [185] proposed the pumping of a discrete volume of ferrofluid in a small tube under a magnetic field gradient generated by a series of electromagnets. This principle was then utilized for ferrofluid-magnetic pipette [186]. In order to sample small amount of liquid, the array of electromagnets were used to actuate the change of position for ferrofluid. The position shift of $0.1-0.5 \mathrm{~mm}$ could be achieved by adjusting the field gradient. For precise actuating and fast sampling in submicroliter range, permanent magnets were used instead of electromagnet. Permanent magnets was advantageous in no heat generation, fast response, high accuracy resulted by the programmable speed and step angle of the stepping motor [187]. 


\section{CHAPTER 3}

\section{DEFORMATION OF A FERROFLUID DROPLET IN A UNIFORM MAGNETIC FIELD}

In this chapter, the deformation of a sessile ferrofluid droplet on a hydrophobic surface under the effect of an applied magnetic field is studied, both experimentally and numerically. In the experiment, the uniform magnetic field is generated by an electromagnet. The ferrofluid droplet is inserted into the air gap between the coils. The magnetic field is oriented parallel to the substrate surface. A sample container filled with mineral oil is required for the experiment. The mineral oil prevents liquid evaporation and makes the surface apparently superhydrophobic due to the thin film of oil between the substrate and the ferrofluid droplet. The geometry of the droplet is recorded while the flux density of the applied magnetic field is varied with the applied current. A numerical model with nonlinear magnetization is developed and implemented to study the droplet shape under an applied uniform magnetic field coupled with interfacial tension and gravitational forces. The Finite Volume Method (FVM) is used to solve the governing equations. Interface tracking is achieved by the level-set method. The experimental results are compared with the calculations from the numerical modeling. 


\subsection{Experimental Description}

\subsubsection{Experimental Setup}

Figure 3-1 shows the schematic setup of the experiments presented in this chapter. A ferrofluid droplet is dispensed on a hydrophobic glass substrate. The hydrophobic glass substrate together with the droplet is put in a container filled with mineral oil. This container is inserted into the air gap of an electromagnet. In order to take images of the droplet, a CCD camera is mounted horizontally to record the side view of the ferrofluid droplet.

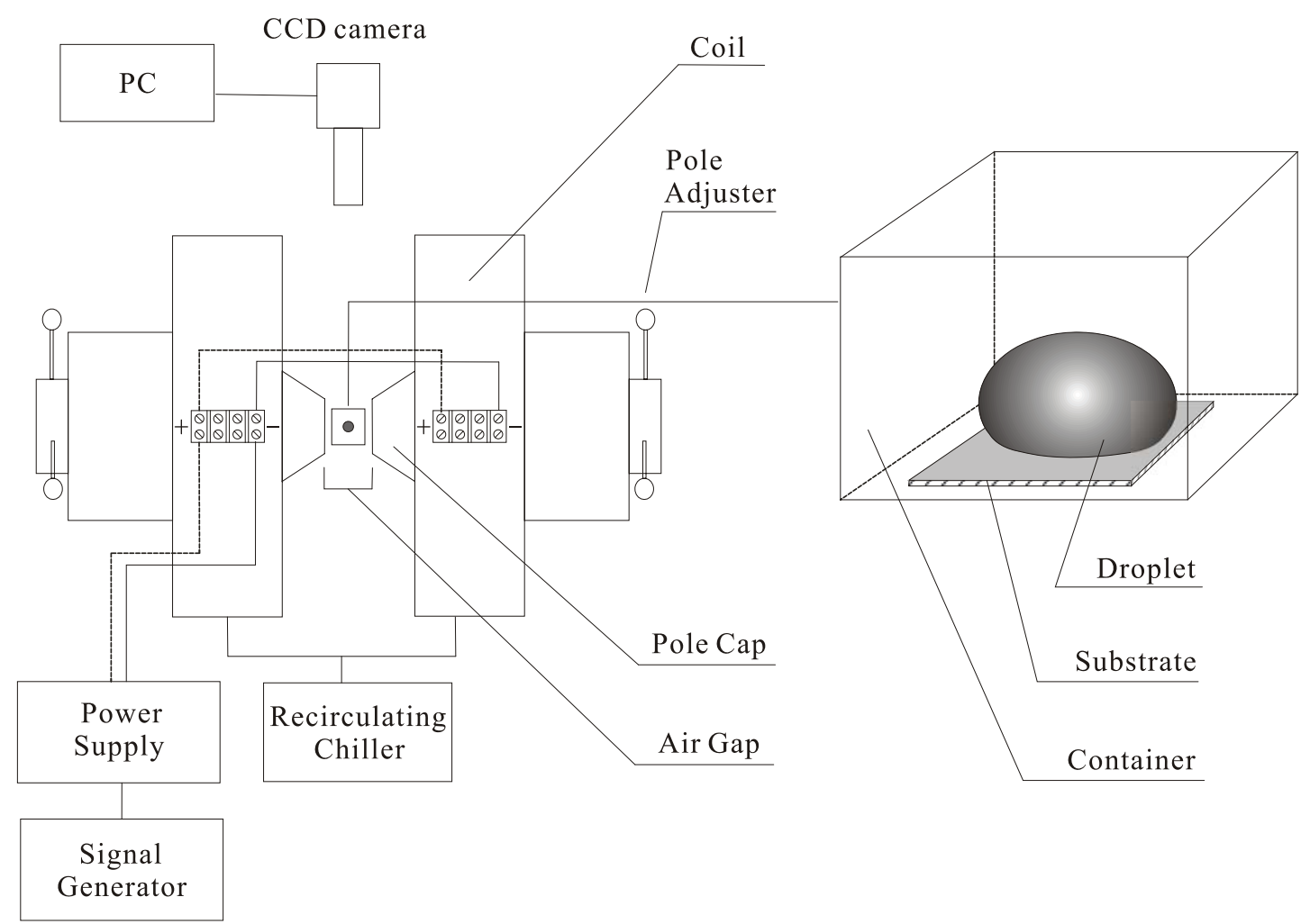

Figure 3-1: Experimental setup and imaging system. 


\subsubsection{Materials and Methods}

In the experiment, the planar substrate was prepared on a Pyrex glass wafer. In order to obtain the hydrophobic properties, we treated the surface using the method reported by Long et al [71]. The glass wafer was first rinsed in Piranha solution to remove any organic matter on the surface. To improve the adhesion of the Teflon coating, a solution of 1 wt \% $1 H, 1 H, 2 H, 2 H$-perfluorodecyltriethoxysilane (Sigma Aldrich) was prepared. The base solution consisted of isopropanol with $5 \mathrm{wt} \%$ water and a drop of $0.1 \mathrm{M} \mathrm{HCl}$. The clean glass wafer was subsequently immersed in this solution for $30 \mathrm{~s}$ and dried at $110^{\circ} \mathrm{C}$ for $15 \mathrm{~min}$. Teflon solution (DuPont AF1600, $1 \mathrm{wt} \%$ solution in Fluorinert) was spin-coated on the fluorosilane-treated glass wafer to form a thin layer of about $200 \mathrm{~nm}$ Teflon. The coated glass wafer was then baked at $110^{\circ} \mathrm{C}$ for $10 \mathrm{~min}$, at $165{ }^{\circ} \mathrm{C}$ for $5 \mathrm{~min}$, and finally at $330^{\circ} \mathrm{C}$ for $15 \mathrm{~min}$. The glass wafer was subsequently diced into $3 \mathrm{~cm} \times 3 \mathrm{~cm}$ square pieces, ready for the experiments. The quality of Teflon AF coating was examined with liquid wettability investigation on the surface. The calibration was obtained by using Theta, an optical tensiometer for accurate measurement on static and dynamic contact angle. The calibration was carried out with DI water dispensed on the Teflon coated glass substrate surrounded by air. At first, the repeatability of the calibration procedure was obtained by analyzing the volume of the droplet. Ten droplets with the same size were dispensed randomly on the surface. The volume, left contact angle and right contact angle of the water droplets were presented in Table 3-1. The repeatability was calculated to be $4.3 \%$. It was concluded that the experiment was repeatable. Then the uniformity of the Teflon 
coating was analyzed by the relative standard deviation of the left contact angle $(1.7 \%)$ and right contact angle $(2.1 \%)$. The Teflon was uniformly coated on the glass substrate.

Table 3-1 Volume and contact angle of droplets in calibration process.

\begin{tabular}{ccc}
\hline droplet volume $(\mu \mathrm{L})$ & left contact angle $\left(^{\circ}\right)$ & right contact angle $\left(^{\circ}\right)$ \\
\hline 0.9578 & 82.38 & 83.82 \\
0.9592 & 84.66 & 83.59 \\
0.9950 & 81.64 & 82.37 \\
0.9350 & 85.19 & 83.97 \\
1.0081 & 82.30 & 78.81 \\
1.0196 & 83.39 & 82.97 \\
1.0170 & 81.18 & 81.39 \\
0.9024 & 82.19 & 80.97 \\
1.0146 & 84.41 & 84.23 \\
0.9350 & 81.71 & 81.54
\end{tabular}

The sample container was made of polymethylmethacrylate (PMMA). The size of the container was determined by the available slot between the two poles of the electromagnet. The PMMA holder was machined by laser cutting. The container was assembled by gluing pieces of PMMA together using chloroform. The pretreated planar surface was placed at the bottom of the container. The droplet was surrounded by mineral oil to improve the hydrophobic property of the contact and to prevent evaporation. Water based ferrofluid (EMG707, Ferrotec, USA) was used with 1.8-vol\% 10-nm $\mathrm{Fe}_{3} \mathrm{O}_{4}$ nanoparticles. The viscosity of the ferrofluid at $27^{\circ} \mathrm{C}$ is $\eta_{F F}=5 \mathrm{mPa} \cdot \mathrm{s}$. The initial susceptibility is $\chi_{0}=0.36$. The surface tension of the ferrofluid and air is $50 \mathrm{mN} / \mathrm{m}$. The density is $\rho_{F F}=1.1 \times 10^{3} \mathrm{~kg} / \mathrm{m}^{3}$ at $25^{\circ} \mathrm{C}$. The density of mineral oil (M5904 Sigma) at $25^{\circ} \mathrm{C}$ is $\rho_{o}=0.84 \times 10^{3} \mathrm{~kg} / \mathrm{m}^{3}$. 
A micropipette (Finnpipette, Thermo Scientific, USA) was used for dispensing the ferrofluid droplet. This micropipette was capable of accurately controlling liquid volumes ranging from $0.5 \mu \mathrm{L}$ to $10 \mu \mathrm{L}$.

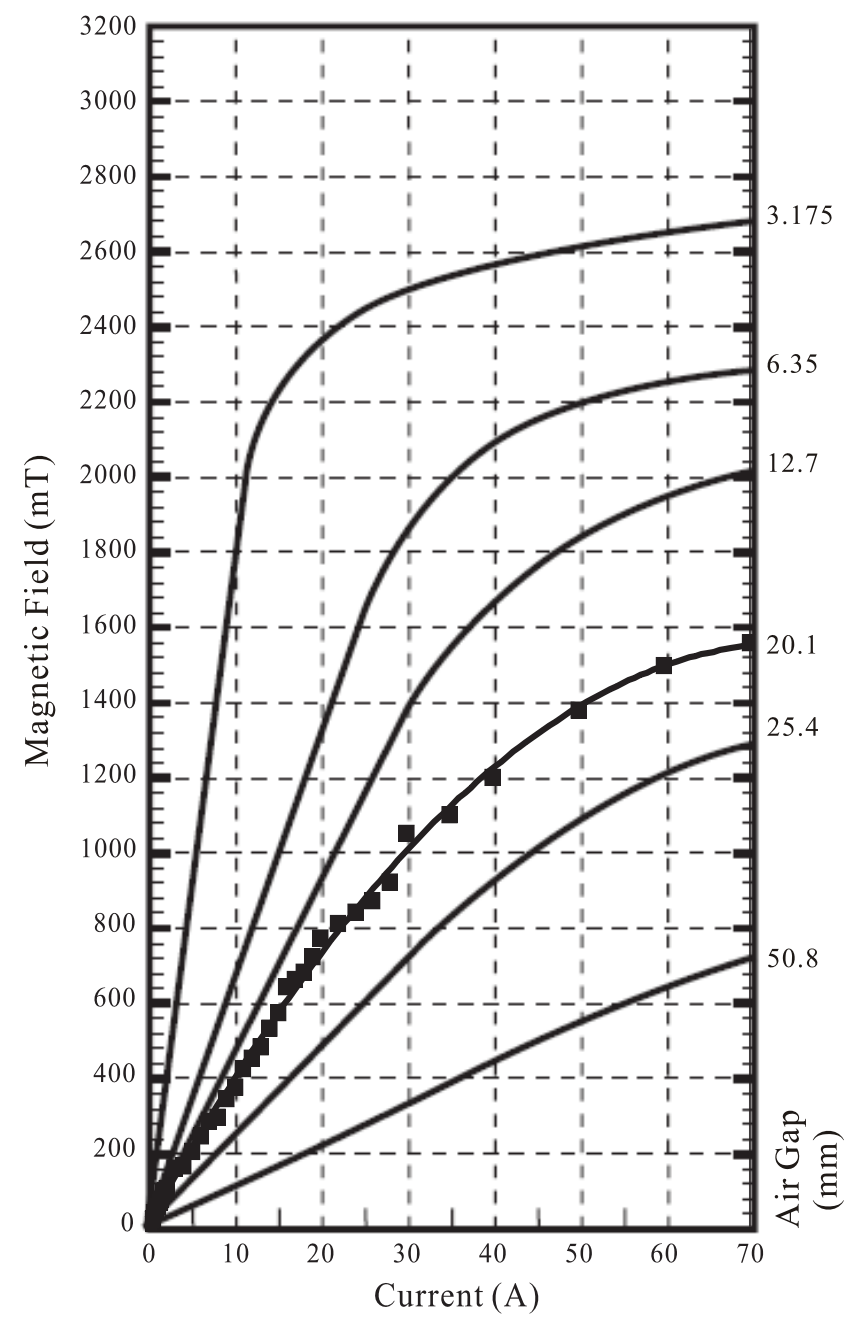

Figure 3-2: Magnetic flux density versus applied current calibration curves of the electromagnet used in the experiments. The data points were measured using a gaussmeter.

A continuous variable gap electromagnet (EM4 Series, Lake Shore, USA) generated a uniform magnetic field for the experiments. Adjusting the variable air gap between the poles can tune the range of the field strength. Once the gap was fixed, the field was controlled by the current input ( $0 \sim 70 \mathrm{~A})$. Good alignment of the tapered pole caps ensured the stability and the uniformity of the magnetic field. Due to the large 
amount of heat generated in the coil, a recirculating chiller (NESLAB Merlin M100, Cole-Parmer, USA) was used for cooling. For a specific value of the field strength, one can refer to the chart given in the manufacturer's manual sheet and the calibration using a gaussmeter (Model 410, Lake Shore, USA). In our experiment, the air gap was fixed at $20.1 \mathrm{~mm}$. For this air gap, a maximum flux density of approximately $1.55 \mathrm{~T}$ could be achieved with a current of 70 A. Figure 3-2 shows the measured data denoted as the square dots, which agree well with the calibration curves provided by the manufacturer.

A CCD camera (Pulnix, progressive scan camera, JAI Inc., Japan) was used to capture the images of the ferrofluid droplets for further processing. A scale was inserted and imaged as a reference for the calibration of the pixel values. A customized MATLAB program processed the images and evaluated the shape of the ferrofluid droplets. The image files were evaluated to extract parameters such as the width and the height of the droplet.

\subsubsection{Stress Balance at Interface and Magnetization}

For a better physical understanding on droplet deformation, the stress balance at the liquid/liquid interface is illustrated based on the ferrohydrodynamic (FHD) Bernoulli equation. Furthermore, the magnetization of the ferrofluid is discussed to facilitate the experimental results analysis. When a ferrofluid droplet is surrounded by a nonmagnetizable medium, the magnetic field induces a mismatch of the normal stress balance at the interface of the two liquids. This stress mismatch drives the droplet to 
elongate along the magnetic field direction. Figure 3-3(a) illustrates the balance of the forces at the interface. The FHD Bernoulli equation can be used to relate the pressures of a gravitational flattened droplet in a magnetic field. Considering a sessile ferrofluid droplet formed by an inviscid, isothermal and incompressible ferrofluid, the FHD Bernoulli equation is written as [80],

$$
p^{*}+\Delta \rho g z-p_{m}=\text { Const }
$$

with the boundary condition,

$$
p^{*}+p_{n}=p_{0}+p_{c}
$$

In Eq. (3.1), $z$ is the elevation of the point above a reference plane; $\Delta \rho$ is the density difference between the ferrofluid and the surrounding fluid; $p_{m}=\mu_{0} \int_{0}^{H} \vec{M} \cdot d \vec{H}$ is the fluid-magnetic pressure with $\vec{H}$ and $\vec{M}$ indicating the applied field strength and the corresponding magnetization parallel to the applied magnetic field; $p_{n}=\mu_{0} \vec{M}_{n}^{2} / 2$ is the magnetic normal pressure with $\vec{M}_{n}$ and $\mu_{0}=4 \pi \times 10^{-7} N \cdot A^{-2}$ representing the normal component of the magnetization and the permeability of vacuum; $p_{c}=2 R \sigma$ is the capillary pressure with $\sigma$ and $R$ indicating the surface tension and the radius of curvature; $p_{0}$ represents the pressure in the nonmagnetic fluid; $p^{*}=p(\rho, T)+p_{m}$ is defined as the composite pressure with $p=p(\rho, T)$ representing the thermodynamic pressure in the magnetizable fluid before polarization. 
(a) Nonmagnetizable liquid

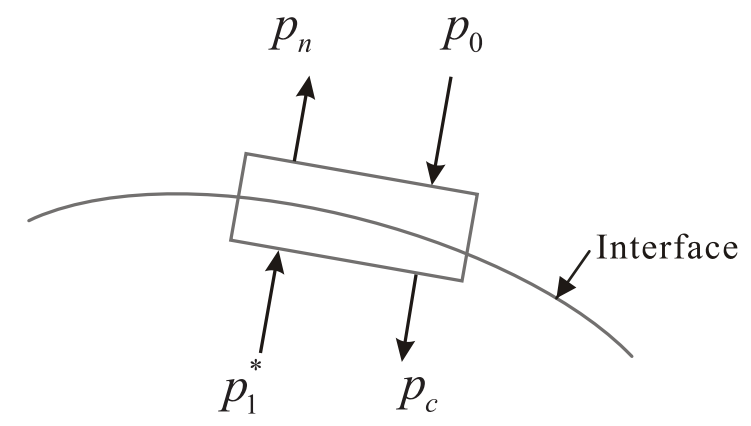

Magnetizable liquid (b) Nonmagnetizable liquid

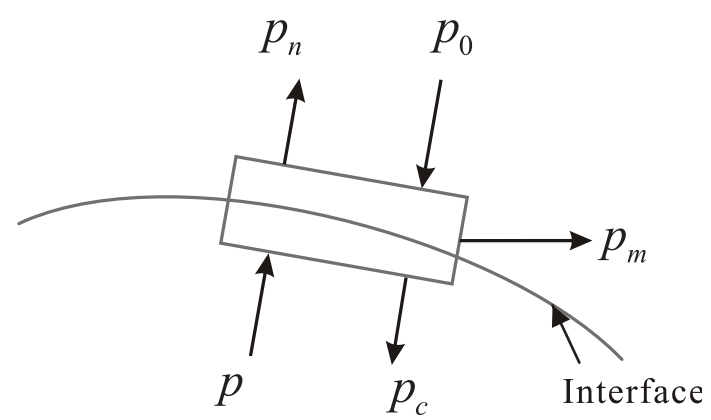

Magnetizable liquid

Figure 3-3: Force balance in a multiphase system with the magnetizable liquid and the nonmagnetizable liquid (a) General condition; (b) Horizontal uniform magnetic field.

At the interface of a nonmagnetizable liquid and an incompressible nonlinear magnetizable liquid, the magnetic surface force density $t_{n n}$ can be expressed as [72],

$$
t_{n n}=p_{m}+p_{n}=\mu_{0} \int_{0}^{H} \vec{M} d \vec{H}+\frac{\mu_{0}}{2} \vec{M}_{n}^{2}
$$

The surface force density directs from the magnetized to the non-magnetized liquid. Eq. (3.3) indicates that the magnetic field strength and the corresponding magnetization of the ferrofluid determine the surface force density, and consequently the elongation of the droplet. In our investigation with a horizontal uniform magnetic field, the stress on the interface is given in Figure 3-3(b).

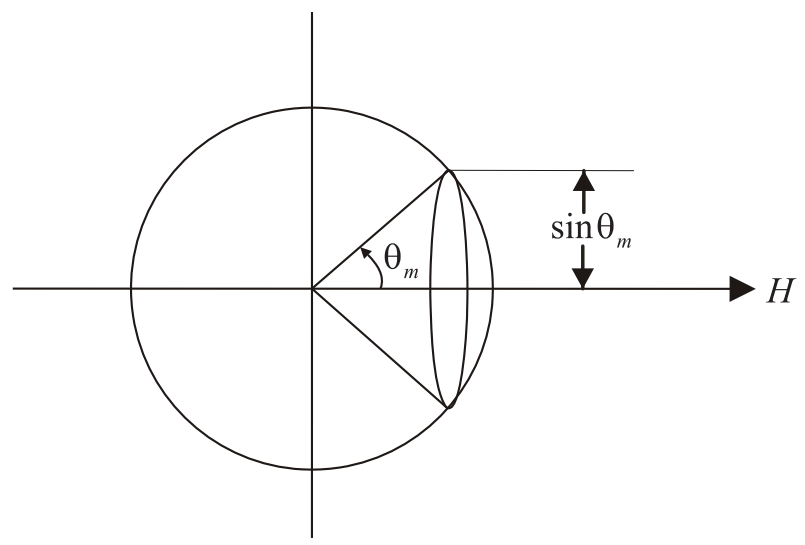

Figure 3-4 Orientational configuration for an independent particle [72] 


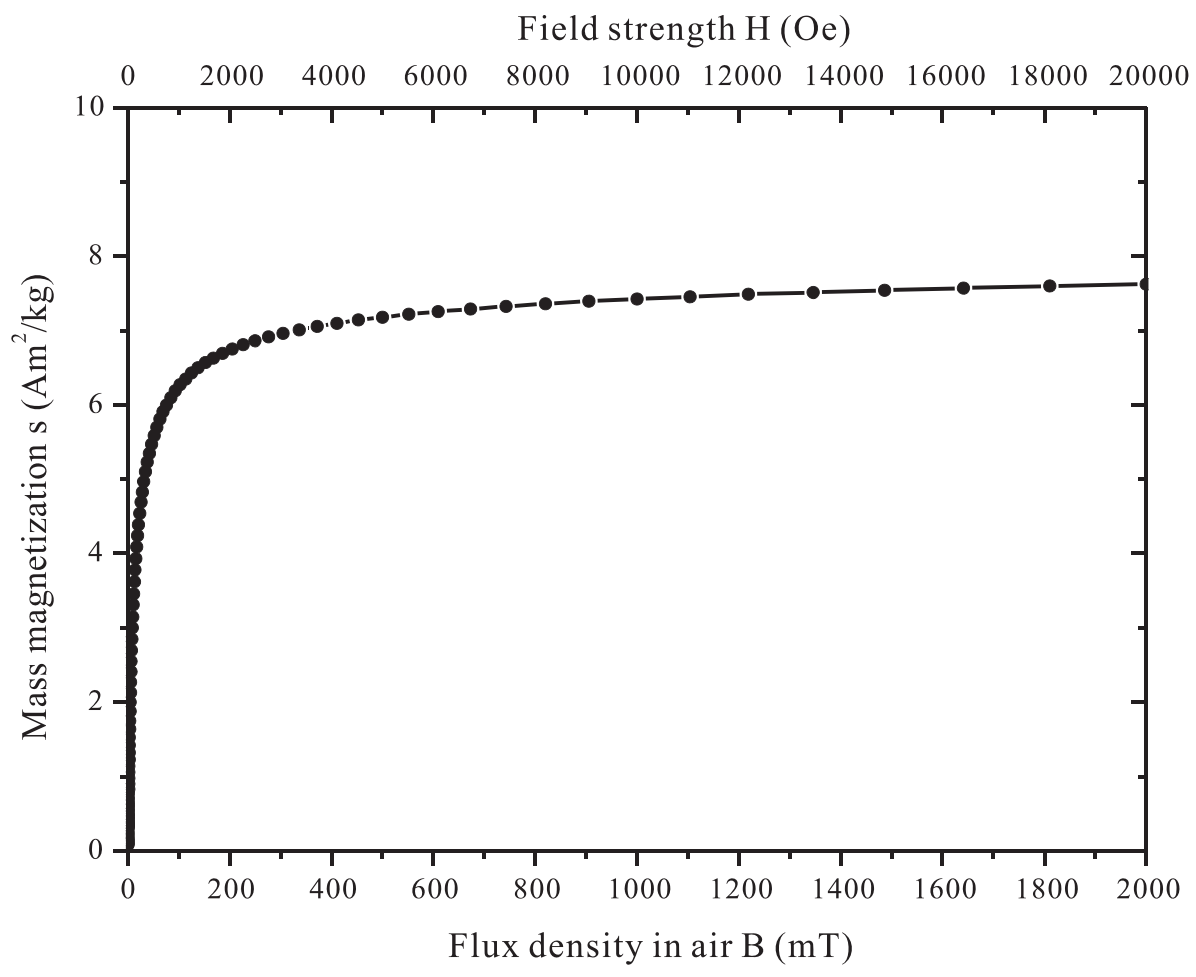

Figure 3-5: Magnetization curves of the ferrofluid used in our experiments (EMG707, Ferrotec, at $285 \mathrm{~K})$.

The magnetic particles of a colloidal ferrofluid are dispersed in the paramagnetic fluid. However, these particles carry magnetic moments. In the absence of an external magnetic field, dipole directions are random. The magnetic particles are polarized by orienting their dipole moments in the direction of the applied field. During the magnetization process, the angle between dipole moment and field direction $\theta_{m}$ (Figure 3-4) becomes smaller as magnetic flux density increases. At a given value, the magnetic flux density is so high that the dipole moments may be completely aligned and saturation magnetization is achieved. The magnetization $\vec{M}$ of a ferrofluid is a vector with the same direction as the applied field. The magnitude of magnetization follows the Langevin law as a function of the field strength $H$ [188],

$$
M=M_{s} L\left(\frac{3 \chi_{0}}{M_{s}} H\right)=M_{s}\left(\operatorname{coth}\left(\frac{3 \chi_{0}}{M_{s}} H\right)-\frac{1}{\left(\left(3 \chi_{0} / M_{s}\right) H\right)}\right)
$$


where $M_{s}$ is the saturation magnetization, and $\chi_{0}$ is the initial value of magnetic susceptibility. The magnetization data in Figure 3-5 was obtained from the manufacturer's data sheet. The magnetization $M$ is calculated from the depicted mass magnetization $s$ as $M=s \rho_{F F}$, where $\rho_{F F}$ is the density of the ferrofluid. Saturation magnetization of the ferrofluid is reached if the magnetic flux density in air is about $150 \mathrm{mT}$.

\subsubsection{Experimental Results and Discussion}
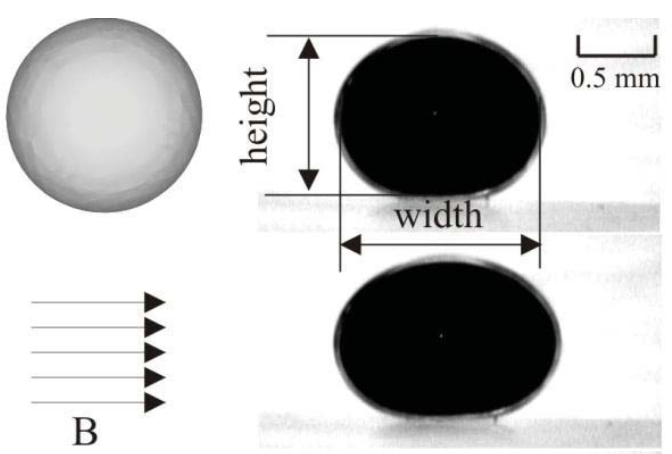

$\mathrm{B}=0 \mathrm{mT}$

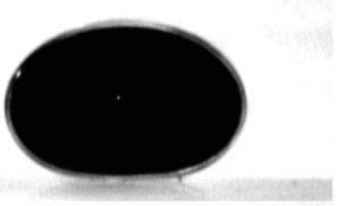

$\mathrm{B}=100 \mathrm{mT}$

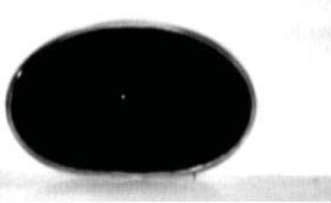

$\mathrm{B}=200 \mathrm{mT}$
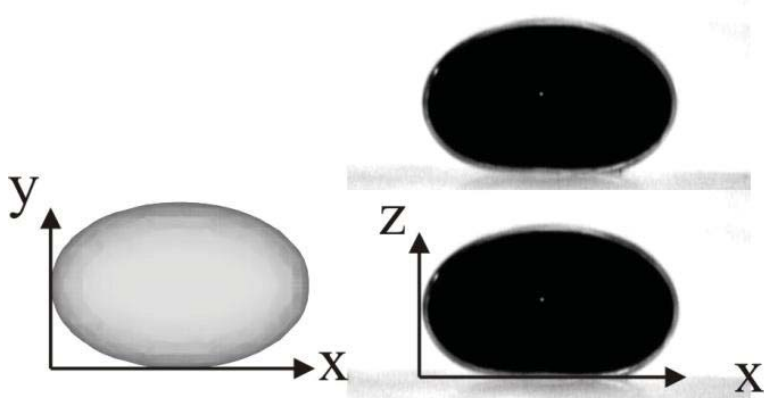

$\mathrm{B}=290 \mathrm{mT}$

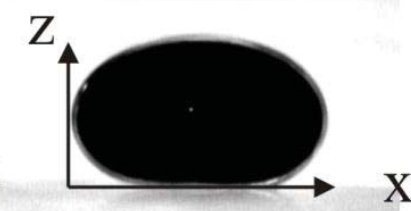

$\mathrm{B}=370 \mathrm{mT}$

Figure 3-6: Deformation of a sessile ferrofluid droplet under different magnetic flux densities B $(V=1.3 \mu \mathrm{L})$. 
The effect of uniform magnetic flux density on the shape of a sessile ferrofluid droplet on a superhydrophobic surface is first investigated. Figure 3-6 shows the typical shapes of a sessile ferrofluid droplet under different magnetic flux densities. The droplet is elongated along the direction of the field. In this experiment, the magnetic field is applied along the $\mathrm{x}$ direction. That is why the droplet will elongate symmetrically along the $\mathrm{x}$ direction. Correspondingly, the droplet will shrink symmetrically along the y direction. The brighter images on the left are the sketches of top view of the droplets with magnetic field strength at $0 \mathrm{mT}$ and $370 \mathrm{mT}$. This chapter emphasizes on the investigation of the elongation behaviour. In addition, the base diameter of the droplet is an important parameter in digital microfluidics. So our experimental testing and analysis focus on $\mathrm{x}-\mathrm{z}$ plane only. Figure 3-7 shows the measured geometric parameters (height and width) as a function of the flux densities for the droplet with different volumes. The size of the droplet does not affect the trend of the curve. The results show that a stronger magnetic field would stretch the ferrofluid droplet, increasing the width and decreasing the height. It is worth noting that the width and height of the droplet remains constant upon reaching the saturated magnetization ( $B \approx 150 m T$ ). The solid lines are fitting curves. Hysteresis phenomena were presented in the behaviour of the droplets. To investigate the hysteresis effect in the experiment, data were recorded for both magnetic loading and unloading processes. The arrows in Figure 3-7 indicate the loading process with increasing flux density and the unloading process with decreasing flux density. The intrinsic hysteresis in the system can be investigated by the discrepancy between the two curves. 

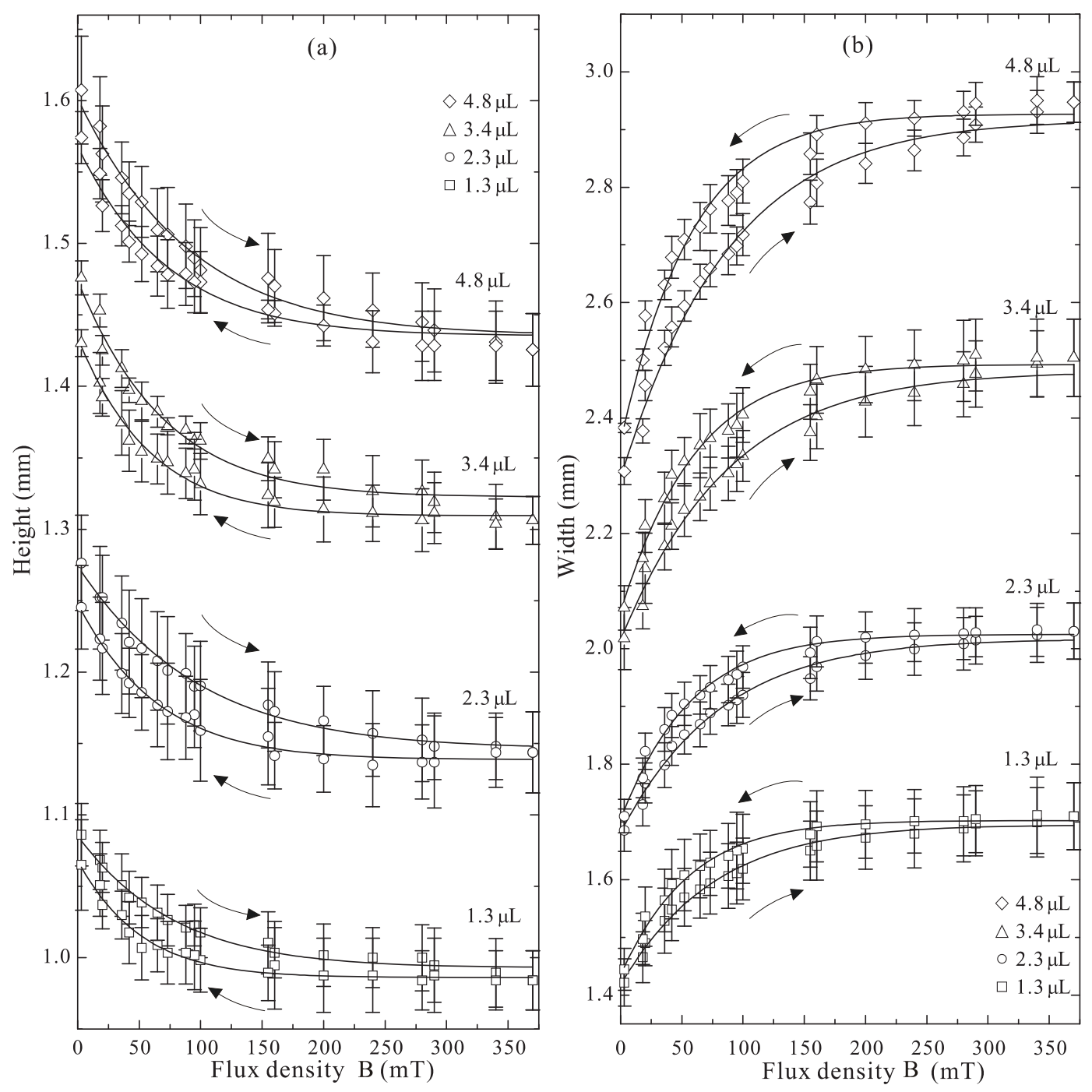

Figure 3-7: The volume of the droplets varies from $1.3 \mu \mathrm{L}$ to $4.8 \mu \mathrm{L}$. Experimental results of droplet parameters as a function of flux density B: (a) height; (b) width.

The magnetization of the ferrofluid can be obtained from Figure 3-5 for the corresponding magnetic flux density. Figure 3-8 shows the parameters of droplet as a function of magnetization. The specific mass magnetization values in Figure 3-8 were obtained through fourth-order Newton's interpolating polynomials according to the data of magnetization shown in Figure 3-5. 

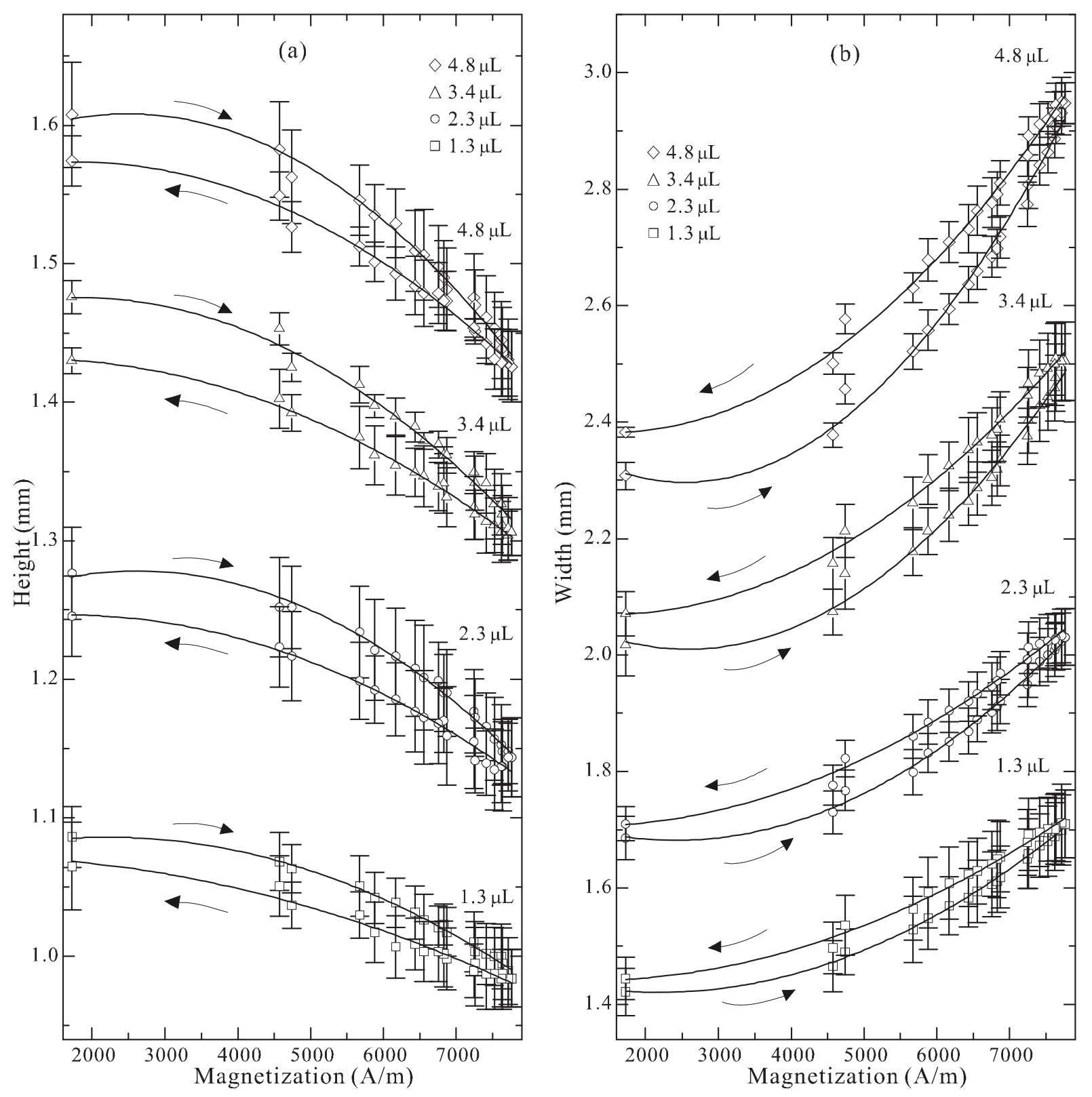

Figure 3-8: The volume of the droplets varies from $1.3 \mu \mathrm{L}$ to $4.8 \mu \mathrm{L}$. Experimental results of droplet parameters as a function of magnetization (with 2nd-order polynomial fit): (a) height; (b) width.

The Langevin's magnetization law as shown in Figure 3-5 can systematically describe the super paramagnetic properties in the ferrofluid. Because of the large magnetic flux density, the droplet in the experiment actually underwent nonlinear magnetization. For a small flux density, the deformation of droplet is significantly affected by the applied flux density. For the flux density less than $150 \mathrm{mT}$, Figure 3-5 
shows that the magnetization increases dramatically with an increasing flux density. In the range of flux density from $150 \mathrm{mT}$ to $370 \mathrm{mT}$, the characteristic parameters of the droplets remain almost constant if depicted as a function of the flux density. The relationship between the droplet parameters and the magnetization is better represented using the actual magnetization data of the ferrofluid droplet.

\subsection{Numerical Simulation}

The numerical simulation has been carried out to calculate the deformation of a ferrofluid droplet in a uniform magnetic field. The simulation results are compared with the experimental measurements.

\subsubsection{Governing Equations}

The two liquids are considered to be immiscible. For the two phase flow, oil is assumed to always wet the solid surface. The contact of ferrofluid with the solid wall is neglected in the numerical calculation. Except for the jump at the interface, the properties of a two-phase system are assumed to be continuous. Thus, the governing equations of a continuum are still valid for describing the physical field inside each phase. The continuity equation and the momentum equations are expressed as,

$$
\begin{gathered}
\frac{\partial \rho}{\partial t}+\nabla \cdot(\rho \vec{u})=0 \\
\frac{\partial}{\partial t}(\rho \vec{u})+\nabla \cdot(\rho \vec{u} \vec{u})=-\nabla P+\nabla \cdot\left[\mu\left(\nabla \vec{u}+\nabla \vec{u}^{T}\right)\right]+\vec{F}
\end{gathered}
$$

The continuum assumption is applied for liquid properties. The liquid is considered to be incompressible. The mass, energy and momentum are assumed to conserved for a 
fixed volume. By assuming an incompressible, unsteady, viscous, immiscible multiphase system, the governing equations become,

$$
\begin{gathered}
\rho \nabla \cdot(\vec{u})=0 \\
\rho \frac{\partial \vec{u}}{\partial t}+\rho \nabla \cdot(\vec{u} \vec{u})=-\nabla P+\mu \nabla \cdot\left(\nabla \vec{u}+\nabla \vec{u}^{T}\right)+\vec{F}
\end{gathered}
$$

where $\vec{u}$ is the velocity vector, and $P$ is the pressure. For the case of a ferrofluid droplet under the effect of magnetic force, the body force per unit volume $\vec{F}$ consists of gravitational force $\vec{F}_{g}$, interfacial tension force $\vec{F}_{\sigma}$ [189-190] and magnetic force $\vec{F}_{m}$ [191]. Besides, the magnetic field also needs to be calculated. The governing Maxwell's differential equations are Eq. (2.10), Eq. (2.13) and,

$$
\begin{gathered}
\nabla \times \vec{E}=-\frac{\partial \vec{B}}{\partial t} \\
\nabla \times \vec{H}=\vec{J}_{f}+\frac{\partial \vec{D}}{\partial t}
\end{gathered}
$$

where $\vec{J}_{f}$ is the free current density. The electromagnetic induction is not considered as the uniform magnetic field is relatively stable. The coupling of magnetic field and electric field in Eq. (3.9) and Eq. (3.10) is neglected. As a result, magnetic potential $\psi$ is incorporated into the model according to the following equation [191],

$$
\nabla \bullet\left[\left(1+\chi_{m}\right) \nabla \psi\right]=0
$$

where $\chi_{m}$ is the ferrofluid susceptibility, and $\psi$ is the magnetic scalar potential. The magnetic potential is introduced in a form of $\vec{H}=-\nabla \psi$. The boundary condition for the magnetic field is,

$$
\vec{H} \cdot \hat{n}=-\frac{\partial \psi}{\partial n}, \forall \vec{x} \in \partial \Omega
$$


where $\Omega$ is the whole computational domain, $n$ is the normal vector, and $\hat{n}$ is the unit normal vector. For a magnetizable liquid, the Langevin's magnetization function in Eq. (3.4) is assumed and incorporated into the Maxwell equations. The susceptibility in Eq. (3.11) is taken to be [188],

$$
\chi_{m}=\frac{M_{s}}{H}\left(\operatorname{coth}\left(\frac{3 \chi_{0}}{M_{s}} H\right)-\frac{1}{\left(\left(3 \chi_{0} / M_{s}\right) H\right)}\right)
$$

Due to discontinuity across the interface, properties within the whole computational domain are defined using the smoothed Heaviside function $\Theta(\phi)$ through arithmetic mean,

$$
\alpha(\phi)=\Theta \alpha_{+}+(1-\Theta) \alpha_{-}
$$

or a harmonic mean,

$$
\frac{1}{\alpha(\phi)}=\frac{\Theta}{\alpha_{+}}+\frac{(1-\Theta)}{\alpha_{-}}
$$

The smoothed Heaviside function $\Theta(\phi)$ is defined as [190],

$$
\Theta(\phi)= \begin{cases}0 & \phi<-\varepsilon, \\ (\phi+\varepsilon) /(2 \varepsilon)+\sin (\pi \phi / \varepsilon) /(2 \pi) & -\varepsilon \leq \phi \leq \varepsilon, \\ 1 & \varepsilon<\phi,\end{cases}
$$

The typical good value of $\varepsilon$ is 1.5 of the grid cell length [192]. In our numerical model, the density of liquid was calculated by the arithmetic method. The viscosity and magnetic susceptibility act as coefficient of diffusion term and can be solved through the harmonic approach. 


\subsubsection{Numerical Methods}

The governing equations of the fluid flow and the magnetic field follow the general transport equation,

$$
\underbrace{\partial(\rho \Phi) / \partial t}_{\text {unsteady term }}+\underbrace{\nabla \bullet(\rho \vec{u} \Phi)}_{\text {convection term }}=\underbrace{\nabla \bullet(\Gamma \nabla \Phi)}_{\text {diffusion term }}+\begin{gathered}
S \\
\text { source term }
\end{gathered}
$$

where $\Phi$ is the dependent variable which can be velocity, magnetic potential, temperature and a displacement component of a linear-elastic isotropic material. $\Gamma$ is the diffusion coefficient. $S$ stands for the source term. The equations in the form of Eq. (3.17) can be solved numerically. The governing equations are solved on a Cartesian staggered grid by FVM. The FVM is a method for solving partial difference equations. Traditionally, the solution of the partial equation consists of a set of numbers from which the dependant variable can be determined. However, the procedure is not convenient for cases mainly interested in the values at various locations. Following other numerical method, FVM employs the dependent values at several given points as the primary unknowns. As shown in Figure 3-9, the computational domain is divided into small volume surrounding each grid point on a mesh. The solid lines are the grid lines. Each grid point is the center of a control volume. The scale variables are stored at the grid points, while the velocities are stored at the control volume surfaces. FVM includes the formulating algebraic equations for the unknowns and developing algorithms for solving them. Discretization (spatial and temporal) methods are used to get the discrete dependent values at the grid points. Wisely selection of iterative method needs to be made for solving the algebraic 
equations. The number of repetitions should be determined since the solution procedure is iterative. To control the number of repetitions, the maximum number of repetitions and the local relative error are defined. The local relative error is defined as the ratio between the residual of the discretization equation and the largest of all the terms in the discretization equation. When the local relative error is less than or equal to a very small number $\left(1 \times 10^{-8}\right.$ in our code $)$, the iteration will stop.

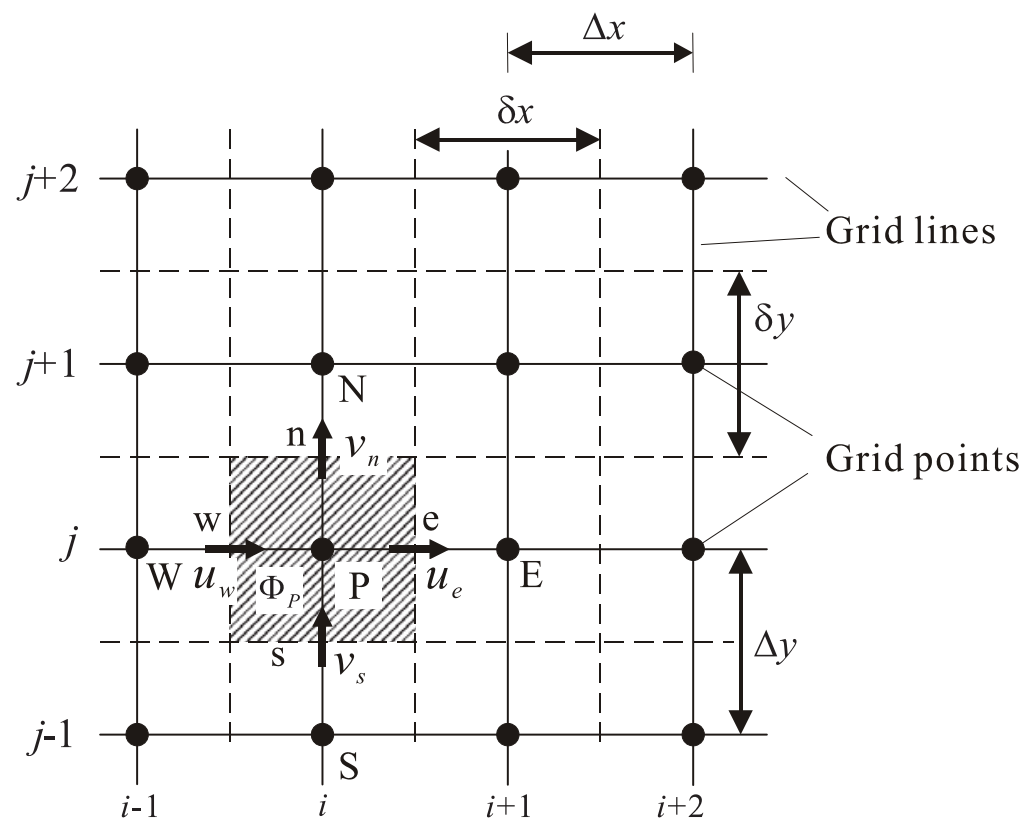

Figure 3-9 Grids and control volumes for two dimensional discretization.

The level-set method [193] is used to track the interface $\Gamma_{i}$ between the ferrofluid and the surrounding oil. The motion of the interface is obtained through introducing a signed normal distance function $\phi(\vec{x}, t)$ from the interface,

$$
\phi(\vec{x}, t)=\left\{\begin{array}{c}
<0, \text { if } \vec{x} \in \Omega_{-} \\
0, \text { if } \vec{x} \in \Gamma_{i} \\
>0, \text { if } \vec{x} \in \Omega_{+}
\end{array}\right.
$$


where the subscripts (+) and (-) represent two immiscible phases. As shown in Figure $3-10$, the interface $\Gamma_{i}$ is defined by the zero level-set of function $\phi(\vec{x}, t)$. The values of variable are positive inside the interface, and negative outside the interface. As a result, the two different fluids and the interface can be distinguished.

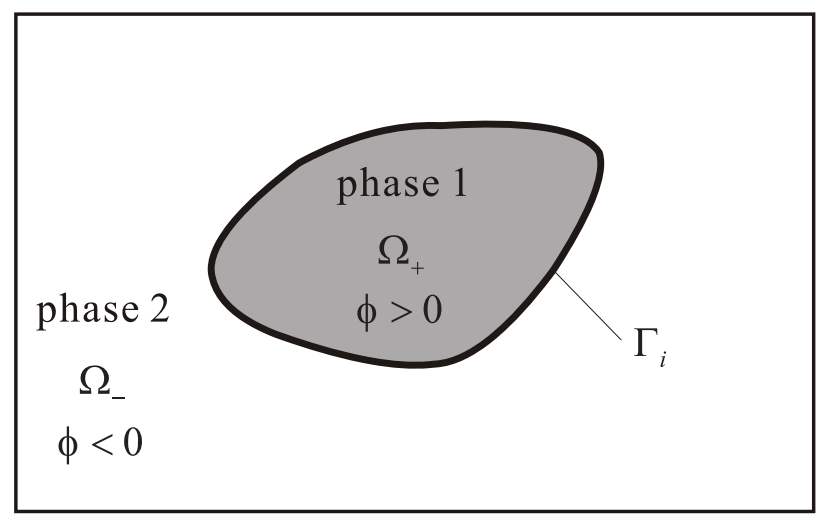

Figure 3-10 The whole computational domain.

The motion of the interface can be obtained by taking the time derivative of $\phi(\vec{x}, t)=0$,

$$
\frac{\partial \phi}{\partial t}+\vec{u} \bullet \nabla \phi=0
$$

This level-set function can be solved within a narrow band around the interface. This narrow-band approach (Peng et al. [194]) showed that the accuracy of the numerical results was not affected. The processing time would be reduced by one order of magnitude due to the application of the narrow-band approach. The signed normal distance function $\phi(\vec{x}, t)$ always satisfies $|\nabla \phi|=1$. During the numerical calculation, a reinitialization strategy is applied to reset and ensure the level-set function to be a signed distance function of the interface [195]. In this code, the reinitialization equation for the correction of $\phi$ at time $t^{*}$ is given as [196], 


$$
\begin{gathered}
\frac{\partial \phi^{*}}{\partial t^{*}}=\operatorname{sign}\left(\phi_{0}\right)\left(1-\left|\nabla \phi^{*}\right|\right) \\
\phi_{0}(\vec{x})=\phi(\vec{x}, 0) \\
\operatorname{sign}\left(\phi_{0}\right)=\phi / \sqrt{\phi^{2}+|\nabla \phi|^{2} \Delta x}
\end{gathered}
$$

The level-set equation Eq. (3.19) and reinitialization equation Eq. (3.20) are special form of the Hamiltonian-Jacobi equation,

$$
\phi_{t}+H_{1}(\nabla \phi)=0
$$

where $H_{1}(\nabla \phi)$ is the Hamiltonian. Eq. (3.23) becomes the level-set equation Eq. (3.19) when $H_{1}(\nabla \phi)$ takes the form of convection in an externally generated velocity field,

$$
H_{1}(\nabla \phi)=\vec{u} \bullet \nabla \phi
$$

The numerical approximation of Hamiltonian in three spatial dimensions $H\left(\phi_{x}, \phi_{y}, \phi_{z}\right)$ is implemented by the local Lax-Friedrichs schemes (LLF) scheme [197]. The numerical Hamiltonian $\hat{H}^{L L F}\left(\phi_{x}^{-}, \phi_{x}^{+}, \phi_{y}^{-}, \phi_{y}^{+}, \phi_{z}^{-}, \phi_{z}^{+}\right)$has the form of,

$$
\begin{aligned}
\hat{H}^{L L F}=H\left(\frac{\phi_{x}^{+}+\phi_{x}^{-}}{2}, \frac{\phi_{y}^{+}+\phi_{y}^{-}}{2}, \frac{\phi_{z}^{+}+\phi_{z}^{-}}{2}\right) \\
-\alpha^{x}\left(\frac{\phi_{x}^{+}-\phi_{x}^{-}}{2}\right)-\alpha^{y}\left(\frac{\phi_{y}^{+}-\phi_{y}^{-}}{2}\right)-\alpha^{z}\left(\frac{\phi_{z}^{+}-\phi_{z}^{-}}{2}\right) \\
\alpha^{x}=\max \left|H_{\phi_{x}}\left(\phi_{x}, \phi_{y}, \phi_{z}\right)\right| \\
\alpha^{y}=\max \left|H_{\phi_{y}}\left(\phi_{x}, \phi_{y}, \phi_{z}\right)\right| \\
\alpha^{z}=\max \left|H_{\phi_{z}}\left(\phi_{x}, \phi_{y}, \phi_{z}\right)\right|
\end{aligned}
$$

Here, $\alpha^{x}, \alpha^{y}$ and $\alpha^{z}$ are set to the maximum values of $|u|,|v|$, and $|w|$ in the neighboring grid points since $H_{\phi_{x}}=u, H_{\phi_{y}}=v$ and $H_{\phi_{z}}=w$. 
Eq. (3.23) becomes the reinitialization equation Eq. (3.20) when $H_{1}(\nabla \phi)$ takes the form of,

$$
H_{1}(\nabla \phi)=\operatorname{sign}\left(\phi_{0}\right)(|\nabla \phi|-1)
$$

The numerical approximation of Hamiltonian in three spatial dimensions $H\left(\phi_{x}, \phi_{y}, \phi_{z}\right)$ is implemented by the Godunov's scheme [197]. The numerical Hamiltonian $\hat{H}^{G}\left(\phi_{x}^{-}, \phi_{x}^{+}, \phi_{y}^{-}, \phi_{y}^{+}, \phi_{z}^{-}, \phi_{z}^{+}\right)$has the form of, $\hat{H}^{G}=\left\{\begin{array}{l}s\left\{\sqrt{\left[\max \left(\left\langle\phi_{x}^{+}\right\rangle^{-},\left\langle\phi_{x}^{-}\right\rangle^{+}\right)\right]^{2}+\left[\max \left(\left\langle\phi_{y}^{+}\right\rangle^{-},\left\langle\phi_{y}^{-}\right\rangle^{+}\right)\right]^{2}+\left[\max \left(\left\langle\phi_{z}^{+}\right\rangle^{-},\left\langle\phi_{z}^{-}\right\rangle^{+}\right)\right]^{2}}-1\right\}, \text { if } \phi_{i j k}^{o} \geq 0 \\ s\left\{\sqrt{\left[\max \left(\left\langle\phi_{x}^{+}\right\rangle^{+},\left\langle\phi_{x}^{-}\right\rangle^{-}\right)\right]^{2}+\left[\max \left(\left\langle\phi_{y}^{+}\right\rangle^{+},\left\langle\phi_{y}^{-}\right\rangle^{-}\right]^{2}+\left[\max \left(\left\langle\phi_{z}^{+}\right\rangle^{+},\left\langle\phi_{z}^{-}\right\rangle^{-}\right]^{2}\right.\right.}-1\right\}, \text { otherwise }\end{array}\right.$

$$
\begin{aligned}
& \langle a\rangle^{+}=\max (a, 0) \\
& \langle a\rangle^{-}=-\min (a, 0) \\
& s=\operatorname{sign}(\phi)
\end{aligned}
$$

where the spatial derivatives $\phi_{x}^{-}, \phi_{x}^{+}, \phi_{y}^{-}, \phi_{y}^{+}, \phi_{z}^{-}$and $\phi_{z}^{+}$are discretized with a highorder weighted essentially non-oscillatory (WENO) scheme [197-198]. The total variation diminishing (TVD) Runge-Kutta method was used for the time discretization [199-200].

In order to alleviate the volume loss problem by the level-set method, marker particles are employed close to the interface. The spherical marker particles present the characteristic information which can be used to rebuild the level set function. This is the particle level-set method (PLS) [201-202]. The radius of the particles ranges from $r_{\min }=0.1 \Delta x$ to $r_{\max }=0.5 \Delta x . \Delta x$ is the grid size which is shown in Figure 3-9. Two sets of particles are seeded as there is characteristic information on both sides of the 
interface. Both types of particles are randomly seeded within a band between a distance of $b_{\min }=r_{\min }$ and $b_{\max }$ of the interface. The maximum band width $b_{\max }$ takes the value of $2 \Delta x$ in $3 \mathrm{D}$ computation and $3 \Delta x$ in $2 \mathrm{D}$ computation. The initial randomly distributed particles and the interface in a $2 \mathrm{D}$ computation are presented in Figure 3-11(a). The initial location of the particle is $\vec{x}_{p}$ on the $\phi=\phi\left(\vec{x}_{p}\right)$ isocontour. The marker particles are attracted in the normal direction to the desired isocontour $\phi_{g}$ at the appropriate side of the interface by using the equation,

$$
\vec{x}_{n e w}=\vec{x}_{p}+\left[\phi_{g}-\phi\left(\vec{x}_{p}\right)\right] \vec{N}\left(\vec{x}_{p}\right)
$$

where $\vec{x}_{n e w}$ and $\vec{N}\left(\vec{x}_{p}\right)$ are the new position of the particle and the unit normal vector.

Figure 3-11(b) shows the successful particle attraction.

(a)

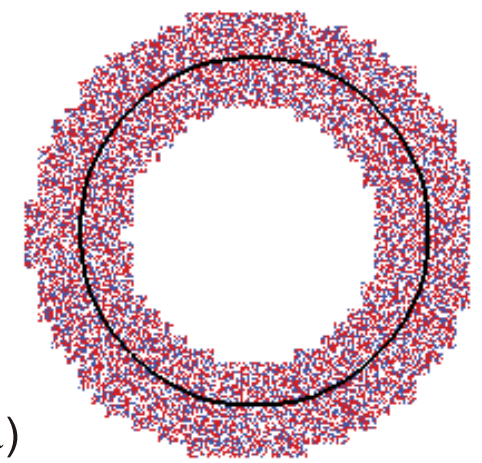

(b)

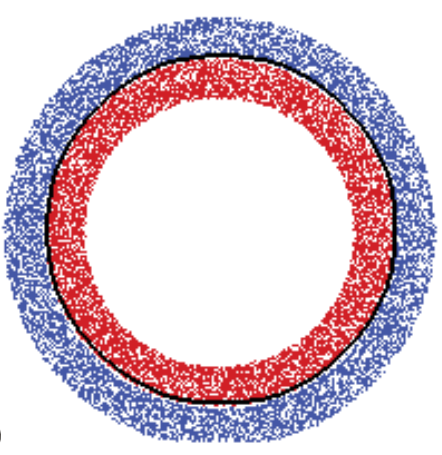

Figure 3-11 The interface and the seeded particles (positive particles in blue and negative particles in red): (a) Random seeding; (b) Particles after successful attraction.

The advection of particles is governed by,

$$
\frac{d \vec{x}_{p}}{d t}=\vec{u}\left(\vec{x}_{p}\right)
$$

where $\vec{u}\left(\vec{x}_{p}\right)$ is the velocity of particle. The velocities of particles are obtained by using trilinear interpolation of fluid velocities. The second-order TVD Runge-Kutta scheme is employed to evolve the particle positions change with time. In smooth well- 
resolved regions, the particles do not drift significantly across the interface as the level-set solution is highly accurate. However, escaped particles would appear because of numerical errors. A particle is considered as escaped when it is on the wrong side of the interface by more than its radius. Particle correction strategies are required to rebuild the $\phi>0$ region using escaped positive particles, and $\phi \leq 0$ region using escaped negative particles. Sets of escaped positive and negative particles are defined as $E_{+}$and $E_{-}$. The level set functions associated with opposite singed particles $\phi_{-}$ and $\phi_{+}$, are initialized with $\phi$ and then calculated as,

$$
\begin{gathered}
\phi_{+}=\left\{\begin{array}{l}
\max _{\forall p \in E_{+}}\left(\left|\phi_{+}\right|,\left|\phi_{p}\right|\right), \text { if } \phi_{+}>0 \\
-\min _{\forall p \in E_{+}}\left(\left|\phi_{+}\right|,\left|\phi_{p}\right|\right), \text { if } \phi_{+}<0
\end{array}\right. \\
\phi_{-}=\left\{\begin{array}{l}
\min _{\forall p \in E_{-}}\left(\left|\phi_{-}\right|,\left|\phi_{p}\right|\right), \text { if } \phi_{-}>0 \\
-\max _{\forall p \in E_{-}}\left(\left|\phi_{-}\right|,\left|\phi_{p}\right|\right), \text { if } \phi_{-}<0
\end{array}\right.
\end{gathered}
$$

where $\phi_{p}=\operatorname{sign}\left[\phi\left(\vec{x}_{p}\right)\right]\left(r_{p}-\left|\vec{x}-\vec{x}_{p}\right|\right) \cdot \phi\left(\vec{x}_{p}\right)$ is the trilinear interpolated level-set value from the values underlying the grid points. Then, the level-set function $\phi$ is set as,

$$
\phi= \begin{cases}\phi_{-}, & \text {if }\left|\phi_{+}\right|>\left|\phi_{-}\right| \\ \phi_{+}, & \text {if }\left|\phi_{+}\right| \leq\left|\phi_{-}\right|\end{cases}
$$

Particle reseeding is necessary as some regions are short of particles due to interface shift and particle attraction. The particles are deleted if they are in a cell outside of the narrow band $\left(b_{\max }\right)$. Or if the number of particles is less than 64 in a 3D case, particles are added to the cell. If there are more than 64 non-escaped particles in a cell within the narrow band, the value of sign $\left[\phi\left(\vec{x}_{\text {new }}\right)\right] \phi\left(\vec{x}_{\text {new }}\right)-r_{p}$ are calculated 
and stored in a heap data structure. This heap data structure can holds 64 values in descending order. For remaining particles, a heap sort is performed to delete particles with larger value of $\operatorname{sign}\left[\phi\left(\vec{x}_{\text {new }}\right)\right] \phi\left(\vec{x}_{\text {new }}\right)-r_{p}$.

\subsubsection{Validations and Results}

\section{Grid-independent study}
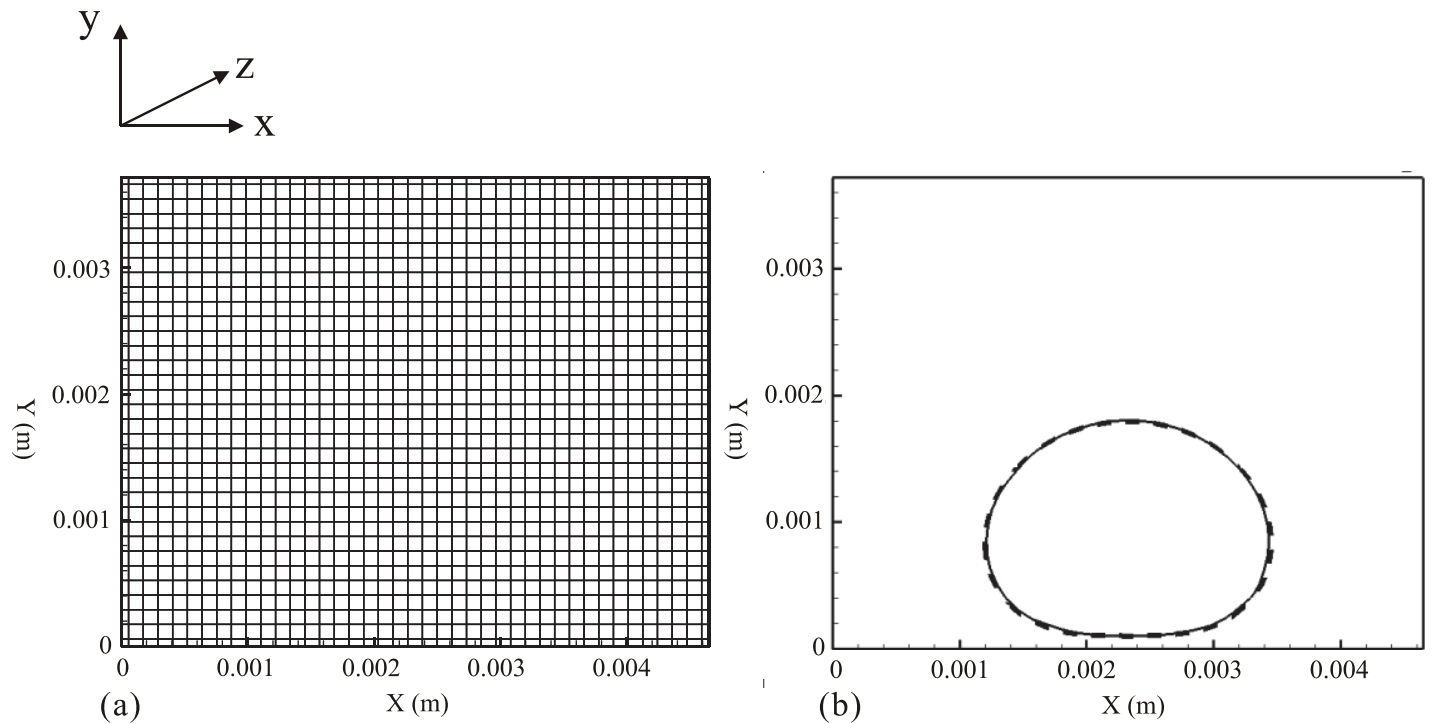

Figure 3-12 The computational domain: (a) Uniform meshes; (b) Grid-independent study with droplet in the computational domain (solid line: $42 \times 34 \times 26 \mathrm{CVs}$ at a time step of $\Delta t=1 \times 10^{-3} \mathrm{~s}$, dashed line: $62 \times 50 \times 38 \mathrm{CVs}$ at a time step of $\Delta t=5 \times 10^{-4} \mathrm{~s}$ ).

A 3D numerical model was developed using the geometry from the experiment for a ferrofluid droplet surrounded by mineral oil. The initial size of the simulated droplet was set to be $4.8 \mu \mathrm{L}$, which was the largest size investigated in the experiment. For this size, the effect of gravity was also captured in the simulation. The whole computational area was meshed uniformly Figure 3-12(a). Due to symmetry, a onehalf model was implemented. No-slip boundary condition was employed at the wall. Symmetric boundary conditions were applied at the symmetric surface. At first, a grid- 
independent study was carried out. Two different meshes with $42 \times 34 \times 26 \mathrm{CV}$ s at a time step of $\Delta t=1 \times 10^{-3} \mathrm{~s}$ and $62 \times 50 \times 38 \mathrm{CVs}$ at a time step of $\Delta t=5 \times 10^{-4} \mathrm{~s}$ were used. Figure 3-12(b) shows that the difference between the solutions is relatively small. Thus, the mesh $42 \times 34 \times 26 \mathrm{CVs}$ at a time step of $\Delta t=1 \times 10^{-3} \mathrm{~s}$ was sufficient precise to capture the droplet behaviour in the computational domain.

\section{Simulation without magnetic field}

At first, the interfacial tension between the ferrofluid and the surrounding mineral oil was obtained by matching the shapes of the droplet from experiment and simulation without the magnetic effect. Without the magnetic force, the deformation of droplet was determined by the gravitational force and interfacial tension force only. The simulation coupled the interactions between gravitational forces and interfacial forces in the model. The numerical calculations were implemented for different interfacial tension forces with other system parameters fixed. A constant value of interfacial tension between the ferrofluid and the surrounding medium was assumed. The value of the interfacial tension was determined when the numerically obtained droplet shape matches the experimental shape. By using this fitting method, the interfacial tension between the ferrofluid and the mineral oil was determined as 3.0 $\times 10^{-3} \mathrm{~N} / \mathrm{m}$ in this case.

Figure 3-13 shows the comparison between experimental and simulation results of the droplet shape in the absence of a magnetic field. A thin oil film prevents the droplet from wetting the bottom surface. The numerical result is shown in Figure 
3-13(b) where the solid black line represents the interfacial curve. The developed numerical model can accurately predict the deformation of the droplet considering the effects of gravity and interfacial forces.

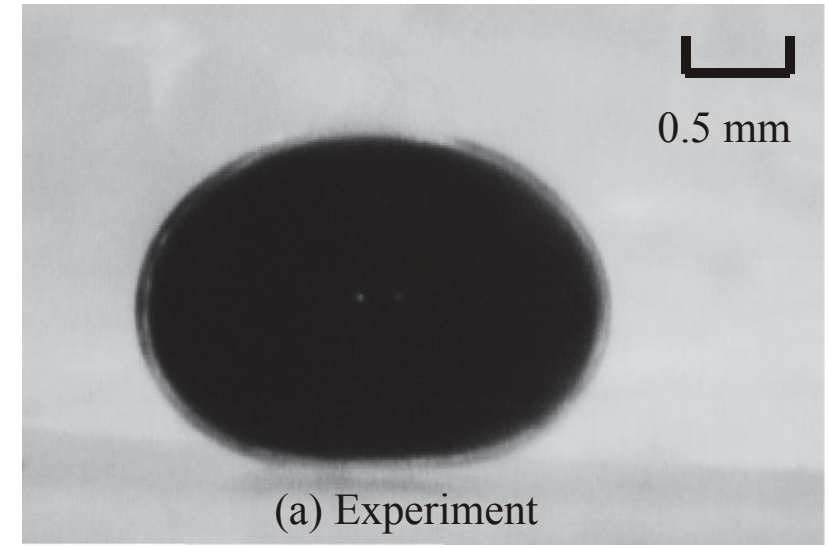

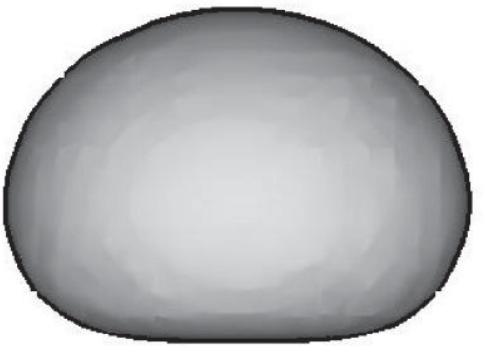

(b) Simulation

Figure 3-13: Comparison between experimental and numerical results (gravity flattened effect without magnetic force): (a) experiment; (b) simulation.

\section{Simulation with magnetic field}

The magnetic force was then induced to study the effect on droplet deformation. The susceptibility mismatch between the two fluids enabled the generation of magnetic force and thus the droplet deformation. In the experiments, the deformation of ferrofluid droplets occurred under a relatively high field strength. The magnetization curve in Figure 3-5 shows that the region $(B=0 \sim 150 \mathrm{mT}$ ) for droplets deformation is highly nonlinear. As ferrofluid droplets underwent nonlinear magnetization, the Langevin's magnetization law described by Eq. (3.4) was assumed and incorporated into the governing equation of the numerical model. Through numerical solution of Eq. (3.11), the magnetic potential was obtained shown in Figure 3-14. The arrow in Figure 3-14 indicates the direction of the magnetic field $H_{0}$. The 
initial shape and the stretched shapes of the ferrofluid droplet are sketched in Figure 3-14.

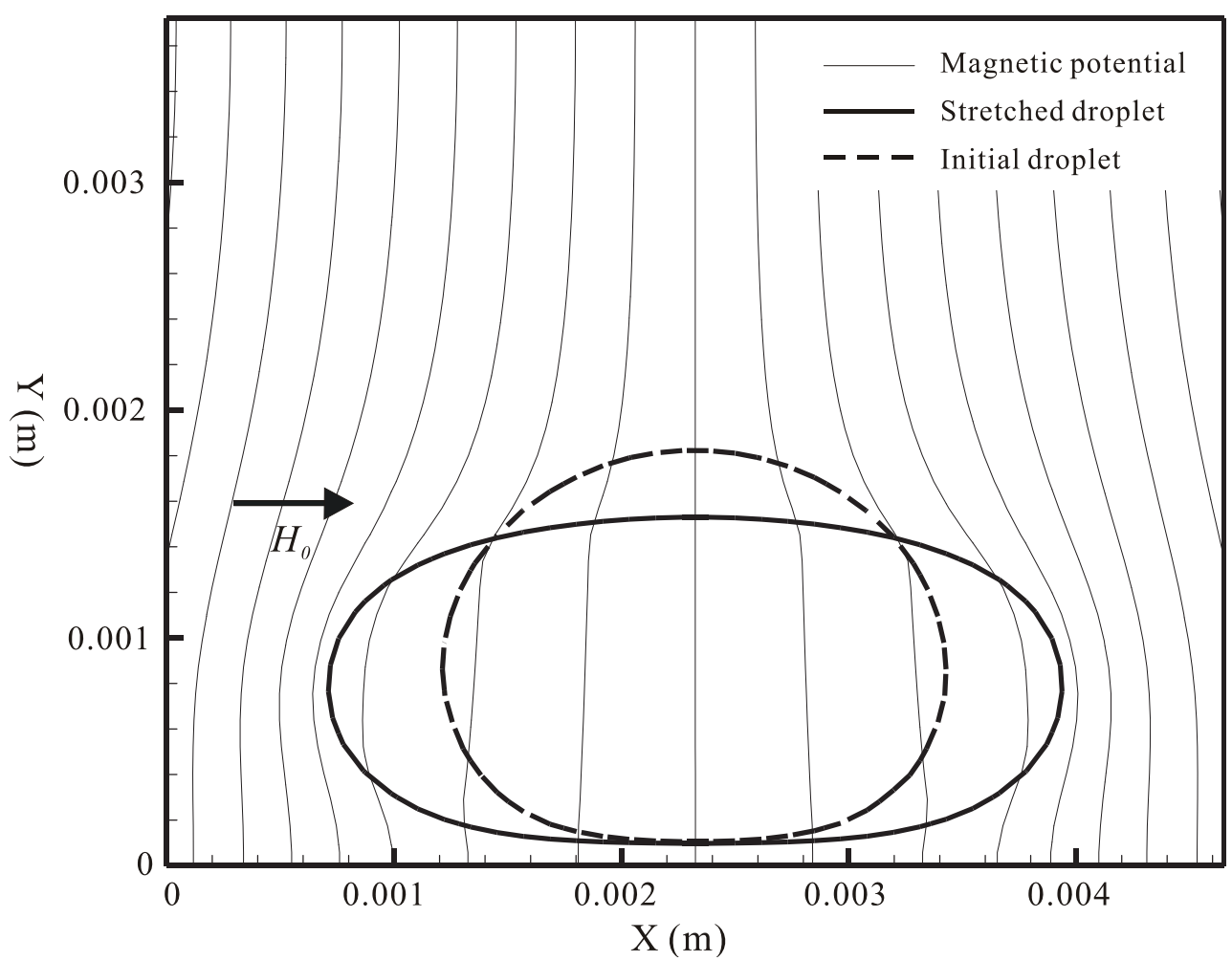

Figure 3-14: The magnetic potential for a sessile ferrofluid droplet in a uniform magnetic field.

The deformation of the droplets depends on both the magnetic susceptibility and the magnetic field strength. To generalize the investigation, the calculation was implemented with the magnetic Bond numbers $B_{m}$, which can be calculated from the properties of droplets and the applied magnetic field. The magnetic Bond number is defined as the ratio of magnetic force to interfacial tension force,

$$
B_{m}=\frac{\mu_{0} \chi_{m} V^{1 / 3} H_{0}^{2}}{2 \sigma}
$$

where $H_{0}$ is the applied magnetic field intensity, $\chi_{m}$ is the ferrofluid susceptibility, and $\sigma$ is the interfacial tension between the ferrofluid and the mineral oil. The volume 
of the droplet $V$ is controllable in both experimental and numerical studies. The magnitude of the magnetic Bond number varies as the magnetic field strength changes.

Figure 3-15 compares the experimental results with the numerical results for a $4.8-\mu \mathrm{L}$ ferrofluid droplet. The deformations of the $4.8-\mu \mathrm{L}$ droplet observed in the experiments are shown in Figure 3-15(a).

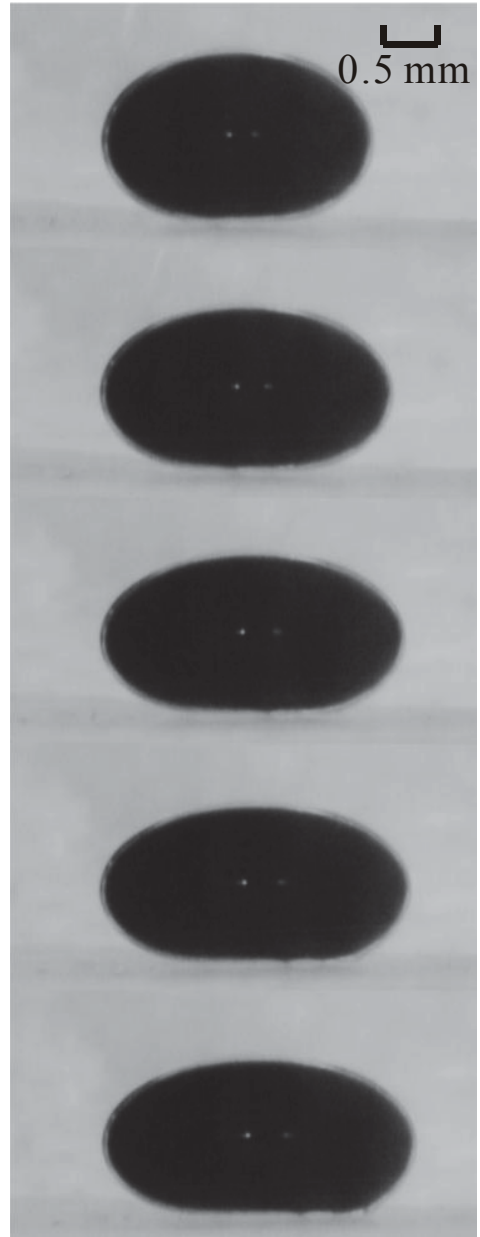

(a) Experiment
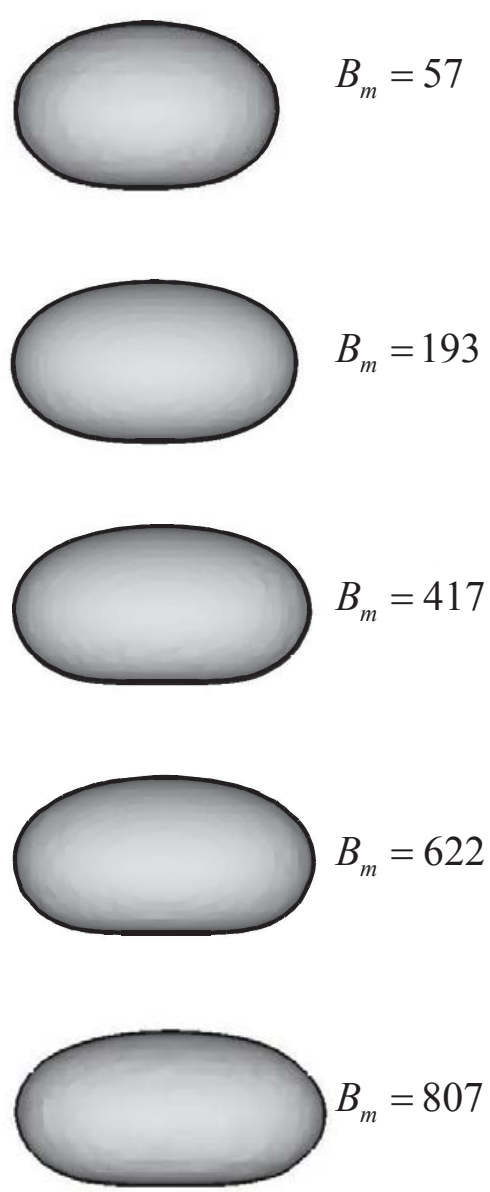

(b) Simulation

Figure 3-15: The deformation of a ferrofluid droplet under different magnetic Bond numbers $(V=4.8 \mu \mathrm{L})$ : (a) experiment; (b) simulation. 

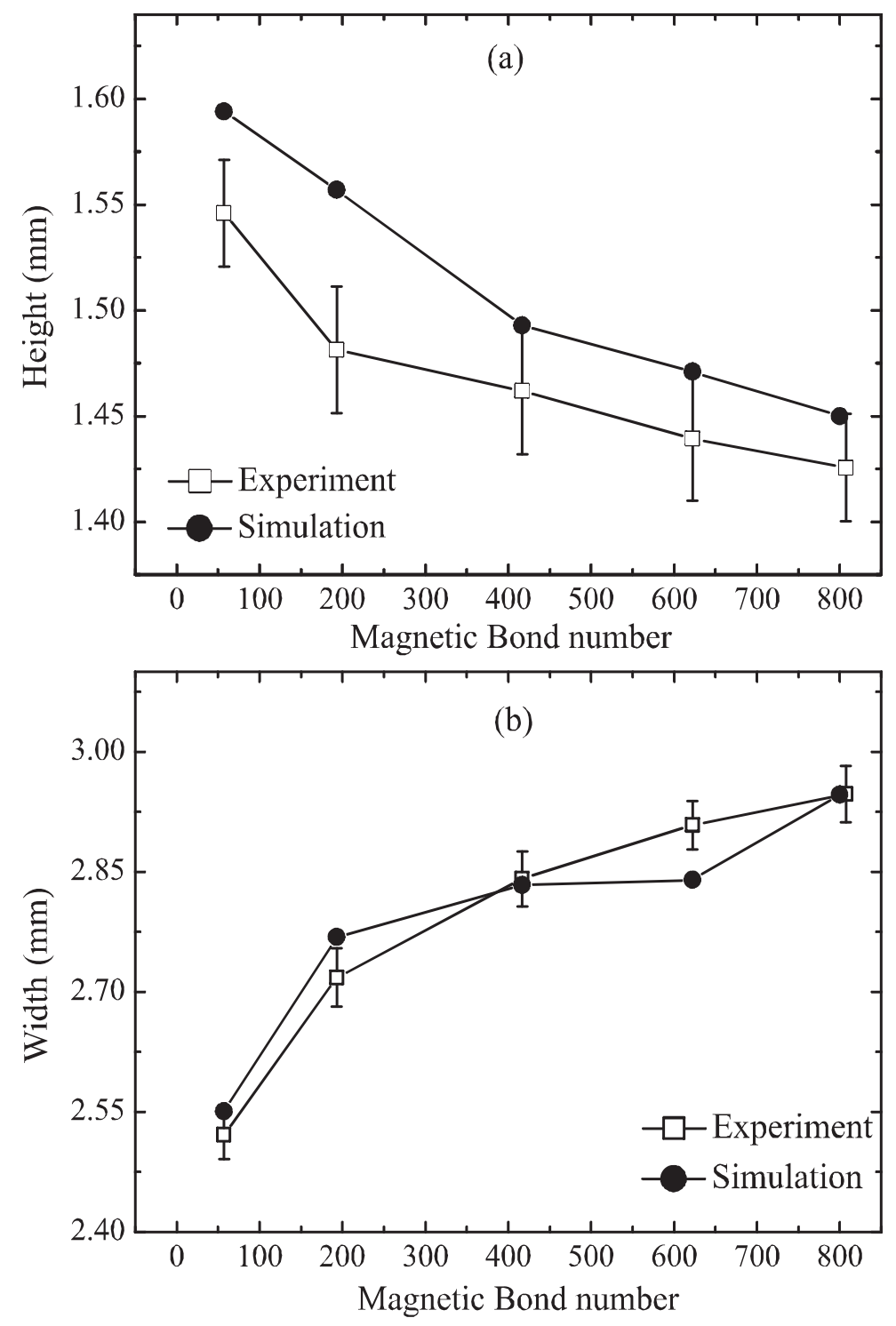

Figure 3-16: Geometrical parameters as functions of magnetic Bond number of a $4.8-\mu \mathrm{L}$ ferrofluid droplet: (a) height; (b) width.

The numerical calculation was implemented with the value of magnetic Bond number corresponding to those used in the experiments, and the results are shown in Figure 3-15(b). It is seen that the shapes of the droplet from the experiments agree well with the interfacial curve obtained from the simulation for different Bond numbers. The change of the width and the height of the droplet are compared in Figure 3-16. The higher magnetic Bond number leads to a further stretching (width increase) 
of the droplet. The experimental results indicate that the stretching behaviour decreases as magnetic Bond number increases. This nonlinear behaviour agrees well with the trend predicted by the numerical calculation. Although the experimental and numerical results show the same trend, the values differ slightly. The droplet height of the simulation is slightly higher than that of the experiments. The discrepancy may be caused by the simplified numerical model.

\subsection{Summary}

This chapter presents the deformation of a sessile ferrofluid droplet on a super hydrophobic surface under the influence of an external uniform magnetic field. The effect of an applied magnetic field on the droplet geometry was investigated through both experiment and numerical simulation. The droplet geometry for different magnetic flux densities was investigated experimentally. For nonlinear magnetized ferrofluid droplets, the Langevin's magnetization law was used in the numerical model. The nonlinear characteristics of the deformation of the droplet were captured well in the numerical model. Both experiment and simulation confirm the nonlinear relationship between geometric parameters of the droplet and the magnetic Bond number. The good agreement verifies that the numerical model can be used for the study of magnetic droplets in general and ferrofluid droplets in particular. The results indicate that using an external uniform magnetic field is an effective method for studying behaviour of magnetic droplets. Furthermore, the results can be used for 
studying the phenomenon of magnetowetting or magnetic control of wettability for lab-on-a-chip applications [203]. 


\section{CHAPTER 4}

\section{MAGNETOWETTING AND SLIDING MOTION OF A SESSILE FERROFLUID DROPLET}

This chapter presents the investigation on the magnetowetting phenomena and the sliding motion of a ferrofluid droplet on a planar surface. Both experimental study and scaling analysis are conducted. The water-based ferrofluid in the study is a colloidal suspension of single-domain magnetic nanoparticles. Permanent magnets of different sizes and strengths are used to drag the droplet in a linear motion. The permanent magnet is attached to a translation stage to realize this motion. The investigation is initially focused on the magnetowetting effect. The apparent contact angle of a stationary sessile ferrofluid droplet is measured under different magnetic field strengths. Next, the dynamic contact angles and the sliding motion of the droplet are investigated. The ferrofluid droplet is first positioned and maintained in the stationary condition. The permanent magnet then approaches the ferrofluid droplet, traps it, and drags it. This experiment allows the observation of the hysteresis of the dynamic contact angles of the droplet. The evolution of the droplet shape and the change of the contact angles are captured and subsequently evaluated. Two operating regimes are expected in the experiment: steady droplet transport and disengagement of the magnet. The advancing and receding contact angles of the moving ferrofluid are measured at different moving speeds and magnetic field strengths. The measured contact angles are 
used to estimate the magnitude of the forces involved in the sliding motion. Scaling analysis is carried out to derive the critical velocity for droplet motion.

\subsection{Experimental Setup}

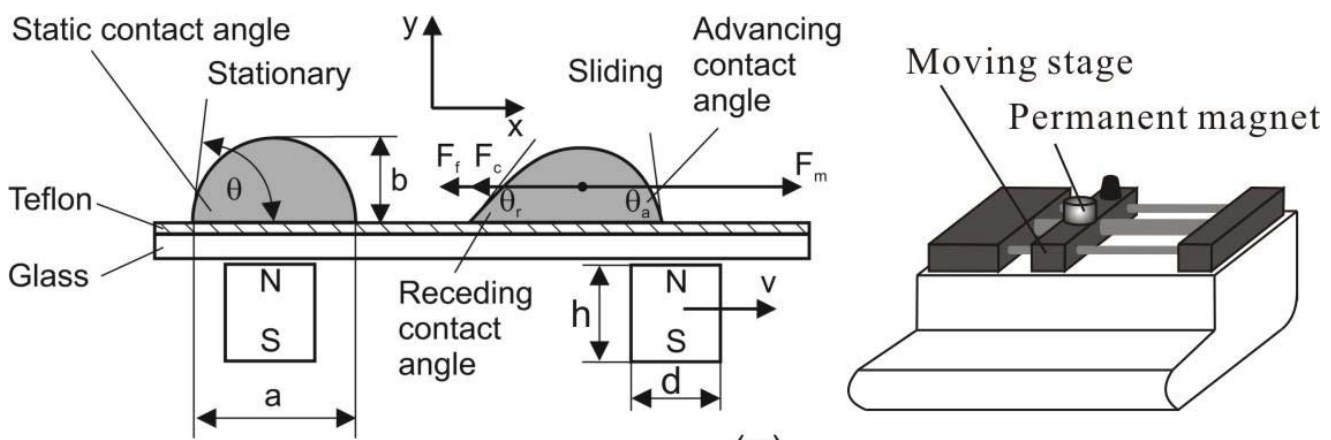

(a)

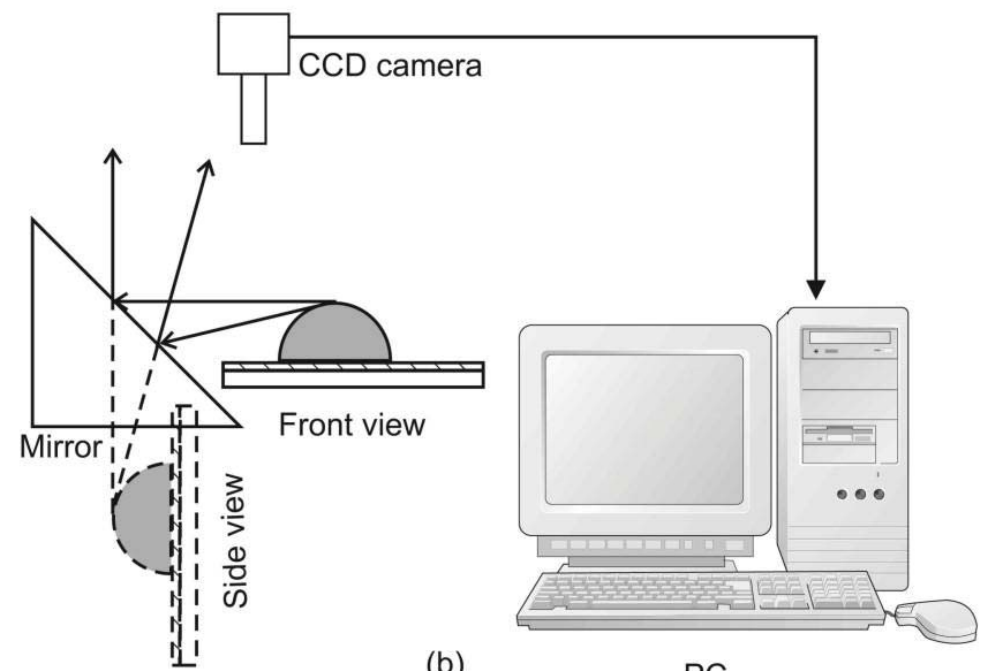

(b)

$$
\text { PC }
$$

Figure 4-1 Experimental setup: (a) Stationary and sliding ferrofluid droplet; (b) Imaging system for both front view and side view of the droplet (not to scale).

The experimental setup is illustrated in Figure 4-1. A ferrofluid droplet was dispensed on a solid surface which was hydrophobic because of a Teflon coating. The droplet would remain stationary if there was no driving force. By positioning a permanent magnet underneath the ferrofluid droplet, the magnetic driving force was induced to modify the wetting properties of the droplet. In order to program the motion, the permanent magnet was mounted on the moving stage of a syringe pump (KD 
Scientific, USA) as shown in Figure 4-1(a). The linear motion of the syringe pump was programmable; thus, the velocity of the permanent magnet could be controlled. With the help of a precision micrometer screw gauge, the top face of the permanent magnet was positioned accurately at a distance of $2 \mathrm{~mm}$ away from the hydrophobic surface. The Pyrex glass substrate was $0.5 \mathrm{~mm}$ thick. A gap of $1.5 \mathrm{~mm}$ between the lower surface of the glass slide and the top surface of the magnet was maintained. For calibration purpose, a plastic ruler was inserted into this gap.

The images of the ferrofluid droplet were captured with a CCD camera. The lens of the camera was positioned vertically to get the top view of the droplet. In order to obtain the top view and side view simultaneously, a $45^{\circ}$ mirror was placed close to the droplet. The imaging system was illustrated in Figure 4-1(b).

\subsection{Materials and Methods}

The hydrophobic surface was prepared follow the same procedure which has been introduced in chapter 3. Water-based ferrofluid (EMG508, Ferrotech, USA) with 1.8 vol $\% \mathrm{Fe}_{3} \mathrm{O}_{4}$ nanoparticles of $10 \mathrm{~nm}$ diameter was used in the experiments. The magnetization of the ferrofluid is saturated at a flux density of $6 \mathrm{mT}$. Its dynamic viscosity at $27^{\circ} \mathrm{C}$ is $\eta_{F F}=5 \mathrm{mPa} \cdot \mathrm{s}$. The density at $25^{\circ} \mathrm{C}$ is $\rho_{F F}=1.07 \times 10^{3} \mathrm{~kg} / \mathrm{m}^{3}$. The initial susceptibility is $\chi_{0}=0.24$. The surface tension $\sigma=31.66 \pm 0.05 \mathrm{mN} / \mathrm{m}$ was measured with a commercial tensiometer (TVT-2, measurement range of 0.1 to 100 $\mathrm{mN} / \mathrm{m}$, error of \pm 0.01-0.05 $\mathrm{mN} / \mathrm{m}$, Lauda, Germany). With these properties, the capillary length of the ferrofluid was calculated as $\ell=(\sigma /(\rho g))^{1 / 2}=1.74 \mathrm{~mm}$ [204]. 
For a critical Bond number $B o_{c r}=\rho g r_{c r}^{2} / \sigma=1$ [204], the critical radius of the droplet would be in the order of the capillary length $r_{c r}=\ell$. The base diameter of the droplets in the experiments was chosen as $a<2 \ell=3.48 \mathrm{~mm}$, so that the Bond number was below unity $(B o<1)$, and in the absence of the permanent magnet, the droplet could assume a spherical shape as shown in Figure 4-1(a). If $B o \gg 1$, the droplet was flattened to the shape which had a flat upper surface.

Three nickel-plate neodymium disc permanent magnets of different dimensions and strengths were purchased from Eclipse Magnetics (United Kingdom). The magnets were denoted here as small (N700-RB, $h=4 \mathrm{~mm}, d=2 \mathrm{~mm})$, medium (N701-RB, $h=2 \mathrm{~mm}, d=3 \mathrm{~mm})$ and large (H703-RB, $h=6 \mathrm{~mm}, d=6 \mathrm{~mm})$ magnets. The height $h$ and the diameter $d$ of the magnet were denoted in Figure 4-1(a). The magnetic flux density of the magnets on the glass surface was measured with a commercial gaussmeter (GM04, measurement range of 0.1 to $299.9 \mathrm{mT}$, error of $\pm 0.1 \mathrm{mT}$, Hirst Magnetics, United Kingdom). The measured flux densities on the surface and along the magnet axis were $38 \pm 0.1 \mathrm{mT}$ for the small magnet, $64 \pm 0.1 \mathrm{mT}$ for the medium magnet and $199 \pm 0.1 \mathrm{mT}$ for the large magnet, respectively. These values were the average density passing through the sensor area of the gaussmeter. Although the effect of the magnetic field was 3D (or 2D for our axis-symmetric case) and depended on the local flux density, the measured flux density was used here as the indication of the field strength of the different magnets. The ferrofluid droplets were dispensed using a micropipette (Finnpipette, Thermo Scientific, United States). This micropipette can dispense small volumes of liquid raging from $0.5 \mu L$ to $10 \mu L$. 
Droplets with different volumes were investigated. Since we intended to investigate the scaling relationship based on the base diameter of the droplet with a Bond number below unity, the volumes were selected to have base diameters ranging from $1 \mathrm{~mm}$ to $3 \mathrm{~mm}$. The droplets with different sizes were denoted as type $\mathrm{I}$ to type $\mathrm{V}$ in Table 4-1.

Table 4-1 Droplets with different sizes under investigation.

\begin{tabular}{ccc}
\hline droplet type & volume $(\mu \mathrm{L})$ & base diameter $(\mathrm{mm})$ \\
\hline I & 0.7 & $1.15 \pm 0.06$ \\
II & 1.3 & $1.57 \pm 0.08$ \\
III & 2.3 & $1.97 \pm 0.10$ \\
IV & 3.4 & $2.22 \pm 0.11$ \\
V & 4.8 & $2.61 \pm 0.13$
\end{tabular}

Table 4-2 Frame rates used for the different speeds of the magnets.

\begin{tabular}{ccc}
\hline speed $(\mathrm{mm} / \mathrm{s})$ & frame rate $(\mathrm{fps})$ & relative error $\varepsilon(\%)$ \\
\hline 0.2 & 2 & 30 \\
0.4 & 2 & 15 \\
0.6 & 3 & 15 \\
0.8 & 4 & 15 \\
1.0 & 7 & 21 \\
1.2 & 9 & 23 \\
1.4 & 11 & 24 \\
1.6 & 12 & 23 \\
1.8 & 13 & 22 \\
2.0 & 14 & 21
\end{tabular}

Figure 4-1(b) shows the schematic setup of the imaging system used in the experiments. The images of the ferrofluid droplet are captured by a CCD camera (Pulnix, progressive scan camera, JAI Inc., Japan). The camera software (Video Savant 3.0, IO Industries, Ontario, Canada) can record a series of 149 images frames with a given frame rate. Since the system has a zoom objective, the size of the image frame 
can be adjusted accordingly. When the objective is zoomed out to its limit, the largest error caused by pixel resolution is $30 \mu \mathrm{m}$. In each experiment, the frame rate was adjusted to match the velocity of the magnet (Table 4-2). The frames were stored as images or a single movie file. To observe the side view of the droplet, a $45^{\circ}$ mirror was placed next to the droplet. With the mirror, the camera could capture both top view and side view of the ferrofluid in a single frame. As mentioned above, a plastic ruler was placed under the glass slide to give a reference for the later evaluation of the captured images. The shape of the ferrofluid droplet was measured automatically using a customized MATLAB program. The program converted the recorded greyscale image into a binary image for further processing. In the stationary case, the image of the droplet was evaluated to extract parameters such as contact angle, base diameter, droplet height and radius of curvature. The data were evaluated on the basis of ten recorded frames. The experimental error is taken as the standard deviation of the collected data. The random error is caused by the lighting condition and pixel resolution when the image is converted from greyscale format to binary format. In the case of a moving droplet, the images were processed to extract the droplet velocity, the advancing and the receding contact angle. With the known frame rate, these parameters can be plotted as functions of time. The propagation of systems error from the measurement of displacement and the frame rate can be estimated as follows,

$$
\Delta v=\left|\frac{\Delta \delta_{x}}{\delta_{t}}\right|+\left|\frac{\delta_{x}}{\delta_{t}^{2}} \Delta \delta_{t}\right|
$$

where $\Delta v$ is the error of the evaluated velocity, $\delta_{x}$ is the displacement of the centroid of the droplet from one frame to the next frame, $\delta_{t}$ is the time delay between two 
frames, $\Delta \delta_{x}$ is the systematic error caused by camera resolution, and $\Delta \delta_{t}$ is the systematic error caused by the timer of the frame grabber. For the worst-case estimation of the systematic error of the evaluated velocity, the displacement, the special error and the time error can be estimated as $\delta_{x}=v \delta_{t}, \Delta \delta_{x}=0.03 \mathrm{~mm}$, and $\Delta \delta_{t}=10^{-6} \mathrm{~s}$. The relative systematic error of the velocity can then be calculated as $\varepsilon=\Delta v / v \times 100 \%$ and listed in Table $4-2$ for the different velocity and frame rates used in the later experiments.

The contact angles were evaluated by first extracting the droplet shape from the image, and then, a fifth-order polynomial was fitted to the droplet shape. The derivative of the fitting polynomial was calculated and used to determine the tangent and the corresponding contact angles at the two ends of the droplet. Since the coefficients of the polynomial might change in the recorded frames, it was difficult to derive a relative systematic error for the contact angles. In this study, relative random error was used for the contact angles. The random error was evaluated on the basis of polynomial fitting lines of the data and plotted as a confidence band. The random error of the evaluated contact angle was about $4 \%$. Because of smaller uncertainty in lighting condition and grey scale to binary conversion, a stationary droplet had a smaller random error for the contact angles. The random error propagation could be up to $25 \%$.

The images are cablibrated with the ruler scale captured in the experiment. Systematic error caused by blurry image is automatically corrected. The optical error is analyzed from a blur image. The image projection through a single lens is presented 
in Figure 4-2. The original point can become a blur spot due to the defocusing effect, which means that the point does not locate on the focus plane accurately. The blur spot diameter can be evaluated via,

$$
S_{d}=\frac{f_{x} c}{N_{f}} \frac{x_{d}}{x_{S} \pm x_{d}}
$$

where $S_{d}$ is the blur spot diameter, $f_{x}$ is the optical focal length, $c$ is the magnification of the optical system, $N_{f}$ is the lens f-number, $x_{S}$ is the distance from the focal plane to the lens and $x_{d}$ is the distance between the subject and the focus plane. During the adjustment of focus plane, the optical system cannot focus on the edge of the droplet precisely. But the edge is still within the depth of filed (DOF) to maintain the sharpness of the edge. Here, the analysis of error by blur spot is carried out by considering the worst case, $x_{d}=D O F / 2$. For the specific optical system used in our experiment, the DOF value and the distance from the focal plane to the lens $x_{S}$ are $0.12 \mathrm{~mm}$ and $89 \mathrm{~mm}$ respectively. The numerical aperture $N_{A}$ is 0.07 which makes the lens f-number $N \approx 1 /\left(2 N_{A}\right)=7.14$. Taking the value of $c=4.5$, the optical focal length $f_{x}$ is calculated to be $f_{x}=c x_{S} /(1+c)=72.82 \mathrm{~mm}$. Thus, the corresponding blur spot diameter is $0.031 \mathrm{~mm}$. The droplet diameter is around $1.9 \mathrm{~mm}$ and the relative error is calculated to be $0.031 /(1.9 / 2) \times 100 \%=3.3 \%<5 \%$. Therefore, the error caused by the blur image is negligible. 


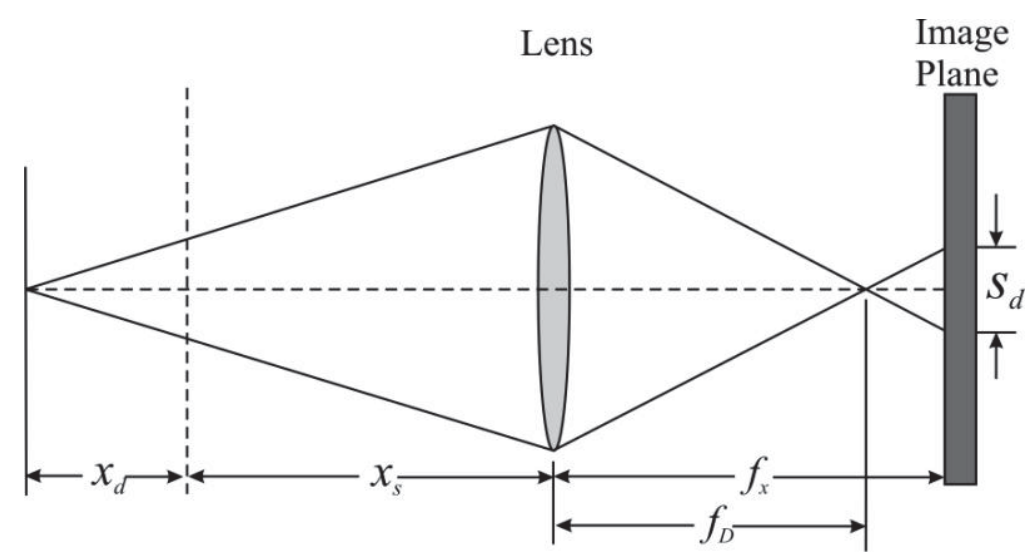

Figure 4-2 Image projection through a single lens.

\subsection{Results and Discussion}

The experimental results were analyzed corresponding to the case of modified wetting and sliding of sessile ferrofluid droplet under a nonuniform magnetic field. For stationary droplets, the important parameters, such as height, base diameter and contact angle, were investigated as functions of the magnetic flux density. In the study of droplet kinematics, the efforts were focused on the droplet position, velocity, advancing and receding contact angle. 


\subsubsection{Magnetowetting}

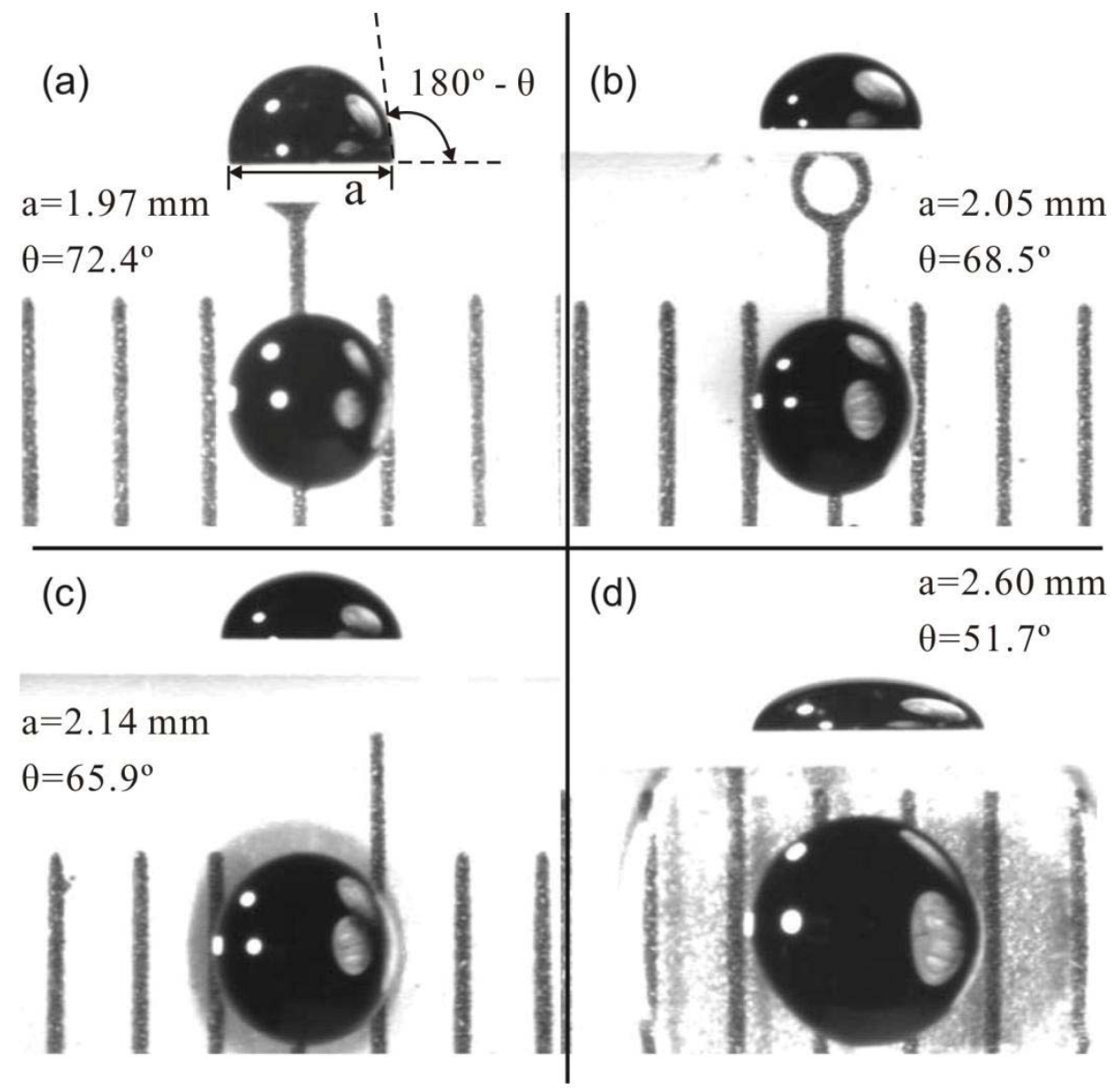

Figure 4-3 Sessile ferrofluid droplet (droplet III, V=2.3 $\mu \mathrm{L}$ ) under different magnetic field strengths: (a) no magnet (b) small magnet, $\mathrm{B}=38 \pm 0.1 \mathrm{mT}$ (c) medium magnet, $\mathrm{B}=64 \pm 0.1 \mathrm{mT}$ (d) large magnet, $\mathrm{B}=199 \pm 0.1 \mathrm{mT}$.

The investigation is first carried out to examine the effect of magnetic field strength on the shape and contact angle of a sessile ferrofluid droplet. This phenomenon is called here magnetowetting. Figure 4-3 shows the shape of droplet III (refer to Table 4-1) under different magnetic conditions. Without the presence of a permanent magnet, the droplet has a base diameter of $\mathrm{a}=1.97 \pm 0.10 \mathrm{~mm}$ and a static contact angle of $\theta=72.4 \pm 0.2^{\circ}$ (Figure 4-3(a)). Under a magnetic field in small and medium strength, the droplet is flattened and the contact angle decreases (Figure 4-3(b,c)). With the flux density of $199 \pm 0.1 \mathrm{mT}$ of the large magnet, the droplet takes the mostly flattened 
shape shown in Figure 4-3(d). The experiments were carried out many times by the same person using the same instrument, following the same procedure, locating at the same place within a short period of time. The repeatability of the experiment is analyzed by investigating droplet (V) height with field strength at 0.038 Tesla. The repeatability is the relative standard deviation which is calculated to be $0.063 / 1.18 \times 100 \%=5.3 \%$.
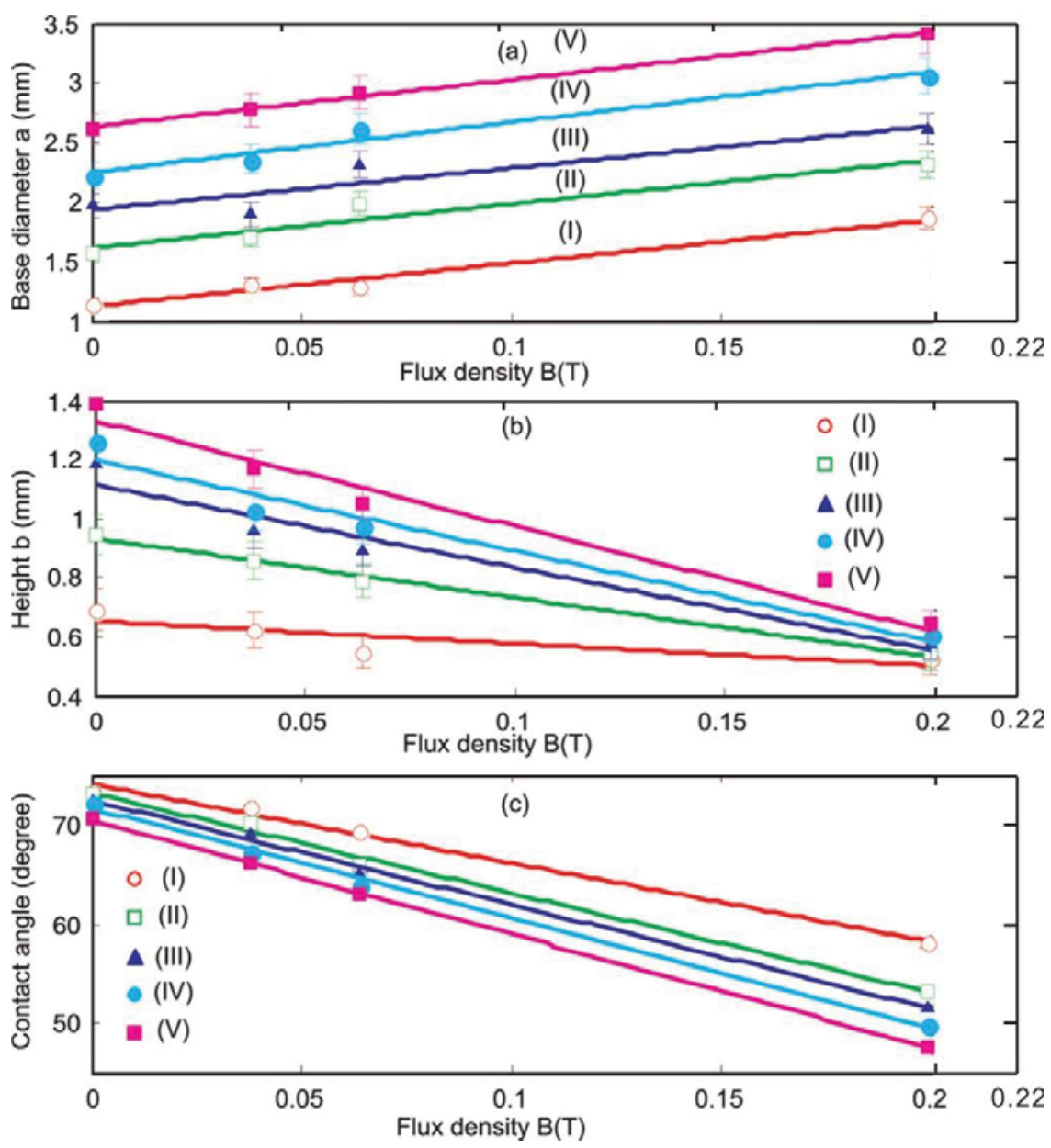

Figure 4-4 Magnetowetting effect of stationary ferrofluid droplets of different sizes (the lines are second-order fitting curves): (a) Base diameter (b) Droplet height (c) Apparent contact angle.

In general, a stronger magnetic field would pull down and laterally stretch the ferrofluid droplet, increasing its base diameter, decreasing its height and decreasing its 
apparent contact angle. Figure 4-4 shows the measured geometric parameters of the different stationary ferrofluid droplets as functions of the flux density. The base diameter of the ferrofluid droplet increases almost linearly with the flux density (Figure 4-4(a)). The height and contact angle of the ferrofluid droplet decrease almost linearly with the flux density (Figure $4-4(b, c)$ ). The dimensionless value of droplet height is defined as the ratio between the droplet height and the initial value. The variation of droplet height in Figure 4-4(b) is also analyzed with dimensionless value varies with the magnetic Bond number Eq. (3.35). From the trend shown in Figure 4-5, it is worth noting that its height varies exponentially with the magnetic Bond number.

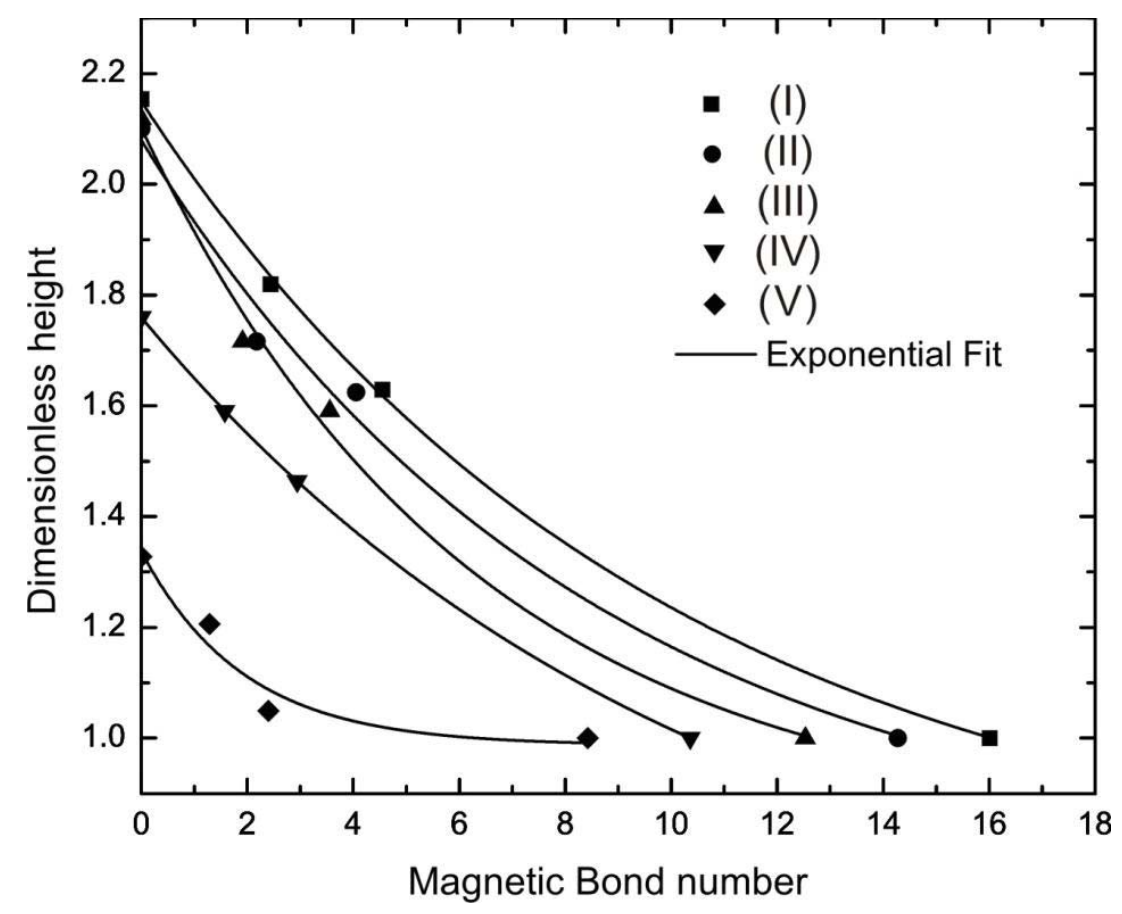

Figure 4-5 Dimensionless height varies with the magnetic Bond number. 


\subsubsection{Sliding of a Sessile Ferrofluid Droplet (Droplet Kinematics)}

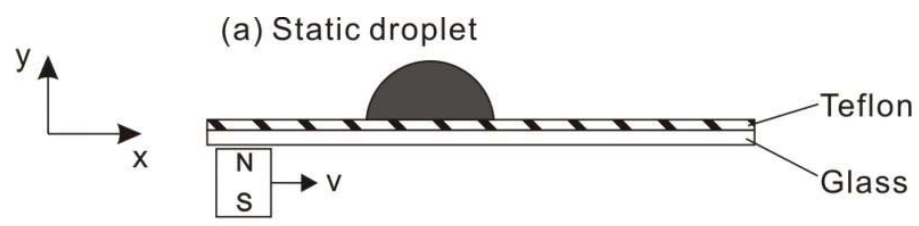

(b) Pinch off moment

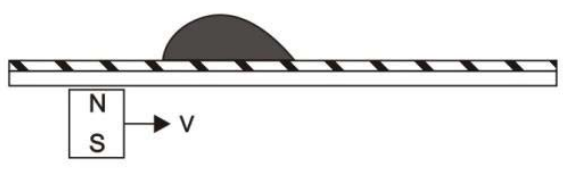

(c) Trapped droplet

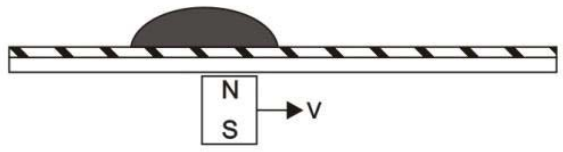

(d) Droplet with force balance

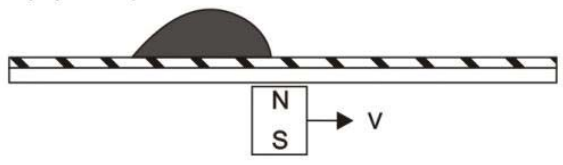

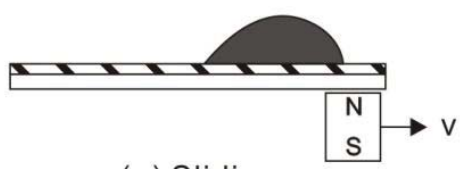

(e) Sliding

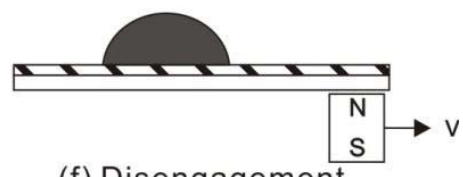

(f) Disengagement

Figure 4-6 Experimental procedure of a sessile ferrofluid droplet motion driven by a permanent magnet: (a) Droplet remains stationary and the magnet starts to move; (b) Pinch off moment; (c) Droplet keeps deform upon been trapped by the magnet; (d) Droplet deforms o the shape with force balance on it; (e) Sliding; (f) Disengagement.

Without the presence of a magnetic dragging force, a ferrofluid droplet remained in a stationary condition (Figure 4-6(a)). When a permanent magnet approached from the left, the ferrofluid droplet would deform to achieve the pinching-off state (Figure 4-6(b)). After being trapped by the magnet (Figure 4-6(c)), the droplet would continue to deform with the change of distance between the droplet and the magnet. After achieving the shape with force equilibrium (Figure 4-6(d)), the ferrofluid droplet would be dragged to slide for a certain distance with the magnet. Depending on velocity of the magnet, two situations would occur: steady droplet sliding (Figure 
4-6(e)) and disengagement (Figure 4-6(f)). In the experiments, three parameters involved in this motion were varied: three different magnets (small, medium and large), five different droplet base diameters (Table 4-1), and ten different magnet velocities ranging from $0.2 \mathrm{~mm} / \mathrm{s}$ to $2 \mathrm{~mm} / \mathrm{s}$. Two basic operating regimes were observed: sliding (Figure 4-7) and disengagement (Figure 4-8).
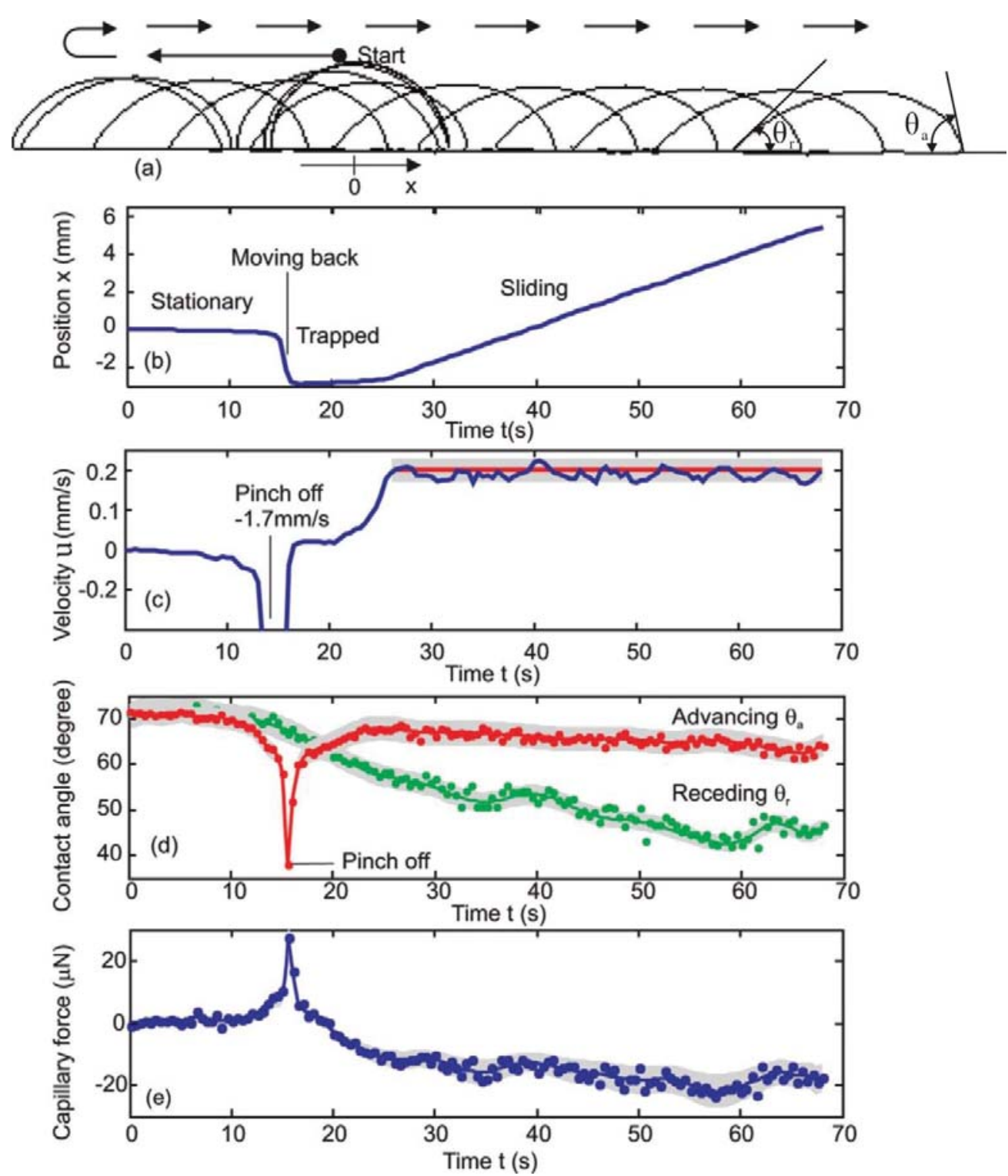

Figure 4-7 Sliding of a sessile ferrofluid droplet in the presence of a moving permanent magnet ( $\mathrm{h}=2 \mathrm{~mm}, \mathrm{~d}=3 \mathrm{~mm}, \mathrm{~B}=64 \mathrm{mT}, \mathrm{u}=0.2 \mathrm{~mm} / \mathrm{s}$ and $\mathrm{a} \approx 2.22 \mathrm{~mm}$ ): (a) Evolution of the tracked droplet (every 10 frames); (b) Displacement of the droplet centroid versus time; (c) Velocity of the droplet versus time. The constant velocity of $0.2 \mathrm{~mm} / \mathrm{s}$ is plotted as the red line with a confidence band of $\pm 30 \%$; (d) Contact angles versus time. The confidence interval is $\pm 4 \%$; (e) Capillary force versus time. The confidence interval is $\pm 25 \%$. 
If the magnet speed is slow enough and the magnetic force is large enough (strong magnet or large droplet), the ferrofluid droplet can slide with the same velocity as the magnet. Figure 4-7 shows the typical behaviour of a ferrofluid droplet in sliding regime. Without the magnet, the stationary droplet has a contact angle of $72^{\circ}$. Figure 4-7(a) shows the time evolving image of the ferrofluid droplet IV with an initial base diameter of $2.22 \mathrm{~mm}$, moving with the medium magnet from the left at a velocity of $0.2 \mathrm{~mm} / \mathrm{s}$. The approaching magnet pulls the droplet to the left. The droplet deforms so that the right contact angle (the later advancing contact angle $\theta_{a}$, red line in Figure 4-7(d)) decreases significantly. Since the droplet is still stationary, only the capillary force resists the magnetic force. At the pinching moment ( $\approx 12 \mathrm{~s}$ in Figure 4-7(d)), the solid/liquid/gas contact line is displaced and the right and left contact angles drop to $\theta_{a}=38^{\circ}$ and $\theta_{r}=67^{\circ}$, respectively. The capillary force and the magnetic force can be estimated for this moment as $F_{m}=F_{c a p}=a \sigma\left(\cos \theta_{a}-\cos \theta_{r}\right) \approx 28 \mu N$. Figure 4-7(e) shows the capillary force as a function of time. To appreciate the magnitude of the magnetic force, the weight of the droplet calculated with the given volume $V=3.4 \mu \mathrm{L}$ and density $\rho_{F F}=1.07 \times 10^{3} \mathrm{~kg} / \mathrm{m}^{3}$ is $3.64 \mu \mathrm{N}$. From the pinching moment, the droplet accelerates to $1.7 \mathrm{~mm} / \mathrm{s}$ as shown in Figure 4-7(c). After being trapped to the magnet ( $\mathrm{t}>13 \mathrm{~s}$ in Figure 4-7(b)), the droplet continues to deform and accelerates to the steady velocity of the magnet ( $>13 \mathrm{~s}$ in Figure 4-7(c)). In this process, the relative distance between the magnet and the droplet is adjusted to reach equilibrium between capillary force, friction force and magnetic force. The evaluated velocity in Figure 4-7(c) shows a wavy characteristic, which is caused by the stepper motor driving the 
linear stage. The advancing contact angle then reaches a steady-state value of approximately $64^{\circ}$ (red line in Figure 4-7(d)), while the receding contact angle approaches a value of approximately $46^{\circ}$ (green line in Figure 4-7(d)). The capillary force in the steady-state sliding motion remains constant at a value of $F_{c a p} \approx 18 \mu N$ (Figure 4-7(e)).

With the same condition as the previous experiment (Figure 4-7), the droplet cannot move if the magnet velocity is higher than $1.6 \mathrm{~m} / \mathrm{s}$. Figure $4-8$ shows the behaviour of the droplet at $\mathrm{u}=1.8 \mathrm{~m} / \mathrm{s}$. Similar to the previous case, the droplet was pinched off by the approaching magnet. With $\theta_{a}=61^{\circ}$ and $\theta_{r}=69^{\circ}$, the magnetic force at the pinching moment is approximately $F_{m}=F_{c a p} \approx 9 \mu N$ (Figure 4-8(e)). This force is smaller than in the previous case because of the higher approaching velocity, and the exact pinching moment cannot be captured. The droplet first accelerates to reach the steady velocity of the magnet, where the disengagement occurs because the velocity subsequently drops. At this disengagement moment (Figure 4-8(c)), the contact angles were measured as $\theta_{a}=69^{\circ}$ and $\theta_{r}=60^{\circ}$. The corresponding capillary force is $F_{c a p} \approx 10 \mu N$. Subsequently, the receding contact angle reaches a minimum value of $\theta_{r}=58^{\circ}$, where the advancing contact angle is $\theta_{a}=70^{\circ}$. The corresponding capillary force at this moment is $F_{c a p} \approx 13 \mu N$. After becoming stationary again, the contact angle on both side of the droplet reaches $67^{\circ}$, dropping from the initial $73^{\circ}$. 
(a)
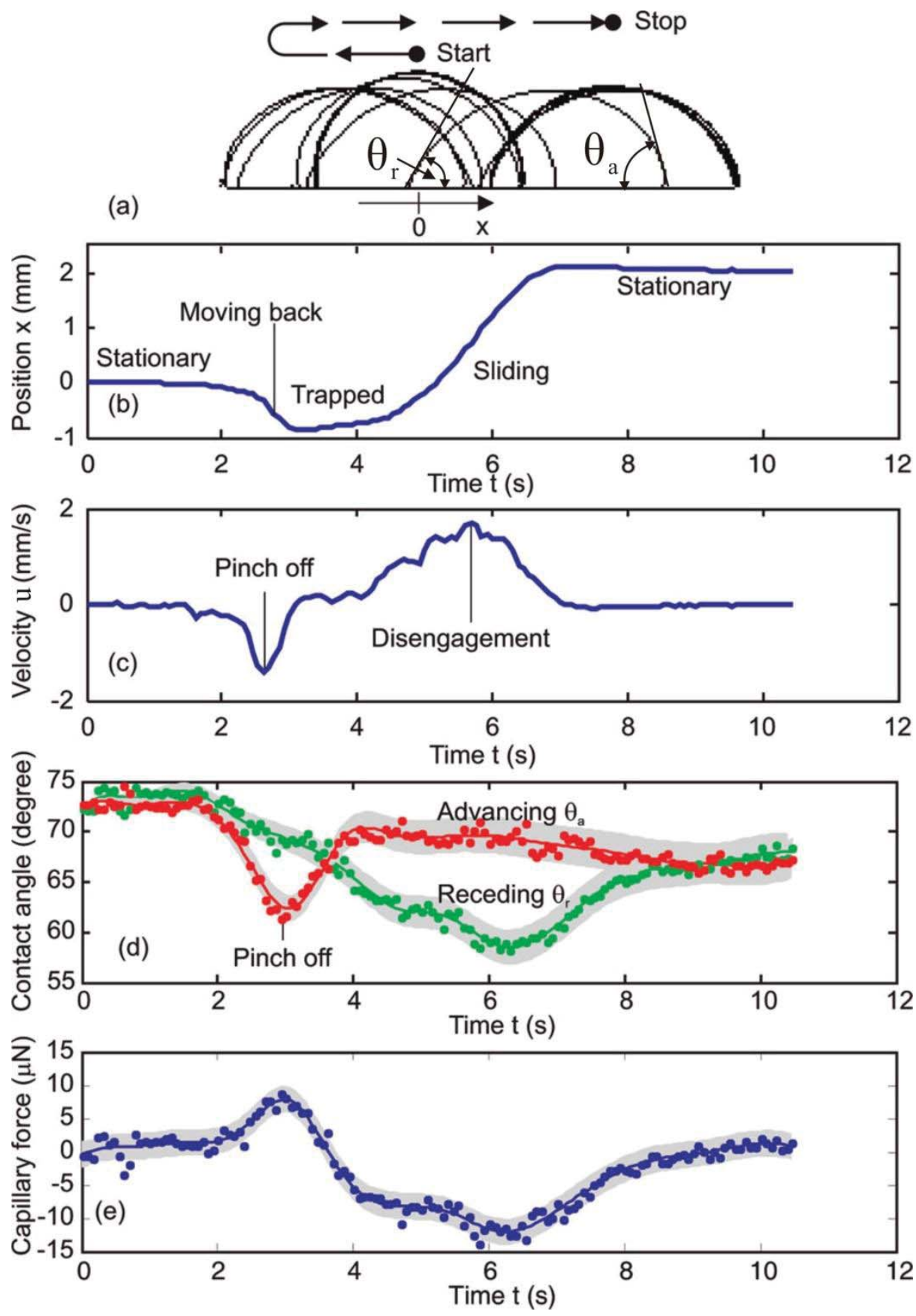

Figure 4-8 Disengagement of a sessile ferrofluid droplet in the presence of a fast moving permanent magnet $(\mathrm{h}=2 \mathrm{~mm}, \mathrm{~d}=3 \mathrm{~mm}, \mathrm{~B}=64 \mathrm{mT}, \mathrm{u}=1.8 \mathrm{~mm} / \mathrm{s}$ and $\mathrm{a} \approx 2.22 \mathrm{~mm}$ ): (a) Evolution of the tracked droplet (every 10 image frames); (b) Displacement of the droplet centroid versus time; (c) Velocity of the droplet versus time; (d) Contact angles versus time. The confidence interval is $\pm 4 \%$; (e) Capillary force versus time. The confidence interval is $\pm 25 \%$.

Next, droplet kinematics was investigated for its dependence on the magnetic strength, base diameter and velocity of the moving magnet. Figure 4-9(a) compares the behaviour of the smallest droplet I (Table 4-1) affected by three different magnets 
moving at the same velocity of $0.2 \mathrm{~mm} / \mathrm{s}$. The small magnet can pinch off the droplet and pull it back. However, the magnetic force is not large enough to return the droplet to its original position. As the small magnet moves away, the droplet is positioned 100 $\mu \mathrm{m}$ left of the initial position. The contact angle reduces from the initial value of $73^{\circ}$ to $68^{\circ}$. The same behaviour was observed with the medium magnet. However, the magnet is strong enough to drag the droplet forward, so that after the disengagement the droplet is positioned $200 \mu \mathrm{m}$ right of the initial position. The large magnet is strong enough to drag the droplet along. The behaviour is similar to that described in Figure 4-7. The strength of the magnetic force is correlated well with the trapping position.
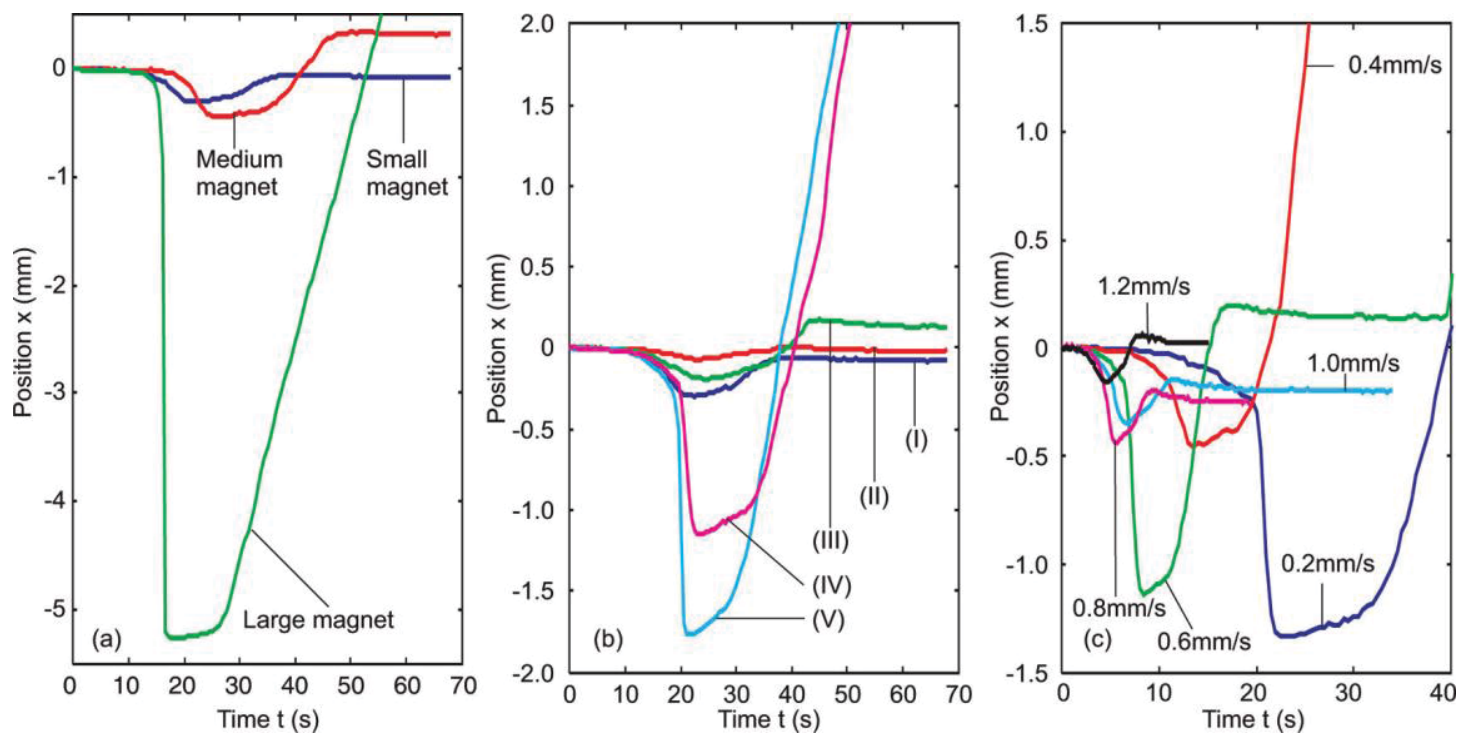

Figure 4-9 The dependence of droplet kinematics on different parameters: (a) Magnetic strength (droplet I, magnetic velocity of $0.2 \mathrm{~mm} / \mathrm{s}$ ); (b) Base diameter of droplet (medium magnet, magnet velocity of $0.2 \mathrm{~mm} / \mathrm{s}$ ); (c) Velocity of the magnet (medium magnet, droplet II).

Figure 4-9(b) shows the kinematic behaviour of droplets with different sizes. The droplets are dragged by the medium magnet at $0.2 \mathrm{~mm} / \mathrm{s}$. Similar to the case of different magnets, the magnetic force depends on the size of the droplet. Small droplets have a weak magnetic force and consequently cannot slide along with the 
magnet. Besides the magnetic force, the base diameter also affects the friction force that in turns affects the behaviour of the droplet displacement. With an optimal size (droplet II), the magnetic force is balanced by the friction force minimizing the displacement of the droplet. Figure 4-9(b) shows that droplet II has both smallest displacement and trapping distance. As discussed later by the scaling analysis, the magnetic force scales with the third order of the base diameter and the friction force with the second order of the base diameter. Large droplets make the magnetic force strong enough to overcome initial friction and to slide into motion. In the case of droplet IV, the magnetic force just reaches the threshold causing the sliding velocity to fluctuate due to the readjustment of the relative distance between the droplet and the magnet. Droplet $\mathrm{V}$ is large enough so that the distance and consequently the magnetic force are steady during the sliding motion.

Figure 4-9(c) shows the behaviour of droplet II dragged by the medium magnet at different velocities. The kinematical behaviour clearly shows the effect of the friction force, which is proportional to the velocity of the droplet. At $0.2 \mathrm{~mm} / \mathrm{s}$ and $0.4 \mathrm{~mm} / \mathrm{s}$, the droplet is still able to follow the magnet. At velocities higher than $0.6 \mathrm{~mm} / \mathrm{s}$, the friction force is so large that disengagement occurs. The following scaling analysis discusses the relationship among the velocity, the base diameter, and the sliding regimes in more details. 


\subsubsection{Scaling Analysis and Operating Map}

A scaling analysis has been conducted based on the kinematic behaviour of a ferrofluid droplet under various driving conditions. The kinematic behaviour of the ferrofluid droplet depicted in Figure 4-9 indicates the three parameters determine the operating regime of a sliding ferrofluid droplet: the magnetic field strength, the size of the droplet represented by its base diameter, and the velocity of the magnet. Since the magnetic field strength is adjusted in the droplet/surface/magnet system by the distance between the magnet and the droplet, the two key parameters for the scaling relationships and the operating map for the sliding ferrofluid droplet are the base diameter $a$ and the magnet velocity $u$.

As shown in Figure 4-1(a), the forces balance in the $x$-axis of a sliding ferrofluid droplet is,

$$
F_{m}=F_{f}+F_{c a p}
$$

where $F_{m}$ is the driving magnetic force, $F_{f}$ is the friction force, and $F_{c a p}$ is the capillary force caused by the difference between advancing and receding contact angles. For a constant and uniform field gradient across the droplet, the magnetic force is determined by,

$$
F_{m}=\frac{V \chi_{m}}{\mu_{0}} B \nabla B=\alpha a^{3}
$$

where $V \propto a^{3}$ is the volume of the droplet, $\chi_{m}$ is the magnetic susceptibility of the ferrofluid, $\mu_{0}$ is the permittivity of free space, and $B$ is the magnetic flux density applied on the droplet. With a fixed relative position between the droplet and the 
magnet, factor $\alpha$ represents the strength of the magnetic field. The scaling relationship of the magnetic force is $F_{m} \propto a^{3}$.

The friction force acting against the sliding motion of the droplet can be estimated as,

$$
F_{f}=K_{f} A \eta_{F F} u=\beta a^{2} u
$$

where $K_{f}$ is the friction factor, $A=\pi a^{2} / 4$ is the contact area, $\eta_{F F}$ is the viscosity of the ferrofluid, and $u$ is the droplet velocity relative to the surface. The relationship in Eq. (4.5) considers the flattened shape of the droplet due to the strong magnetic pull, where the factor $K_{f}$ is assumed to be constant. The scaling relationship of the friction force is $F_{f} \propto a^{2}$. The factor $\beta$ in Eq. (4.5) represents the friction between the droplet and the solid surface.

The capillary force acting against the sliding motion is,

$$
F_{c a p}=a \sigma\left(\cos \theta_{r}-\cos \theta_{a}\right)=\gamma a
$$

where $\sigma$ is the surface tension of the ferrofluid. Since the advancing and receding contact angles, $\theta_{a}$ and $\theta_{r}$, remain constant in the steady-state sliding condition. The factor $\gamma$ represents the strength of capillary. The scaling relationship of the capillary force is therefore $F_{c a p} \propto a$.

The droplet can slide if the velocity of magnet is less than or equal to a critical value. For the magnet moving with a critical velocity $u_{c r}$, the force balance on the droplet been dragged has the form,

$$
\alpha a^{3}=\beta a^{2} u_{c r}+\gamma a
$$


Rearranging the above equation leads to the scaling relationship between the critical velocity and the base diameter of the droplet,

$$
u_{c r}=\frac{\alpha}{\beta} a-\frac{\gamma}{\beta} \frac{1}{a}
$$
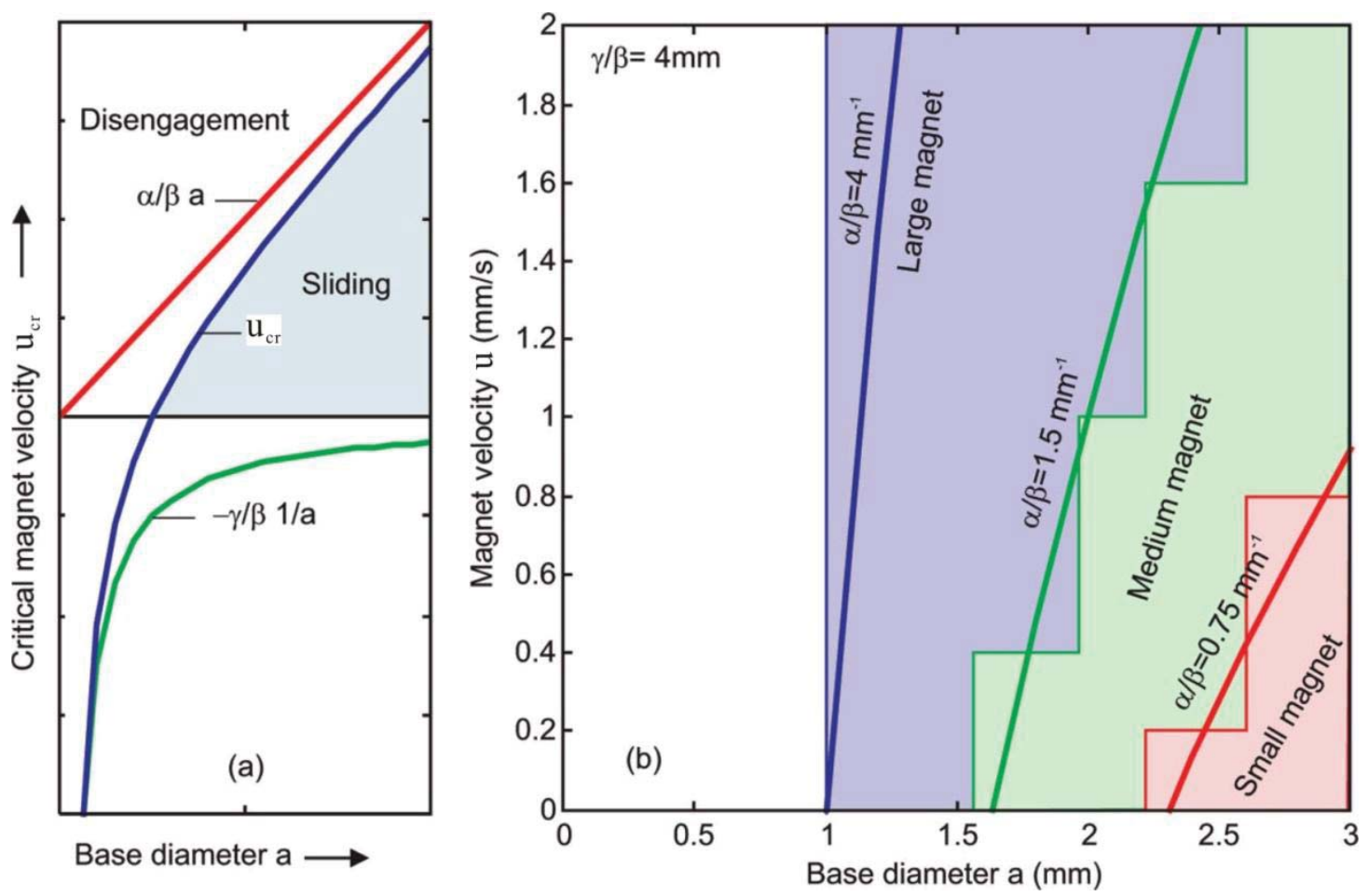

Figure 4-10 Operating map of a sliding ferrofluid droplet: (a) Scaling law for the critical velocity. (b) Operating map of the ferrofluid droplets in our experiments $\left(\beta \approx 2 \mu N s / \mathrm{mm}^{3}, \gamma \approx 8\right.$ $\mu N / \mathrm{mm})$. The red-shaded area is the sliding regime with the small magnet $\left(\alpha \approx 1.5 \mu \mathrm{N} / \mathrm{mm}^{3}\right)$. The green-shaded area is the sliding region of the medium magnet $\left(\alpha \approx 3 \mu N / \mathrm{mm}^{3}\right)$. The sliding region of the large magnet is shaded in blue $\left(\alpha \approx 8 \mu \mathrm{N} / \mathrm{mm}^{3}\right)$.

Figure 4-10(a) depicts the two terms forming the curve of the critical velocity on the velocity/base diameter operating map. The shaded area to the right of the curve represents the sliding regime. In this region, the magnetic force is large enough to overcome the friction and to drag the droplet along. A low velocity and a large droplet would make sliding possible. Therefore, the sliding region occupies the lower righthand corner of the operating map. Figure 4-10(b) shows the operating map observed in 
the experiment. The red, green, and blue-shaded areas are the sliding regions of the small magnet, medium magnet, and large magnet, respectively.

The parameter $\alpha, \beta$, and $\gamma$ from the scaling analysis were used to fit the experimental data. To start with, data of the medium magnet and droplet IV (Figure 4-7) were used due to the relatively low noise of the data of the capillary force. The maximum capillary force of the pinch-off moment of $28 \mu N$ is used to estimate the magnetic force. From Eq. (4.4) and $a=2.22 \mathrm{~mm}$, the scaling factor for the magnetic force is determined as $\alpha=3 \mu \mathrm{N} / \mathrm{mm}^{3}$. From Eq. (4.6) and a capillary force during the sliding motion of $F_{c a p} \approx 18 \mu N$, the scaling factor for the capillary force is determined as $\gamma=8 \mu \mathrm{N} / \mathrm{mm}$. Since there is no further information for the estimation of the friction force, the values of $\alpha$ and $\gamma$ were used to find $\beta=2 \mu N \mathrm{~N} / \mathrm{mm}^{3}$ so that the curve of Eq. (4.8) can fit the experimental data of the medium magnet shown in Figure 4-10(b). Subsequently, the values of $\gamma=8 \mu N / m m$ and $\beta=2 \mu N s / \mathrm{mm}^{3}$ and Eq. (4.8) were used to find $\alpha \approx 1.5 \mu \mathrm{N} / \mathrm{mm}^{3}$ and $\alpha \approx 8 \mu \mathrm{N} / \mathrm{mm}^{3}$ that can fit the data of the small and the large magnet, respectively.

The above scaling analysis and fitting scaling factors only qualitatively explain the interactions among the forces involved in the sliding motion. In fact, only the magnitude of the capillary force can be extracted from the measurement. The magnetic force at the pinch-off moment is not necessarily the same as the magnetic force during the sliding motion, because the relative distance between the droplet and the magnet is changed with the speed of the magnet. Depending on the speed and the size of the magnet, the relative distance and the magnetic force can vary. The friction force 
depends on the gravitational force and also the y-component of the magnetic force. Since the y-component of the magnetic force can be 1 or 2 orders of magnitude larger than the weight of the droplet, the friction force is also dominated by the magnetic force, which in turn varies as mentioned above.

\subsection{Summary}

The effect of magnetowetting and the sliding motion of a sessile ferrofluid droplet have been investigated experimentally. The results show that the shape of a sessile ferrofluid droplet is deformed in the presence of a magnetic field. The deformation depends on the local magnetic field. Using permanent magnets, we are able to observe the basic features of magnetowetting phenomenon. A higher flux density leads to a larger base diameter and a smaller height. More importantly, a higher flux density reduces the contact angle at the solid/liquid/gas contact line. We also investigate the kinematic behaviour of the droplet dragged by the permanent magnet at a constant velocity. If the magnetic force is large enough to overcome the resistance of the friction force and the capillary force, the droplet can slide along with the same linear velocity as the magnet. This chapter describes the detailed kinematic behaviour of the droplet as well as the change of its contact angles. Contact angle hysteresis was observed from the droplet after being pinched off from its initial position and resuming the stationary condition. Scaling analysis is developed to describe the operation regions of the sliding ferrofluid droplets. On a velocity-versus-base-diameter operating map, the sliding region occupies the lower right-hand corner. The critical velocities 
derived from the scaling analysis and parameters fitted to experimental data describe this map well. A more detailed analytical model or a numerical model is needed to explain and to appreciate the phenomena observed in the experiments. Results presented on magnetowetting effect and magnetic manipulation of ferrofluid droplets in particular or magnetic droplets in general would have potential impacts on dropletbased microfluidics interface control of liquid lenses, and possibly e-paper technology. 


\section{CHAPTER 5}

\section{MAGNETOFLUIDIC SPREADING IN MICRO-}

\section{CHANNELS}

This chapter presents the investigation of the spreading phenomena caused by the interaction between a uniform magnetic field and a magnetic fluid in microchannels. The flow system consists of two liquids: a ferrofluid and a mineral oil. The ferrofluid consists of superparamagnetic nanoparticles suspended in an oil-based carrier. Under a uniform magnetic field, the superparamagnetic particles are polarized and represent magnetic dipoles. The magnetization of magnetic nanoparticles results in magnetic force acting on each particle, affecting the corresponding particle concentration distribution. Mixing due to secondary flow close to the interface also contributes to the spreading of the ferrofluid. Both experimental study and numerical simulation are conducted. The experimental setup and the results are presented and discussed in detail. The uniform magnetic field is generated by a custom-made electromagnet. The flow rate ratio between the mineral oil and oil-based ferrofluid is varied to test the spreading performance. The numerical model is developed and carried out to verify the experimental data. The mass, momentum and diffusion equations are solved by the Finite Volume Method (FVM). 


\subsection{Experimental Setup}

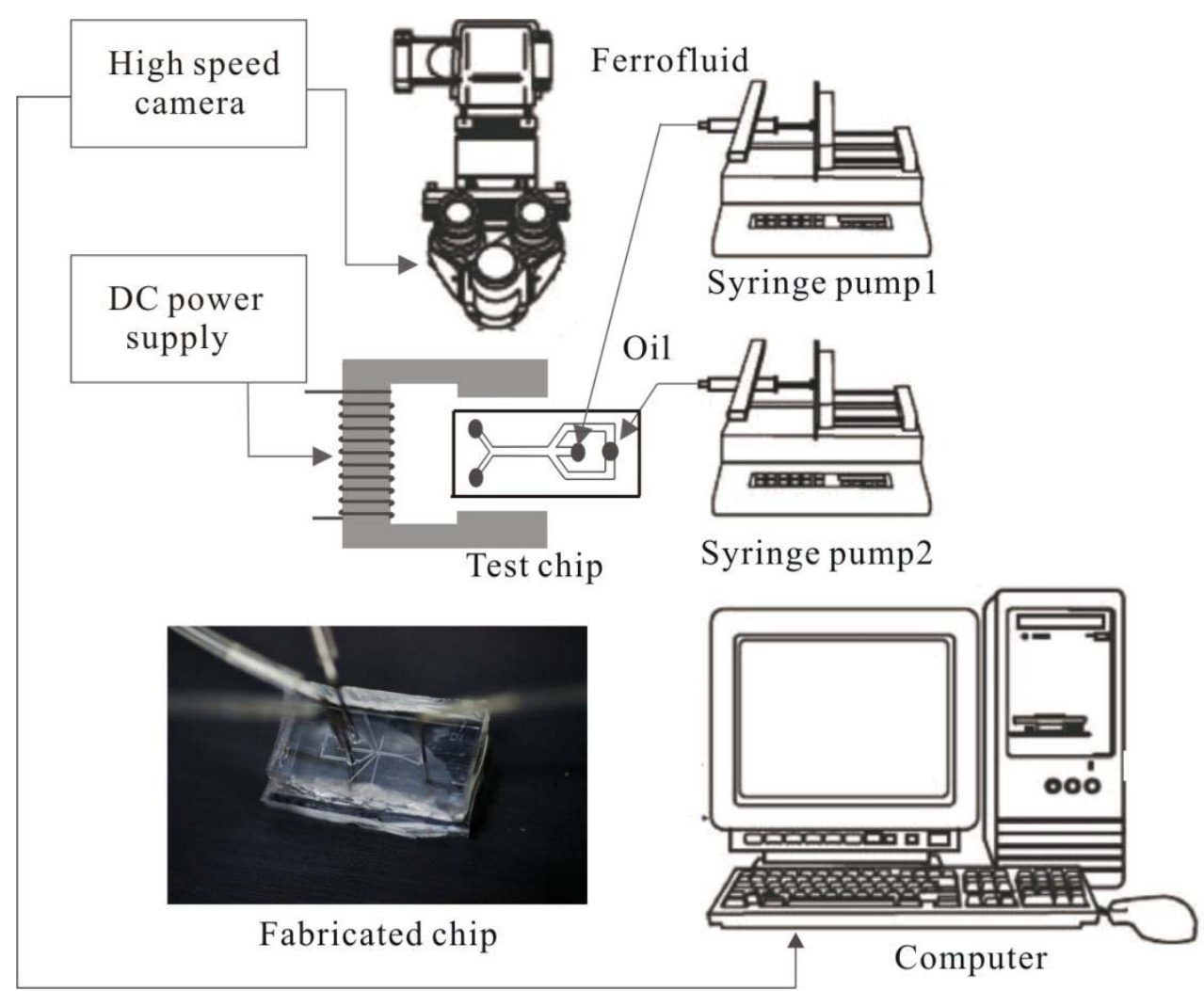

Figure 5-1 Experimental setup for magnetofluidic spreading in microchannels.

Figure 5-1 shows the experimental setup for the magnetofluidic spreading in microchannels. The microfluidic device was cut to fit into the air gap of the electromagnet. The fluids were delivered into the microfluidic device using two separated precision syringe pumps (KD Scientific Inc., USA). The whole setup was placed on a Nikon (Eclipse TE2000-E) inverted microscope equipped with a highspeed camera (Photron, APX RS). For generating the magnetic field, a current was supplied to the electromagnet by a DC power supply (GPS-3030D). The images of the flow system were captured for further analysis. 


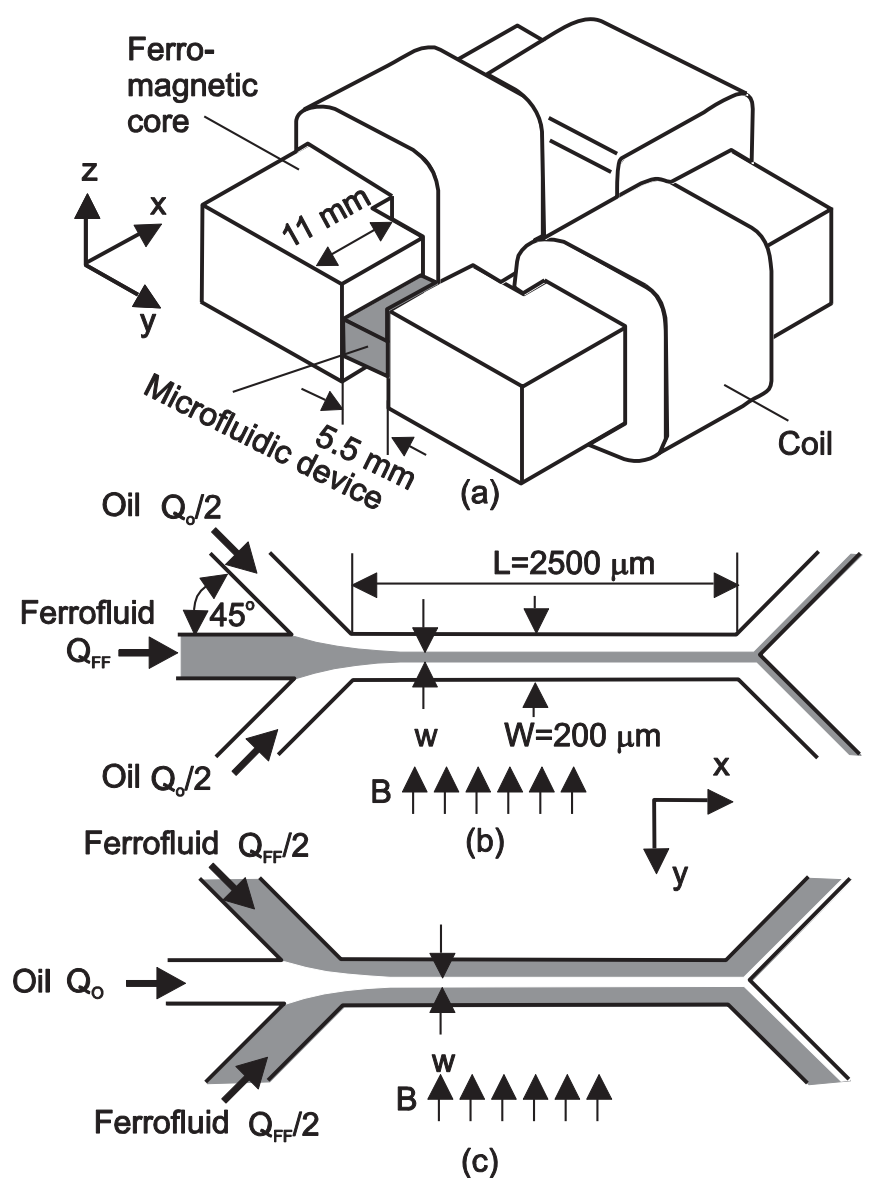

Figure 5-2 The field generation, channel and flow configuration for magnetofluidic spreading: (a) Electromagnet for generation of an uniform magnetic field; (b) Magnetofluidic spreading with ferrofluid-core configuration; (c) Magnetofluidic spreading with ferrofluid-cladding configuration.

The detailed information of field generation, channel and flow configuration are illustrated in Figure 5-2. The flow system consisted of two liquids: a ferrofluid and a mineral oil. The ferrofluid (APG 2134, FerroTec) was formed by superparamagnetic nanoparticles suspended in an oil-based carrier fluid. The ferrofluid had a saturation magnetization of $22 \mathrm{mT}$ and a density of $\rho_{F F}=1.07 \times 10^{3} \mathrm{~kg} / \mathrm{m}^{3}$. According to FerroTec, the ferrofluid is only slightly shear-dependent with the viscosity decreasing from $984 \mathrm{mPa} \cdot \mathrm{sec}$ to $967 \mathrm{mPa} \cdot \mathrm{sec}$ for a shear rate ranging from $46 \mathrm{sec}^{-1}$ to $196 \mathrm{sec}^{-1}$. The maximum increase in viscosity due to magnetoviscous effect is about 
$2 \%$ [205]. The mineral oil (Sigma-Aldrich, Cat No. 330779) has a viscosity of $\eta_{o}=23.6 \mathrm{mPa} \cdot \mathrm{sec}$ and a density of $\rho_{o}=0.838 \times 10^{3} \mathrm{~kg} / \mathrm{m}^{3}$. The zero-shear viscosity is given by the manufacturer as $\eta_{F F 0}=1000 \mathrm{mPa} \cdot \mathrm{sec}$. We modified a transformer to make the electromagnet, shown in Figure 5-2(a). An air gap of $5.5 \mathrm{~mm}$ was cut through the ferromagnetic core of the transformer to accommodate the microfluidic device. The length of the test section was $11 \mathrm{~mm}$. By varying the supply current up to 1.8 A, the electromagnet can generate a magnetic flux density up to $50 \mathrm{mT}$ in the air gap. The calibration of the custom-made electromagnet is presented in Figure 5-3. In Figure 5-4, the uniformity of the magnetic field is examined with current fixed at 0.2 A. The mass magnetization of ferrofluid APG 2134 is presented in Figure 5-5 with the variation of magnetic flux density in air. The magnetization data is obtained from the manufacturer.

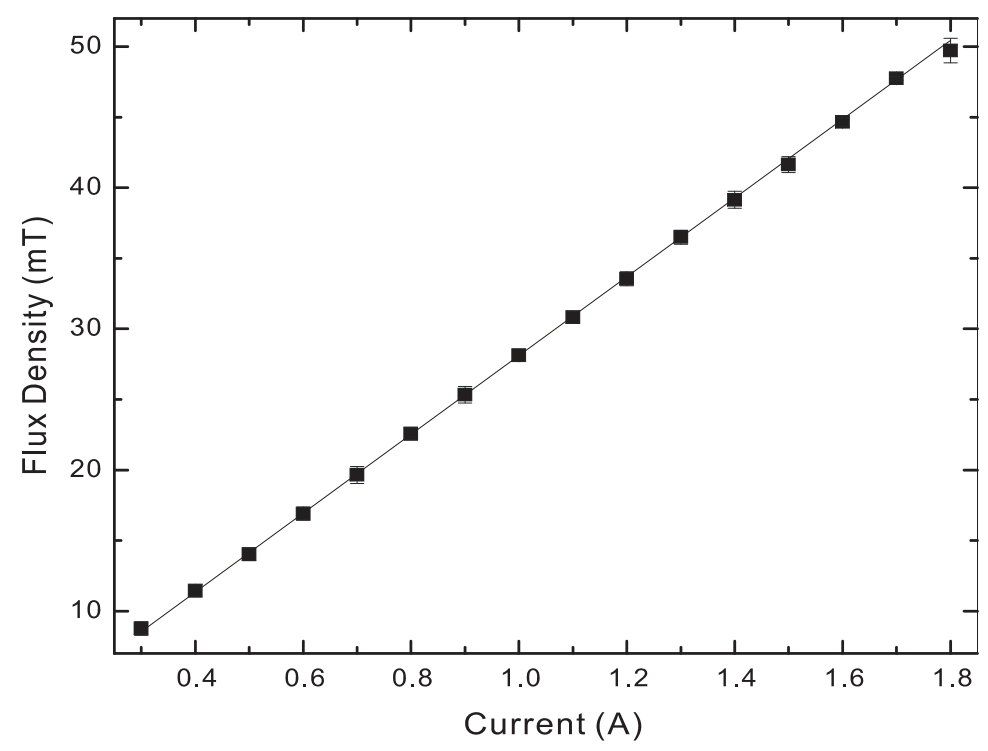

Figure 5-3 The calibration of the custom-made electromagnet with flux density up to $50 \mathrm{mT}$. 


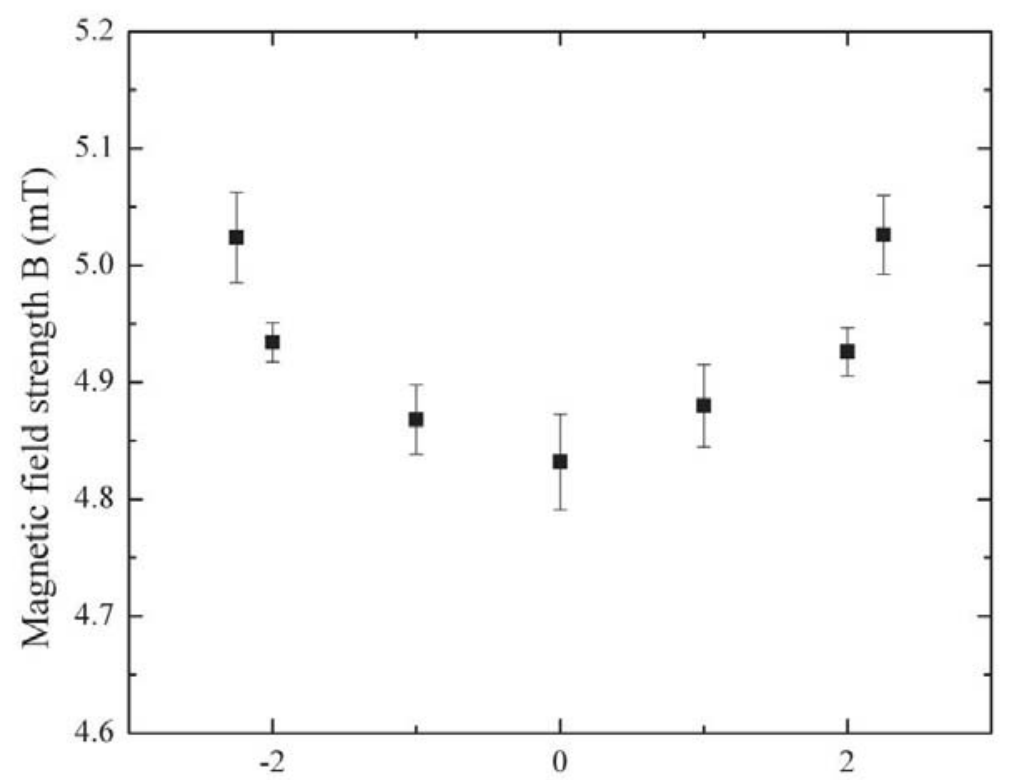

Position in the air gap of the electromagnet $(\mathrm{mm})$

Figure 5-4 The variation of magnetic field strength in the air gap.

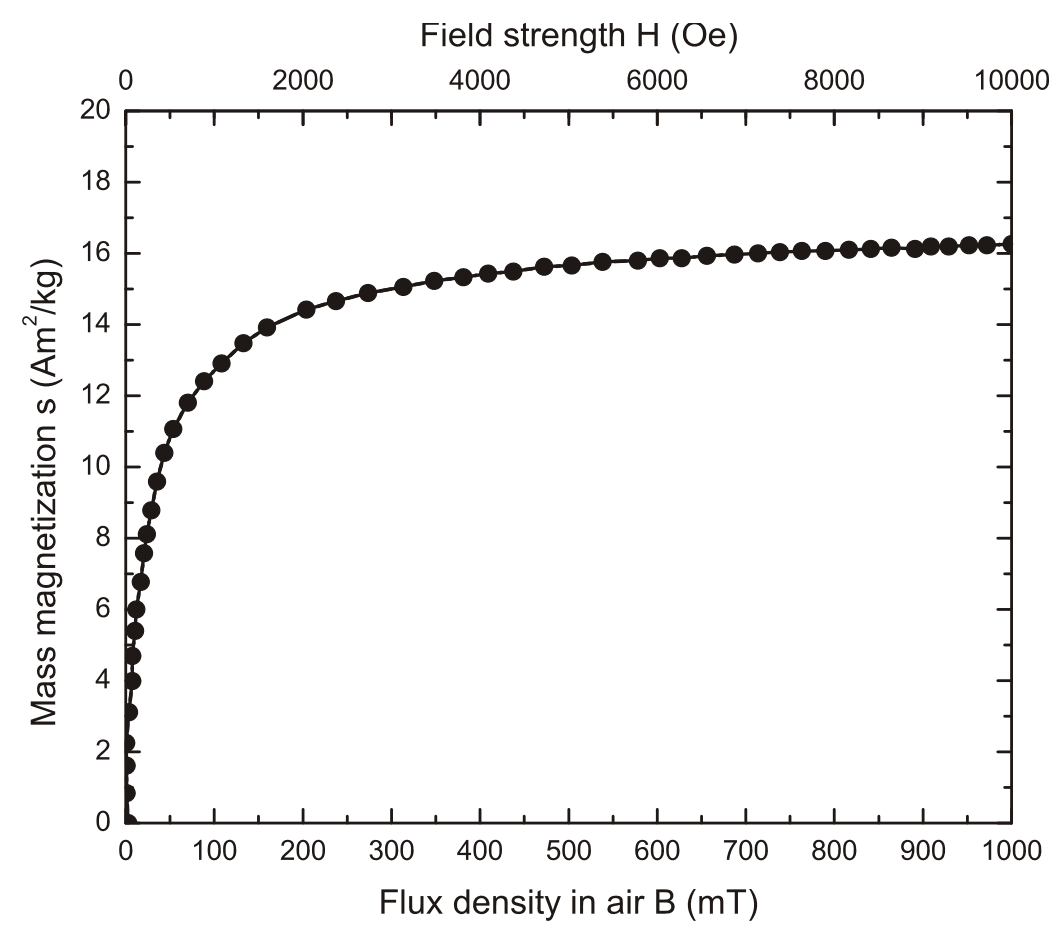

Figure 5-5 Mass magnetization of ferrofluid (APG 2134, FerroTec) at the corresponding magnetic flux density in the air.

The microfluidic device was made of polydimethylsiloxane (PDMS) by soft lithography. The test channel had three inlets and two exits. As shown in Figure 5-2(b) and (c), the test channel has a depth of $h=50 \mu \mathrm{m}$, a width of $w=200 \mu \mathrm{m}$ and a 
length of $l=2500 \mu \mathrm{m}$. The length of the test channel is less than $1 / 4$ of the width of the section in the air gap. Thus, a uniform magnetic field perpendicular to the microchannel can be assumed. With a size of magnetic particles of about $d_{\mathrm{p}}=10 \mathrm{~nm}$ and a temperature of $\mathrm{T}=300 \mathrm{~K}$, the diffusion coefficient of the particles into the mineral oil is estimated according to Einstein's model, $D_{f}=k_{B} T / 3 \pi \eta_{o} d_{p}=1.86 \times 10^{-12} \mathrm{~m}^{2} / \mathrm{s}$, where $k_{\mathrm{B}}$ is the Boltzmann constant. This channel configuration together with the magnetic field can also be used for droplet formation and controlling process. First, the core stream and the cladding stream are required to be immiscible for droplets generation. Second, the generation of droplet depends on the force balance among the interfacial tension, viscous force and the pressure driven force. As a result, the flow rate ratio and the channel configuration need to be optimized to generate a neck and make the neck to break easily to form a droplet. It is worth noting that the magnetic force can only affect the formation process but cannot form a droplet by itself.

In the spreading experiment with ferrofluid-core configuration [Figure 5-2(b)], the ferrofluid was delivered into the middle inlet at a constant flow rate $Q_{F F}$ of $0.05 \mathrm{ml} / \mathrm{h}$. The flow rate of the two sheath streams $Q_{o}$ was varied from 0.2 to $0.6 \mathrm{ml} / \mathrm{h}$. Using the properties of the mineral oil, the Reynolds number $\operatorname{Re}=\rho_{o} U D_{h} / \eta_{o}$ was ranged between $1.97 \times 10^{-2}$ to $5.13 \times 10^{-2}$ in the study. In the expression of Reynolds number, $U$ and $D_{h}$ are the average fluid velocity and hydraulic diameter of the channel. Using the estimated diffusion coefficient of the magnetic particles in mineral oil, the Peclet number $P e=w U / D_{f}$ was ranged between $7.45 \times 10^{5}$ to $1.94 \times 10^{6}$. In the experiment 
with ferrofluid-cladding configuration [Figure 5-2(c)], mineral oil was delivered into the middle inlet with a flow rate $Q_{o}$ ranging from 0.2 to $0.6 \mathrm{ml} / \mathrm{h}$, while the flow rate of ferrofluid in the two sheath streams $Q_{F F}$ was fixed at $0.05 \mathrm{ml} / \mathrm{h}$. In this case, the corresponding range for the Reynolds number and the Peclet number were the same as in the case of ferrofluid core. The Reynolds number and Peclet number in the experiments ensured that the flow in the experiments was laminar, and diffusive mixing between the streams was negligible [206].

\subsection{Experimental Results}

(a)

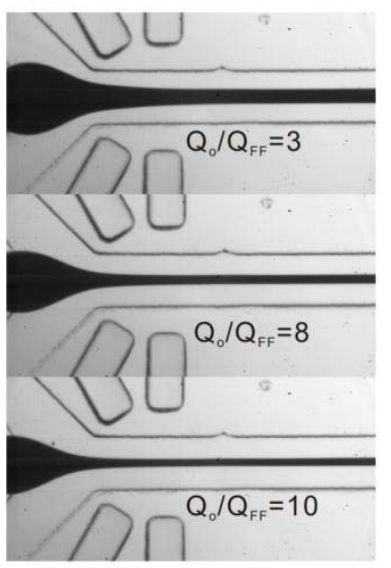

(c) $Q_{0} / Q_{\mathrm{FF}}=10$ (b)
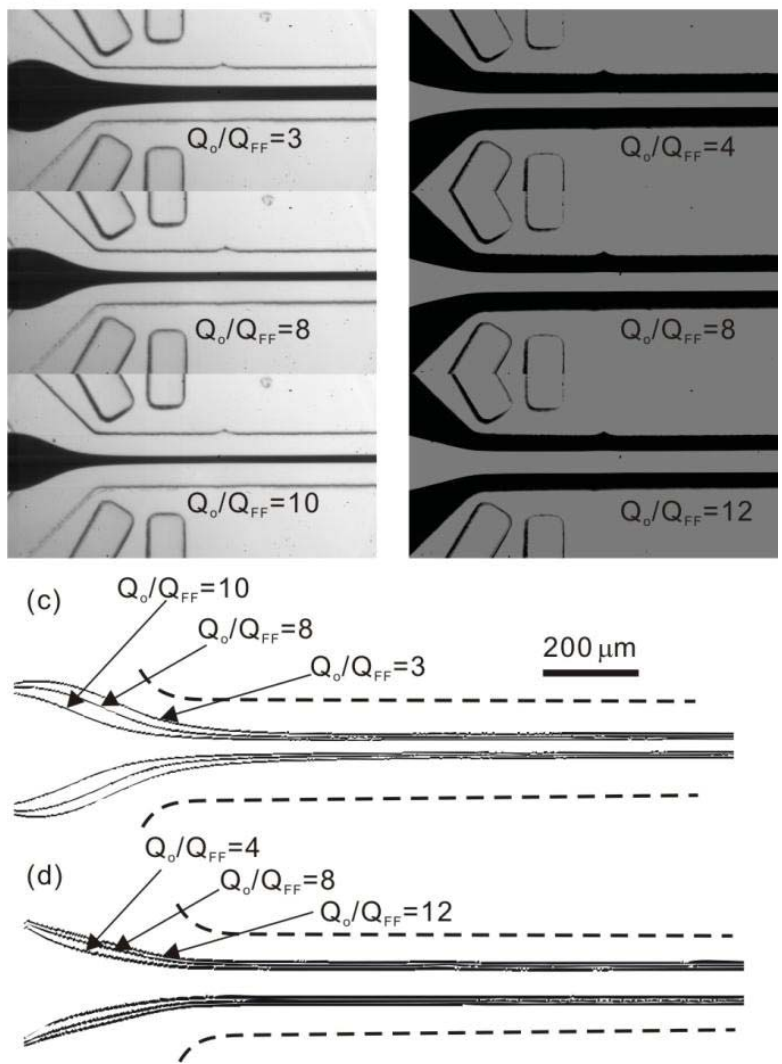

Figure 5-6 Hydrodynamic spreading in a microchannel. The flow rate of ferrofluid is fixed at $0.05 \mathrm{ml} / \mathrm{h}$ : (a) Ferrofluid-core configuration; (b) Ferrofluid-cladding configuration; (c) Overlapped interfaces of hydrodynamic spreading for ferrofluid-core configuration; (d) Overlapped interfaces of hydrodynamic spreading for ferrofluid-cladding configuration. 
In a conventional hydrodynamic spreading, the relative width of the middle stream can be estimated as $w=\left(1+\left(\eta_{\text {sheath }} / \eta_{\text {core }}\right)\left(Q_{\text {sheath }} / Q_{\text {core }}\right)\right)^{-1}$ [207]. Without a magnetic field, the width of the core stream can be adjusted by hydrodynamic means such as the flow rate ratio or viscosity ratio. In this study, the hydrodynamic spreading was achieved by varying the flow rate ratio. The fluid distribution of hydrodynamic spreading in a microchannel is shown in Figure 5-6(a) for ferrofluid-core and Figure 5-6(b) for ferrofluid-cladding configuration. The corresponding interfaces were extracted and presented in Figure 5-6(c) for ferrofluid-core and Figure 5-6(d) for ferrfoluid-cladding configuration. The spreading of the liquid/liquid interface occurs along the entire flow system. Both the channel width and depth would affect the distribution of the flow. For a fixed channel width, the change of channel depth would alter the contact area for mixing. For a fixed channel depth, the change of channel width would alter the concentration gradient which would strongly affect the magnetic force. In this investigation, the effects of channel parameters were not considered.

In the spreading experiment with ferrofluid-core configuration shown in Figure 5-2(a), the core width will increase under an applied uniform magnetic field, because of the pulling force (Eq. (5.8)) and the migration of magnetic particles due to the gradient in susceptibility between the ferrofluid and the mineral oil. In the spreading experiment with ferrofluid-cladding configuration shown in Figure 5-2(b), the core width decreases because of the spreading of the ferrofluid cladding streams. 
(a)

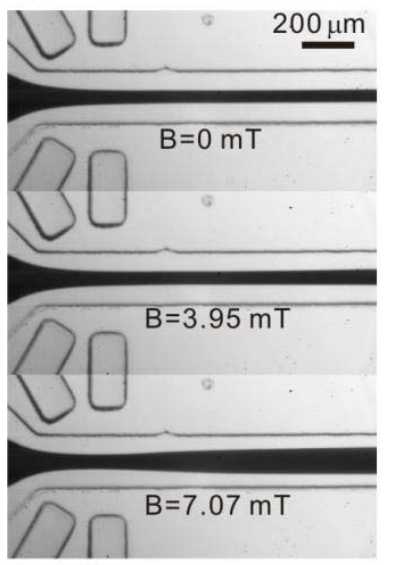

(b)

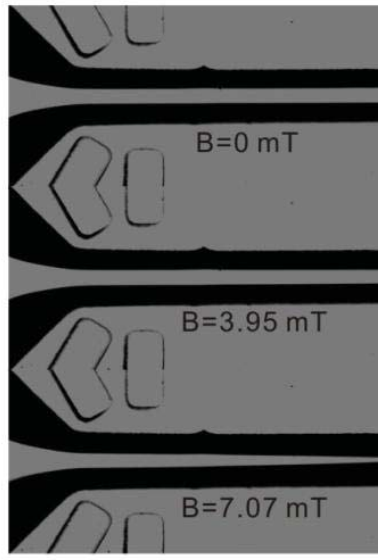

(c)

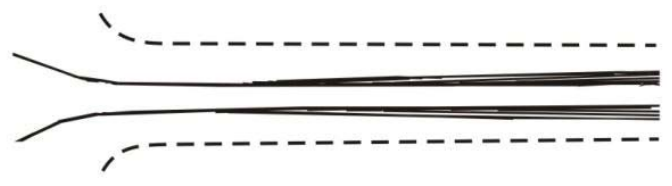

(d)

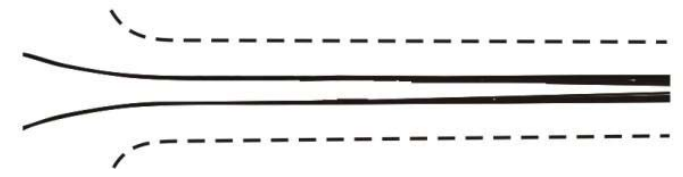

Figure 5-7 Magnetofluidic spreading in a microchannel. The flow rates of ferrofluid and mineral oil are fixed at $0.05 \mathrm{ml} / \mathrm{h}$ and $0.2 \mathrm{ml} / \mathrm{h}$, respectively: (a) Ferrofluid-core configuration; (b) Ferrofluid-cladding configuration; (c) Overlapped interfaces of magnetofluidic spreading with ferrofluid-core configuration; (d) Overlapped interfaces of magnetofluidic spreading with ferrofluid-cladding configuration.

Figure 5-7(a) and (b) show the typical images of the fluid system at different applied magnetic flux densities for the two spreading cases. The results clearly show an entrance effect. Without the applied magnetic field ( $B=0 \mathrm{mT}$ in Figure 5-7(a)), the core stream is focused to a constant width which is established at about two channel widths downstream from the entrance. To investigate the effect of magnetic field on the interface between the ferrofluid and the mineral oil, a customized image processing program written in MATLAB is used to detect and plot the overlapping interfaces at different flux densities for magnetofluidic spreading with both ferrofluid-core (Figure 5-7(c)) and ferrofluid-cladding (Figure 5-7(d)) configuration. 
With a moderate magnetic field, the interface at the entrance remains unchanged as shown in Figure 5-7(d). The width gradually changes along the flow direction to reach a constant value. The entrance is significantly longer than that of purely hydrodynamic spreading (Figure 5-6(d)).
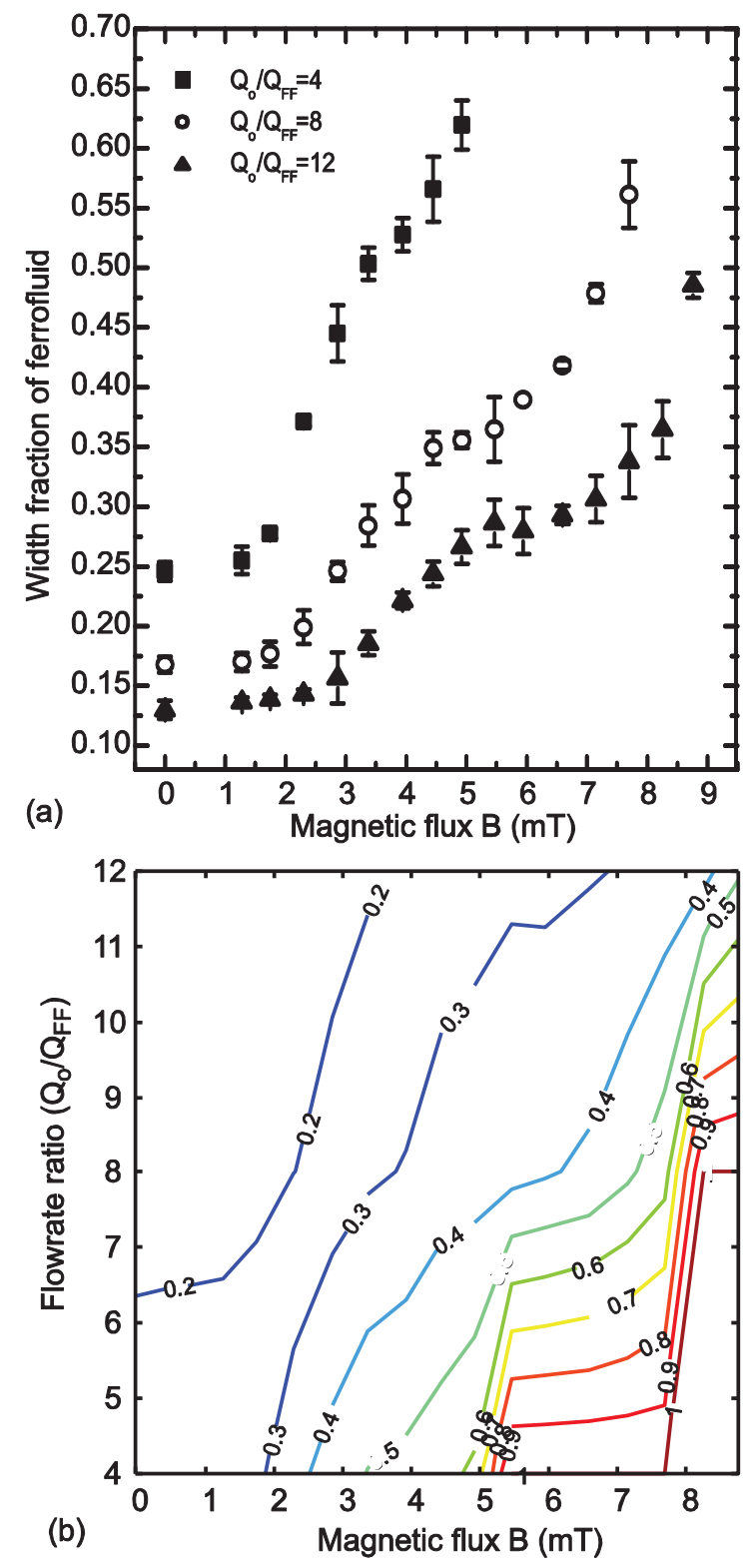

Figure 5-8 Magnetofludic spreading with ferrofluid-core configuration: (a) Normalized width of the core stream as functions of flux density; (b) Contour map of normalized width.

Figure 5-8(a) and (b) show the experimental results for the spreading with ferrofluid-core configuration. The spreading effects are characterized by the width 
fraction of the ferrofluid core stream. As shown in Figure 5-8(a), the spreading effect is more apparent under larger magnetic flux value. Upon reaching a critical value of magnetic flux, instability occurs. As mentioned above, the experiments were carried out with fixed $Q_{\mathrm{FF}}$ and various $Q_{\mathrm{o}}$. The flow rate ratio is defined as the ratio of $Q_{\mathrm{o}}$ to $Q_{\mathrm{FF}}$. For the spreading with ferrofluid-core configuration, a larger magnetic flux is required to achieve spreading at a higher flow rate ratio. To characterize the spreading effect with flow rate ratio and applied magnetic flux, a contour map is provided in Figure 5-8(b) for the normalized width of the core stream. The contour map offers an operating diagram of the magnetofluidic spreading with ferrofluid-core configuration.

Experiments were also carried out to investigate the spreading phenomena with ferrofluid-cladding configuration. Similarly, the spreading effects are illustrated in terms of the width fraction of the mineral oil core stream. However, the width of the core stream decreases under a larger magnetic flux value as the magnetic nanoparticles diffuse toward the diamagnetic core stream. For increasing magnetic flux, the two cladding ferrofluid steams spread until they overlap. Figure 5-9(a) and (b) shows the experimental results for the ferrofluid-cladding case. The flow rate ratio was set to be the same as the ferrofluid-core case. 

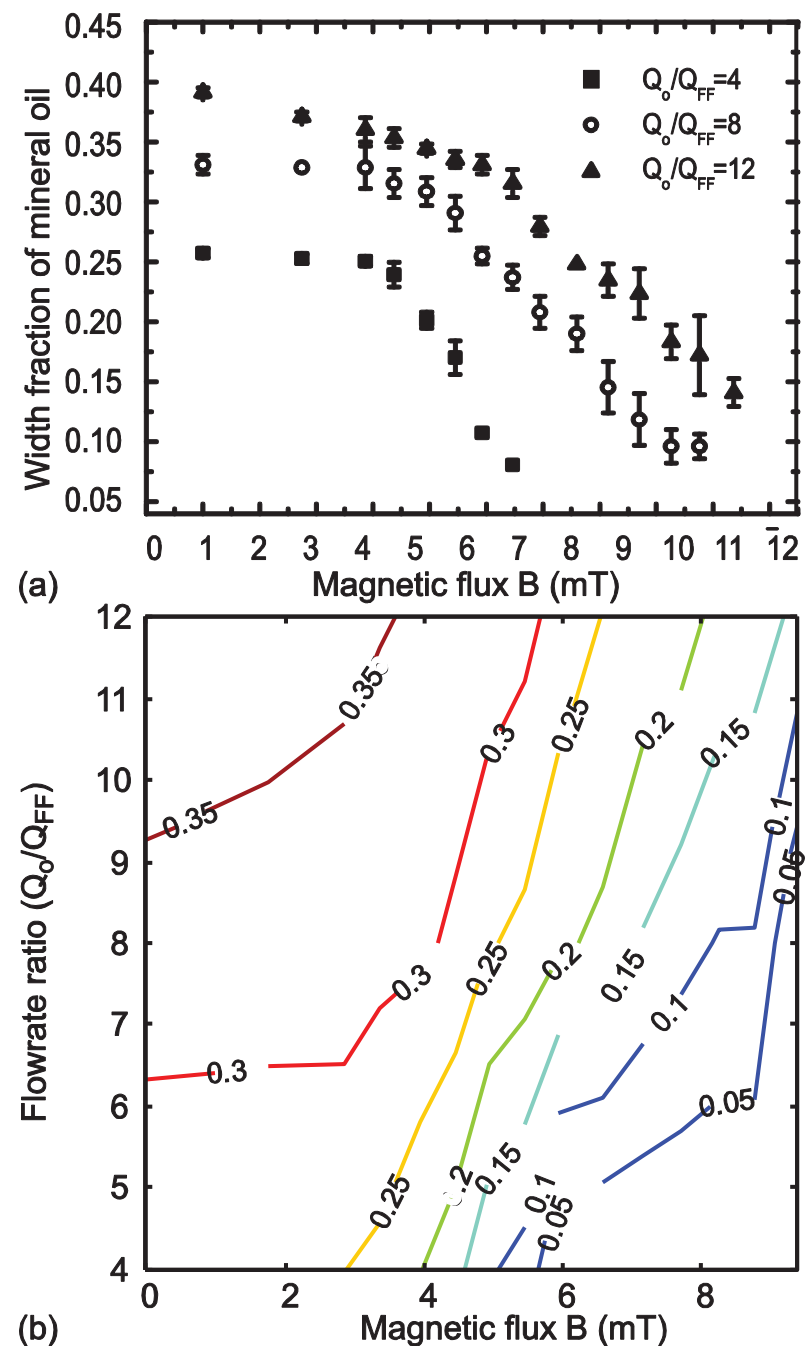

Figure 5-9 Magnetofludic spreading with ferrofluid-cladding configuration: (a) Normalized width of the core stream as functions of flux density; (b) Contour map of normalized width.

\subsection{Numerical Simulations}

In addition to the experimental investigation, numerical simulation was carried out with a 3D model to obtain qualitative insights into the physics of the magnetofluidic spreading phenomena. 


\subsubsection{Numerical Model}

All dimensions of the channel follow those of the fabricated device used in the experiment. At the inlet, the core flow and sheath flows are taken to be fully developed with an average velocity $U$ of,

$$
U=\frac{Q}{w h}
$$

where $Q$ is the volumetric flow rate of each inlet stream. No-slip condition is enforced at the walls. No flux condition is employed at the walls for solving the concentration distribution. Outflow condition is used at the outlet. The continuum and incompressible assumptions are used in the model. The mass, energy and momentum are assumed to conserved for a fixed volume. The electromagnetic induction is not considered as the uniform magnetic field is relatively stable. The coupling of magnetic field and electric field is neglected. Except that the liquids are miscible, all the assumptions for flow field and magnetic field are the same as presented in Section 3.2.1.

For an unsteady and viscous system, the continuity and momentum governing equations are presented as,

$$
\begin{gathered}
\frac{\partial \rho}{\partial t}+\nabla \bullet(\rho \vec{u})=0 \\
\frac{\partial}{\partial t}(\rho \vec{u})+\nabla \bullet(\rho \vec{u} \vec{u})=-\nabla P+\nabla \bullet\left[\mu\left(\nabla \vec{u}+\nabla \vec{u}^{T}\right)\right]+\vec{F}
\end{gathered}
$$

In addition, the governing equation for concentration $C$ is expressed as,

$$
\frac{\partial C}{\partial t}+\left(\vec{u}_{p} \cdot \nabla\right) C=D_{f} \nabla^{2} C
$$


The ferrofluid diffusion related to a mass transfer of magnetic particles is governed by Eq. (5.4) with $C$ representing the non-dimensional volume concentration and $\vec{u}_{p}$ indicating the velocity of the magnetic particles. The diffusion coefficient $D_{f}$ is taken to be constant. The action of the magnetic field results in the rotation of magnetic particles suspended in the ferrofluid. It is reported that the hindrance of the rotation leads to flow resistance and exhibits magnetoviscous effect [208]. As stated previously, the angular term is omitted in the momentum conservation equation for the ferrofluid which is slightly shear dependent. In addition to the hydrodynamic flow field, the velocity of a particle due to magnetic force is given by Stokes drag law. Through balancing the hydrodynamic force and magnetic force on each particle, the particle velocity $\vec{u}_{p}$ can be expressed as,

$$
\vec{u}_{p}=\vec{u}+\vec{u}_{m a g}
$$

where $\vec{u}_{\text {mag }}=\vec{F}_{\text {mag }} / 6 \pi \mu r_{p}$ with $r_{p}$ representing the radius of the magnetic particles. The magnetic force on a single particle $\vec{F}_{\text {mag }}$ can be expressed as,

$$
\vec{F}_{\text {mag }}=\frac{1}{2} V_{p} \mu_{0} \nabla \chi \vec{H}^{2}
$$

where $V_{p}$ is the volume of a single particle, $\chi$ is the susceptibility of the mixed fluid, $\mu_{0}$ is the permeability of free space which has a constant value of $4 \pi \times 10^{-7} N A^{-2}$, and $\vec{H}$ is the strength of the applied magnetic field. In the concentration equation, the diffusion coefficient will be affected by magnetic force according to the reports by Blums [209-210]. Compared with the value at zero field strength, the diffusion coefficient will be increased by two times by a field strength of $200 \mathrm{kA} / \mathrm{m}$. The increase of diffusion coefficient is omitted in this work as the field strengths in our 
experiments are much lower than that reported by Blums. It is worth noting that there is a false numerical diffusion in the simulation even if the second order upwind scheme is employed for coupling advection and diffusion. Taylor dispersion is also not included in the numerical simulation. The effect of Taylor dispersion is not analyzed as Taylor dispersion also existed in the experimental investigation. If Taylor dispersion affects a lot, the diffusion in the experiment will also be strong. The diffusion observed in our experiment is not significant, so we considered the strong diffusion in the numerical simulation to be the false diffusion. As a result, the magnitude of the magnetic force in the simulation is much smaller than the value in the experiment because of the lower concentration gradient caused by numerical diffusion. In the present work, the magnetic force is compensated by a correction factor to fit the simulation results with the experimental data. The correction factor is 1000 for ferrofluid-core and 100 for ferrofluid-cladding case. Thus, the numerical results reported in this chapter only provide a qualitative understanding of the underlying physics of magnetofludic spreading.

The forces acting on the fluid in magnetofluidic spreading $\vec{F}$ consists of gravitational force $\vec{F}_{g}$, interfacial force $\vec{F}_{\sigma}$ and magnetic force $\vec{F}_{m}$. In this numerical simulation, the gravitational force is negligible due to the small scale involved. In addition, interfacial force does not exist as the system consists of two miscible fluids. The magnetic force can be calculated by introducing the magnetic scalar potential $\psi$ in the form of $\vec{H}=-\nabla \psi$. For the numerical implementation with miscible fluids, the susceptibility is a function of the concentration of the magnetic particles $\chi=\chi(C)$ 
[210]. A linear dependence of susceptibility on the concentration is assumed. The expression for magnetic potential equation is,

$$
\nabla \bullet\left[\left(1+\chi_{m}\right) \nabla \psi\right]=0
$$

The corresponding magnetic force in a uniform field $\vec{F}_{m}$ is calculated by,

$$
\vec{F}_{m}=-\frac{1}{2} \mu_{0}|\vec{H}|^{2} C_{V} \nabla \chi
$$

where $C_{V}$ is the volume concentration of the particles which can be represented by $C_{V}=C_{V 0} C$ with the initial value of $C_{V 0}=0.04$. The non-dimensional volume concentration $C$ is determined by Eq. (5.4).

The variation of density $\rho$ and viscosity $\eta$ as a function of concentration $C$ is determined as [162],

$$
\begin{gathered}
\rho=C \rho_{F F}+(1-C) \rho_{o} \\
\eta=\eta_{F F} e^{S(1-C)}
\end{gathered}
$$

where $\rho_{F F}$ and $\rho_{\mathrm{o}}$ are the densities of the magnetic fluid (ferrofluid) and mineral oil respectively. The viscosity parameter $S$ can be expressed as $S=\ln \left(\eta_{o} / \eta_{F F}\right)$ with $\eta_{F F}$ and $\eta_{\mathrm{o}}$ representing the viscosities of ferrofluid and mineral oil, respectively.

The governing equations listed above are solved by using FVM on a Cartesian staggered grid. The SIMPLER algorithm is employed for coupling between the pressure and the velocity fields to improve convergent efficiency of the calculation [211]. The second order upwind scheme with superbee limiter function is used for formulating the discretization equation involving convection and diffusion. The time integration is achieved by using the fully implicit scheme. 


\subsubsection{Numerical Results}
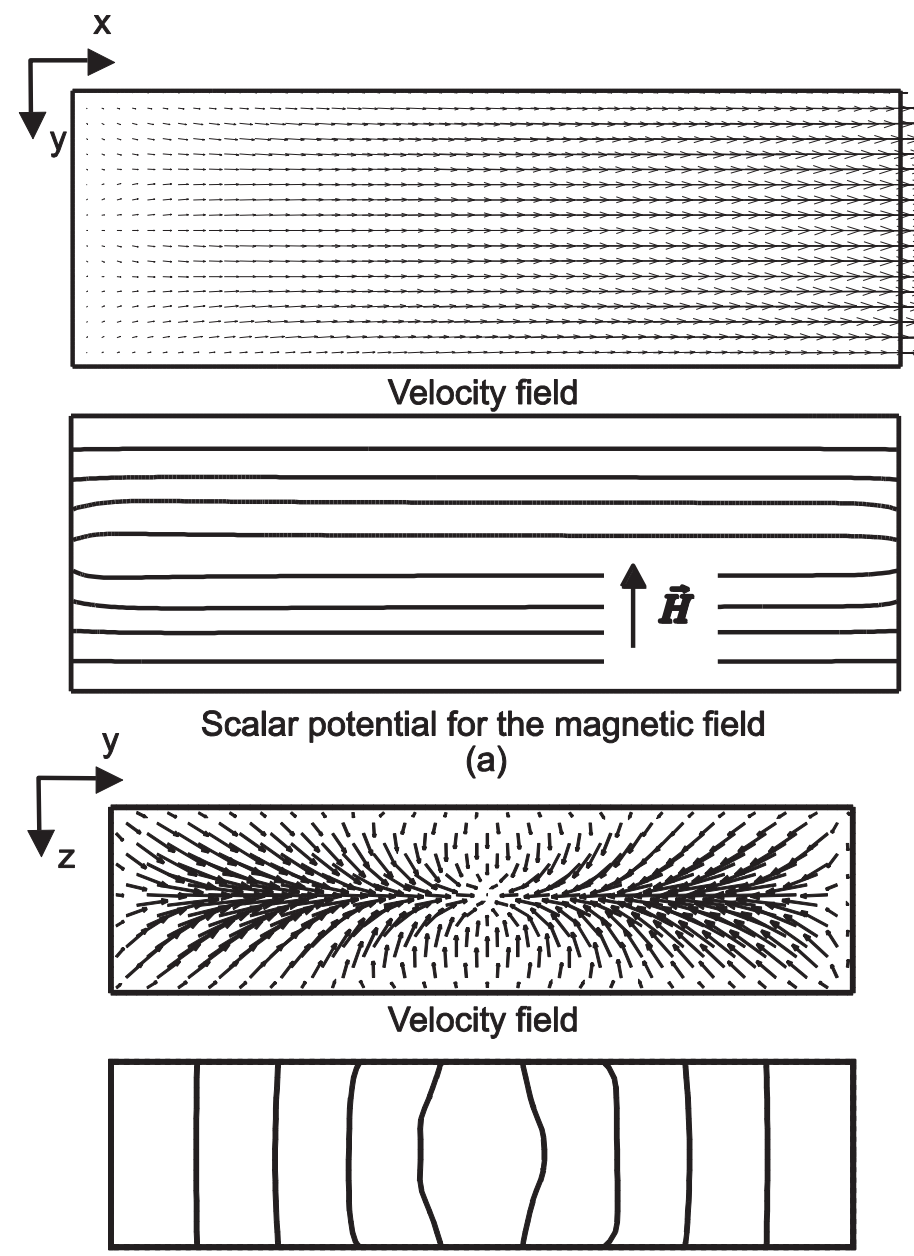

Scalar potential for the magnetic field

(b)

Figure 5-10 Spreading with ferrofluid-core configuration at $\mathrm{B}=2.87 \mathrm{mT}, Q_{d} / Q_{F F}=4$ : (a) $\mathrm{x}-\mathrm{y}$ plane; (b) y-z plane. 


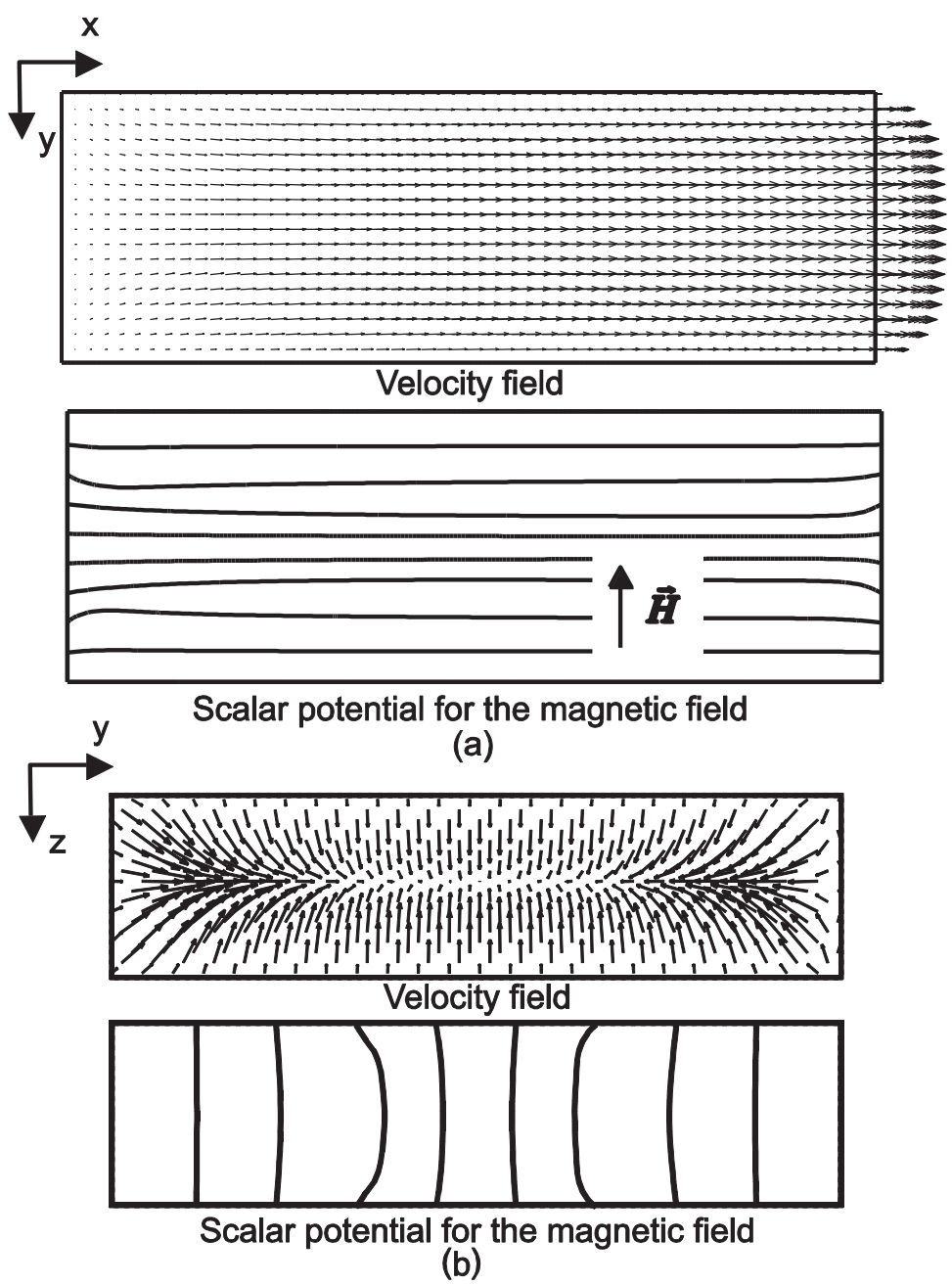

Figure 5-11 Spreading with ferrofluid-cladding configuration at B=2.87 mT, $Q_{o} / Q_{F F}=4$ : (a) x-y plane; (b) y-z plane.

The numerical study focuses on the spreading phenomenon under an external magnetic field. The grid independent study was first performed to find a compromise between grid size and accuracy. Calculation error could be reduced with further grid refinement as the numerical diffusion was significantly affected by the grid size. A grid size of $218 \times 74 \times 20$ was finally selected in the simulation, because numerical diffusion could not be considerably reduced with further grid refinement. We first investigate the concentration distribution inside the channel without the magnetic field. Neglecting the gravitational force and interfacial tension force, the flow is thoroughly determined 
by the flow rate and viscosity ratio between the two streams when the field strength is zero. The calculation is implemented with the flow rate ratio fixed at $Q_{o} / Q_{F F}=4$. The typical simulation results of the velocity field and the corresponding scalar potential $\psi$ of the magnetic field under a flux density of $2.87 \mathrm{mT}$ are shown in Figure 5-10 and Figure 5-11 for the ferrofluid-core case and the ferrofluid-cladding case, respectively. Part (a) of the figures shows the results of the middle plane, while part (b) depicts the results of the cross section at the end of the channel. The results show that under a relatively weak magnetic field, the flow is laminar and stable. The velocity flow field of the cross section clearly shows the existence of a secondary flow field that promotes advective mixing at the interface between the ferrofluid and the mineral oil. 

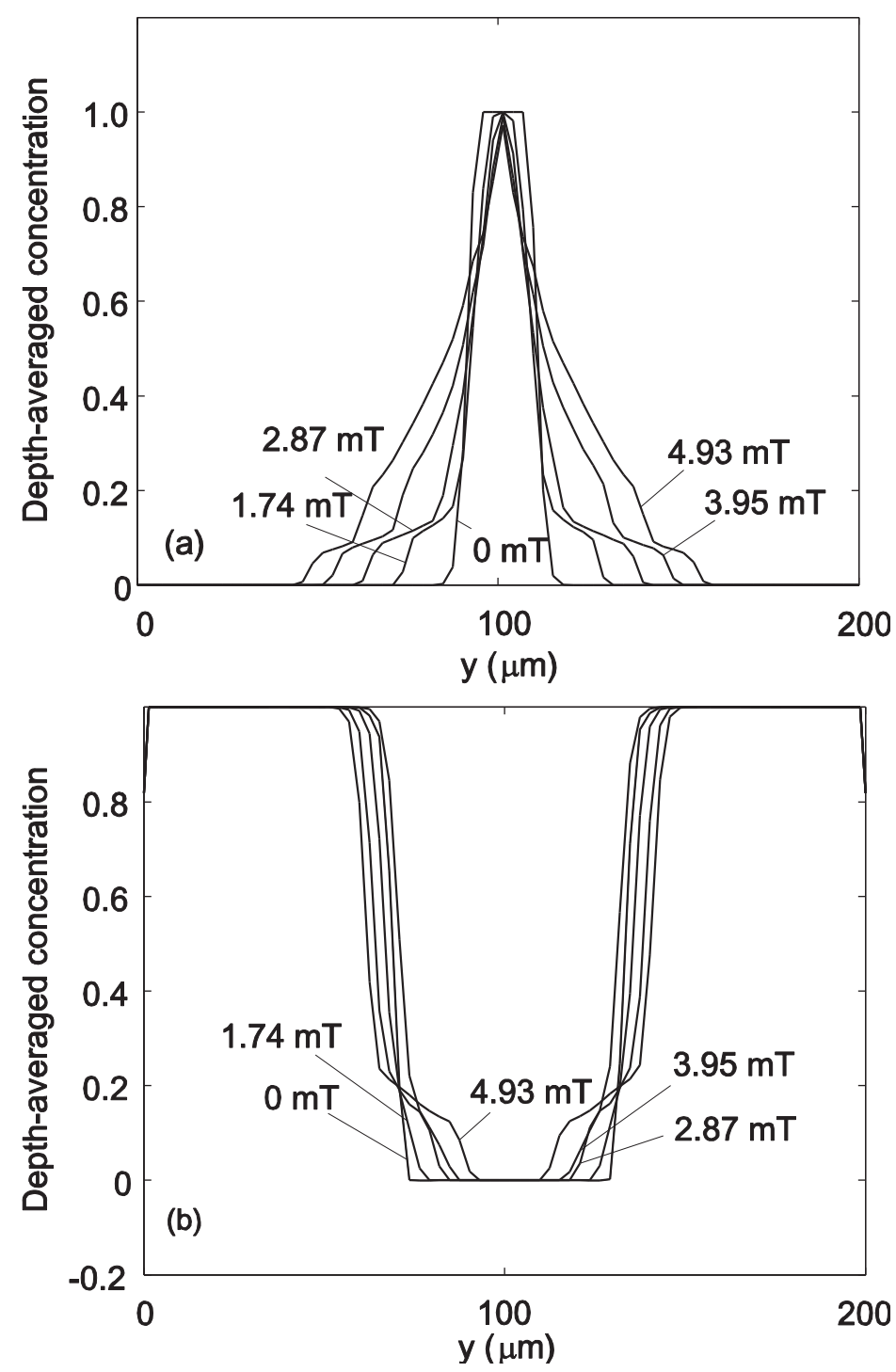

Figure 5-12 Depth averaged concentration across the channel width at different field strengths $\left(Q_{d} / Q_{F F}=4\right)$ : (a) Magnetofluidic spreading with ferrofluid-core configuration; (b) Magnetofluidic spreading with ferrofluid-cladding configuration.

The simulation was carried out with an external magnetic field ranging from 1.74 $\mathrm{mT}$ to $4.93 \mathrm{mT}$. The typical results of the depth averaged concentration of magnetic particles are presented in Figure 5-12(a) and (b), respectively. Due to the secondary flow, the concentration distribution of the magnetic particles is not uniform along the channel depth. Since ferrofluid appears in the recorded images as a dark area with 
almost no grey scales, the simulated concentration needs to be averaged along the channel depth to achieve a realistic comparison with the experimental data.

The spreading effect can clearly be observed in the ferrofluid-core case shown in Figure 5-12(a). The additional drift of particles caused by magnetic force leads to an increase in apparent diffusion coefficient. At a higher flux density, the bulk magnetic force caused by the susceptibility gradient is large enough to induce instability or secondary flow at the interface of the ferrofluid and the oil. The concentration profile shows a typical broad base caused by the secondary flow as shown in Figure 5-12(a). This secondary flow will be dominant at a higher flux density leading to complete mixing in the microchannel. As shown in Figure 5-12(b), a similar spreading behaviour can be observed in the ferrofluid-cladding case. A stronger broadening at the interface at high flux density can also be observed. However, due to the dominant hydrodynamic transport at the center of the channel, the effect of the secondary flow in the ferrofluid-cladding case is not as strong as in the ferrofluid-core case. 

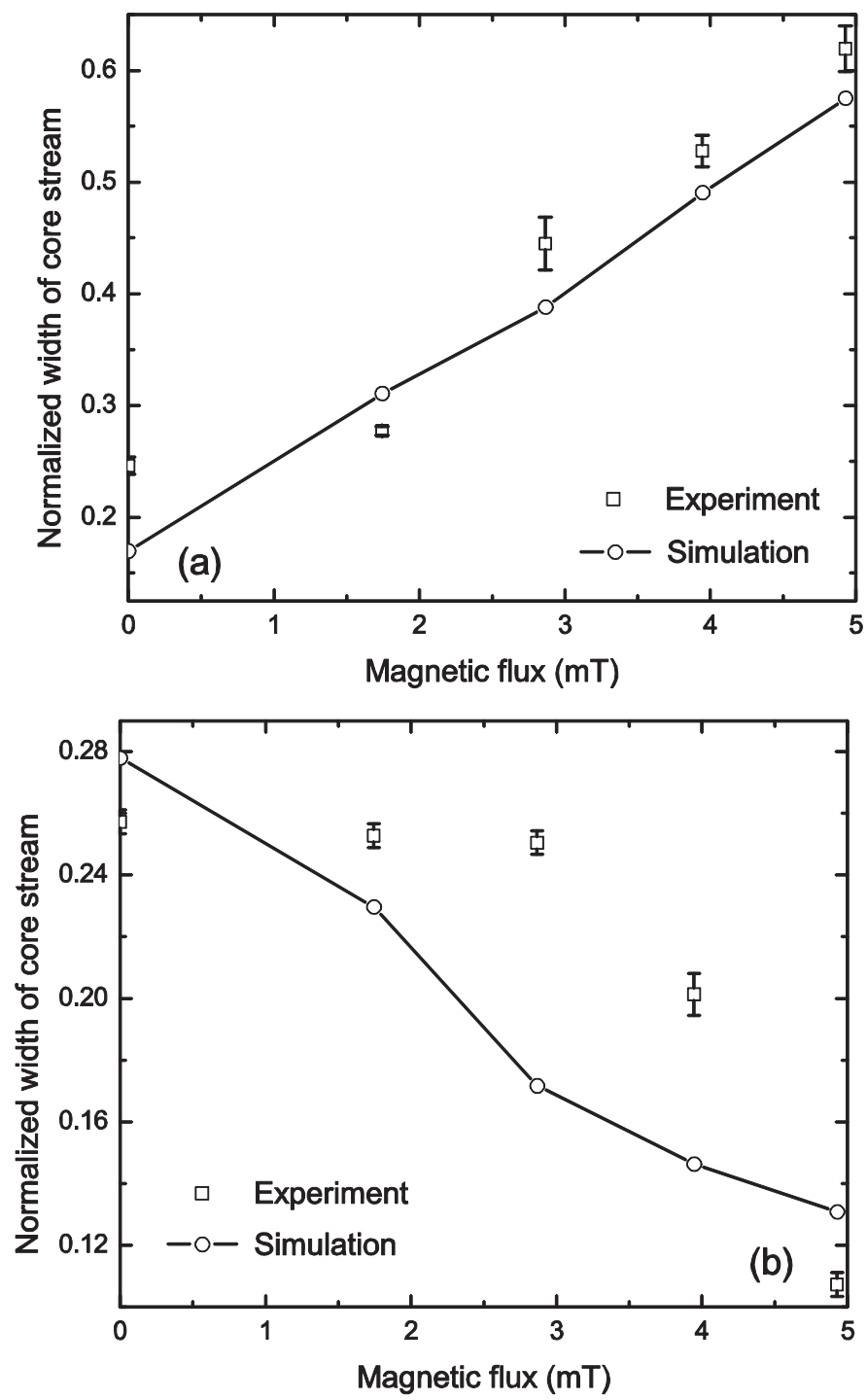

Figure 5-13 Comparison between experimental and numerical results with $Q_{\delta} / Q_{F F}=4$ : (a) Magnetofluidic spreading with ferrofluid-core configuration; (b) Magnetofluidic spreading with ferrofluid-cladding configuration.

Experimental and numerical results are compared based on the normalized width of the core stream at the corresponding field strength. Based on the depth-averaged concentration, the width of the core stream can be determined from the simulation. In the computational domain, the width of the core stream is evaluated at the end of the channel. Figure 5-13 compares the simulation results with the experiment results. Without an external magnetic field the core flow is focused to a constant value as 
observed in the experiments. In the presence of the magnetic field, the drift of magnetic particles leading to improved apparent diffusion and mixing at the interface lead to the expansion of the ferrofluid core. The core width increases with increasing field strength. The same phenomenon is observed in both experiment and numerical simulation. The normalized width of the core stream in both experiment and numerical simulation are compared for the ferrofluid-core case and the ferrofluid-cladding case in Figure 5-13(a) and (b), respectively. Both experimental and numerical results show a similar trend of the relationship between the normalized width and the magnetic flux density. The simulation gives good insights into the underlying physics of the observed spreading phenomena. However, more effort is required for a quantitative description of magnetofluidic spreading, especially overcoming the problem of numerical diffusion.

\subsection{Summary}

This study investigates experimentally and numerically the phenomenon of magnetofluidic spreading of ferrofluid as core and cladding in a three-stream flow system. The magnetofluidic spreading effect is investigated at the presence of an external uniform magnetic field. In the case of ferrofluid cladding, the width of the core stream is evaluated as a function of the applied magnetic flux density. As the field strength is increased, a stronger spreading effect can be observed. Due to the secondary flow, the ferrofluid-core configuration is more sensitive to the applied magnetic field. The numerical simulation shows that magnetofluidic spreading is 
caused by increased apparent diffusion due to drift of magnetic particles and secondary flow at the ferrofluid/oil interface. Both phenomena are caused by a susceptibility gradient or the concentration gradient of magnetic particles in the ferrofluid. Both experiment and simulation show a similar behaviour of the flow system. However, current numerical model can only provide a qualitative investigation of the phenomenon. Computational schemes to overcome numerical diffusion need to be considered to have qualitative results, because the interaction between magnetic field and the flow field is extremely sensitive to the gradient of particle concentration. 


\section{CHAPTER 6}

\section{RAPID MAGNETOFLUIDIC MIXING IN A UNIFORM MAGNETIC FIELD}

Magnetofluidic micromixers reported previously in the literature rely on the magnetic force caused by a field gradient. This field gradient is often generated by an integrated electromagnet, which in turn induces a significant amount of heat and requires a complex fabrication process. Furthermore, time-dependent filed gradients require synchronized external electromagnets or a moving permanent magnet leading to a complex overall mixer design. This chapter presents both experimental and numerical investigations of magnetically induced mixing based on the susceptibility gradient between a diamagnetic fluid and a ferrofluid. The micromixer with a circular mixing chamber is designed and fabricated. Instead of using a high magnetic field gradient and a high field strength, the concept described here only needs a uniform magnetic field with a field strength of few milliteslas, two or three orders of magnitude lower than previously required by other magnetic mixing concepts. This concept provides rapid mixing as the magnetic fluid responses instantly with the magnetic field. This concept achieves efficient mixing by enhancing the migration of suspended magnetic nanoparticles and inducing the instability at the liquid-liquid interface. The mixing efficiency is evaluated for different flow rate ratios, which determine the mixing ratio between the ferrofluid and the diamagnetic liquid. Furthermore, the relationship between mixing efficiency and viscosity ratio is also investigated. To adjust the 
viscosity of the diamagnetic fluid, water and glycerol are mixed in a corresponding ratio. In all experiments, the mixing efficiency is evaluated with varying magnetic flux densities. A numerical model based on Finite Volume Method (FVM) is developed to verify the mixing behaviour observed in the experiment.

\subsection{Experimental Setup and Device}

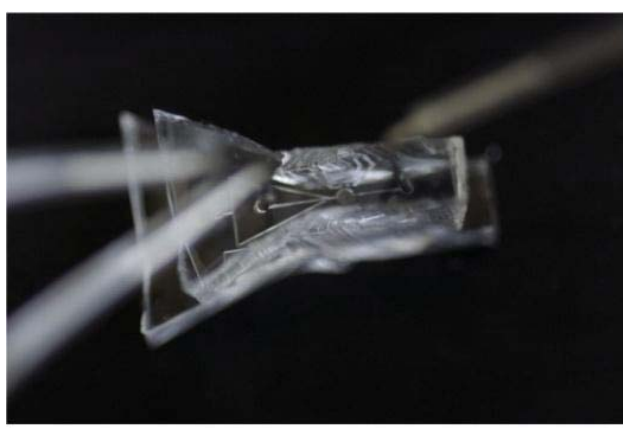

(a)

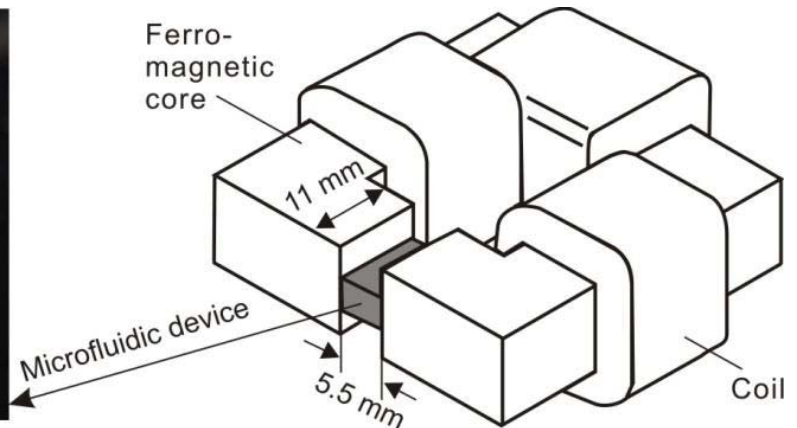

(b)

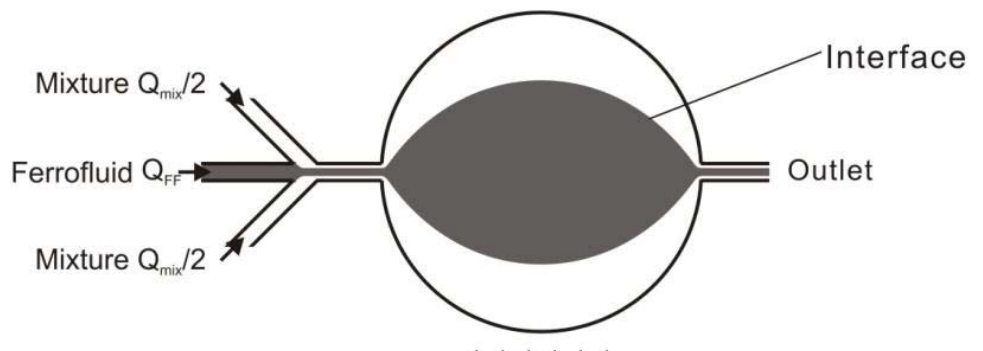

(c)

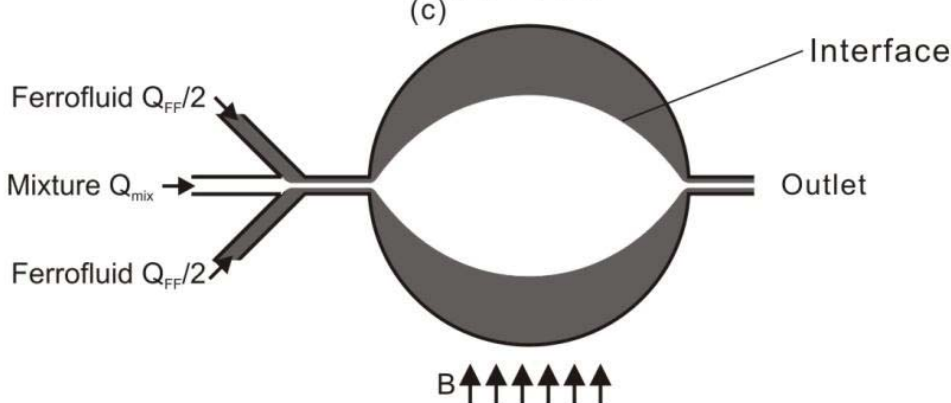

(d)

Figure 6-1 Magnetofluidic mixing in a uniform magnetic field: (a) Fabricated microfluidic device; (b) Custom-made electromagnet; (c) Ferrofluid-core configuration; (d) Ferrofluidcladding configuration. 
The same experimental setup is used as shown in Figure 5-1. The difference is the channel configuration which is shown in Figure 6-1. The picture of the fabricated microfluidic device is given in Figure 6-1(a). The electromagnet was modified from a transformer [Figure 6-1(b)], whose ferromagnetic core was cut to form an air gap of $5.5 \mathrm{~mm}$. The uniform magnetic field in the air gap will apply across the entire microfluidic device. Demagnetization of the electromagnet is necessary to eliminate the remaining magnetization of the core after each round of the experiment. It is achieved by applying a reversed current for around 5-10 minutes with the highest current used in the previous experiment.

A circular chamber was employed for the mixing process between the ferrofluid and a diamagnetic mixture of water and glycerol. The device had three inlets and one outlet. The circular chamber, which is shown in Figure 6-1(c) and (d), had a depth of $h=50 \mu \mathrm{m}$ and a diameter of $d=1 \mathrm{~mm}$. The liquids were introduced into the inlets with a depth of $h=50 \mu \mathrm{m}$ and a width of $w=50 \mu \mathrm{m}$. SU-8 with 2050 resists was spin coated on a cleaned 4-inch silicon wafer. Followed by soft baking process, the SU-8 layer is hardened on the Si substrate (Figure 6-2(a)). The configuration of channels was designed and printed on a $5 \times 5$ inch plastic film. The mask was then used to carry out the UV exposure (Figure 6-2(b)). After the post-exposure bake, isopropanol was used for developing to form the SU-8 mold (Figure 6-2(c)). Figure 6-2(g) was the master mould of our chip. The device was made of polydimethylsiloxane (PDMS) using standard soft lithography technique (Figure 6-2(d)). The PDMS was peeled off and punched for access holes with a $0.75 \mathrm{~mm}$ 
diameter puncher (Figure 6-2(e)). The substrate was then treated with oxygen plasma and bonded to another flat PDMS base (Figure 6-2(f)). The PDMS device was further cut to fit into the air gap of the electromagnet. The microfluidic device was slotted into the air gap for subsequent mixing experiments. A uniform magnetic field could be assumed as the mixing chamber was much smaller than the $5.5 \mathrm{~mm} \times 11 \mathrm{~mm}$ test section of the electromagnet. A magnetic flux density up to $50 \mathrm{mT}$ could be generated by tuning the current up to $1.8 \mathrm{~A}$. The magnetic flux generated at different currents was calibrated with a commercial gaussmeter (Hirst, GM05, UK).

\section{(a)}

(b)

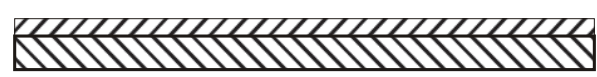

b)

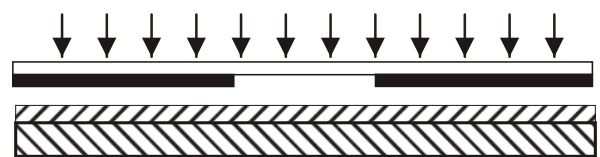

(c)

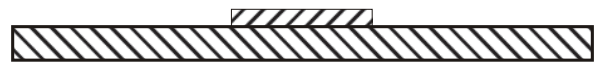

(d)

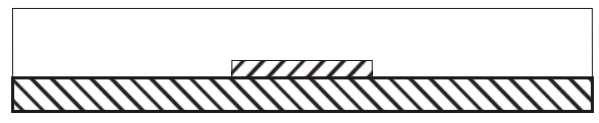

(e)

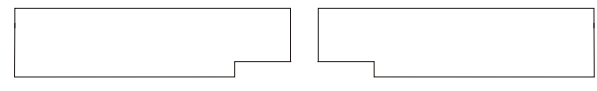

(f)

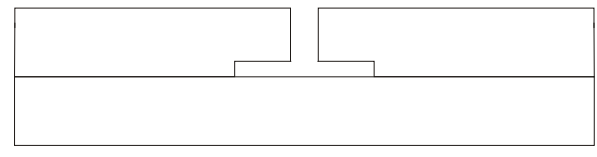

(g)

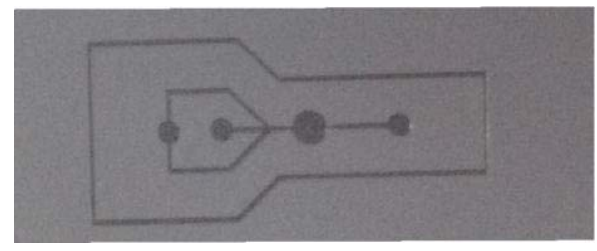

$\mathrm{Si}$ SU-8 PDMS

Figure 6-2 Fabrication of microchannels with soft lithography: (a) SU-8 spin coated on a silicon wafer; (b) UV exposure with a mask; (c) development of SU-8 mold; (d) pouring PDMS on the mold; (e) peeling off the PDMS; (f) surface treatment and bond; (g) the fabricated microchannel. 
Water-based ferrofluid (EMG707, Ferrotec) was used with super paramagnetic nanoparticles well dispersed in the carrier fluid. The ferrofluid had a saturation magnetization of $11 \mathrm{mT}$, a density of $\rho_{F F}=1.1 \times 10^{3} \mathrm{~kg} / \mathrm{m}^{3}$, a viscosity at $27^{\circ}$ of $\eta_{F F}=5 \mathrm{mPa} \cdot \mathrm{s}$ and a magnetic particle concentration of $2 \% \mathrm{vol}$ and an initial susceptibility of $\chi_{0}=0.36$. The magnetization of this kind of ferrofluid has been described in Chapter 3. The diamagnetic liquid was a mixture of DI water and glycerol (G7757 Sigma-Aldrich). Glycerol has a density of $\rho_{G}=1.26 \times 10^{3} \mathrm{~kg} / \mathrm{m}^{3}$, and a viscosity at $20^{\circ}$ of $\eta_{G}=1410 \mathrm{mPa} \cdot \mathrm{s}$. In order to characterize the effect of viscosity mismatch, different water/glycerol mixtures were used. The viscosity and density of the mixture is listed in Table 6-1 for different water/glycerol composition. In the absence of the magnetic field, a clear interface is formed between the ferrofluid and the diamagnetic fluid, as shown in Figure 6-1(c) and (d). At a temperature of $\mathrm{T}=300 \mathrm{~K}$, the diffusion coefficients of the magnetic nanoparticles $\left(d_{\mathrm{p}}=10 \mathrm{~nm}\right)$ into the different water/glycerol mixtures are estimated by Einstein's model, $D_{f}=k_{B} T / 3 \pi \eta_{m i x} d_{p}=1.76 \times 10^{-11} \mathrm{~m}^{2} / \mathrm{s}$ $8.79 \times 10^{-12} \mathrm{~m}^{2} / \mathrm{s}, \quad 5.86 \times 10^{-12} \mathrm{~m}^{2} / \mathrm{s}$, respectively.

Table 6-1 Different water/glycerol mixture.

\begin{tabular}{ccc}
\hline Composition & Viscosity $\eta_{\text {mix }}(\mathrm{mPa} \cdot \mathrm{s})$ & Density $\rho_{\text {mix }}\left(\mathrm{kg} / \mathrm{m}^{3}\right)$ \\
\hline $65.6 \mathrm{wt} \%$ DI water and 34.4 wt \% glycerol & 2.5 & $1.09 \times 10^{3}$ \\
$50 \mathrm{wt} \%$ DI water and 50 wt\% glycerol & 5 & $1.13 \times 10^{3}$ \\
$42.7 \mathrm{wt} \%$ DI water and 57.3 wt\% glycerol & 7.5 & $1.15 \times 10^{3}$
\end{tabular}


The experiments were carried out with ferrofluid-core and ferrofluid-cladding configuration, as shown in Figure 6-1(c) and (d). In the ferrofluid-core configuration, the ferrofluid acted as the core stream while the diamagnetic liquid worked as the cladding stream. In the ferrofluid-cladding configuration, the ferrofluid served as the cladding stream while the diamagnetic liquid worked as the core stream. The distribution of magnetic particles determines the magnetic force. The magnetic force induces a secondary flow which will affect the particle distribution in return. In the case of the ferrofluid-core configuration, the ferrofluid was delivered into the middle inlet at a constant flow rate of $0.5 \mathrm{ml} / \mathrm{h}$. The water/glycerol mixture served as cladding streams with a flow rate varying from 0.25 to $0.75 \mathrm{ml} / \mathrm{h}$. Based on the properties of the water/glycerol mixture, the Reynolds number $\operatorname{Re}=\rho_{\text {mix }} U D_{\mathrm{h}} / \eta_{\text {mix }}$ were $1.73 \times 10^{-1}$ $2.88 \times 10^{-1}, 0.896 \times 10^{-1}-1.49 \times 10^{-1}$ and $6.07 \times 10^{-2}-1.01 \times 10^{-1}$, respectively. Using the estimated diffusion coefficient of the magnetic particles in water/glycerol mixture, the Peclet number $P e=w U / D_{f}$ were $2.37 \times 10^{5}-3.95 \times 10^{5}, 4.74 \times 10^{5}-7.90 \times 10^{5}$ and $7.11 \times 10^{5}-1.18 \times 10^{6}$, respectively.

In order to further investigate the effect of flow rate on the mixing behaviour, experiments were also carried out with flow rates of core and cladding stream varying from 0.25 to $0.75 \mathrm{ml} / \mathrm{h}$ but maintaining a fixed flow rate ratio of 1 . The corresponding Reynolds number were $1.15 \times 10^{-1}-3.45 \times 10^{-1}, 0.597 \times 10^{-1}-1.79 \times 10^{-1}$ and $4.05 \times 10^{-2}$ $1.21 \times 10^{-1}$. The Peclet number were $1.58 \times 10^{5}-4.74 \times 10^{5}, 3.16 \times 10^{5}-9.48 \times 10^{5}$ and $4.74 \times 10^{5}-1.42 \times 10^{6}$ 
In the case of ferrofluid-cladding configuration, the ferrofluid was delivered into the sheath inlets at a constant flow rate of $0.5 \mathrm{ml} / \mathrm{h}$. The water/glycerol mixture served as the core stream with flow rate varying from 0.25 to $0.75 \mathrm{ml} / \mathrm{h}$. The corresponding Reynolds number and Peclet number were the same as in the case of ferrofluid-core experiment. The Reynolds number and Peclet number ensured a laminar flow in the channel and negligible diffusive mixing.

Evaluating the quality of mixing inside the mixing chamber is normally difficult because of the circular shape of the chamber, and the nonlinear correlation between grey scale of the ferrofluid image and its concentration. An interrogation window was selected in the study inside the chamber covering all three streams for mixing evaluation. The homogeneity of the recorded grey scale was used as a measure for the mixing efficiency. First, the mean value of the grey scale $\bar{I}$ of the window is determined. Next the standard deviation of the grey scale values in the interrogation window is evaluated,

$$
S_{N}=\sqrt{\frac{1}{N} \sum_{i=1}^{N}\left(I_{i}-\bar{I}\right)^{2}}
$$

If the window is homogeneous, the value of the standard deviation will be $s_{N}=0$. The mixing efficiency is subsequently determined as $\vartheta=1-s_{N}$.

\subsection{Numerical simulation}

Because of the shallow circular chamber, the numerical simulation was realized with a 2D model to gain more insights into the physics of the mixing process. The dimensions of the circular chamber used in the simulation are the same as those of the 
experiments. A fully developed velocity profile is assumed for the inlets of both the core flow and the sheath flow,

$$
u=-\frac{3}{2} \frac{Q}{w h}\left(\frac{y^{2}-(h / 2)^{2}}{(h / 2)^{2}}\right)
$$

where $Q$ is the volumetric flow rate of the flows, w and h are the width and height of the channel, $-\mathrm{h} / 2 \leq \mathrm{y} \leq \mathrm{h} / 2$ is the coordinate along the channel height. No-slip condition is employed at the walls. No flux condition is employed at the walls for solving the concentration distribution. The exit is set at outflow condition. The continuum and incompressible assumptions are used in the model. The mass, energy and momentum are assumed to conserved for a fixed volume. The electromagnetic induction is not considered as the uniform magnetic field is relatively stable. The coupling of magnetic field and electric field is neglected. Except that the liquids are miscible, all the assumptions for flow field and magnetic field are the same as presented in Section 3.2.1.

In Cartesian notation, the continuity and momentum equations are expressed as,

$$
\begin{gathered}
\frac{\partial \rho}{\partial t}+\nabla \bullet(\rho \vec{u})=0 \\
\frac{\partial}{\partial t}(\rho \vec{u})+\nabla \bullet(\rho \vec{u} \vec{u})=-\nabla P+\nabla \bullet\left[\mu\left(\nabla \vec{u}+\nabla \vec{u}^{T}\right)\right]+\vec{F}
\end{gathered}
$$

The concentration distribution in the whole computational domain is determined by the mass transfer of magnetic particles. The non-dimensional advection-diffusion equation for the concentration distribution is given as,

$$
\frac{\partial C}{\partial t}+\left(\vec{u}_{p} \cdot \nabla\right) C=D_{f} \nabla^{2} C
$$


The magnetic force would induce an additional migration velocity to the magnetic particles and affect their diffusive behaviour inside the circular chamber. By balancing the hydrodynamic force and magnetic force on each particle, the velocity $\vec{u}_{p}$ is given by Stokes drag,

$$
\vec{u}_{p}=\vec{u}+\vec{u}_{m a g}=\vec{u}+\vec{F}_{m a g} / 6 \pi \eta r_{p}
$$

where $r_{p}$ is the radius of the magnetic nanoparticles, $\vec{F}_{\text {mag }}$ is the magnetic force on each particle which can be expressed as,

$$
\vec{F}_{\text {mag }}=\frac{1}{2} V_{p} \mu_{0} \nabla \chi \vec{H}^{2}
$$

where $V_{p}$ is the volume of a single particle, $\chi$ is the susceptibility of the mixed fluid, $\mu_{0}$ is the permeability of free space which has a constant value of $4 \pi \times 10^{-7} N A^{-2}$, and $\vec{H}$ is the strength of the applied magnetic field. By using the concept of volume fraction [162], the density and viscosity can be determined by,

$$
\begin{gathered}
\rho=C \rho_{F F}+(1-C) \rho_{\text {mix }} \\
\eta=\eta_{F F} e^{S(1-C)}
\end{gathered}
$$

where $\rho_{F F}$ and $\rho_{\text {mix }}$ are the densities of the magnetic fluid (ferrofluid) and mixture respectively. The viscosity parameter $S$ can be expressed as $S=\ln \left(\eta_{\text {mix }} / \eta_{F F}\right)$ with $\eta_{F F}$ and $\eta_{m i x}$ representing the viscosities of ferrofluid and mixture, respectively. In the concentration equation Eq. (6.5), the diffusion coefficient $D_{f}$ is taken to be constant as the field strength used in the experiment is relatively low.

In the case of a continuous ferrofluid flow in a circular chamber with an applied magnetic field perpendicular to the stream, the term $\vec{F}$ in the momentum equation consists of gravitational force $\vec{F}_{g}$, interfacial tension $\vec{F}_{\sigma}$ and magnetic force $\vec{F}_{m}$. The 
gravitational force is negligible as the experiment is carried out in a microscale. The interfacial tension can also be omitted as the fluid system is miscible. The magnetic force is calculated by solving the magnetic scalar potential which is introduced in the form of $\vec{H}=-\nabla \psi$. Starting with Maxwell equations, Liu et al. [212] reported the governing equation for magnetic scalar potential and the corresponding magnetic force for an immiscible two-phase flow. In the present work with miscible flow, the susceptibility is a function of particle concentration $\chi=\chi(C)$ [210]. In the calculation, the susceptibility is assumed to vary linearly with the concentration. According to the review by Pankhurst et al. [52] the physical principles behind the bulk magnetic force is the response of magnetic particles to the applied magnetic field. In a uniform magnetic field, the magnetic potential equation and the corresponding magnetic force can be expressed as,

$$
\begin{array}{r}
\nabla \bullet\left[\left(1+\chi_{m}\right) \nabla \psi\right]=0 \\
\vec{F}_{m}=-\frac{1}{2} \mu_{0}|\vec{H}|^{2} C_{V} \nabla \chi
\end{array}
$$

where $C_{V}$ is the volume concentration of the particles which can be represented by $C_{V}=C_{V 0} C$ with the initial value of $C_{V 0}=0.02$.

Without the applied magnetic field, diffusive mixing is supposed to be negligible because of the high Peclet number. However, the diffusion coefficient in the simulation is affected by the so-called false numerical diffusion coefficient. Even with the 2nd order upwind scheme, numerical diffusion still exists in the simulation. As a result, the magnetic force which is proportional to the gradient of the susceptibility is much lower in the simulation than the actual experimental value of a much larger 
gradient. Because of the difficulty to get rid of numerical diffusion, the magnetic force in our simulation is adjusted accordingly to achieve a magnitude comparable to that of the experiment. In ferrofluid-core configuration, the magnetic force is corrected by a coefficient with the value of around 100. However, the numerical diffusion of magnetic liquid is stronger in ferrofluid-cladding configuration. Consequently, a coefficient of 1000 is used for the compensation of numerical diffusion.

The FVM is used to solve the governing equations in a Cartesian staggered grid. The pressure/velocity coupling is achieved with SIMPLER algorithm which is helpful in improving convergent efficiency of the calculation [211]. The 2nd order upwind scheme is employed for discretizating the advection-diffusion equation. The higher the order of the upwind method, the less is the false numerical diffusion. The time integration is proceeded with the fully implicit scheme.

\subsection{Results and discussion}

The results of mixing with ferrofluid-core configuration for different flow rate ratios and flow rates are shown in Figure 6-3. The mixing efficiency is determined from a rectangular interrogation window in the mixing chamber. The discrete experimental points are fitted with the Boltzmann function. Boltzmann function can be expressed as $y=\left(A_{1}-A_{2}\right) /\left(1+e^{\left(x-x_{0}\right) / d x}\right)+A_{2}$, with $A_{1}, A_{2}$ and $x_{0}$ stands for the initial value, the final value and the center. Without a magnetic field, a regular lens shape is formed in the circular chamber with negligible diffusion. In the early stage of increasing magnetic flux, the magnetic force causes the ferrofluid core to spread due to the 
migration of magnetic particles into the diamagnetic fluid. In addition to the hydrodynamic velocity, the magnetic particles are subjected to secondary flow caused by the magnetic force. The secondary flow and thus the mixing efficiency increase as the magnetic flux increases. As the magnetic flux continues to increase, instability occurs at the liquid/liquid interface. Mixing is faster due to instability and the subsequent chaotic flow behaviour in the chamber induced by the magnetic force. Complete mixing between the core and cladding flow occurs almost immediately at a high magnetic flux. The value of mixing efficiency remains constant at its maximum when a critical magnetic flux density is reached. The insets in Figure 6-3 show the images of the mixing chamber at the initial condition, the inception of instability and the completely mixed state. 

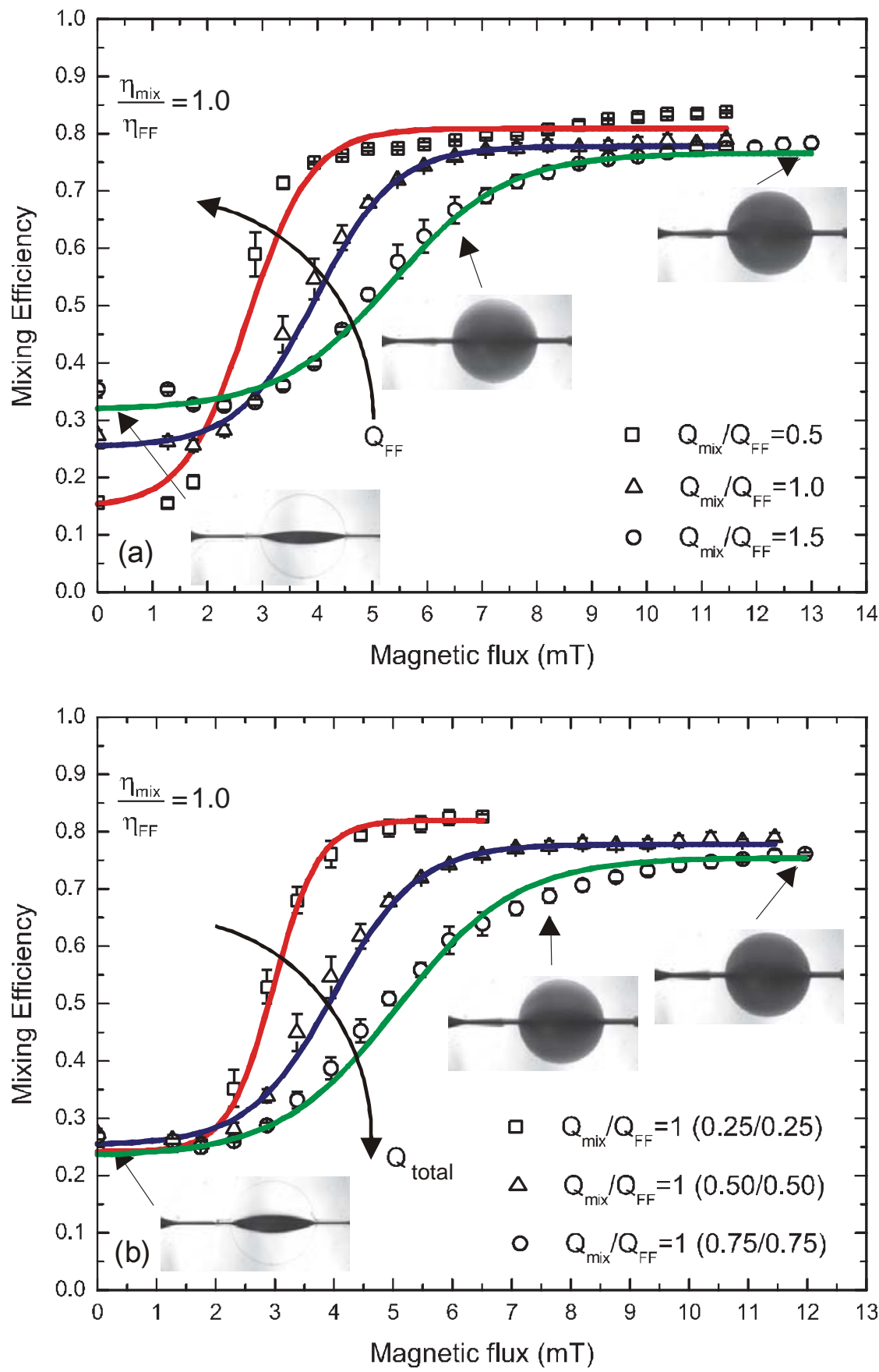

Figure 6-3 Mixing efficiency as a function of magnetic flux density for ferrofluid-core configuration, the viscosity of ferrofluid and water/glycerol mixture are both fixed at $5 \mathrm{mPa} \cdot \mathrm{s}$ : (a) The flow rate of ferrofluid is fixed at $0.5 \mathrm{ml} / \mathrm{h}$ while the flow rate of mixture varies from $0.25 \mathrm{ml} / \mathrm{h}$ to $0.75 \mathrm{ml} / \mathrm{h}$; (b) The flow rates of ferrofluid and mixture vary from $0.25 \mathrm{ml} / \mathrm{h}$ to $0.75 \mathrm{ml} / \mathrm{h}$, but maintain a fixed ratio of 1 . 

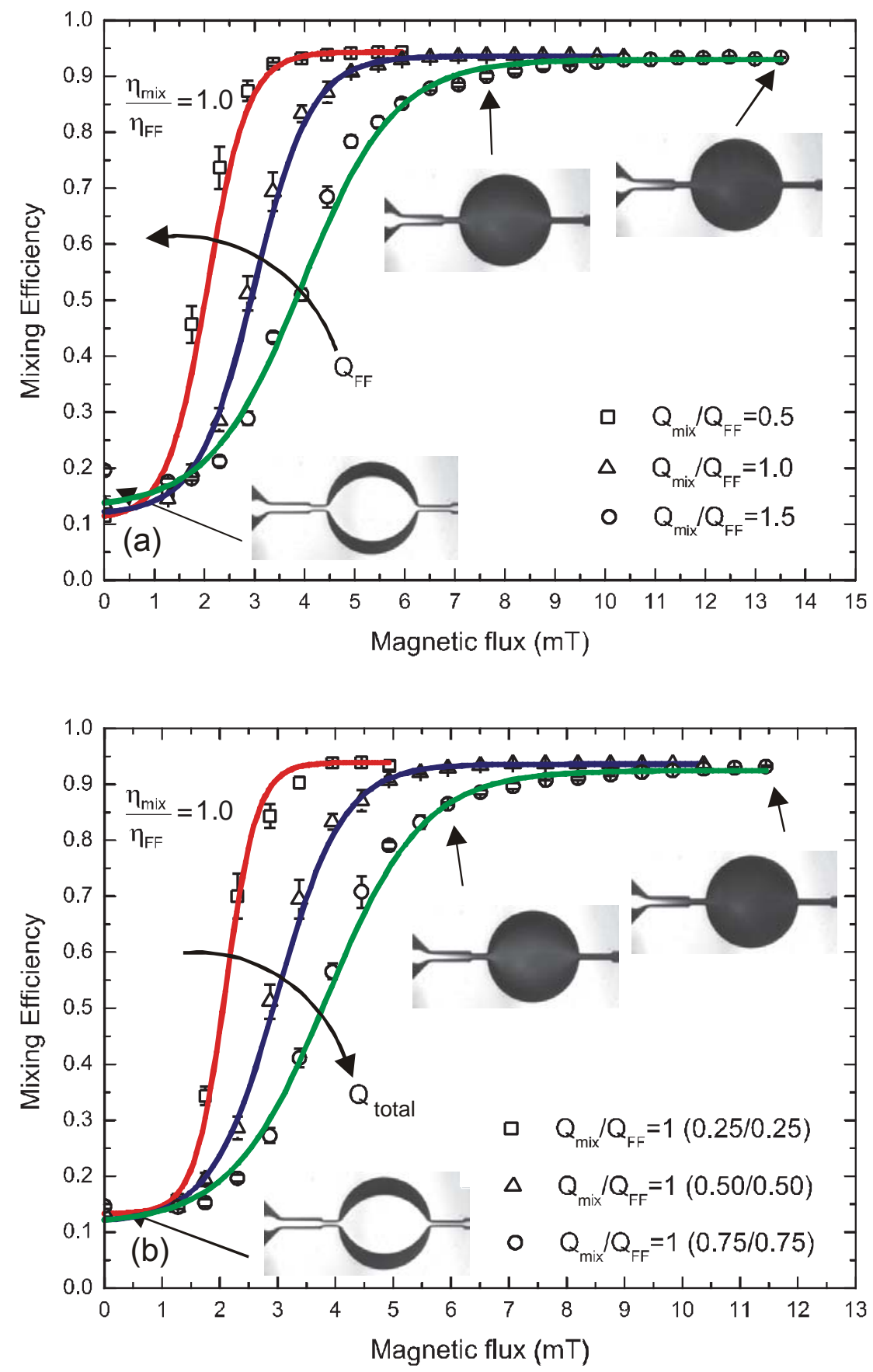

Figure 6-4 Mixing efficiency as a function of magnetic flux density for ferrofluid-cladding configuration, the viscosity of ferrofluid and mixture are fixed at $5 \mathrm{mPa} \cdot \mathrm{s}$ and $5 \mathrm{mPa} \cdot \mathrm{s}$ respectively. (a) The flow rate of ferrofluid is fixed at $0.5 \mathrm{ml} / \mathrm{h}$ while the flow rate of mixture varies from $0.25 \mathrm{ml} / \mathrm{h}$ to $0.75 \mathrm{ml} / \mathrm{h}$; (b) The flow rates of ferrofluid and mixture vary from 0.25 $\mathrm{ml} / \mathrm{h}$ to $0.75 \mathrm{ml} / \mathrm{h}$, but maintain at a fixed ratio of 1 . 

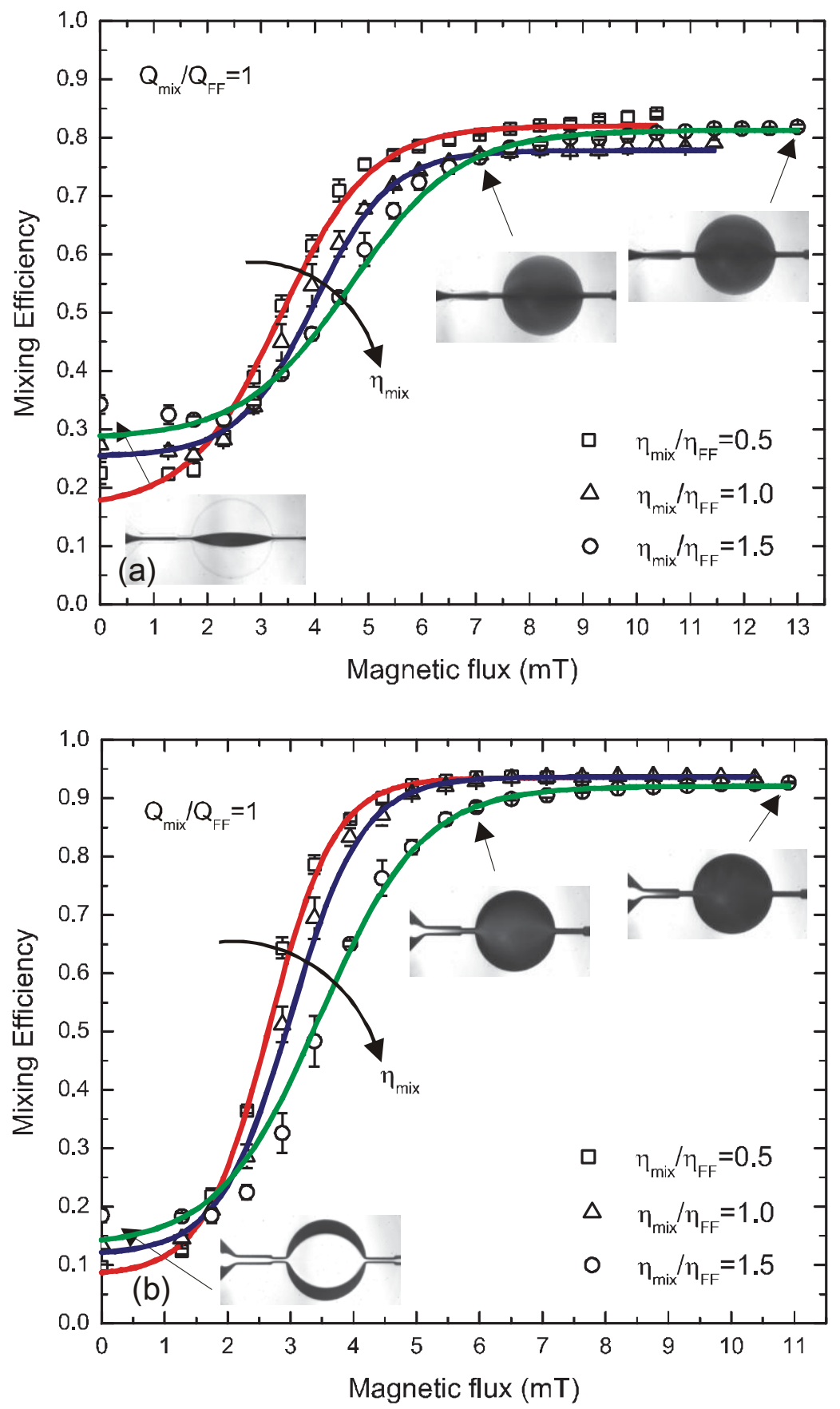

Figure 6-5 Mixing efficiency as a function of magnetic flux density for the same flow rate ratio of 1: (a) Ferrofluid-cladding configuration with viscosity of ferrofluid fixed at $5 \mathrm{mPa} \cdot \mathrm{s}$ while the viscosity of mixture varies from $2.5 \mathrm{mPa} \cdot \mathrm{s}$ to $7.5 \mathrm{mPa} \cdot \mathrm{s}$; (b) Ferrofluid-core configuration with viscosity of ferrofluid fixed at $5 \mathrm{mPa} \cdot \mathrm{s}$ while the viscosity of mixture varies from $2.5 \mathrm{mPa} \cdot \mathrm{s}$ to $7.5 \mathrm{mPa} \cdot \mathrm{s}$.

The amount of ferrofluid in the mixing chamber can be tuned by adjusting the flow rate ratio between the core and the cladding streams. Figure 6-3(a) shows that 
faster mixing and higher efficiency can be achieved with a lower flow rate ratio between cladding and core streams. Because ferrofluid is the active component of the miscible fluid system, the more ferrofluid in the system the more efficient is the mixing process. Due to the initially homogenous distribution of magnetic particles in the ferrofluid, the maximum mixing efficiency is higher with a broader stream of the core flow. Figure 6-3(b) shows how the total flow rate affects the mixing behaviour in the chamber. Faster mixing and a higher mixing efficiency can be achieved at a lower flow rate. Since the mixing concept relies on the competition between magnetically induced secondary flow and the pressure-driven convective flow, the lower the total flow rate the more dominant is the induced secondary flow, and consequently the more efficient is the mixing process. In the case of ferrofluid-core configuration, complete mixing is achieved with mixing efficiency approaching 0.9 .

The results of ferrofluid-cladding configuration with different flow rate ratios and flow rates are shown in Figure 6-4. The mixing process of the ferrofluid-cladding configuration also undergoes the transition from spreading to interface instability to complete mixing. However, the final mixing efficiency is higher than that of the ferrofluid-core case. As shown in the gradient of the efficiency curve, mixing of ferrofluid-cladding configuration is faster and requires a lower magnetic flux density. Similar to the ferrofluid-core case, the same conclusion can be drawn from the results shown in Figure 6-4(a). The more ferrofluid in the system or the lower is the flow rate ratio between the diamagnetic fluid and the ferrofluid, the more efficient is the mixing process. The effect of pressure-driven convective transport over magnetically induced 
transport is also apparent in Figure 6-4(b). A higher magnetic field strength is needed for mixing at a higher total flow rate.

The effect of viscosity ratio on mixing efficiency is analyzed and illustrated in Figure 6-5. The results are presented with the same flow rate of core and cladding streams. Figure 6-5(a) and Figure 6-5(b) compare the effect of viscosity in ferrofluidcore and ferrofluid-cladding experiment, respectively. In both cases, the initial mixing efficiency is lower with smaller viscosity ratio between the diamagnetic fluid and the ferrofluid. However, a lower viscosity of the diamagnetic fluid allows faster mixing and approaches a higher final mixing efficiency. The viscosity of the diamagnetic fluid plays two roles in the mixing process. First, the viscosity affects the migration of magnetic nanoparticles into the diamagnetic fluid. According to Eq. (6.6) the higher the viscosity, the lower is the migration velocity. Second, the viscosity also affects the bulk secondary flow as governed by the Navier-Stokes equation. A higher viscosity means a weaker magnetically induced secondary flow. The combined effect of these two factors leads to the reduction of mixing efficiency with highly viscous diamagnetic fluid. 


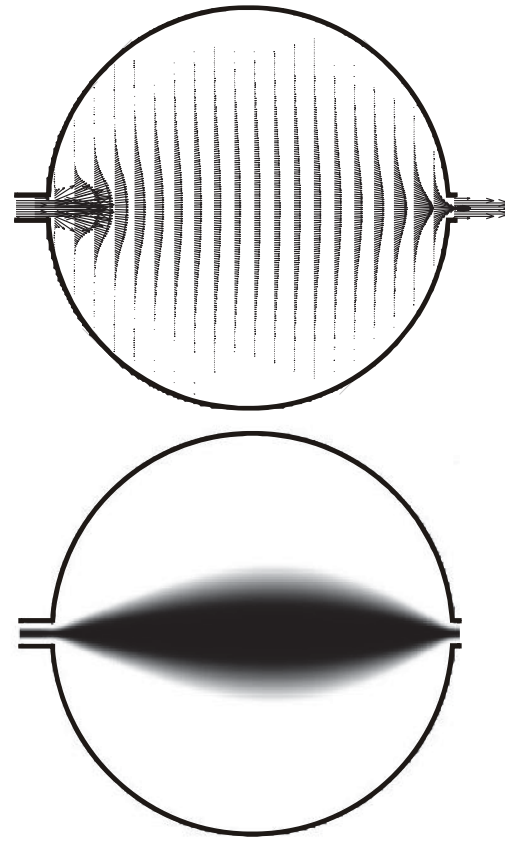

(a) $\mathrm{B}=0 \mathrm{mT}$
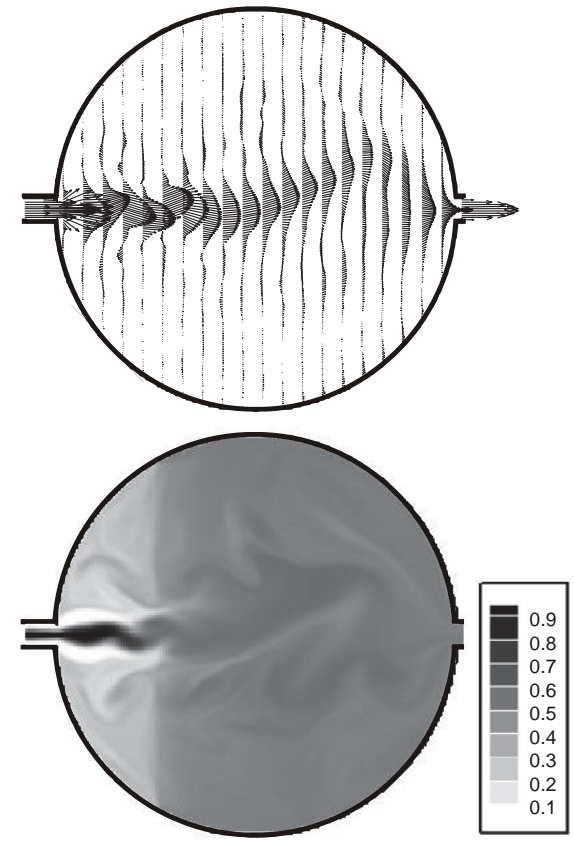

(b) $\mathrm{B}=9.3 \mathrm{mT}$

Figure 6-6 Simulated velocity and concentration fields of ferrofluid-core configuration: (a) no magnetic field; (b) with magnetic field.

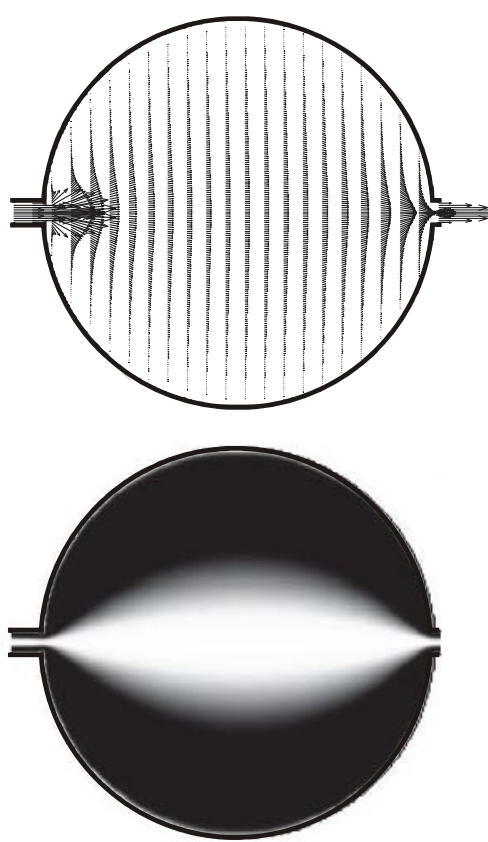

(a) $\mathrm{B}=0 \mathrm{mT}$
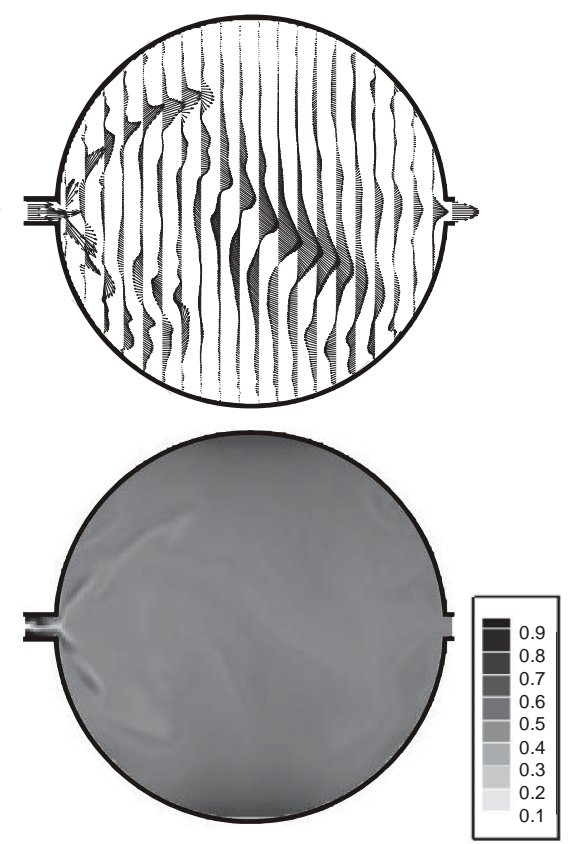

(b) $\mathrm{B}=9.3 \mathrm{mT}$

Figure 6-7 Simulated velocity and concentration fields of ferrofluid-cladding configuration: (a) no magnetic field; (b) with magnetic field. 


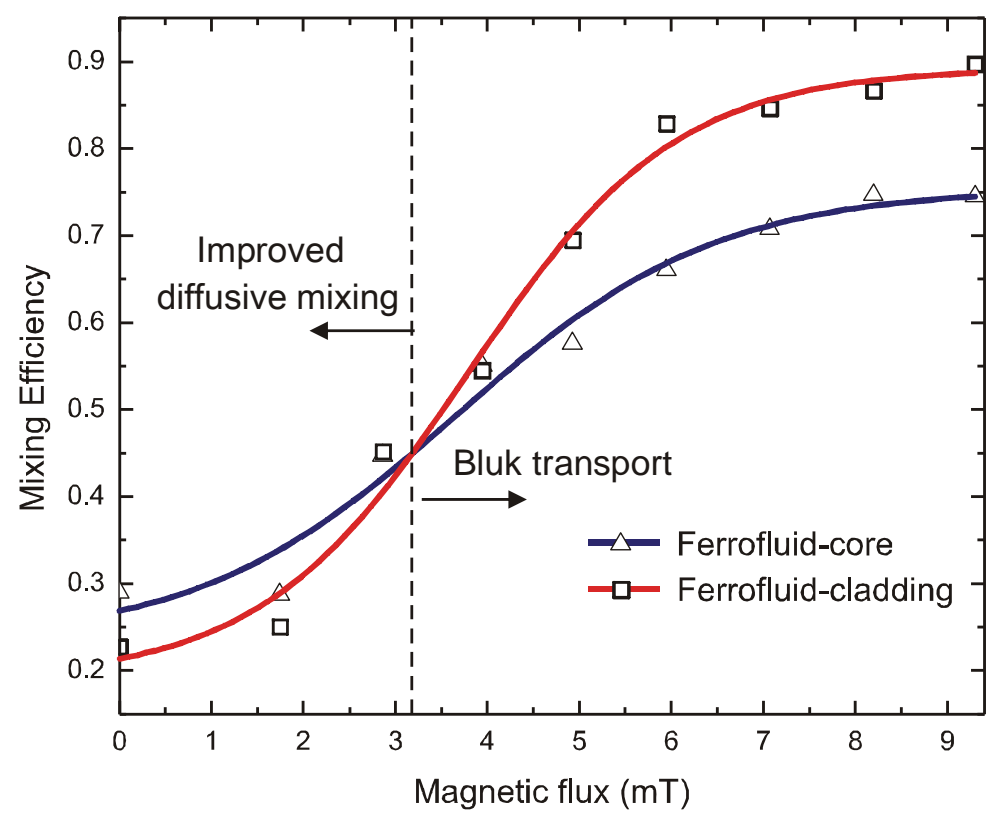

Figure 6-8 Numerically simulated mixing efficiency at the different magnetic flux densities for both the ferrofluid-core and ferrofluid-cladding configurations.

As described in the previous section, numerical simulation was implemented for both ferrofluid-core and ferrofluid-cladding configurations. The viscosity ratio between the diamagnetic fluid and the ferrofluid was fixed at 0.5 . The flow rates of both fluids were fixed at $0.5 \mathrm{ml} / \mathrm{h}$. The present numerical simulation aimed at providing a qualitative understanding of the mixing behaviour. Thus, the effects of viscosity and flow rate variation on the mixing behaviour were not investigated numerically. In the simulation, the ferrofluid was assumed to be linearly magnetized. The grid independent study was first performed to find a compromise between grid size and accuracy. Calculation error could be reduced with further grid refinement as the numerical diffusion was significantly affected by the grid size. A grid size of $200 \times 182$ was finally selected in the simulation, because numerical diffusion could not be considerably reduced with further grid refinement. 
Figure 6-6 and Figure 6-7 show the representative results of the velocity and concentration fields of the ferrofluid-core and ferrofluid-cladding configurations, respectively. Without a magnetic field, the pressure-driven flow follows stable paths from the inlet to the exit of the chamber. Mixing is caused purely by molecular diffusion. As mentioned in the previous section, the excessive diffusion observed in Figure 6-6(a) and Figure 6-7(a) is actually caused by the numerical calculation. A sharper interface is observed in the experiments due to the high Peclet numbers involved. The existence of a magnetically induced secondary flow is apparent in the simulated results of the velocity field shown in Figure 6-6(b) and Figure 6-7(b). Fast mixing is achieved by the chaotic secondary flow induced by the mismatch of magnetic susceptibilities. The numerical results of mixing efficiency are presented in Figure 6-8 with the same Boltzmann fitting function used for experimental data. The mixing efficiency curves show a similar trend as the experimental results. The mixing process can basically be divided into two regimes. At low magnetic field strengths, mixing is caused by improved migration of magnetic particles into the diamagnetic fluid. The applied uniform magnetic field leads to a higher apparent diffusion coefficient of the particle. The mixing process behaves similar to purely molecular diffusive mixing [206]. At high magnetic field strengths, the effect of magnetically induced secondary flow becomes apparent. Mixing occurs rapidly due to the more effective transport of the bulk fluid. In agreement with the experiment, the ferrofluidcladding configuration leads to a higher final mixing efficiency. 

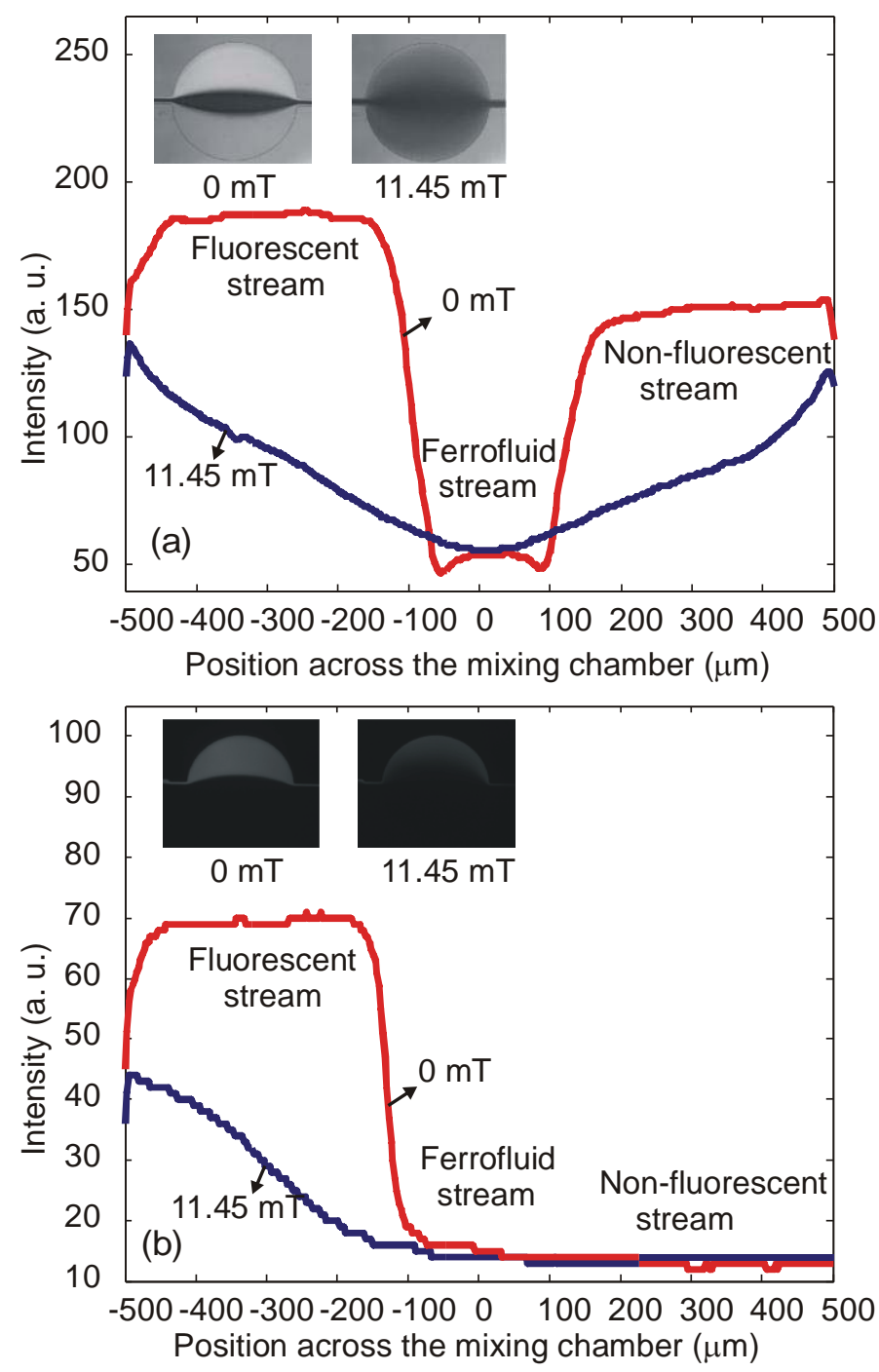

Figure 6-9 Mixing of a three-fluids system consisting of fluorescent diamagnetic fluid, ferrofluid and non-fluorescent diamagnetic fluid: (a) Intensity profile across the mixing chamber in a bright field image with epi-fluorescent filter; (b) Intensity profile in a fluorescent image with epi-fluorescent filter only.

The present work only focuses on mixing and the concentration distribution of magnetic particles in the ferrofluid. The above insights gained from the numerical simulation show that in the first regime of "improved diffusive mixing" only magnetic particles migrate into the diamagnetic fluid. Solutes in the diamagnetic fluid will not be affected in this regime. Only in the second regime, effective mixing of solutes in the diamagnetic fluid can be achieved due to the bulk transport of the magnetically 
induced secondary flow. However, it's difficult to evaluate the mixing efficiency of a solute optically, because magnetic particles and the ferrofluid are opaque and block both excitation and emission light to and from a fluorescent solute. To verify this point, we carried out the mixing experiment with the ferrofluid-core configuration using a three-fluids system: fluorescent diamagnetic fluid, ferrofluid and non-fluorescent diamagnetic fluid. All fluids have the same viscosity. The flow rates of the cladding diamagnetic fluids are half of that of the ferrofluid core, so that the total flow rate ratio between diamagnetic fluid and ferrofluid is unity. As the fluorescent fluid, the mixture prepared for the previous experiments has a viscosity matched that if the ferrofluid was mixed with a fluorescent dye (fluorescein disodium salt C20H10Na2O5) [207]. The dye is also called Acid Yellow 73 or C.I. 45350. Figure 6-9 shows the intensity profile across the mixing chamber for both cases with and without magnetic field. The images were recorded with both bright through light and an epi-fluorescent filter (Nikon B-2A, excitation filter for $450-490 \mathrm{~nm}$, dichroic mirror for $505 \mathrm{~nm}$ and an emission filter for $520 \mathrm{~nm}$ ), so that the fluorescent and the non-fluorescent diamagnetic fluids could be discriminated. Figure 6-9(a) shows a similar intensity profile across both fluid indicating that magnetic particles has migrated into the diamagnetic fluids. However, it cannot be concluded that dye molecules is transported to the other non-fluorescent side of the chamber. Figure 6-9(b) shows the same profiles of the images using the epi-fluorescent filter only. A slight increase in intensity value on the non-fluorescent side can be observed. However, the blocking effect of the magnetic particles is still dominant. 


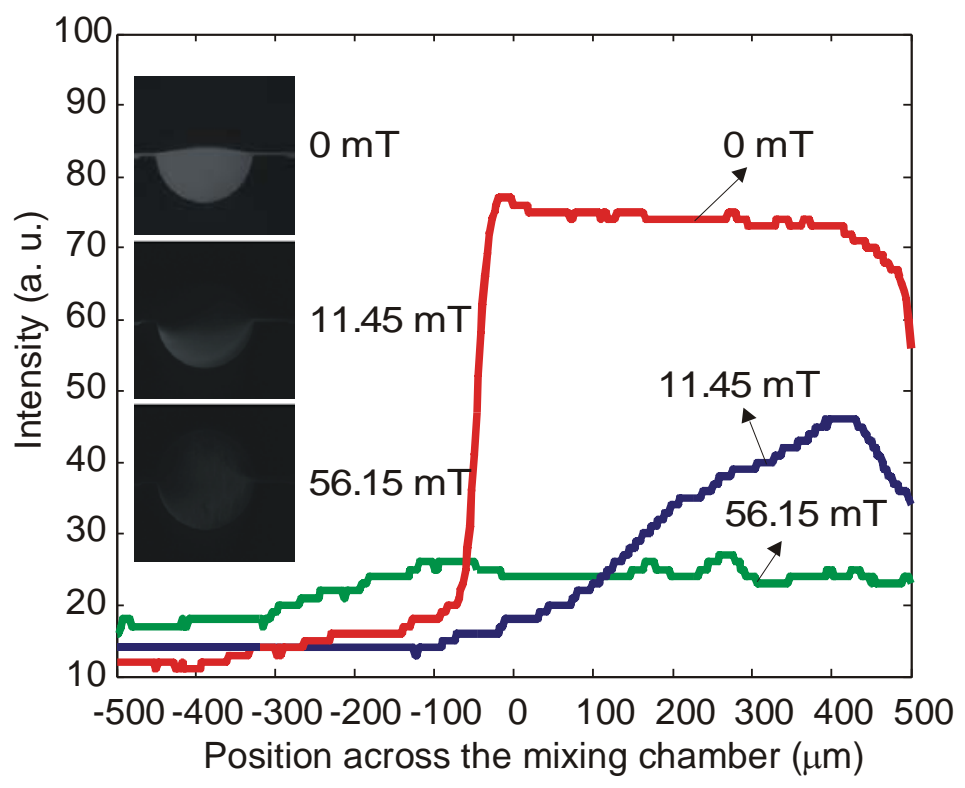

Figure 6-10 Intensity profile across the mixing chamber with a two-fluids system consisting of a ferrofluid and a fluorescent diamagnetic fluid.

The effectiveness of mixing the fluorescent solute in the experiment of Figure 6-9 is not apparent, and may be caused by the symmetric arrangement of the streams. The fluorescent dye may mix well with the ferrofluid in the upper half of the chamber but does not cross to the lower half due to symmetry. To verify this point, we designed another experiment with an asymmetric arrangement of a two-fluids system: ferrofluid and fluorescent diamagnetic fluid. The results of the intensity profile and the corresponding fluorescent images are depicted in Figure 6-10. Similar to the experiment of Figure 6-9, a slight increase in intensity of the ferrofluid side can be observed. At a much higher flux density of $56.15 \mathrm{mT}$ with an applied current of $2 \mathrm{~A}$, clear secondary flow pattern can be observed and the magnetic particles agglomerate and form large clusters due to dipole interactions. The reduction of the concentration magnetic particles elsewhere, allowing excitation light to reach the dye and emission light to escape. A relatively uniform intensity profile with increased intensity values is 
the proof that the fluorescent solute molecules spread across the entire mixing chamber.

\subsection{Summary}

Mixing in a circular chamber was realized with a ferrofluid under a uniform external magnetic field. The microfluidic device used in the experiment offers a laminar flow condition with negligible molecular diffusion. The uniform magnetic field is employed to generate instability at the interface of the liquids with mismatched magnetic susceptibilities and consequently a gradient in magnetization. Mixing is instantaneous at a relatively low magnetic field of less than $10 \mathrm{mT}$. The maximum mixing efficiency is around $90 \%$. The experimental results show that the mixing process is affected by factors such as flow rate ratio, flow rate and viscosity. The mixing process consists of two basic regimes. The first regime under low applied field strength is similar to molecular diffusive mixing caused by the improved migration of magnetic particles into the diamagnetic fluid. The second regime is bulk transport mixing caused by magnetically induced secondary flow. Between these two regimes is a transition region of unstable interface between the two fluids. The numerical simulation incorporates the underlying physics of the mixing process and can qualitatively explain the experimental data. However, the numerical diffusion considerably affects the simulation results. To obtain more accurate and quantitative simulation results, the problem of numerical diffusion needs to be addressed in the future works. 


\section{CHAPTER 7}

\section{CONCLUSIONS AND FUTURE WORK}

\subsection{Conclusions}

In this study, the realization of interaction between a magnetic field and fluid flow has been achieved in both digital and continuous microfluidic systems. In digital microfluidics, the ferrofluid droplet behaviour has been investigated under the effect of gravity, interfacial tension force, and an external magnetic field. The wetting property, as well as activated motion, has been studied with the ferrofluid droplet on a hydrophobic glass slide. In continuous microfluidics, microfluidic devices for spreading and mixing have been designed and developed. With ferrofluid acting as core and cladding stream in a three-stream flow system, spreading of magnetic nanoparticles and occurring of interface instability in a uniform magnetic field has been utilized. Following are the main conclusions drawn from the study.

(1) The experiment has been conducted to show that a ferrofluid droplet is stretched in the direction of a uniform magnetic field. The horizontal magnetic field results in an increase in the width and decrease in the height of the sessile ferrofluid droplet. A numerical model is established for further understanding of this magnetic field induced phenomena. The magnetization of the ferrofluid is taken to be nonlinear. Comparison is carried out between the numerical prediction and experimental investigation under the effect of interfacial tension, gravitational and magnetic forces. The measured droplet shapes agree well with the simulations at different 
magnetic Bond number.

(2) It is demonstrated that nonuniform magnetic field contributes to both wettability and motion of ferrofluid droplet. It is found that under the effect of pulling down and laterally stretching due to magnetic field, the base diameter of the ferrofluid droplet increases almost linearly with the flux density, while its height tends to reach an asymptotic value. A scaling analysis is conducted for the droplet inception through establishing force balance. Experimental results also help to estimate the value of factors. The critical velocity for droplet sliding can be obtained under magnetic field with different flux density.

(3) Experimental and numerical investigations have been carried out to study the phenomenon of magnetofluidic spreading in a uniform magnetic field. The magnetofluidic spreading effect is investigated for both ferrofluid-core and ferrofluid-cladding case. The width of the core stream is evaluated as a function of the applied magnetic flux density. A stronger spreading effect can be observed at larger magnetic field strength. The numerical simulation shows that magnetofluidic spreading is caused by migration of magnetic particles and secondary flow at the ferrofluid/oil interface. Both phenomena are caused by a susceptibility gradient or the concentration gradient of magnetic particles in the ferrofluid. Both experiment and simulation show a similar behaviour of the flow system.

(4) By using a uniform magnetic field, mixing in a circular chamber has been realized with a ferrofluid in the present study. In the microfluidic device, the molecular 
diffusion is negligible as the fluid flows in a laminar way. For both ferrofluid-core and ferrofluid-cladding case, the magnetic force depends on the gradient of magnetization due to the mismatched magnetic susceptibilities at the interface. The volumetric magnetic force induces instability at the interface of the liquids. The mixing is instantaneous at a relatively low magnetic field less than $10 \mathrm{mT}$. The maximum mixing efficiency is around $90 \%$. Important factors such as flow rate ratio, flow rate and viscosity have been investigated in the experiments. The mixing process consists of two basic regimes: improved diffusive mixing and bulk transport. The first regime is similar to molecular diffusion caused by the improved migration of magnetic particles into the diamagnetic fluid. Under low field strength, the magnetic particles are driven by the magnetic force on individual particle. The volumetric magnetic force is not large enough to affect the flow field. The second regime is bulk transport mixing caused by magnetically induced secondary flow. Between these two regimes is a transition region of unstable interface between the two fluids. The numerical simulation which can qualitatively explain the experimental data has been implemented.

\subsection{Future Works}

With the success of studying wetting and motion of ferrofluid droplet under uniform and non-uniform magnetic field, as well as the spreading and mixing of ferrofluid in a uniform field, it has paved the way to carry on the further investigations for magnetic application in many microfluidic devices. 
(1) Experimentally investigating sessile droplets coalescence under the driving force of a non-uniform magnetic field in digital microfluidics. A ferrofluid droplet can be used as the driving component. It has the potential applications in chemical and biochemical area. By using fluorescent dye for visualization, analysis of the experimental results can provide detailed information on mixing pattern and intensity distribution. Developing a numerical model for transport of magnetic liquid droplets driven by a permanent magnetic field. The difficulty includes the moving contact line and non-uniformly distributed magnetic field.

(2) New configuration of magnetic field such as vertical field direction, rotational field etc. to study wetting or kinematics of the ferrofluid droplet. The study on ferrofluid droplet in a vertical magnetic field offers an easier estimation on the magnitudes of the magnetic force. The investigation on ferrofluid droplet in a rotational magnetic field helps in optimization of magnetic pumping method.

(3) Computational schemes to overcome numerical diffusion need to be considered to have quantitative results, because the interaction between magnetic field and the flow field is extremely sensitive to the gradient of particle concentration. 


\section{List of Publication}

[1] Nguyen, N. T.; Zhu, G.P.; Chua, Y. C.; Phan, V. N.; Tan, S. H., Magnetowetting and sliding motion of a sessile ferrofluid droplet in the presence of a permanent magnet. Langmuir 2010, 26, (15), 12553-12559.

[2] G.P. Zhu, N.T. Nguyen Particle Sorting in Microfluidic Systems, Micro and Nanosystems 2010, Vol. 2, No. 3, pp. 202-216.

[3] G.P. Zhu; N.T. Nguyen; R.V. Ramanujan; X.Y. Huang Deformation of a ferrofluid droplet in a uniform magnetic field. Langmuir, Vol. 27, No. 24, 2011, pp. 1483414841.

[4] G.P. Zhu; N.T. Nguyen, Magnetofluidic spreading in microchannels, Microfluidics and Nanofluidics, Vol. 13, No. 4, 2012, pp 655-663.

[5] G.P. Zhu; N.T. Nguyen, Rapid magnetofluidic mixing in a uniform magnetic field, Lab on a Chip, Vol. 12, No. 22, 2012.,pp. 4772-4780.

[6] G.P. Zhu; N.T. Nguyen, Transport of magnetic particles under an uniform magnetic field in microchannels, Proceedings of the 11th International Conference on Nanochannels, Microchannels, and Minichannels, ICNMM Accepted, 2013. 


\section{References}

1. Nguyen, N.T., Micro-magnetofluidics: interactions between magnetism and fluid flow on the microscale. Microfluidics and Nanofluidics, 2012: p. 1-16.

2. Ritchie, W., Experimental researches in voltaic electricity and electro-magnetism. Philosophical Transactions of the Royal Society of London, 1832. 122: p. 279-298.

3. Davidson, P.A., An Introduction to Magnetohydrodynamics. 2001: University of Cambridge.

4. Qian, S. and H.H. Bau, Magneto-hydrodynamics based microfluidics. Mechanics Research Communications, 2009. 36(1): p. 10-21.

5. Zhong, J., M. Yi, and H.H. Bau, Magneto hydrodynamic (MHD) pump fabricated with ceramic tapes. Sensors and Actuators A: Physical, 2002. 96(1): p. 59-66.

6. Lee, J., et al., Influence of magnetic fields on the voltammetric response of microelectrodes in highly concentrated organic redox solutions. Journal of the Electrochemical Society, 1995. 142(6): p. L90-L92.

7. Lee, J., et al., Magnetic field control of the potential distribution and current at microdisk electrodes. Journal of Electroanalytical Chemistry, 1997. 422(1): p. 169177.

8. Jang, J. and S.S. Lee, Theoretical and experimental study of MHD (magnetohydrodynamic) micropump. Sensors and Actuators A: Physical, 2000. 80(1): p. 84-89.

9. Huang, L., et al., LIGA fabrication and test of a DC type magnetohydrodynamic (MHD) micropump. Microsystem technologies, 2000. 6(6): p. 235-240.

10. Ghaddar, N. and E. Sawaya, Testing and Modeling Thermosyphonic Closed-Loop Magnetohydrodynamic Electrolyte Flow. Journal of Thermophysics and Heat Transfer, 2003. 17(2): p. 129-137.

11. Sawaya, E., N. Ghaddar, and F. Chaaban, Evaluation of the Hall parameter of electrolyte solutions in thermosyphonic MHD flow. International Journal of Engineering Science, 2002. 40(18): p. 2041-2056.

12. Homsy, A., et al., A high current density DC magnetohydrodynamic (MHD) micropump. Lab on a Chip, 2005. 5(4): p. 466-471.

13. Homsy, A., et al., Magnetohydrodynamic pumping in nuclear magnetic resonance environments. Sensors and Actuators B: Chemical, 2007. 123(1): p. 636-646.

14. Nguyen, B. and S.K. Kassegne, High-current density DC magenetohydrodynamics micropump with bubble isolation and release system. Microfluidics and Nanofluidics, 2008. 5(3): p. 383-393.

15. Ragsdale, S.R., et al., Magnetic field effects in electrochemistry. Voltammetric reduction of acetophenone at microdisk electrodes. The Journal of Physical Chemistry, 1996. 100(14): p. 5913-5922.

16. Ragsdale, S.R., K.M. Grant, and H.S. White, Electrochemically generated magnetic forces. Enhanced transport of a paramagnetic RedOx species in large, nonuniform magnetic fields. Journal of the American Chemical Society, 1998. 120(51): p. 1346113468 . 
17. Leventis, N. and X. Gao, Steady-state voltammetry with stationary disk millielectrodes in magnetic fields: nonlinear dependence of the mass-transfer limited current on the electron balance of the faradaic process. The Journal of Physical Chemistry B, 1999. 103(28): p. 5832-5840.

18. Pullins, M.D., K.M. Grant, and H.S. White, Microscale confinement of paramagnetic molecules in magnetic field gradients surrounding ferromagnetic microelectrodes. The Journal of Physical Chemistry B, 2001. 105(37): p. 8989-8994.

19. Mohanta, S. and T. Fahidy, Mass transfer in cylindrical magnetoelectrolytic cells. Electrochimica Acta, 1976. 21(2): p. 149-152.

20. Mori, S., K. Satoh, and A. Tanimoto, Electrolytic mass transfer around inclined cylinders in static magnetic fields. Electrochimica acta, 1994. 39(18): p. 2789-2794.

21. Mohanta, S. and T. Fahidy, Mass transfer in a magnetoelectrolytic flow cell. Electrochimica Acta, 1974. 19(12): p. 835-840.

22. Leventis, N., et al., Electrochemistry with stationary disk and ring-disk millielectrodes in magnetic fields. The Journal of Physical Chemistry B, 1998. 102(18): p. 3512-3522.

23. Arumugam, P.U., et al., Characterization and pumping RedOx magnetohydrodynamics in a microfluidic channel. Journal of The Electrochemical Society, 2006. 153(12): p. E185-E194.

24. Grant, K.M., J.W. Hemmert, and H.S. White, Magnetic field-controlled microfluidic transport. Journal of the American Chemical Society, 2002. 124(3): p. 462-467.

25. Duwairi, H. and M. Abdullah, Thermal and flow analysis of a magneto-hydrodynamic micropump. Microsystem technologies, 2007. 13(1): p. 33-39.

26. Ngo Boum, G. and A. Alemany, Numerical simulations of electrochemical mass transfer in electromagnetically forced channel flows. Electrochimica Acta, 1999. 44(11): p. 1749-1760.

27. Qian, S. and H.H. Bau, Magnetohydrodynamic flow of RedOx electrolyte. Departmental Papers (MEAM), 2005: p. 107.

28. Kabbani, H., et al., Modeling RedOx-based magnetohydrodynamics in threedimensional microfluidic channels. Physics of Fluids, 2007. 19: p. 083604.

29. Aguilar, Z.P., P. Arumugam, and I. Fritsch, Study of magnetohydrodynamic driven flow through LTCC channel with self-contained electrodes. Journal of Electroanalytical Chemistry, 2006. 591(2): p. 201-209.

30. Ho, J.E., Characteristic study of MHD pump with channel in rectangular ducts. Journal of Marine Science and Technology, 2007. 15(4): p. 315-321.

31. Patel, V. and S.K. Kassegne, Electroosmosis and thermal effects in magnetohydrodynamic (MHD) micropumps using 3D MHD equations. Sensors and Actuators B: Chemical, 2007. 122(1): p. 42-52.

32. Lemoff, A.V. and A.P. Lee, An AC magnetohydrodynamic micropump. Sensors and Actuators B: Chemical, 2000. 63(3): p. 178-185.

33. Wang, P.J., C.Y. Chang, and M.L. Chang, Simulation of two-dimensional fully developed laminar flow for a magneto-hydrodynamic (MHD) pump. Biosensors and Bioelectronics, 2004. 20(1): p. 115-121. 
34. Affanni, A. and G. Chiorboli. Numerical Modelling and Experimental Study of an AC Magnetohydrodynamic (MHD) Micropump. in Instrumentation and Measurement Technology Conference, 2006. IMTC 2006. Proceedings of the IEEE. 2006: IEEE.

35. Sadler, D.J., et al. Ceramic magnetohydrodynamic (MHD) micropump. in Micromachining and Microfabrication. 2001: International Society for Optics and Photonics.

36. Gleeson, J.P. and J. West. Magnetohydrodynamic micromixing. in Proceedings of the Fifth International Conference on Modeling and Simulation of Microsystems. 2002.

37. West, J., et al., Structuring laminar flows using annular magnetohydrodynamic actuation. Sensors and Actuators B: Chemical, 2003. 96(1-2): p. 190-199.

38. West, J., et al., Application of magnetohydrodynamic actuation to continuous flow chemistry. Lab on a Chip, 2002. 2(4): p. 224-230.

39. Eijkel, J.C.T., et al., A circular ac magnetohydrodynamic micropump for chromatographic applications. Sensors and Actuators B: Chemical, 2003. 92(1-2): p. 215-221.

40. Eijkel, J.C.T., A. van den Berg, and A. Manz, Cyclic electrophoretic and chromatographic separation methods. Electrophoresis, 2004. 25(2): p. 243-252.

41. Heng, K.-H., et al., UV-LIGA microfabrication and test of an ac-type micropump based on the magnetohydrodynamic (MHD) principle. 2000: p. 161-171.

42. Heng, K.-H., et al., Development of a diffuser/nozzle-type micropump based on magnetohydrodynamic (MHD) principle. 1999: p. 66-73.

43. Gleeson, J.P., et al., Modelling annular micromixers. SIAM Journal on Applied Mathematics, 2004: p. 1294-1310.

44. Gleeson, J.P., Transient micromixing: Examples of laminar and chaotic stirring. Physics of Fluids, 2005. 17: p. 100614.

45. Bau, H.H., J. Zhong, and M. Yi, A minute magneto hydro dynamic (MHD) mixer. Sensors and Actuators B: Chemical, 2001. 79(2): p. 207-215.

46. Xiang, Y. and H.H. Bau, Complex magnetohydrodynamic low-Reynolds-number flows. Physical Review E, 2003. 68(1): p. 016312.

47. Yi, M., S. Qian, and H.H. Bau, A magnetohydrodynamic chaotic stirrer. Journal of Fluid Mechanics, 2002. 468: p. 153-177.

48. Qian, S. and H.H. Bau, Magneto-hydrodynamic stirrer for stationary and moving fluids. Sensors and Actuators B: Chemical, 2005. 106(2): p. 859-870.

49. Bau, H.H., et al., A magneto-hydrodynamically controlled fluidic network. Sensors and Actuators B: Chemical, 2003. 88(2): p. 205-216.

50. Qian, S., J. Zhu, and H.H. Bau, A stirrer for magnetohydrodynamically controlled minute fluidic networks. Physics of Fluids, 2002. 14: p. 3584.

51. Lemoff, A.V. and A.P. Lee, An ac magnetohydrodynamic microfluidic switch for micro total analysis systems. Biomedical Microdevices, 2003. 5(1): p. 55-60.

52. Pankhurst, Q.A., et al., Applications of magnetic nanoparticles in biomedicine. Journal of Physics D: Applied Physics, 2003. 36: p. R167.

53. Matsunaga, T. and S. Kamiya, Use of magnetic particles isolated from magnetotactic bacteria for enzyme immobilization. Applied Microbiology and Biotechnology, 1987. 26(4): p. 328-332. 
54. Dressman, D., et al., Transforming single DNA molecules into fluorescent magnetic particles for detection and enumeration of genetic variations. Proceedings of the National Academy of Sciences, 2003. 100(15): p. 8817.

55. Yoza, B., M. Matsumoto, and T. Matsunaga, DNA extraction using modified bacterial magnetic particles in the presence of amino silane compound. Journal of Biotechnology, 2002. 94(3): p. 217-224.

56. Rida, A. and M. Gijs, Manipulation of self-assembled structures of magnetic beads for microfluidic mixing and assaying. Analytical chemistry, 2004. 76(21): p. 6239-6246.

57. Astalan, A.P., et al., Biomolecular reactions studied using changes in Brownian rotation dynamics of magnetic particles. Biosensors and Bioelectronics, 2004. 19(8): p. 945-951.

58. Shinkai, M., Functional magnetic particles for medical application. Journal of Bioscience and Bioengineering, 2002. 94(6): p. 606-613.

59. Korneva, G., et al., Carbon nanotubes loaded with magnetic particles. Nano Letters, 2005. 5(5): p. 879-884.

60. Jensen, K. and A. Lee, Lab on a Chip - Miniaturisation for Chemistry and Biology: Special issue foreword. Lab on a Chip - Miniaturisation for Chemistry and Biology, 2004. 4(4): p. $31 \mathrm{~N}-32 \mathrm{~N}$.

61. Bikerman, J., Sliding of drops from surfaces of different roughnesses. Journal of colloid science, 1950. 5(4): p. 349-359.

62. Hyväluoma, J., et al., Droplets on inclined rough surfaces. The European Physical Journal E: Soft Matter and Biological Physics, 2007. 23(3): p. 289-293.

63. Berthier, J., microdrops and digital microfluidics. 2008, New York.

64. Chaudhury, M.K. and G.M. Whitesides, How to make water run uphill. Science, 1992. 256(5063): p. 1539-1544.

65. Young, N., J. Goldstein, and M. Block, The motion of bubbles in a vertical temperature gradient. Journal of Fluid Mechanics, 1959. 6(03): p. 350-356.

66. Inaki, Y., Thymine Polymers as High Resolution Photoresists and Reversible PhotoRecording Materials. Polymer News, 1992. 17: p. 367-367.

67. Lee, J. and C.J. Kim, Surface-tension-driven microactuation based on continuous electrowetting. Journal of Microelectromechanical Systems, 2002. 9(2): p. 171-180.

68. Gascoyne, P.R.C., et al., Dielectrophoresis-based programmable fluidic processors. Lab on a Chip, 2004. 4(4): p. 299-309.

69. Wixforth, A., Acoustically driven planar microfluidics. Superlattices and Microstructures, 2003. 33(5-6): p. 389-396.

70. Guttenberg, Z., et al., Planar chip device for PCR and hybridization with surface acoustic wave pump. Lab Chip, 2005. 5(3): p. 308-17.

71. Long, Z., et al., Fundamentals of magnet-actuated droplet manipulation on an open hydrophobic surface. Lab on a Chip - Miniaturisation for Chemistry and Biology, 2009. 9(11): p. 1567-1575.

72. Rosensweig, R.E., Ferrohydrodynamics. 1985, New York: Cambridge University Press.

73. Odenbach, S., Magnetoviscous and viscoelastic effects in ferrofluids. International Journal of Modern Physics B, 2000. 14(16): p. 1615-1632. 
74. Yang, C.G., Z.R. Xu, and J.H. Wang, Manipulation of droplets in microfluidic systems. TrAC Trends in Analytical Chemistry, 2010. 29(2): p. 141-157.

75. Zheng, L. and H. Zhang, An adaptive level set method for moving-boundary problems: application to droplet spreading and solidification. Numerical Heat Transfer Part B: Fundamentals, 2000. 37(4): p. 437-454.

76. Spelt, P.D.M., A level-set approach for simulations of flows with multiple moving contact lines with hysteresis. Journal of Computational Physics, 2005. 207(2): p. 389404.

77. Spelt, P.D.M., Shear flow past two-dimensional droplets pinned or moving on an adhering channel wall at moderate Reynolds numbers: a numerical study. Journal of Fluid Mechanics, 2006. 561(-1): p. 439-463.

78. Tryggvason, G., et al., A front-tracking method for the computations of multiphase flow. Journal of Computational Physics, 2001. 169(2): p. 708-759.

79. Yap, Y.F., et al., Thermally mediated control of liquid microdroplets at a bifurcation. Journal of Physics D: Applied Physics, 2009. 42: p. 065503.

80. Sero-Guillaume, O.E., et al., The shape of a magnetic liquid drop. Journal of Fluid Mechanics, 1992. 241: p. 215-232.

81. Lavrova, O., et al., Numerical treatment of free surface problems in ferrohydrodynamics. Journal of Physics: Condensed Matter, 2006. 18: p. S2657.

82. Shikida, M., et al., Development of an enzymatic reaction device using magnetic beadcluster handling. Journal of Micromechanics and Microengineering, 2006. 16(9): p. 1875-1883.

83. Schneider, J., et al., Motion of viscous drops on superhydrophobic surfaces due to magnetic gradients. Colloids and Surfaces A: Physicochemical and Engineering Aspects, 2008. 323(1-3): p. 19-27.

84. García, A.A., et al., Magnetic movement of biological fluid droplets. Journal of Magnetism and Magnetic Materials, 2007. 311(1 SPEC. ISS.): p. 238-243.

85. Pipper, J., et al., Clockwork PCR including sample preparation. Angewandte Chemie - International Edition, 2008. 47(21): p. 3900-3904.

86. Shikida, M., et al., Using wettability and interfacial tension to handle droplets of magnetic beads in a micro-chemical-analysis system. Sensors and Actuators, B: Chemical, 2006. 113(1): p. 563-569.

87. Vékás, L., D. Bica, and M.V. Avdeev, Magnetic nanoparticles and concentrated magnetic nanofluids: Synthesis, properties and some applications. China Particuology, 2007. 5(1-2): p. 43-49.

88. Gerber, R., M. Takayasu, and F. Friedlaender, Generalization of HGMS theory: the capture of ultra-fine particles. Magnetics, IEEE Transactions on, 1983. 19(5): p. 2115-2117.

89. Morozov, K. and A. Lebedev, The effect of magneto-dipole interactions on the magnetization curves of ferrocolloids. Journal of Magnetism and Magnetic Materials, 1990. 85(1-3): p. 51-53.

90. Wertheim, M.S., Exact solution of the mean spherical model for fluids of hard spheres with permanent electric dipole moments. The Journal of Chemical Physics, 1971. 55(9): p. 4291-4298. 
91. Odenbach, S., Magnetoviscous effects in ferrofluids. Applied Rheology, 2000. 10(4): p. 178-184.

92. McTague, J.P., Magnetoviscosity of magnetic colloids. The Journal of Chemical Physics, 1969. 51(50): p. 133-136.

93. Elmore, W.C., The magnetization of ferromagnetic colloids. Physical Review, 1938. 54(12): p. 1092-1095.

94. Bacri, J.C. and D. Salin, Bistability of ferrofluid magnetic drops under magnetic field. Journal of Magnetism and Magnetic Materials, 1983. 39(1-2): p. 48-50.

95. Bacri, J.C., et al., Phase diagram of an ionic magnetic colloid: Experimental study of the effect of ionic strength. Journal of Colloid and Interface Science, 1989. 132(1): p. 43-53.

96. Bacri, J.C. and D. Salin, Instability of ferrofluid magnetic drops under magnetic field. J Phys (Paris) Lett, 1982. V 43(N 17): p. 649-654.

97. Bacri, J.C., D. Salin, and R. Massart, Study of the deformation of ferrofluid droplets in a magnetic field. Journal de physique. Lettres, 1982. 43(6): p. 179-184.

98. Zeller, C. and W.W. Chen, The instability of a sessile drop of magnetic fluid. Journal of Magnetism and Magnetic Materials, 1983. 39(1-2): p. 165-168.

99. Nguyen, N.T., et al., Magnetowetting and sliding motion of a sessile ferrofluid droplet in the presence of a permanent magnet. Langmuir, 2010. 26(15): p. 12553-12559.

100. Nguyen, N.T., et al., Kinematics and deformation of ferrofluid droplets under magnetic actuation. Microfluidics and Nanofluidics, 2007. 3(5): p. 571-579.

101. Nguyen, N.T., K.M. Ng, and X. Huang, Manipulation of ferrofluid droplets using planar coils. Applied Physics Letters, 2006. 89(5).

102. Lehmann, U., et al., Two-dimensional magnetic manipulation of microdroplets on a chip as a platform for bioanalytical applications. Sensors and Actuators, B: Chemical, 2006. 117(2): p. 457-463.

103. Lehmann, U., et al., Droplet-based DNA purification in a magnetic lab-on-a-chip. Angewandte Chemie - International Edition, 2006. 45(19): p. 3062-3067.

104. Lehmann, U., et al., On-chip antibody handling and colorimetric detection in a magnetic droplet manipulation system. Microelectronic Engineering, 2007. 84(5-8): p. 1669-1672.

105. Bacri, J.C., A.O. Cebers, and R. Perzynski, Behavior of a magnetic fluid microdrop in a rotating magnetic field. Physical Review Letters, 1994. 72(17): p. 2705-2708.

106. Shikida, M., et al., A palmtop-sized rotary-drive-type biochemical analysis system by magnetic bead handling. Journal of Micromechanics and Microengineering, 2008. 18(3).

107. Gijs, M.A.M., F. Lacharme, and U. Lehmann, Microfluidic applications of magnetic particles for biological analysis and catalysis. Chemical reviews, 2010. 110(3): p. 1518-1563.

108. Tondra, M., et al., Design of integrated microfluidic device for sorting magnetic beads in biological assays. Magnetics, IEEE Transactions on, 2001. 37(4): p. 2621-2623.

109. Gijs, M.A.M., Magnetic bead handling on-chip: new opportunities for analytical applications. Microfluidics and Nanofluidics, 2004. 1(1): p. 22-40. 
110. Miltenyi, S., et al., High gradient magnetic cell separation with MACS. Cytometry, 1990. 11(2): p. 231-238.

111. Molday, R., S. Yen, and A. Rembaum, Application of magnetic microspheres in labelling and separation of cells. 1977.

112. Zborowski, M., et al., Continuous-flow magnetic cell sorting using soluble immunomagnetic label. Scientific and Clinical Applications of Magnetic Carriers, 1997: p. 247-258.

113. Zborowski, M., et al., Continuous cell separation using novel magnetic quadrupole flow sorter. Journal of Magnetism and Magnetic Materials, 1999. 194(1): p. 224-230.

114. Zborowski, M., et al., Separations based on magnetophoretic mobility. Separation science and technology, 2002. 37(16): p. 3611-3633.

115. Zborowski, M., et al., Red blood cell magnetophoresis. Biophysical Journal, 2003. 84(4): p. 2638-2645.

116. Moore, L.R., et al., Progenitor cell isolation with a high-capacity quadrupole magnetic flow sorter. Journal of Magnetism and Magnetic Materials, 2001. 225(1): p. 277-284.

117. Nakamura, M., et al., Separation of a breast cancer cell line from human blood using a quadrupole magnetic flow sorter. Biotechnology Progress, 2008. 17(6): p. 11451155.

118. Jing, Y., et al., Blood progenitor cell separation from clinical leukapheresis product by magnetic nanoparticle binding and magnetophoresis. Biotechnology and Bioengineering, 2007. 96(6): p. 1139-1154.

119. Choi, J.-W., et al., A new magnetic bead-based, filterless bio-separator with planar electromagnet surfaces for integrated bio-detection systems. Sensors and Actuators B: Chemical, 2000. 68(1-3): p. 34-39.

120. Watarai, H. and M. Namba, Capillary magnetophoresis of human blood cells and their magnetophoretic trapping in a flow system. Journal of Chromatography A, 2002. 961(1): p. 3-8.

121. Ahn, C.H., et al., A fully integrated micromachined magnetic particle separator. Microelectromechanical Systems, Journal of, 1996. 5(3): p. 151-158.

122. Ahn, C.H. and M.G. Allen, A planar micromachined spiral inductor for integrated magnetic microactuator applications. Journal of Micromechanics and Microengineering, 1999. 3(2): p. 37.

123. Choi, J.W., T.M. Liakopoulos, and C.H. Ahn, An on-chip magnetic bead separator using spiral electromagnets with semi-encapsulated permalloy. Biosensors and Bioelectronics, 2001. 16(6): p. 409-416.

124. Inglis, D.W., et al., Continuous microfluidic immunomagnetic cell separation. Applied physics letters, 2004. 85(21): p. 5093-5095.

125. Deng, T., et al., Manipulation of magnetic microbeads in suspension using micromagnetic systems fabricated with soft lithography. Applied physics letters, 2001. 78(12): p. 1775-1777.

126. Deng, T., M. Prentiss, and G.M. Whitesides, Fabrication of magnetic microfiltration systems using soft lithography. Applied physics letters, 2002. 80(3): p. 461-463. 
127. Hartig, R., et al., Preparative continuous separation of biological particles by means of free-flow magnetophoresis in a free-flow electrophoresis chamber. Electrophoresis, 1992. 13(1): p. 674-676.

128. Hartig, R., M. Hausmann, and C. Cremer, Continuous focusing of biological particles by continuous immuno magnetic sorter: Technique and applications. Electrophoresis, 1995. 16(1): p. 789-792.

129. Fuh, C.B., Peer Reviewed: Split-Flow Thin Fractionation. Analytical chemistry, 2000. 72(7): p. 266-271.

130. Fuh, C.B., H.Y. Tsai, and J.Z. Lai, Development of magnetic split-flow thin fractionation for continuous particle separation. Analytica Chimica Acta, 2003. 497(1-2): p. 115-122.

131. Chronis, N., W. Lam, and L. Lee. A microfabricated bio-magnetic separator based on continuous hydrodynamic parallel flow. in Micro Total Analysis Systems 2001. 2001.

132. Pamme, N., J.C.T. Eijkel, and A. Manz, On-chip free-flow magnetophoresis: Separation and detection of mixtures of magnetic particles in continuous flow. Journal of Magnetism and Magnetic Materials, 2006. 307(2): p. 237-244.

133. Pamme, N. and A. Manz, On-chip free-flow magnetophoresis: continuous flow separation of magnetic particles and agglomerates. Analytical chemistry, 2004. 76(24): p. 7250-7256.

134. Pamme, N. and C. Wilhelm, Continuous sorting of magnetic cells via on-chip freeflow magnetophoresis. Lab on a Chip, 2006. 6(8): p. 974-980.

135. Rong, R., J.W. Choi, and C.H. Ahn, An on-chip magnetic bead separator for biocell sorting. Journal of Micromechanics and Microengineering, 2006. 16(12): p. 2783.

136. Kang, J.H. and J.K. Park, Magnetophoretic Continuous Purification of Single-Walled Carbon Nanotubes from Catalytic Impurities in a Microfluidic Device. Small, 2007. 3(10): p. 1784-1791.

137. Yellen, B.B., O. Hovorka, and G. Friedman, Arranging matter by magnetic nanoparticle assemblers. Proceedings of the National Academy of Sciences of the United States of America, 2005. 102(25): p. 8860-8864.

138. Kose, A.R., et al., Label-free cellular manipulation and sorting via biocompatible ferrofluids. Proceedings of the National Academy of Sciences, 2009. 106(51): p. 21478-21483.

139. Krebs, M.D., et al., Formation of ordered cellular structures in suspension via labelfree negative magnetophoresis. Nano Letters, 2009. 9(5): p. 1812-1817.

140. Zhu, T., F. Marrero, and L. Mao. Continuous separation of non-magnetic particles through negative magnetophoresis inside ferrofluids. in Nano/Micro Engineered and Molecular Systems (NEMS), 2010 5th IEEE International Conference on. 2010: IEEE.

141. Zhu, T., F. Marrero, and L. Mao, Continuous separation of non-magnetic particles inside ferrofluids. Microfluidics and nanofluidics, 2010. 9(4): p. 1003-1009.

142. Gao, Y., et al., Magnetophoresis of nonmagnetic particles in ferrofluids. The Journal of Physical Chemistry C, 2007. 111(29): p. 10785-10791.

143. Zhu, T., et al., Analytical model of microfluidic transport of non-magnetic particles in ferrofluids under the influence of a permanent magnet. Microfluidics and Nanofluidics, 2011. 10(6): p. 1233-1245. 
144. Zhu, T., et al., Continuous-flow ferrohydrodynamic sorting of particles and cells in microfluidic devices. Microfluidics and Nanofluidics, 2012: p. 1-10.

145. Liang, L., J. Zhu, and X. Xuan, Three-dimensional diamagnetic particle deflection in ferrofluid microchannel flows. Biomicrofluidics, 2011. 5(3): p. 034110.

146. Liang, L. and X. Xuan, Diamagnetic particle focusing using ferromicrofluidics with a single magnet. Microfluidics and Nanofluidics, 2012: p. 1-7.

147. Zhu, T., R. Cheng, and L. Mao, Focusing microparticles in a microfluidic channel with ferrofluids. Microfluidics and nanofluidics, 2011. 11(6): p. 695-701.

148. Yellen, B., G. Friedman, and A. Feinerman, Printing superparamagnetic colloidal particle arrays on patterned magnetic film. Journal of Applied Physics, 2003. 93(10): p. 7331-7333.

149. Yellen, B.B. and G. Friedman, Programmable assembly of colloidal particles using magnetic microwell templates. Langmuir, 2004. 20(7): p. 2553-2559.

150. Halverson, D., et al., Manipulation of nonmagnetic nanobeads in dilute ferrofluid. Journal of Applied Physics, 2006. 99(8): p. 08P504-08P504-3.

151. Feinstein, E. and M. Prentiss, Three-dimensional self-assembly of structures using the pressure due to a ferrofluid in a magnetic field gradient. Journal of Applied Physics, 2006. 99(6): p. 064901-064901-6.

152. Friedman, G. and B. Yellen, Magnetic separation, manipulation and assembly of solid phase in fluids. Current opinion in colloid \& interface science, 2005. 10(3): p. 158-166.

153. Yuen, P.K., et al., Microfluidic devices for fluidic circulation and mixing improve hybridization signal intensity on DNA arrays. Lab on a Chip, 2003. 3(1): p. 46-50.

154. Lu, L.H., K.S. Ryu, and C. Liu, A magnetic microstirrer and array for microfluidic mixing. Journal of Microelectromechanical Systems, 2002. 11(5): p. 462-469.

155. Suzuki, H. and C.M. Ho. A magnetic force driven chaotic micro-mixer. in The Fifteenth IEEE International Conference on Micro Electro Mechanical Systems. 2002.

156. Zolgharni, M., et al., A numerical design study of chaotic mixing of magnetic particles in a microfluidic bio-separator. Microfluidics and Nanofluidics, 2007. 3(6): p. 677687.

157. Rong, R., J. Choi, and C. Ahn. A novel magnetic chaotic mixer for in-flow mixing of magnetic beads. in Proc. 7th Int. Conf. on Micro Total Analysis Systems. 2003.

158. Grumann, M., et al., Batch-mode mixing on centrifugal microfluidic platforms. Lab on a Chip, 2005. 5(5): p. 560-565.

159. Mao, L. and H. Koser. Overcoming the diffusion barrier: Ultra-fast micro-scale mixing via ferrofluids. in Solid-State Sensors, Actuators and Microsystems Conference, 2007. TRANSDUCERS 2007. International. 2007.

160. Berthier, J. and F. Ricoul. Numerical modeling of ferrofluid flow instabilities in a capillary tube at the vicinity of a magnet. in International Conference on Modeling and Simulation of Microsystems - MSM. 2002.

161. Wen, C.Y., et al., Rapid magnetic microfluidic mixer utilizing AC electromagnetic field. Electrophoresis, 2009. 30(24): p. 4179-4186.

162. Wen, C.Y., et al., Numerical analysis of a rapid magnetic microfluidic mixer. Electrophoresis, 2011. 32(24): p. 3268-3276. 
163. Blums, E., Some aspects of heat and mass transfer in magnetic fluids. Magnetics, IEEE Transactions on, 1980. 16(2): p. 347-351.

164. Wang, Y., et al., A rapid magnetic particle driven micromixer. Microfluidics and Nanofluidics, 2008. 4(5): p. 375-389.

165. Le, T., Y. Suh, and S. Kang, Efficient Mixing in Microchannel by using Magnetic Nanoparticles. International Journal of Mathematical Models and Methods in Applied Sciences, 2009: p. 58-67.

166. Reza Habibi, M. and M. Ghasemi, Numerical study of magnetic nanoparticles concentration in biofluid (blood) under influence of high gradient magnetic field. Journal of Magnetism and Magnetic Materials, 2011. 323(1): p. 32-38.

167. Furlani, E.P. and X. Xue, Field, force and transport analysis for magnetic particlebased gene delivery. Microfluidics and Nanofluidics, 2012: p. 1-14.

168. Wei, Z.H., C.P. Lee, and M.F. Lai, Magnetic particle separation using controllable magnetic force switches. Journal of Magnetism and Magnetic Materials, 2010. 322(1): p. 19-24.

169. Jafari, A., et al., CFD simulation and evaluation of controllable parameters effect on thermomagnetic convection in ferrofluids using Taguchi technique. Computers \& Fluids, 2008. 37(10): p. 1344-1353.

170. Ryskin, A. and H. Pleiner, Magnetic-field-driven instability in stratified ferrofluids. Physical Review E, 2007. 75(5): p. 056303.

171. Ryskin, A. and H. Pleiner, Influence of a magnetic field on the Soret-effect-dominated thermal convection in ferrofluids. Physical Review E, 2004. 69(4): p. 046301.

172. Hatch, A., et al., A ferrofluidic magnetic micropump. Microelectromechanical Systems, Journal of, 2001. 10(2): p. 215-221.

173. Nguyen, N.T. and M.F. Chai, A stepper micropump for ferrofluid driven microfluidic systems. 2012.

174. Sun, Y., Y.C. Kwok, and N.T. Nguyen, A circular ferrofluid driven microchip for rapid polymerase chain reaction. Lab on a Chip - Miniaturisation for Chemistry and Biology, 2007. 7(8): p. 1012-1017.

175. Sun, Y., N.T. Nguyen, and C.K. Yien, High-throughput polymerase chain reaction in parallel circular loops using magnetic actuation. Analytical Chemistry, 2008. 80(15): p. 6127-6130.

176. Sun, Y., et al., Rapid amplification of genetically modified organisms using a circular ferrofluid-driven PCR microchip. Analytical and Bioanalytical Chemistry, 2009. 394(5): p. 1505-1508.

177. Ohashi, T., et al., A simple device using magnetic transportation for droplet-based PCR. Biomedical Microdevices, 2007. 9(5): p. 695-702.

178. Andò, B., et al. Development of novel ferrofluidic pumps. in Engineering in Medicine and Biology Society, 2006. EMBS'06. 28th Annual International Conference of the IEEE. 2006: IEEE.

179. Andò, B., et al., Ferrofluidic pumps: a valuable implementation without moving parts. Instrumentation and Measurement, IEEE Transactions on, 2009. 58(9): p. 3232-3237.

180. Andò, B., et al., The "One drop" ferrofluidic pump with analog control. Sensors and Actuators A: Physical, 2009. 156(1): p. 251-256. 
181. Hartshorne, H., C.J. Backhouse, and W.E. Lee, Ferrofluid-based microchip pump and valve. Sensors and Actuators B: Chemical, 2004. 99(2): p. 592-600.

182. Hartshorne, H., et al. Development of microfabricated valves for $\mu$ TAS. in Proc. microTAS'98 Workshop. 1998.

183. Yamahata, C., et al., Plastic micropump with ferrofluidic actuation. Microelectromechanical Systems, Journal of, 2005. 14(1): p. 96-102.

184. Ahn, J.J., et al. The viscosity deviation of magnetic fluids for microactuator due to temperature changes. in Microtechnologies in Medicine \& Biology 2nd Annual International IEEE-EMB Special Topic Conference on. 2002: IEEE.

185. Miller, C. and E. Resler, Magnetic forces and the surface instability in ferromagnetic fluids. Physics of Fluids, 1975. 18(9): p. 1112-1118.

186. Greivell, N.E. and B. Hannaford, The design of a ferrofluid magnetic pipette. Biomedical Engineering, IEEE Transactions on, 1997. 44(3): p. 129-135.

187. Ahn, J., J.G. Oh, and B. Choi, A novel type of a microfluidic system using ferrofluids for an application of $\mu$-tas. Microsystem technologies, 2004. 10(8): p. 622-627.

188. Lavrova, O., Numerical methods for axisymmetric equilibrium magnetic-fluid shapes. 2006, Otto-von-Guericke-Universität Magdeburg.

189. Brackbill, J., D.B. Kothe, and C. Zemach, A continuum method for modeling surface tension* 1. Journal of computational physics, 1992. 100(2): p. 335-354.

190. Yap, Y., et al., A global mass correction scheme for the level-set method. Numerical Heat Transfer Part B: Fundamentals, 2006. 50(5): p. 455-472.

191. Liu, J., et al., Numerical and experimental investigations of the formation process of ferrofluid droplets. Microfluidics and Nanofluidics, 2011. 11(2): p. 177-187.

192. Liu, J., Y.F. Yap, and N.-T. Nguyen, Behavior of microdroplets in diffuser/nozzle structures. Microfluidics and Nanofluidics, 2009. 6(6): p. 835-846.

193. Osher, S. and J.A. Sethian, Fronts propagating with curvature-dependent speed: algorithms based on Hamilton-Jacobi formulations. Journal of computational physics, 1988. 79(1): p. 12-49.

194. Peng, D., et al., A PDE-Based Fast Local Level Set Method* 1. Journal of Computational Physics, 1999. 155(2): p. 410-438.

195. Sussman, M., P. Smereka, and S. Osher, A level set method for computing solutions to incompressible two-phase flow. J. Comput. Phys., 1994. 114(1): p. 146-159.

196. Sussman, M., P. Smereka, and S. Osher, A level set approach for computing solutions to incompressible two-phase flow. Journal of computational physics, 1994. 114(1): p. 146-159.

197. Jiang, G.S. and D. Peng, Weighted ENO schemes for Hamilton-Jacobi equations. SIAM Journal of Scientific Computing, 2000. 21(6): p. 2126-2143.

198. Balsara, D.S. and C.W. Shu, Monotonicity Preserving Weighted Essentially Nonoscillatory Schemes with Increasingly High Order of Accuracy. Journal of Computational Physics, 2000. 160(2): p. 405-452.

199. Jiang, G.S. and C.W. Shu, Efficient implementation of weighted ENO schemes. J. Comput. Phys., 1996. 126: p. 202-228.

200. Gottlieb, S. and C.W. Shu, Total variation diminishing Runge-Kutta schemes. Mathematics of Computation, 1998. 67(221): p. 73-85. 
201. Enright, D., et al., A hybrid particle level set method for improved interface capturing. Journal of Computational Physics, 2002. 183(1): p. 83-116.

202. Ho, P.C., et al. A numerical investigation of thermally mediated droplet formation in a T-junction. in Proceedings of the 7th International Conference on Nanochannels, Microchannels, and Minichannels. 2009. Pohang, South Korea.

203. Tan, H., et al., A lab-on-a-chip for detection of nerve agent sarin in blood. Lab on a Chip, 2008. 8(6): p. 885.

204. Hodges, S.R., O.E. Jensen, and J.M. Rallison, Sliding, slipping and rolling: The sedimentation of a viscous drop down a gently inclined plane. Journal of Fluid Mechanics, 2004. 512: p. 95-131.

205. Thurm, S. and S. Odenbach, Magnetic separation of ferrofluids. Journal of Magnetism and Magnetic Materials, 2002. 252: p. 247-249.

206. Wu, Z. and N.T. Nguyen, Convective-diffusive transport in parallel lamination micromixers. Microfluidics and Nanofluidics, 2005. 1(3): p. 208-217.

207. Wu, Z. and N.T. Nguyen, Rapid mixing using two-phase hydraulic focusing in microchannels. Biomedical microdevices, 2005. 7(1): p. 13-20.

208. Odenbach, S. and S. Thurm, Magnetoviscous effects in ferrofluids. Ferrofluids, 2003: p. 185-201.

209. Blums, E., Some new problems of complex thermomagnetic and diffusion-driven convection in magnetic colloids. Journal of Magnetism and Magnetic Materials, 1995. 149(1): p. 111-115.

210. Blums, E., Mass transfer in nonisothermal ferrocolloids under the effect of a magnetic field. Journal of Magnetism and Magnetic Materials, 1999. 201(1): p. 242-247.

211. Patankar, S.V., A calculation procedure for two-dimensional elliptic situations. Numerical Heat Transfer, 1981. 4(4): p. 409-425.

212. Liu, J., Y.F. Yap, and N.T. Nguyen, Numerical study of the formation process of ferrofluid droplets. Physics of Fluids, 2011. 23: p. 072008. 